\title{
Zusammenspiel von Ladungs-, Gitter- und magnetischen Ordnungen in hochdotierten LCMO Schichten
}

\author{
Dissertation \\ zur Erlangung des mathematisch-naturwissenschaftlichen \\ Doktorgrades \\ "Doctor rerum naturalium" \\ der Georg-August-Universität Göttingen \\ im Promotionsprogramm ProPhys \\ der Georg-August University School of Science (GAUSS)
}

vorgelegt von

Florian Fischgrabe

aus Ostercappeln 
Göttingen, 2015

Betreuungsausschuss:

Name, Abteilung/Arbeitsgruppe, Institution

Prof. Dr. Vasily Moshnyaga, I. Physikalisches Institut, Georg-August-Universität Göttingen

Prof. Dr. Martin Dressel, 1. Physikalisches Institut, Universität Stuttgart

Prof. Dr. Konrad Samwer, I. Physikalisches Institut, Georg-August-Universität Göttingen

Mitglieder der Prüfungskommission:

Referent/in

Prof. Dr. Vasily Moshnyaga, I. Physikalisches Institut, Georg-August-Universität Göttingen

Korreferent/in

Prof. Dr. Christian Jooß, Institut für Materialphysik, Georg-August-Universität Göttingen

$\underline{\text { Weitere Mitglieder der Prüfungskommission: }}$

Prof. Dr. Stefan Mathias, I. Physikalisches Institut, Georg-August-Universität Göttingen

Prof. Dr. Michael Seibt, IV. Physikalisches Institut, Georg-August-Universität Göttingen

Prof. Dr. Astrid Pundt, Institut für Materialphysik, Georg-August-Universität Göttingen

Prof. Dr. Hans Hofsäss, II. Physikalisches Institut, Georg-August-Universität Göttingen

Name, Abteilung/Arbeitsgruppe, Institution

Tag der mündlichen Prüfung: 


\section{Inhaltsverzeichnis}

1 Einführung 5

$\begin{array}{lll}2 & \text { Theorie } & 7\end{array}$

$2.1 \quad$ Manganate und ihre relevanten Wechselwirkungen . . . . . . 7

2.1 .1 Hund'sche Regeln . . . . . . . . . . . . . . . . 8

$2.1 .2 \quad$ Kristallfeldaufspaltung . . . . . . . . . . . . . 9

2.1.3 Jahn-Teller-Effekt . . . . . . . . . . . . . . . . 10

2.1.4 Doppelaustausch . . . . . . . . . . . . . 11

2.1 .5 Superaustausch . . . . . . . . . . . . . . . 13

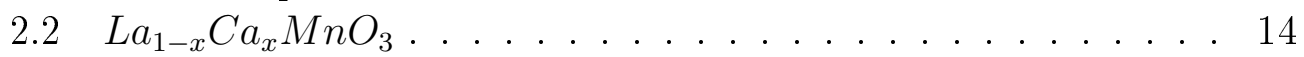

$2.2 .1 \quad$ PM-Phase . . . . . . . . . . . . . . . 15

$2.2 .2 \quad$ CO-Phase . . . . . . . . . . . . . . 15

2.2.3 Modelle für die Ladungsordnung und Korrelation mit realen Strukturen . . . . . . . . . . . . 16

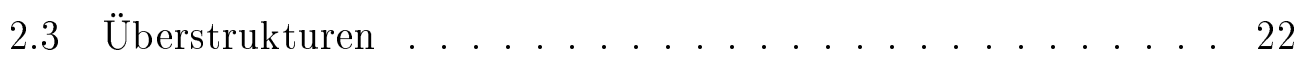

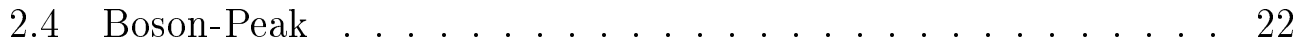

\begin{tabular}{|lll}
\hline 3 & Experimentelle Methoden & 24
\end{tabular}

3.1 Schichtpräparation . . . . . . . . . . . . . . . . . 24

3.2 Strukturelle Untersuchungen . . . . . . . . . . . . . . . . 26

3.2.1 Röntgendiffraktometrie . . . . . . . . . . . 26

3.2 .2 Transmissionselektronenmikroskopie. . . . . . . . . 29

3.2 .3 Rastertunnelmikroskopie . . . . . . . . . . . . 30

3.3 Magnetische Charakterisierung. . . . . . . . . . . . . . . . . . 31

3.4 Transportuntersuchungen . . . . . . . . . . . . . . . . 32

3.5 Optische Spektroskopie . . . . . . . . . . . . . . 33

3.5 .1 Terahertz Spektroskopie . . . . . . . . . . . . . 33

3.5.2 Ferne-Infrarot-Spektroskopie . . . . . . . . . . . . . 33

3.5.3 Raman-Spektroskopie . . . . . . . . . . . . . . 35

3.5.4 Tip enhanced Raman spectroscopy . . . . . . . . . . . 41

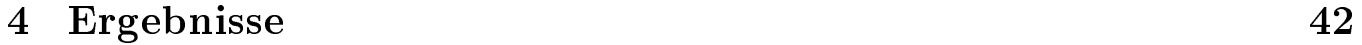

4.1 Evolution der Probenherstellung . . . . . . . . . . . . . . . . 42

4.2 Struktur von LCMO und verwendete Proben . . . . . . . . . . 46

4.3 Röntgendiffraktometrie . . . . . . . . . . . . . . 47

4.3 .1 freistehende Filme . . . . . . . . . . . 55

4.4 Lokale Struktur (TEM) . . . . . . . . . . . . . . . . 61

4.5 Oberflächenmorphologie $(\mathrm{STM}) \ldots \ldots$. . . . . . . . . 66

4.6 Bestimmung der Schichtdicke durch SEM . . . . . . . . . . . 67 
4.7 Transportmessungen mit dem PPMS . . . . . . . . . . . 68

4.8 Magnetische Charakteristika . . . . . . . . . . . . . . 70

4.9 Optische Eigenschaften . . . . . . . . . . . . . . . 77 4.9 .1 THz-Spektroskopie . . . . . . . . . . . . . . 77

4.9 .2 FIR-Spektroskopie . . . . . . . . . . . . . . . . . . 79

4.9 .3 Ramanspektroskopie . . . . . . . . . . . . . 86

$4.9 .4 \quad$ TERS $\ldots \ldots \ldots \ldots \ldots . \ldots \ldots$

5 Zusammenfassung und Diskussion 110

5.1 Probenqualität . . . . . . . . . . . . . . . . . 110

$5.1 .1 \quad$ Filme auf $\mathrm{MgO}$. . . . . . . . . . . . . . . . . 110

5.1 .2 freistehende Filme . . . . . . . . . . . . . 113

5.2 Ladungsordnung . . . . . . . . . . . . . . . . . . . 114

5.3 Zusammenhang der verschiedenen Ordnungs- und Unordnungs-

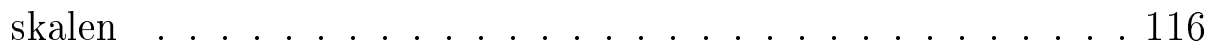

\begin{tabular}{llr}
\hline 6 & Ausblick & 119
\end{tabular}

\begin{tabular}{lll}
\hline 7 & Literatur & 121
\end{tabular}

$\begin{array}{lll}8 & \text { Appendix } & 131\end{array}$

9 Danksagung 159 


\section{Einführung}

Um alle Effekte, die in Festkörpern eine Rolle spielen können, aufzuzählen, bedürfte es schon einiger Zeit. Das Verständnis auf welche Art und Weise unterschiedliche Wechselwirkungen sich gegenseitig beeinflussen ist sogar so komplex, dass es größtenteils noch nicht verstanden ist. Die Manganate stellen in dieser Hinsicht ein prototypisches System von Verbindungen dar, deren interne Wechselwirkungen alle eine ähnliche Größenordnung aufweisen, die durch Dotierungen stark beeinflusst werden kann. Dadurch werden sie zu geeigneten Studienobjekten, wenn es um die gegenseitige Beeinflussung unterschiedlichster elementarer Wechselwirkungen geht. In den Manganaten lassen sich unterschiedlichste kolossale Effekte beobachten, die auf dem plötzlichen Kollaps eines Zustands aufgrund von angelegten Feldern beruhen. So ist es nicht verwunderlich, dass die Manganate besondere Aufmerksamkeit erhielten als 1993 R. von Helmolt et. al. den CMR-Effekt entdeckten [1]. Durch ein starkes Magnetfeld, $\mathrm{B}=7 \mathrm{~T}$, das an die Manganate, die einen Metallisolatorübergang (MIT) zeigen, angelegt wird, können, bei Temperaturen in der Nähe des Metallisolatorübergangs, Widerstandsänderungen von bis zu $10^{10 \%}$ erzielt werden [2 $[4]$. In späteren Arbeiten zu dem Thema wurde dann immer klarer, dass der CMR-Effekt auf einem perkolativen Übergang beruht und die zweite Phase, die eminent mit dem CMR-Effekt verbunden ist, eine ladungsgeordnete antiferromagnetische Phase ist [5]-8]. Das Zusammenspiel der beiden Phasen und ihr perkolativ getriebener Ubergang sind daher von größter Bedeutung, um eine mögliche kommerzielle Nutzung als magnetischer RAM oder für Festplatten zu ermöglichen. Die hier vorgelegte Arbeit konzentriert sich auf die zweite dieser beiden Phasen, die ladungsgeordnete, antiferromagnetische Phase. Diese wird am überdotierten System $\mathrm{La}_{1-x} \mathrm{Ca}_{x} \mathrm{MnO}_{3}$ (LCMO) untersucht. Trotz vieler Arbeiten zum Thema gibt es vergleichsweise wenige Untersuchungen, die sich mit der ladungsgeordneten Phase beschäftigen, obwohl schon frühe Experimente suggerierten, dass die Perkolation des Systems eine wichtige Rolle spielt [6, 9]. Deshalb ist das Ziel dieser Arbeit die ladungsgeordnete Phase weiter zu untersuchen und gleichzeitig eine Weiterführung vorhergegangener Experimente an Polykristallen $[10 \mid$ zu vollziehen, die ein tiefer gehendes Verständnis der Ladungsordnung in LCMO ermöglichen soll. Die Experimente an polykristallinen LCMO Keramiken haben dabei einen Bosonpeak zum Vorschein gebracht, der ein Indiz für Unordnung im System darstellt. Zur Untersuchung ob auch die ladungsgeordnete Phase einen perkolativen Übergang zeigt und der Bosonpeak auch in einkristallinen Proben vorhanden ist, wurden mit der Metallorganischen-Aerosol-Deposition LCMO Schichten hergestellt und nach eingehender Charakterisierung für optische Messungen in Stuttgart zur 
Verfügung gestellt. Im Rahmen des DFG Projekts DR 228/36 wurden von Boris Gorshunov und Elena Zhukova THz- und FIR-Messungen an den hergestellten Schichten durchgeführt, deren Ergebnisse in dieser Arbeit ebenfalls gezeigt werden. Besonderes Augenmerk wurde hierbei auch auf die in der Literatur viel diskutierte Phasenseparation gelegt [5, 6, 11]. Es gibt auch für die hochdotierten LCMO Proben Arbeiten die Phasenseparation in Nanokristallite zeigen [8, 9, 12, Die Grenzflächenphase ist hierbei eine ferromagnetisch metallische Phase. Das Verhalten der überdotierten Phase ist damit dem Verhalten im Bereich des CMR-Effekts ähnlich. Weitere Kenntnis über den ladungsgeordneten Zustand könnte somit Möglichkeiten eröffnen die Magnetfelder, die typischerweise für einen großen Magnetwiderstand der Manganate benötigt werden, deutlich zu reduzieren und somit die Manganate für Sensorik oder Speicherelemente nutzbar zu machen. Es gibt allerdings unterschiedliche ladungsgeordnete Phasen in den Manganaten, die stark von den verwendeten Materialien abhängen. Daher ist es wichtig für jedes Material, das als Sensor oder Speicherelement verwendet werden soll, auch den ladungsgeordneten Zustand zu untersuchen, wenn das vorhandene Material Zeichen von Phasenseparation zeigt. 


\section{Theorie}

\subsection{Manganate und ihre relevanten Wechselwirkungen}

Die Manganate stellen eine Materialklasse von zumeist kristallinen neutralen Verbindungen dar. Die allgemeine Summenformel lautet $\mathrm{AMnO}_{3}$. Die A-Plätze werden hierbei typischerweise durch Elemente aus der Gruppe der Lanthanoide besetzt während die BPlätze durch Manganionen okkupiert werden.

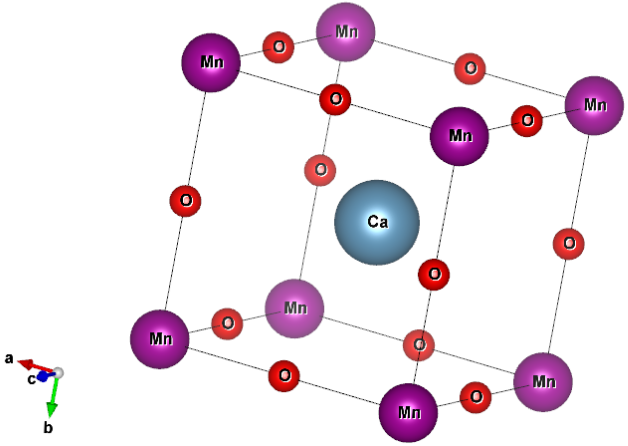
Die Struktur besteht aus vier Manganatomen die von einem Oktaeder Sau-

Abbildung 1 - Die Einheitszelle des $\mathrm{CaMnO}_{3}$ Prototypen in der pseudokubischen Repräsentation erstoffatome umgeben sind.

Hierbei teilen sich zwei Manganatome ein Sauerstoffatom. Innerhalb des kubischen Gitters, das durch die Manganatome aufgespannt wird, befindet sich nun das A-Atom. Die Manganate treten typischerweise in zwei verschiedenen Kristallstrukturen auf, der orthorhombischen Pnma Struktur und der rhomboedrischen $R \overline{3} c$ Struktur, können aber auch in der monoklinen Struktur auftreten. Die Struktur wird hierbei durch viele Parameter bestimmt. Eine Änderung der Kristallstruktur bei unterschiedlicher Dotierung tritt typischerweise durch eine Änderung des Toleranzfaktors $t=\frac{r_{A}+r_{O}}{\sqrt{2}\left(r_{B}+r_{O}\right)}$ auf. Hierbei ist $r_{A}$ der Radius des Atoms auf dem Gitterplatz A, $r_{B}$ der Radius des Atoms auf dem Gitterplatz B und $r_{O}$ der Radius des Sauerstoffatoms. Dies ist allerdings nicht die einzige Möglichkeit eine Änderung der Kristallstruktur zu bewirken. So zeigen Experimente, dass eine Änderung der Kristallstruktur durch eine Änderung der Temperatur, des magnetischen Feldes, des elektrischen Feldes und sogar durch Laserillumination bewirkt werden kann [2, 8, 13, 14. Außerdem können Materialien die prinzipiell denselben Toleranzfaktor haben wie zum Beispiel $L a_{0.70} \mathrm{Ca}_{0.11} \mathrm{Sr}_{0.19} \mathrm{MnO}_{3}$ und $\mathrm{La}_{0.32} \mathrm{Pr}_{0.38} \mathrm{Sr}_{0.30} \mathrm{MnO}_{3}$ unterschiedliche Kristallstrukturen aufweisen [15, 16]. Dies führt zu der Erkenntnis, dass das einfache Prinzip des Toleranzfaktors viel zu kurz gegriffen ist für die Manganate. Der Grund ist die große Vielfalt an attraktiven und repulsiven Wechselwirkungen, die zum größten Teil alle in derselben Größen- 
ordnung liegen und somit eine einfache Beschreibung durch simple Prinzipien wie den Toleranzfaktor ad absurdum führen. Daher soll nun im Folgenden versucht werden jede der wechselwirkenden Kräfte zu beschreiben und in ein größeres Gesamtbild für die Manganate einzuordnen, hierbei sollen die Wechselwirkungen nach ihrer Größe absteigend sortiert werden.

\subsubsection{Hund'sche Regeln}

Die Wechselwirkung in Manganaten mit dem größten Energiebetrag ist die Hund'sche Kopplungsenergie. Soviel Energie ist nötig wenn man zwei Elektronen unterschiedlichen Spins in das gleiche Orbital zwingen möchte. Aufgrund der Größe der Hund'schen Kopplungsenergie werden die meisten Materialien nach drei einfachen Regeln mit Elektronen aufgefüllt. Die sogenannten Hund'schen Regeln:

Regel 1:

Maximierung des Spins: Die zur Verfügung stehenden Orbitale werden so aufgefüllt, dass der Spin des Gesamtsystems maximal ist. Das bedeutet möglichst viele Spins müssen parallel stehen.

Regel 2:

Maximierung des Gesamt-Bahndrehimpulses: Nachdem Regel 1 erfüllt wurde wird weiterhin so aufgefüllt, dass der Bahndrehimpuls $\mathrm{L}=|\mathrm{m}|+(|\mathrm{m}|-1)$ maximiert wird.

Regel 3:

Maximierung des Gesamtdrehimpulses: Nachdem Regel 1 und 2 erfüllt wurden soll nun auch der Gesamtdrehimpuls $\mathrm{J}=\mathrm{L}+\mathrm{S}$ maximiert werden.

Möchte man hingegen die Regel 1 missachten und ein Elektron in ein bereits besetztes Orbital setzen mit entgegengesetztem Spin ist dafür eine Energie von typischerweise $2-3 \mathrm{eV}$ nötig. Da es keinen Energiebeitrag in den Manganaten gibt der groß genug wäre um diese Hund'sche Kopplungsenergie zu überwinden werden die Manganate immer nach diesen Regeln aufgefüllt. Dadurch können keine low-spin Komplexe entstehen wie sie in manchen anderen Oxiden vorkommen. 


\subsubsection{Kristallfeldaufspaltung}

Der zweitgrößte Energiebeitrag wird von der Kristallfeldaufspaltung geliefert, von typischerweise $\Delta_{C F}=1-2 e V$ in Manganaten. Das Prinzip der Kristallfeldaufspaltung stammt aus der Ligandentheorie der Chemie. Dahinter steckt ein eigentlich recht einfaches Prinzip. Die Manganatome im kubischen Gitter haben als äußerste Elektronen 3d-Zu-

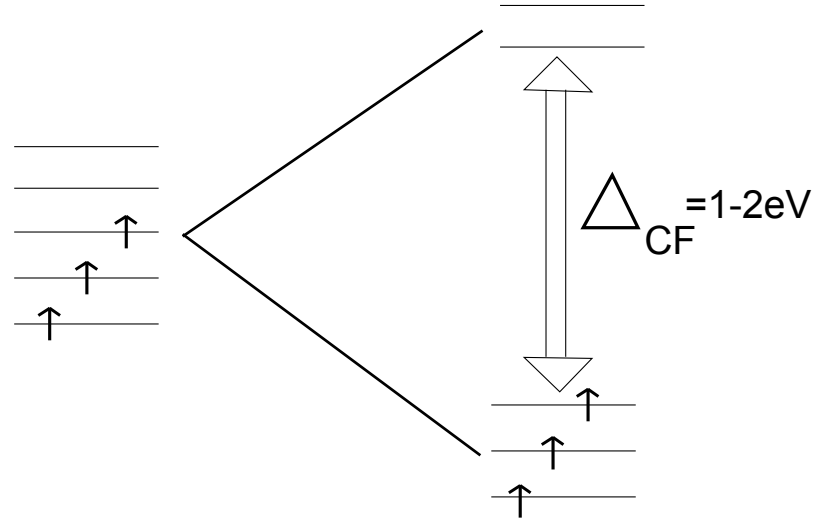

Abbildung 2 - Die Aufspaltung der d-Orbitale aufgrund des Kristallfelds in die untenliegenden $t_{2 g^{-}}$und die obenliegenden $e_{g}$-Orbitale stände. Diese elektronischen Zustände sind verglichen mit s-Wellen sehr viel stärker gerichtet und lokalisiert. Die fünf möglichen d-Wellen Zustände sind entweder entlang der Kristallachsen oder entlang der Raumdiagonalen ausgerichtet. Betrachtet man nun die fünf Zustände erkennt man sofort, dass in einer kubischen Umgebung, in der die Sauerstoffatome entlang der Achsen des Koordinatensystems liegen, einige der Zustände energetisch ungünstiger sind als die anderen. Dies ist der alleinigen Tatsache geschuldet, dass die Elektronen sich den Platz mit den Elektronen der Sauerstoffatome entlang der Achsen teilen müssen und dadurch eine erhöhte Coulombabstoßung erfahren, die effektiv diese Energieniveaus anhebt. Hierdurch ergeben sich anstatt ehemals einem nun zwei energetisch entartete Zustände. Die Zustände höherer Energie, genannt $e_{g}$-Zustände, sind dabei zweifach entartet während die Zustände niedrigerer Energie, genannt $t_{2 g}$, dreifach entartet sind. Das Energieschema der neuen Zustände im Vergleich zu den ursprünglichen d-Zuständen ist in Graph 2 zu sehen. Die Manganionen treten hierbei in den Oxidationsstufen $3+$ und $4+$ auf. Da die Energie des Kristallfelds geringer ist als die Hund'sche Kopplungsenergie wird je nach Oxidationsstufe ein $e_{g}$-Orbital besetzt oder nicht besetzt, da erst alle Orbitale mit einer Spinrichtung aufgefüllt werden müssen. Der Effekt ist für die Manganate von großer Wichtigkeit, da die Tatsache ob das $e_{g}$-Orbital besetzt wird oder nicht, sowohl für den Doppelaustausch als auch für den Jahn-Teller-Effekt eine große Rolle spielt. 


\subsubsection{Jahn-Teller-Effekt}

Der Jahn-Teller-Effekt stammt direkt aus dem Jahn-Teller Theorem von 1937 [17. Dieses Theorem besagt, dass entartete Zustände instabil sind wenn sie nicht auf der Zeitumkehrinvarianz beruhen. Der doppelt entartete $e_{g}-\mathrm{Zu}-$ stand des $\mathrm{Mn}^{3+}$ Ions ist ein solcher Zustand. Von vornherein ist nicht festgelegt, welches der beiden Orbitale $3 z^{2}-$ $r^{2}$ oder $x^{2}-y^{2}$ besetzt wird. Diese orbitale Entartung wird nun durch das System selbst verhindert, indem sich ei-

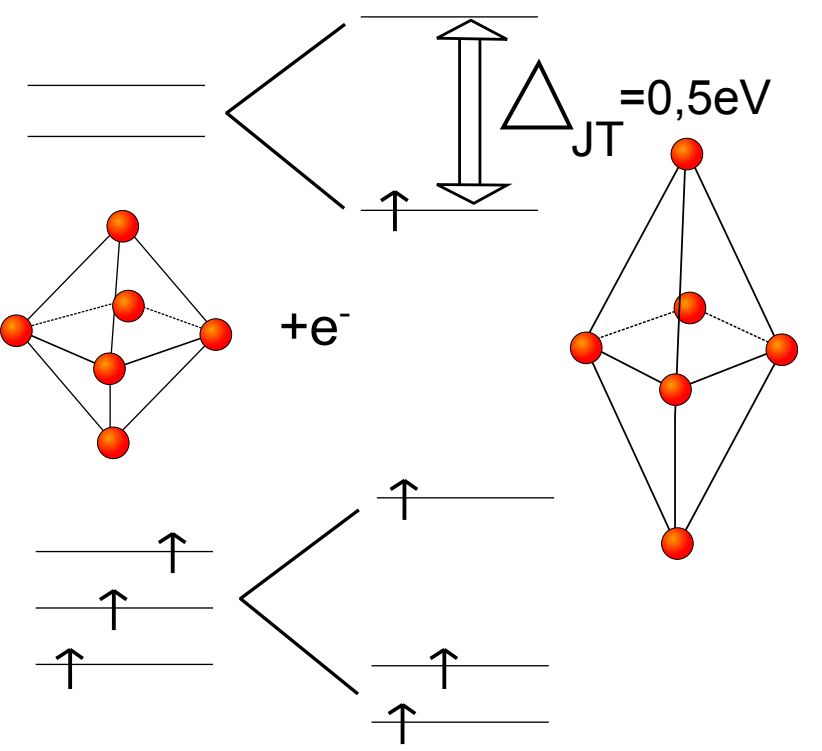

Abbildung 3 - Energieschema des Jahn-TellerEffekts sowie eine deutlich überzeichnete Streckung des Oktaeders entlang des $z^{2}$-Orbitals ne Verzerrung des Oktaeders bildet. Dieses Phänomen ist bekannt als Jahn-Teller-Effekt. Es sorgt im prototypischen Material $\mathrm{LaMnO}_{3}$ für eine antiferromagnetische (AFM) Ordnung durch einen Effekt genannt: kooperativer Jahn-Teller-Effekt. Dieser Effekt existiert allerdings nur für den Prototypen und für sehr kleine Dotierungen mit Strontium [18]. Nichtsdestotrotz ist die Energieskala des Jahn-Teller-Effekts typischerweise $\Delta_{J T}=0,5 \mathrm{eV}$, wodurch es einen großen Unterschied macht ob ein $\mathrm{Mn}^{3+}$ oder $\mathrm{Mn}^{4+}$ Ion vorliegt. Liegt ein $\mathrm{Mn}^{3+}$ Ion vor wird typischerweise eine der Achsen des Oktaeders verlängert, während die anderen beiden gestaucht werden. 


\subsubsection{Doppelaustausch}

Der Doppelaustausch ist eine Austauschwechselwirkung, die 1951 zuerst von Zener vorgeschlagen wurde [19]. Der Doppelaustausch verbindet elektrische Leitfähigkeit und magnetische Ordnung. Betrachten wir die Manganionen im Kristallgitter, so sind diese entweder dreifach positiv, $d^{4}$ Konfiguration, oder vierfach positiv, $d^{3}$ Konfiguration, abhängig von der Dotierung. Die Elektronen liegen in der Highspin Konfiguration vor, was bedeutet, dass alle Elektronenspins in dieselbe Richtung zeigen, aufgrund der oben erwähnten Hund'schen Kopplungsenergie. Diese Tatsache sorgt nun für

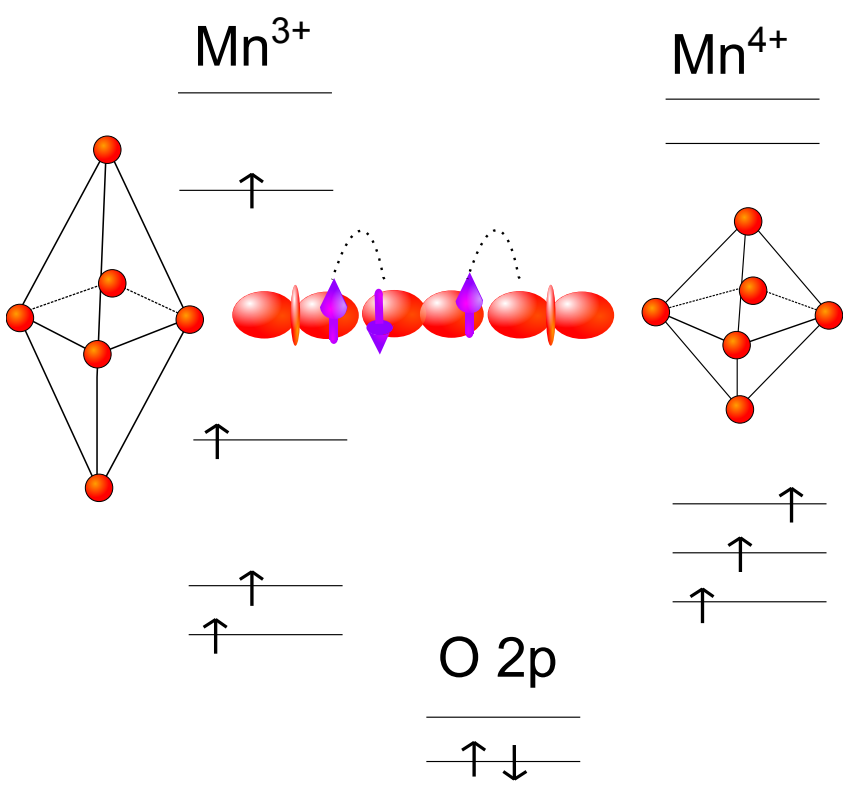

Abbildung 4 - Energieschema des Doppelaustauschs unter Berücksichtigung der verzerrten und unverzerrten Struktur des Oktaeders. Der Austausch wird über die p-Orbitale des Sauerstoffs vermittelt, die energetisch tiefer liegen als die d-Orbitale. einen interessanten Effekt.

Die Manganionen teilen sich den Sauerstoff an jeder Ecke des Oktaeders immer mit einem weiteren Manganion. Entlang dieses Pfades kann nun ein Austausch stattfinden. Ein $\mathrm{Mn}^{3+}$ das sich ein Sauerstoff mit einem $\mathrm{Mn}^{4+}$ teilt ist eine starre und unbewegliche Konfiguration. Was passiert aber nun, wenn die beiden Ionen abwechselnd das weitere Elektron aufnehmen? Genau diese Frage hat sich damals Zener gestellt und kam zu einer recht bemerkenswerten Antwort. Da das Elektron nun weiter verteilt ist, ist somit auch seine Energie geringer. Die verteilte Aufenthaltswahrscheinlichkeit des Elektrons ist damit energetisch günstiger. Der Austausch funktioniert wie folgt: Das Elektron, das im $e_{g}$-Orbital des $M n^{3+}$ sitzt tunnelt mit einer Tunnelwahrscheinlichkeit t in das $e_{g}$-Orbital des $\mathrm{Mn}^{4+}$. Dieser Austausch wird über den Sauerstoff mediiert und hängt essenziell vom Bindungswinkel der Manganatome zum Sauerstoff ab. Je näher der Winkel an $180^{\circ}$ liegt, desto größer die Tunnelwahrscheinlichkeit. Wichtiger Teilaspekt des ganzen Tunnelprozesses ist, dass ein Tunneln nur dann energetisch sinnvoll ist, wenn die Spins der 
beiden Manganionen in dieselbe Richtung zeigen, da ansonsten beim Tunnelvorgang die Hundsche Kopplungsenergie aufgebracht werden müsste, um den Elektronenspin zu drehen. Dies sorgt dafür, dass sich die Manganate bei Dotierung mit Elementen aus der zweiten Hauptgruppe, die also $M n^{4+}$ Ionen erzeugen, unterhalb einer gewissen Temperatur als Ferromagnete präsentieren. Die Formel für die Doppelaustauschenergie lässt sich nach Hasegawa und Anderson wie folgt angeben [20]:

$$
H_{D E}=-\sum_{i, j}\left(t \cdot \cos \left(\frac{\Theta_{i, j}}{2}\right) c_{i}^{+} c_{j}+J_{H} \sum_{i}\left(S_{i} S_{j}\right)\right.
$$

Hierbei ist $\Theta_{i, j}$ der Winkel zwischen den Spins der beiden Manganionen, $c_{i}^{+} c_{j}$ die Erzeugungs- und Vernichtungsoperatoren und $S_{i} S_{j}$ die Spins der Manganionen. Besonders prominent ist hier die Vereinfachung zu einem Kosinus, der in ihrer Arbeit als Erstes auftauchte. Das Tunnelelement t spielt neben den Winkeln der Spins die wichtigste Rolle und soll nun noch etwas näher betrachtet werden. Für die Größe des Tunnelelements sind verschiedene Größen entscheidend, unter anderem der oben bereits erwähnte Winkel der Mn-O-Mn Bindung. Der Grund ist hier darin zu suchen, dass die Tunnelwahrscheinlichkeit exponentiell mit der Barrierenhöhe ansteigt. Die Barrierenhöhe ist über den Grad der Hybridisierung der p-Orbitale des Sauerstoffs mit den d-Orbitalen des Mangan verbunden. Diese Hybridisierung auf deren Wichtigkeit Goodenough hingewiesen hat, ist stark verknüpft mit dem oben genannten Winkel. Je weiter der Winkel von den idealen $180^{\circ}$ abweicht, desto geringer die Hybridisierung und desto geringer somit die Tunnelwahrscheinlichkeit. Dies schlägt sich dann sowohl im elektrischen Widerstand, als auch in den ferromagnetischen (FM) Eigenschaften nieder. Interessanterweise kann das Tunnelelement mit so etwas trivialem wie dem Toleranzfaktor in Verbindung gebracht werden. Je näher der Toleranzfaktor an eins, desto näher sind die Bindungswinkel Mn-O-Mn an $180^{\circ}$ und desto besser sind Leitfähigkeit und magnetische Eigenschaften. Dies zeigt sich am Beispiel von LCMO und LSMO. Obwohl von der Elektronenkonfiguration gleich, bilden die beiden Materialien, auf Grund der Größe der Ionen, Manganate mit unterschiedlichem Toleranzfaktor und zeigen deutlich unterschiedliches Verhalten für den Widerstand, als auch Magnetisierung. Wo LCMO einen Metallisolatorübergang mit großem CMR-Effekt zeigt, ist der Effekt bei LSMO sehr viel kleiner und bei höheren Temperaturen. Da der Toleranzfaktor von LSMO näher an 1 liegt als der von LCMO, ist es für das Material leichter metallisch und magnetisch zu bleiben. LCMO hingegen ist sehr empfindlich auf Magnetfelder, da der Toleranzfaktor weiter von eins abweicht. Dies sorgt für den deutlich größeren CMR-Effekt [16]. Die Energien, die für den Doppelaustausch typischerweise angegeben werden, liegen bei 0,1-0,3 eV [21,22]. 


\subsubsection{Superaustausch}

Es gibt eine weitere Austauschwechselwirkung die in Manganaten $\mathrm{zu}$ finden ist, der sogenannte Superaustausch. Hierbei handelt es sich um ein Prinzip das damals 1950 von Anderson entdeckt wurde 23. Wenn sich magnetische Ionen in einem Gitter anordnen, entstehen zwischen ihnen Austauschwechselwirkungen, die von dem Abstand der Ionen und der Orbitalkonfiguration abhängen. Sind die Orbitale gerichtete
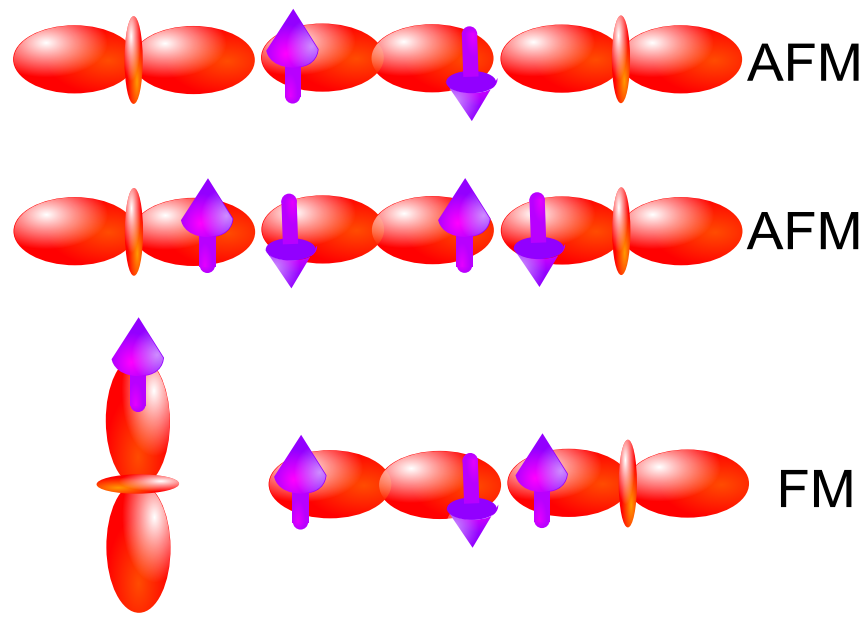

Abbildung 5-Der Superaustausch bewirkt je nach Füllung der Orbitale eine ferro- oder antiferromagnetische Ausrichtung

d-Orbitale gibt es eine ge-

genseitige Wechselwirkung. Diese Wechselwirkung wird Superaustausch genannt und über virtuelle Sprungprozesse über die Sauerstoffatome, die entlang der Achsen liegen vermittelt, wodurch die Hybridisierung mit den Sauerstoffen einen großen Einfluss auf die Stärke dieser Wechselwirkung hat. Hierbei geht es im Einzelnen wieder um die, dieses mal virtuellen, Tunnelwahrscheinlichkeiten der Elektronen von Ion zu Ion. Diese virtuellen Austauschprozesse wurden zuerst von Anderson beschrieben. Er zeigt darin, dass zwei Manganatome, die in einer Richtung durch ein Sauerstoffatom verbunden sind ihren Spin antiferromagnetisch ausrichten, um einen virtuellen Austausch der Manganelektronen zu ermöglichen. Wenn die Rumpfspins der $t_{2 g^{-}}$ Orbitale antiferromagnetisch koppeln, gibt es eine Tunnelwahrscheinlichkeit von den Sauerstoffen auf die jeweiligen benachbarten Manganionen. Diese Tunnelwahrscheinlichkeit senkt die kinetische Energie der Elektronen ab und sorgt damit für einen Energiegewinn bei antiferromagnetischer Ausrichtung. Die Wechselwirkung ändert sich je nach Geometrie der Verbindung. Verbindungen die einen $90^{\circ}$ Winkel zueinander aufweisen zeigen eine leicht ferromagnetische Wechselwirkung. Mit diesen Goodenough-Kanamori-Anderson Regeln ließ sich erstmals erklären, warum $\mathrm{LaMnO}_{3}$ einen antiferromagnetischen Grundzustand bildet |9|. Die Superaustauschenergie wird in unterschiedlichen Arbeiten ebenfalls mit Energien zwischen 0,1-0,3 eV angegeben [24,25]. 


\section{$2.2 \quad L a_{1-x} C a_{x} \mathrm{MnO}_{3}$}

Das in dieser Arbeit untersuchte System ist Lanthancalciummanganat $\mathrm{La}_{1-x} \mathrm{Ca}_{x} \mathrm{MnO}_{3}$ (LCMO) im Dotierungsbereich $0,5 \leq x \leq 1$. Der hohe Dotierungsbereich größer als 0,5 ist ein Bereich, dem in vielen Studien wenig Aufmerksamkeit geschenkt wird. Die meisten Studien konzentrieren sich auf den Dotierungsbereich 0,2-0,45 in dem LCMO den CMR-Effekt zeigt. Was in vielen Arbeiten wenig Beachtung findet, ist die Tatsache, dass sich der Bereich des Phasendiagramms in dem der CMR-Effekt auftritt nur durch ein ebenso gutes Verständnis des gesamten Phasendiagramms verstehen lässt. Der CMR-Effekt entsteht so zum Beispiel aus dem Zusammenspiel von ladungsgeordneter isolierender Phase und der ferromagnetisch metallischen Phase [5, 7, 26]. Durch diese Tatsache inspiriert sollte man vermuten, dass dem ladungsgeordnetem Bereich eine hohe Aufmerksamkeit zuteilwird, was allerdings nicht der Fall ist. Im Gegenteil ist die grundlegende Natur des ladungsgeordneten Zustands zwar theoretisch charakterisiert aber nicht eingehend verstanden. So ist zum Beispiel immer noch unklar wie stark der Einfluss von orbitaler Ordnung in der ladungsgeordneten Phase ist und welche Verbindung mit den magnetischen Eigenschaften besteht [2, 27]. Außerdem unklar bleibt ob die Ladungsordnung der energetisch günstigste Zustand in Einkristallen ist, da diese nicht synthetisiert werden können.

In Abbildung 6 ist ein Phasendiagramm aus der Arbeit von Pissas und Kallias des Dotierungsbereichs von $=0,48-1$ zu sehen [28]. Das Phasendiagramm weist mehrere Phasen auf, die sowohl von der Temperatur, als auch von der Dotierung abhängen. Besonders erwähnenswert sind hierbei die ladungsgeordnete Phase im Bereich von $0,5 \leq x \leq 0,85$, die monokline Phase im Bereich von $0,79 \leq x \leq 0,87$ und die antiferromagnetische Phase die von $\mathrm{x}=0,5$ bis $\mathrm{x}=0,9$ reicht. Die Hochtemperaturphase von LCMO ist hierbei ein paramagnetisches Metall, dessen Ausprägung oft durch Korngrenzen und Störstellen in den Proben gestört wird, wodurch diese isolierend wirken. Dies ist allerdings der alleinigen Tatsache geschuldet, dass der Widerstand der metallischen Phase im Bereich von Raumtemperatur extrem gering ist, mit typischerweise $10^{-4} \Omega \mathrm{cm}$ und sie dadurch erst bei höheren Temperaturen sichtbar wird. Es ist bisher nicht gelungen, mit Ausnahme von extrem hochdotiertem LCMO $x \geq 0,9$, Einkristalle von n-dotiertem LCMO zu wachsen. Der Grund dafür wird oft in der Volatilität des Mangans vermutet [29]. Damit sind einkristalline Schichten die reinsten Kristalle, die sich für wissenschaftliche Untersuchungszwecke herstellen lassen. Des Weiteren gibt es viele Verfahren zur Synthese von Keramikproben und Polykristallen 29,30. 


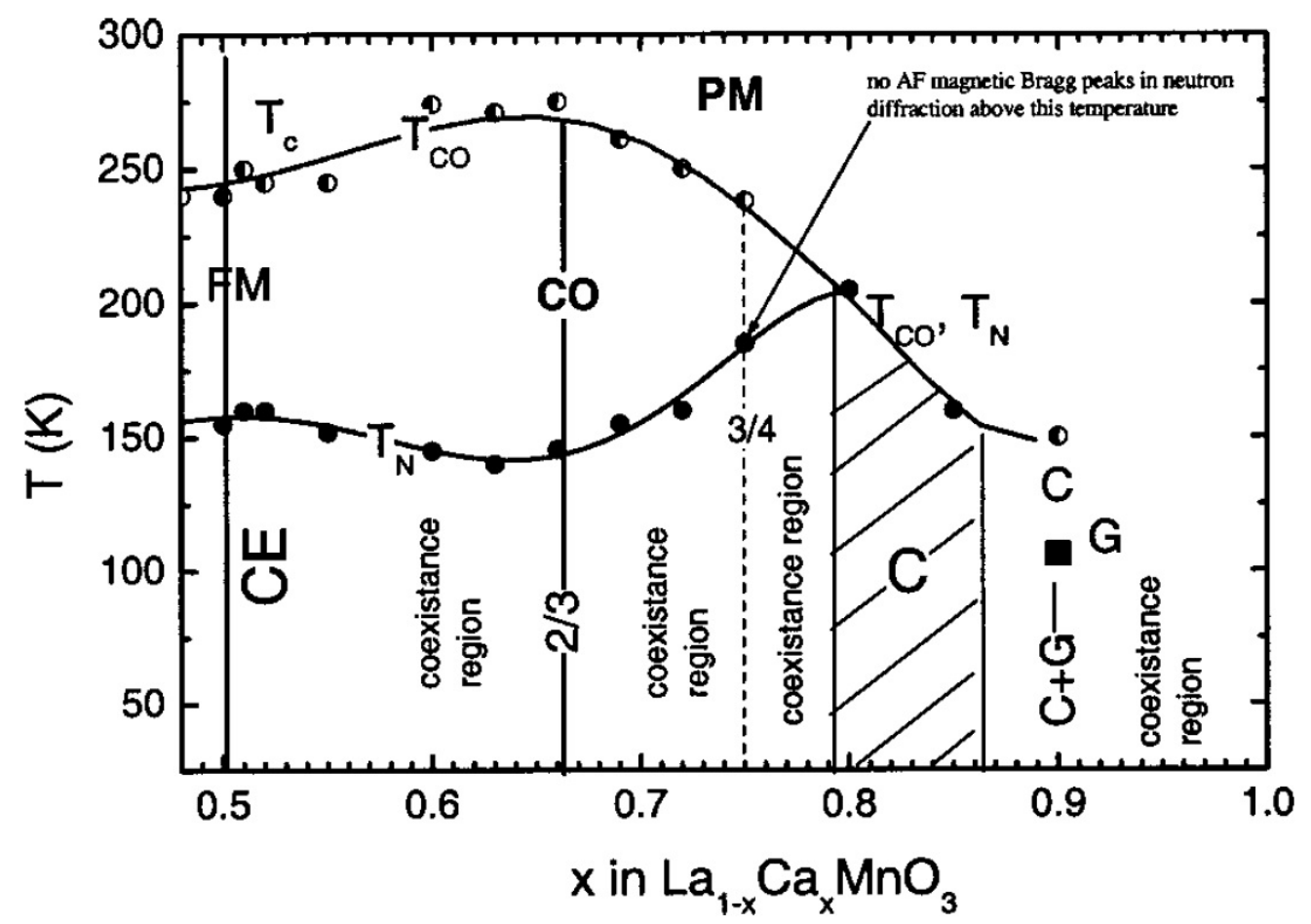

Abbildung 6 - Das Phasendiagramm von LCMO für Dotierungen $x>0,48$ aus der Arbeit von Pissas und Kallias [28]

\subsubsection{PM-Phase}

Wie in Abbildung 6 zu erkennen ist, zeigt hochdotiertes LCMO im gesamten Dotierungsbereich von $0,5 \leq x \leq 1$ ein paramagnetisches metallisches Verhalten bei Temperaturen oberhalb von $275 \mathrm{~K}$, die sogenannte PM-Phase. Der Grund hierfür ist die entartete Struktur des elektronisch dotierten Halbleiters. Sehr reines LCMO sollte daher bei sehr hohen Temperaturen immer einen metallischen Anstieg des Widerstands mit der Temperatur zeigen, wobei sich der Punkt, ab dem metallisches Verhalten beobachtet werden kann in Abhängigkeit von x verschiebt 31,32. Die PM-Phase zeigt im Dotierungsbereich $0,5 \leq x \leq 0,85$ einen Phasenübergang zu einer ladungsgeordneten Phase und im Dotierungsbereich $x>0,85$ einen direkten Übergang in einen verkanteten Antiferromagneten.

\subsubsection{CO-Phase}

Im Dotierungsbereich von $0,5 \leq x \leq 0,85$ zeigt LCMO unterhalb einer Temperatur $T_{C O}$ genannt Ladungsordnung $(\mathrm{CO})$. Hierbei gibt es drei stabile und eine metastabile Phase. Die stabilen Phasen sind $x=0,5, x=0,66$ und 
$\mathrm{x}=0,85$, die metastabile Phase ist $x=0,75[9,28]$. Dotierungen zwischen diesen stabilen Phasen werden durch das Hebelgesetz realisiert. Die Ladung liegt hierbei auf den Manganatomen, die jeweils als $M n^{3^{+}+\delta}$ oder $M n^{4^{+}-\delta}$ vorkommen. Diese Ladungen ordnen im Kristallgitter nun auf verschiedene Art und Weise. Für die $x=0,5$ Phase hat jedes $M n^{3^{+}+\delta}$ als Nachbarn ein $M n^{4^{+}-\delta}$, damit wird genau die halbe Füllung realisiert und die Ladungsordnung kann, zum Beispiel mit Elektronenbeugung, als Übergitterpeaks sichtbar gemacht werden. Die $x=0,66$ Phase zeigt eine streifenartige Ladungsordnung. Die unterschiedlichen Valenzen der Manganionen zeigen sich hier in Streifen entlang der [100] Richtung, wobei zwei Lagen $M n^{4^{+}-\delta}$ von einer Lage $M n^{3^{+}+\delta}$ abgeschlossen werden. Die metastabile Phase von $x=0,75$ kommt dadurch zustande, dass man eine weitere Lage $M n^{4^{+}-\delta}$ entlang der (100) Ebene einfügt. Diese Struktur wird als metastabil verstanden, da sie aufgrund des Hebelgesetzes als eine Mischung aus kubischer Pnma $x=0,66$ Phase und der monoklinen $x=0,85$ Phase angesehen werden muss [28] und kann auch als sogenannte C-Phase auftreten, die keine Ladungsordnung zeigt [33|. Des Weiteren ist in der CO-Phase auch orbitale Ordnung zu finden. Dies beruht auf der Tatsache, dass die $M n^{3^{+}+\delta}$-Ionen die entlang der (010) Ebene liegen über den Superaustausch die Besetzung der $3 z^{2}-r^{2}$-Orbitale bevorzugen. Unterhalb der Neel-Temperatur liegt ein C-Typ Antiferromagnet vor.

\subsubsection{Modelle für die Ladungsordnung und Korrelation mit realen Strukturen}

Das Phänomen der Ladungsordnung tritt typischerweise in stark isolierenden Systemen auf und wird durch starke Coulomb-Wechselwirkung verursacht. Hierbei ist der Abstand der Ionen die die Ladung tragen von entscheidender Bedeutung, sowie die Leitfähigkeit des Materials [34]. In den Manganaten sind beide Kriterien verletzt. Sowohl gibt es Ladungsordnung in den unterschiedlichsten Manganaten (PCMO,LCMO,NSMO) [27], deren Ionengröße und somit auch Abstand der Manganionen von einander variiert, als auch in Systemen mit extrem niedrigem Widerstand wie LCMO [7]. Die Ladungsordnung in den Manganaten ist dementsprechend von komplizierterem Ursprung als eine simple Coulombabstoßung. Im LCMO System unterscheidet man zwei Sorten von Ladungsordnung, deren Ausprägung sich deutlich unterscheidet. Die erste Art der Ladungsordnung tritt für Dotierungen $0,45 \leq x \leq 0,55$ auf und ist gekennzeichnet durch einen Phasenübergang erster Ordnung 17, 35, 36. Hierbei tritt eine partielle Ladungsordnung der Elektronen auf den Manganionen auf, sodass die $M n^{3^{+}+\delta}$ und $M n^{4^{+}-\delta}$ ein regelmäßiges Gitter entlang der a-Achse bilden. Der Übergang kann in Magnetisierungsmessungen als Peak in der Magnetisierung und in der spezifi- 
schen Wärme sichtbar gemacht werden und zeigt eine große Temperatur- und Magnetfeldhysterese. Mithilfe von Elektronenbeugung kann die Überstruktur entlang der a-Achse durch Überreflexe eruiert werden [35, 37. Der Übergang ist oft vom gleichzeitig auftretendem Ferromagnetismus überdeckt, sodass für die Betrachtung der magnetischen Messungen häufig Proben mit $\mathrm{x}=0,55$ verwendet werden. Hierbei kann aber nur in seltenen Fällen eine Hysterese beobachtet werden $|38-40|$. Für den Fall $\mathrm{x}=0,5$ wird oft von einer Mischung aus ,site-centered“ und „,bond-centered“ $\mathrm{CO}$ ausgegangen und somit das Bild der Zener-Polaronen benutzt [41].

Wenn die Dotierung weiter erhöht wird ändert sich die Art der Ladungsordnung drastisch. Die Ladungsordnung kann weiterhin als Peak in der Magnetisierung und auch in der Wärmeleitfähigkeit beobachtet werden, zeigt allerdings keine Hysterese mehr und der Phasenübergang ist nun zweiter Ordnung. In der Literatur werden zwei Szenarien diskutiert, die damals von Mori und Cheong entwickelt wurden, um die Verteilung der Ladungen zu beschreiben [27, 35, 36]: 1. Das Bi-Stripe-Szenario und 2. Das Wigner-CrystalSzenario.

Bei dem Bi-Stripe-Modell wird davon ausgegangen, dass die $\mathrm{Mn}^{3+}{ }_{-}$-Ionen in Streifen entlang der b-Richtung auftreten, verbunden durch ein $\mathrm{Mn}^{4+}$ Ion. Durch die Änderung der Ladungsordnung von einer punktzentrierten zu einer bindungszentierten Ladungsordnung muss außerdem die Ausrichtung der $e_{g}$-Orbitale betrachtet werden. Die beiden $e_{g}$-Orbitale zeigen auf das $\mathrm{Mn}^{4+}$-Ion und bilden einen rechten Winkel zueinander. Zwischen diesen Streifen bildet sich eine Kontrastphase, die nur aus $M n^{4+}$-Ionen besteht. Die Anzahl der Lagen dieser Phase folgt zwangsweise aus der Dotierung x mit $\left(2 \cdot \frac{x}{1-x}-1\right) L a_{1-x} C a_{x} M_{n} O_{3}$. Damit ergibt sich für $\mathrm{x}=0,66$ eine Überstruktur von $a=3 a_{0}$ und für $\mathrm{x}=0,75$ eine Überstruktur mit $a=4 a_{0}$, sowie für $\mathrm{x}=0,85$ $a=6 a_{0}$. Das bedeutet, dass sich eine immer größere Überstruktur bildet und die Brillouin-Zone damit immer kleiner wird.

Für sogenannte inkommensurable Dotierungen, die zwischen den oben genannten Dotierungen liegen, bilden sich nach dem Hebelgesetz verteilt die beiden nächstliegenden Phasen. Generell können in den TEM Bildern aber auch andere Überstrukturen sichtbar gemacht werden, selbst für Materialien mit einer kommensurablen Zusammensetzung [35, 42]. Dies ist der Tatsache geschuldet, dass schon einfache Versetzungen oder Fehlstellen die Grundzustandsenergie soweit verschieben können, dass sich eine andere Phase ausbilden kann. Generell müssen die Proben auf der Nanoskala als phasensepariert betrachtet werden [5, 35, 43]. Das Bi-Stripe-Szenario wird von vielen neueren Veröffentlichungen als Fehler in der damaligen TEM-Messung von Mori, Chen und Cheong gesehen [35, 44]. Die Verkleinerung der Gitterkonstante 
im Bereich außerhalb der Bi-Stripes von 0,9 [35, 45] spricht zwar generell für das Bi-Stripe-Modell, zu erwarten ist eine kleinere Gitterkonstante, die Größe von $\Delta a=0,9$ jedoch ist zu groß und lässt daher eher auf Abberation oder andere Aufnahmefehler des TEM schließen. Weitere TEM-Messungen haben daher konsequent das Wigner-Modell bestätigt [46, 47].

Die in Abbildung 7 gezeigten Strukturen sind die Bi-Stripe-Struktur nach Mori, Chen und Cheong [42], sowie die verzwillingte Struktur, die nach eigener Konstruktion entstand. Die ursprüngliche Struktur wurde um $90^{\circ}$ gedreht und entlang der Zwillingsgrenze, die (101)-Achse des Kristalls, mit der ursprünglichen Struktur zusammengefügt.

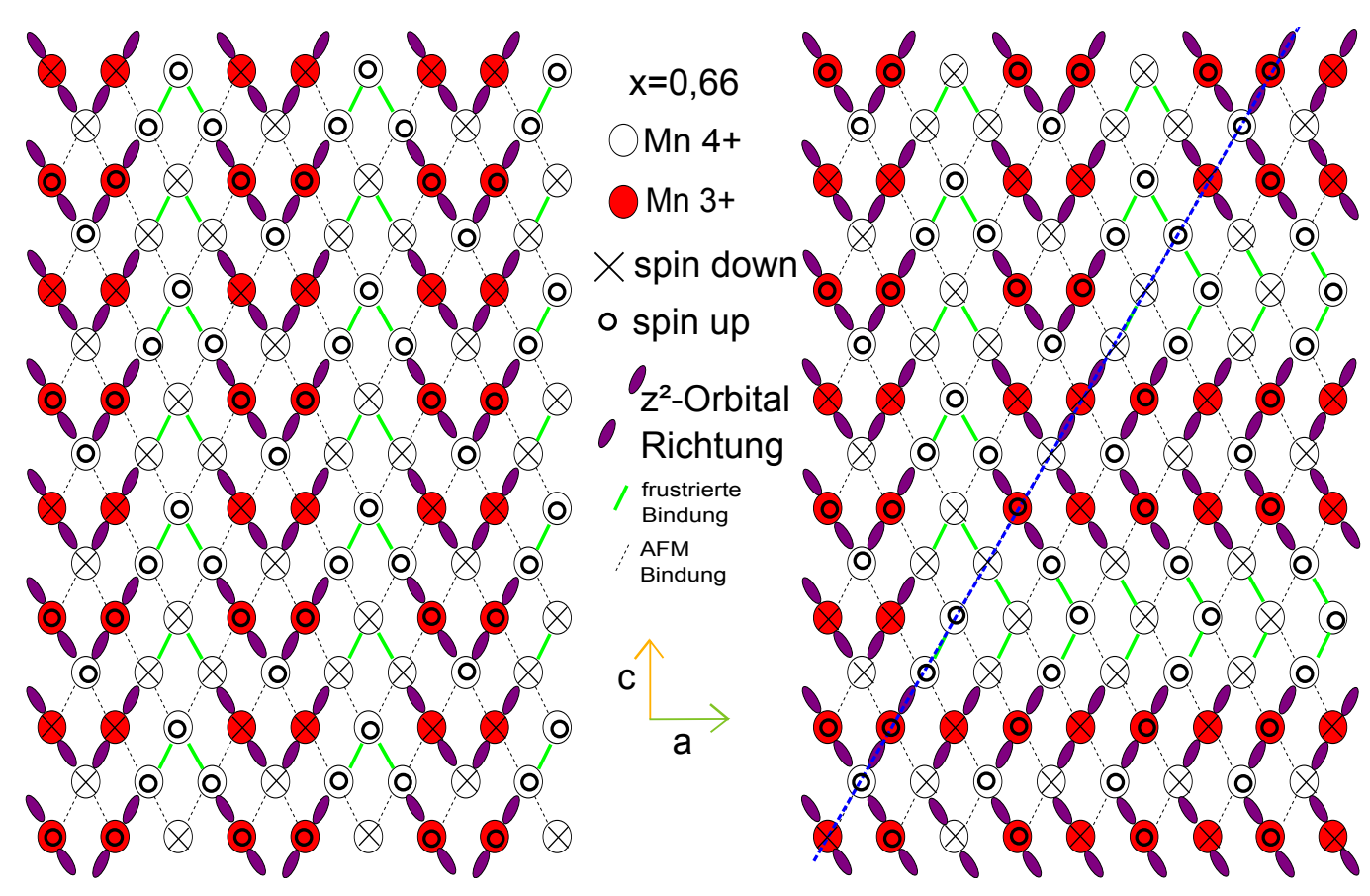

Abbildung 7 - Anordnung der $\mathrm{Mn}^{3+}$ und $\mathrm{Mn}^{4+}$ sowie die Ausrichtung der $z^{2}$-Orbitale für das Bi-Stripe-Modell. Außerdem eingezeichnet sind frustrierte und saturierte Bindungen, über grüne durchgezogene und schwarze gestrichelte Linien. Der Spin der Ionen ist in Zick-Zack-Ketten angeordnet [42].

Im Bild rechts ,durch die blaue gestrichelte Linie dargestellt, ist eine Zwillingsgrenze und die daraus resultierende Anordnung der $\mathrm{Mn}^{3+}$ und $M n^{4+} z u$ sehen. Die Struktur wurde hierbei erzeugt durch Drehung der ursprünglichen Struktur um $90^{\circ}$ und übereinanderlegen entlang der [101] Richtung 
Das „Wigner-Crystal“ Modell, das eigentlich aus der Theorie unreiner Metalle stammt [48], verwendet die gegenteilige Annahme, dass die $\mathrm{Mn}^{3+}{ }_{\text {-Ionen }}$ einen möglichst großen Abstand voneinander haben möchten, aufgrund der Coulombabstoßung. Auch in dieser Theorie ist es aber nötig, dass sich die $\mathrm{Mn}^{3+}$-Ionen entlang der b-Achse anordnen. Entlang der a-Achse haben die $\mathrm{Mn}^{3+}$-Ionen nun aber immer denselben Abstand und bilden damit genau wie im Fall des Bi-Stripe-Modells eine Überstruktur entlang der a-Achse. Für die Dotierung $\mathrm{x}=0,5$ gibt es keinen Unterschied für die Überstruktur beider Modelle. Für größere Dotierungen hingegen ergibt sich für die beiden Modelle eine unterschiedliche Verteilung der $M n^{3+}$ und $M n^{4+}$-Ionen. In beiden Modellen kann in TEM-Bildern, unterhalb der Ladungsordnungstemperatur, eine streifenartige Struktur sowohl im bright-field als auch dark-field aufgelöst werden [35, 49]. Weiterhin erwähnenswert ist allerdings, dass auch oberhalb der Ladungsordnungstemperatur eine Streifenstruktur sichtbar ist [50|. Die Änderung beim Unterschreiten der Ladungsordnungstemperatur besteht darin, dass der Abstand der Streifen sich abhängig von der Dotierung vergrößert. Der Grund für den Kontrast in den TEM-Messungen ist bis jetzt nicht geklärt. Was sich aber sagen lässt ist, dass das Bi-Stripe-Szenario im Gegensatz zum Wigner-Crystal Modell unterschiedliche Mechanismen benötigt um den Kontrast in $\mathrm{x}=0,5$ und $\mathrm{x}=0,66$ zu erklären, während für das WignerModell ein und derselbe Mechanismus verantwortlich sein sollte. In Abb. 8 ist die Struktur des Wigner-Crystal Modells gezeigt, sowie die verzwillingte Struktur, die nach eigener Konstruktion entstand. Die ursprüngliche Struktur wurde auch hier um $90^{\circ}$ gedreht und entlang der Zwillingsgrenze, die (101)-Achse des Kristalls, mit der ursprünglichen Struktur zusammengefügt. 


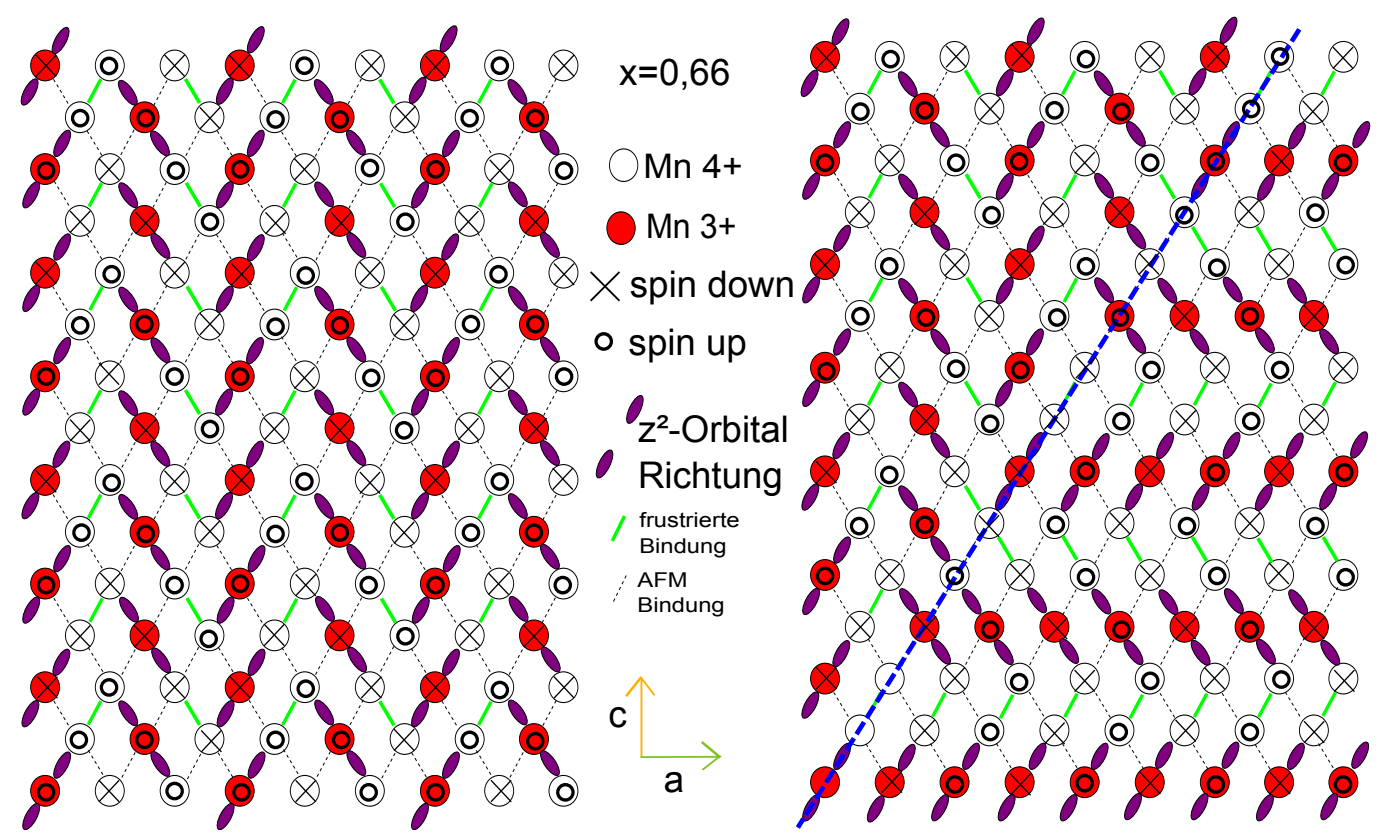

Abbildung 8 - Anordnung der $M n^{3+}$ und $M n^{4+}$ sowie die Ausrichtung der $z^{2}$-Orbitale für das Wigner-Crystal-Szenario. Außerdem eingezeichnet sind frustrierte und saturierte Bindungen, über grüne durchgezogene und schwarze gestrichelte Linien. Der Spin der Ionen ist in Zig-ZagKetten angeordnet [35].

Im Bild rechts in blau eingezeichnet ist außerdem eine (011) Grenzfläche und die daraus resultierende Anordnung der $\mathrm{Mn}^{3+}$ und $\mathrm{Mn}^{4+}$. Die Struktur wurde hierbei erzeugt durch Drehung der ursprünglichen Struktur um $90^{\circ}$ und übereinanderlegen entlang der [101] Richtung 
Die Ladungsordnung kann sich neben der Magnetisierung und spezifischen Wärme auch im Widerstand der Proben manifestieren, nämlich durch die starke Lokalisierung der Ladungsträger unterhalb der Ladungsordnungstemperatur als Plateau im Widerstand. Dieses Widerstandsplateau taucht typischerweise bei Messungen des Widerstands an Einkristallen und Polykristallen auf [49,51]. Für Filme wird es hingegen eher nicht beobachtet [27,52]. Eine mögliche Erklärung stellt hierbei das elektrische Feld dar, dass an die Proben angelegt wird und aufgrund der Größe der Filme typischerweise größer ausfällt als für Polykristalle. Das elektrische Feld hat dabei Einfluss auf die Ladungsordnung [27|. Zusätzlich kann die Ladungsordnung auch durch den Strom unterdrückt werden, da die elektrische Energie ausreicht um lokal die Ladungsordnung aufzubrechen und einen leitfähigen Pfad zu erzeugen |53|. Somit kann das Plateau im Widerstand nicht als alleiniges Kriterium eingeordnet werden um zu eruieren, dass eine Probe Ladungsordnung zeigt. Wichtiger scheint hingegen, das erhöhte magnetische Moment am Ladungsordnungsübergang und die Überstruktur zu sein. Eine weiterhin beliebte Variante den CO-Übergang zu bestimmen ist eine Auftragung der Temperatur gegen $\frac{d l n(\rho)}{d T^{-1}}$, hier zeigt sich die Ladungsordnung als Peak [51,52]. 


\section{3 Überstrukturen}

Es gibt typischerweise drei Arten von Überstrukturen. Atomare Überstrukturen, elektronische Überstrukturen und magnetische Überstrukturen. Die für die Arbeit wichtigen Überstrukturen sind elektronische. Wie bereits im vorherigen Kapitel erwähnt, erzeugt die Ladungsordnung im LCMO-System eine Überstruktur entlang der a-Achse, die durch Elektronenbeugung sichtbar gemacht werden kann. Diese elektronische Überstruktur hat weitere Auswirkungen auf die Bandstruktur. Die Brillouin-Zone wird aufgrund der Überstruktur auf den $\Gamma$-Punkt zurückgefaltet. Dadurch werden die longitudinal akustischen Moden optisch aktiv, da ihre Energie nun auf den $\Gamma$-Punkt zurückgefaltet unterschiedlich von null ist. Diese Tatsache lässt die Überstrukturen der unterschiedlichen kommensurablen Dotierungen als Peaks der Zustandsdichte in den THz-Messungen auftauchen. Das heißst wir können auch ohne Beugungstechniken die Überstruktur sichtbar machen, durch das Vorhandensein von Moden zwischen dem optischen und akustischen Zweig. Der Grund hierfür ist, dass die Energie der Photonen so gering ist, dass quasi nur um den $\Gamma$-Punkt herum gestreut wird. Die longitudinal akustischen Moden liegen jedoch zu niedrig um sie mit optischer Spektroskopie aufzulösen. Erst die Faltung ermöglicht es die Moden sichtbar zu machen.

\subsection{Boson-Peak}

Der Boson-Peak ist ein Phänomen, das in nahezu allen glasartigen oder ungeordneten Systemen auftritt, ein Peak der Zustandsdichte kurz unterhalb der ersten van-Hove-Singularität. Die van-Hove-Singularität beschreibt dabei die Unstetigkeit der Ableitung der Zustandsdichte, die 1953 das erste Mal für die lineare Kette von Léon van-Hove berechnet und beschrieben wurde [54]. Für den Boson-Peak wird oft die reduzierte Zustandsdichte betrachtet, nämlich eine Auftragung der Zustandsdichte geteilt durch die Kreisfrequenz zum Quadrat $\frac{g(\omega)}{\omega^{2}}$. Die reduzierte Zustandsdichte gibt damit die Anzahl der Zustände in einem Energieintervall an. Die Zustandsdichte ist im Debyemodell proportional zum Quadrat der Kreisfrequenz, daher wird oft die reduzierte Zustandsdichte aufgetragen um die Frequenzabhängigkeit zu berücksichtigen. Die van-Hove Singularität beschreibt nun gerade den Rand der BrillouinZone, an dem es in einer Dimension eine Divergenz der Zustandsdichte gibt. In drei Dimensionen gibt es nur eine Unstetigkeit und es divergiert nur die Ableitung. 
Wenn man nun von einem Kristall zu einem Glas übergeht, führt man typischerweise eine $\mathrm{zu}-$ fällige Verteilung der Kraftkonstanten ein. Damit wird die Unstetigkeit der Zustandsdichte zur typischen Form des Bosonpeaks. Die Höhe des Peaks wird dabei größer, je größer die Schwankung der Kraftkonstanten angenommen wird. Diese vermeintlich größere Intensität ist aber nur durch den Skalierungsfaktor $\omega^{2}$ bedingt. Die Anzahl der Moden die zum Peak beitragen ist gleich. Es gibt also keine zusätzlichen Moden die zum Bosonpeak beitragen [56, 57. Es ist auch möglich den Boson-Peak über eine kurzreichweitige Wechselwirkung zu modellieren. Die Höhe des Peaks und seine Position ist dabei vom Grad der Unordnung im System abhängig und kann durch einen Wechselwirkungsparameter J charakterisiert werden. Je größer J, desto größer die Wechselwirkung der ungeordneten Bereiche mit einander und desto weiter unterhalb der ersten vanHove-Singularität liegt der Peak und desto größer die Intensität. Damit ist die Intensität des Boson-Peaks in ungeordneten Systemen ein charakteristisches Merkmal für die Stärke der Unordnung des Systems. Die reduzierte Zustandsdichte ist dabei deshalb so interessant, weil sie durch optische Spektroskopie direkt zugängig ist. Wird zum Beispiel $\epsilon^{\prime \prime}(\omega)$ gemessen, so ist dies eine Repräsentation der reduzierten Zustandsdichte, da $\epsilon^{\prime \prime}(\omega) \propto \frac{D(\omega)}{\omega^{2}} \mid 58$. 


\section{Experimentelle Methoden}

\subsection{Schichtpräparation}

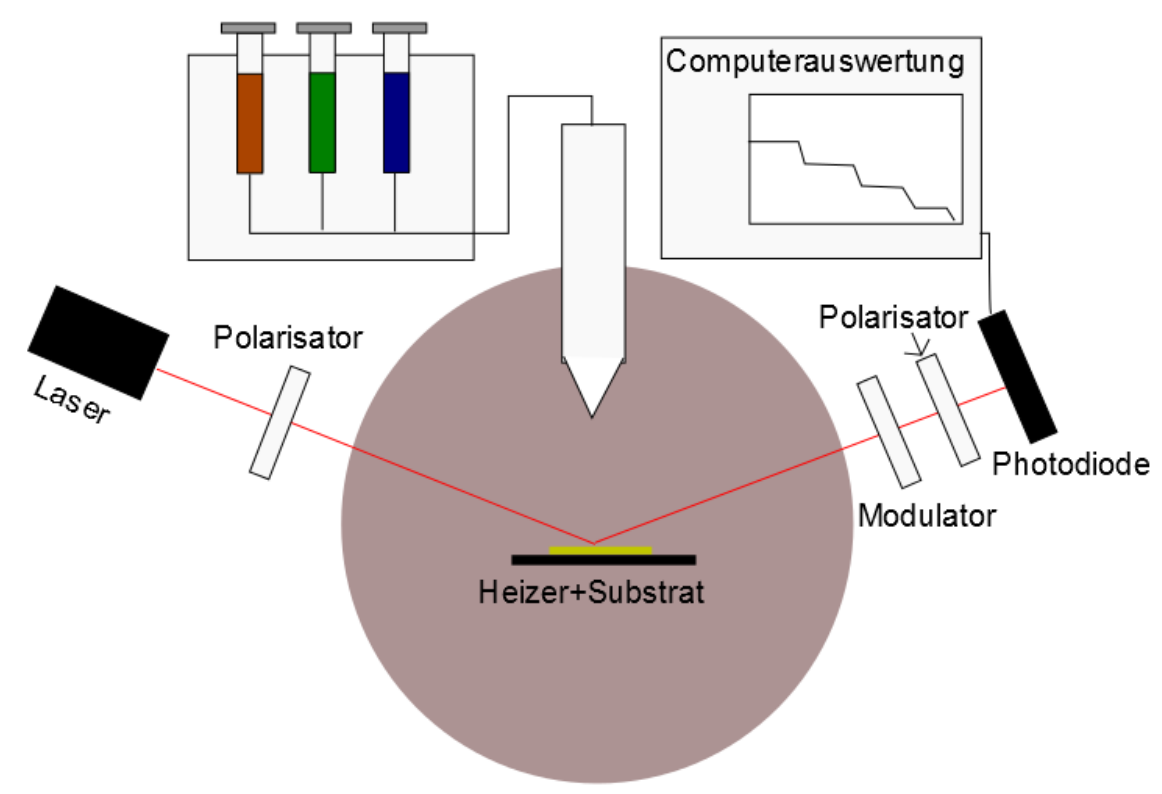

Abbildung 10 - Schematische Zeichnung der MAD3. Über Spritzendosierer wird eine Metall-acetylacetonat-Lösung durch die Lavaldüse gedrückt und über die Pressluft feinverteilt. Der Metallligand formt zusammen mit dem Sauerstoff der Umgebung die Schicht. Die Überwachung des Wachstums erfolgt über Ellipsometrie mithilfe eines He-Ne-Lasers

Die Metallorganische Aerosol Deposition (MAD) ist eine Methode zur chemischen Abscheidung von Oxiden aus der Gasphase. Das Prinzip der MAD soll nun im Weiteren illustriert werden. Eine Präkursormischung die zuvor in einer Handschuhbox abgewogen wurde, wird mit der entsprechenden Menge Dimethylformamid (DMF) vermischt. Die Lösung wird für einige Minuten im Ultraschallbad bei $30^{\circ} \mathrm{C}$ geschallt. Auf einem Siliziumcarbidheizer wird mit einer Messingklammer ein Substrat befestigt. Dieses Substrat wird nun mit einer Stromquelle auf die für die Deposition benötigte Temperatur erhitzt, wobei diese typischerweise zwischen $500^{\circ} \mathrm{C}$ und $1200^{\circ} \mathrm{C}$ variiert. Die Temperatur wird über ein Zweikanalpyrometer kontrolliert und auch während der Deposition beobachtet. Die Präkursorlösung wird bei Erreichen der nötigen Temperatur durch eine Pumpe in eine Lavaldüse gepresst, die an Druckluft angeschlossen ist. Hierbei wird allerdings nicht hauptsächlich auf 
den anliegenden Druck, sondern auf den typischen Durchfluss durch die Düse geachtet, der ebenfalls über einen Flusswächter überwacht wird, typische Werte sind hier $1-1,3 \mathrm{~m}^{3} / \mathrm{min}$. Das Aerosol wird durch diesen Vorgang auf das erhitzte Substrat gesprüht, wo die Pyrolysereaktion einsetzt, die den organischen Präkursor zersetzt in $\mathrm{CO}_{2}$ und $\mathrm{H}_{2} \mathrm{O}$. Hierbei bleibt der Metallligand übrig, der nun auf dem Substrat das Oxid bildet, das sich mit dem Substrat verbindet. Die MAD hat dabei den Vorteil, dass anders als bei Pulse-Laser-Deposition (PLD) oder Sputteranlagen, die Teilchen keine hohe Energie aufweisen, wenn sie das Target treffen, da die Pyrolysereaktion schon oberhalb des eigentlichen Substrats einsetzt. Dadurch können Fehlstellen und Versetzungen vermieden werden, die sonst zwangsweise auftreten, wenn die Teilchen mit hoher kinetischer Energie auftreffen, wie in den beiden andern Verfahren. Während der Deposition wird der Präkursor vollständig verbrannt und das Restgas konstant abgesaugt, wodurch sich keine Abscheidungen von Kohlenstoff oder andere Verunreinigungen bilden können. Die Deposition wird ebenfalls anders als bei PLD oder Sputteranlagen bei Normaldruck durchgeführt, was das Abpumpen der Anlage unnötig macht und somit die Geschwindigkeit mit der Proben hergestellt werden können drastisch erhöht. Außerdem sorgt der Normaldruck dafür das kein weiteres Auslagern der Proben bei Sauerstoff im Ofen notwendig ist, da während der Deposition mehr als genug Sauerstoff zur Verfügung steht. In dieser Arbeit wurde die MAD erstmalig dazu verwendet sehr dicke Schichten herzustellen. Die typischen Schichtdicken für Proben die in der MAD hergestellt werden sind 5-80nm, in dieser Arbeit konnte gezeigt werden, dass die MAD auch für Schichtdicken jenseits von $1 \mu m$ einsetzbar ist und somit für Anwendungen wie „coated conductors“ geeignet sein kann. 


\subsection{Strukturelle Untersuchungen}

\subsubsection{Röntgendiffraktometrie}

Um die Qualität der fabrizierten Proben $\mathrm{zu}$ untersuchen, sind Röntgendiffraktometriemessungen (XRD) unabdingbar. Hierbei wurden zwei Vollschutzgeräte von Siemens verwendet. Die D5000, eine Röntgenröhre mit Kupferanode und Graphitmonochromator, die eine Gesamtleistung von bis zu 1600W hat und besonders für Polykristalle und Pulverproben geeignet ist. Sowie die D8 ebenfalls eine Rönt-

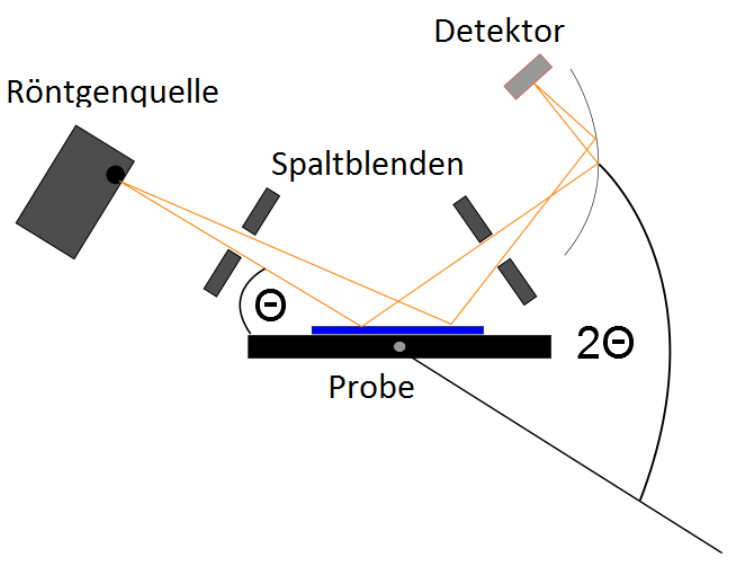

Abbildung 11 - Bragg-Brentano-Geometrie für die D8. Über den Göbelspiegel wird die $k_{\beta}$-Linie herausgefiltert genröhre mit einer Kupferanode deren Maximalleistung bei 2400W liegt, die aber im Normalbetrieb bei denselben Werten für Strom und Spannung betrieben wird, wie die D5000, da der Göbelspiegel, der in der D8 verwendet wird, auf diese Werte geeicht ist. Die D5000 besitzt einen rotierbaren Probenhalter wodurch auch $\Phi$-Scans möglich sind. Hierbei wird zumeist der Peak mit der höchsten Intensität angefahren und der Winkel des Detektors um $\Psi$ verkippt. Wird nun über den drehbaren Probentisch der Winkel $\Phi$ durchgefahren ergeben sich je nach Symmetrie der untersuchten Probe Peaks abhängig vom Winkel $\Phi$. Die D5000 ist nur mit Schneidblenden ausgerüstet, diese schränken den Strahl jedoch nur vertikal ein. Für einen $\Phi$-Scan ist es hingegen nötig den Strahl auch horizontal einzuschränken. Durch das Fehlen des vierten Goniometerkreises und da der Strahl nicht eingeschränkt werden kann zeigen die azimuthalen Scans extrem breite Peaks. Ist es sonst prinzipiell möglich aus den azimuthalen Scans die Verteilung und Mosaizität einer Probe zu bestimmen ist dies aufgrund der inhärenten Beschränkungen mit der D5000 nicht möglich. Die D5000 kann daher nur für die Bestimmung der Symmetrie benutzt werden und um zu zeigen, dass LCMO entlang der Achsen des MgO aufwächst, wenn die Peaks des LCMO bei denselben Winkeln wie die des $\mathrm{MgO}$ liegen. Jegliche Aussagen über Mosaizität oder Kristallinität sind auf- 
grund der fehlenden Blende nicht möglich. Allerdings ist bei der D5000 die maximale Intensität aufgrund der Verwendung des Graphitmonochromators begrenzt und um einen Faktor 4 geringer als bei der D8, die außerdem einen besseren Detektor hat der als Maximalwert $10^{6}$ counts $/ s$ messen kann. Beide Diffraktometer benutzen die Bragg-Brentano-Geometrie. Hierbei ist die Röntgenröhre fest montiert. Der Probenhalter ist drehbar und der Detektorarm kann über den Bereich von $0-120^{\circ}$ (D5000)/0-100(D8) schwenken. Die Bragg-Brentano-Geometrie bedeutet, dass sich die Probe immer im Fokuspunkt befindet und der Detektor einen Winkel von $2 \Theta$ zur Quelle aufweist. Der Probenhalter ist mit dem Winkel $\Theta$ gegen die Quelle gedreht weshalb diese Geometrie auch oft als „ $\Theta-2 \Theta$ Geometrie“ bezeichnet wird. Über die Braggformel kann die Gitterkonstate des Films a bestimmt werden.

$$
n \cdot \lambda=2 \cdot d \cdot \sin (\Theta)
$$

Hierbei ist $d_{h k l}=\frac{a}{\sqrt{h^{2}+k^{2}+l^{2}}}$ der Netzebenenabstand und $\mathrm{h}, \mathrm{k}, \mathrm{l}$ die Miller Indizes. Für eine genauere Berechnung die auch die Intensität einzelner Peaks berücksichtigt ist der Struktur- und Formfaktor nötig. Der Strukturfaktor F berechnet sich durch die atomaren Streufaktoren $f_{i}$ wie folgt:

$$
F_{h k l}=\sum_{i} f_{i} \exp \left(i \vec{G} \vec{r}_{i}\right)
$$

$\vec{G}$ ist hierbei der reziproke Gittervektor und $\overrightarrow{r_{i}}$ der Richtungsvektor eines jeden Elements.

Es wurde in dieser Arbeit sowohl die D8 als auch die D5000 benutzt, zweitere hauptsächlich für $\Phi$-Scans um zu bestimmen ob die Schichten einkristallin aufwachsen. Die Diffraktogramme der D8 sind im Allgemeinen unsauberer als die der D5000, insofern als $\beta$-Peaks des charakteristischen Spektrums aufgrund des Göbelspiegels nicht komplett herausgefiltert werden können. Andererseits ist die Intensität der Strahlung sowie die Güte des Detektors der D8 entscheidend besser. Diese zusätzlichen, ungewollten Peaks in den Röntgenspektren können also leicht identifiziert und vernachlässigt werden, wohingegen die Identifizierung von möglichen Verunreinigungen und Peaks zusätzlicher Orientierungen erleichtert wird. 


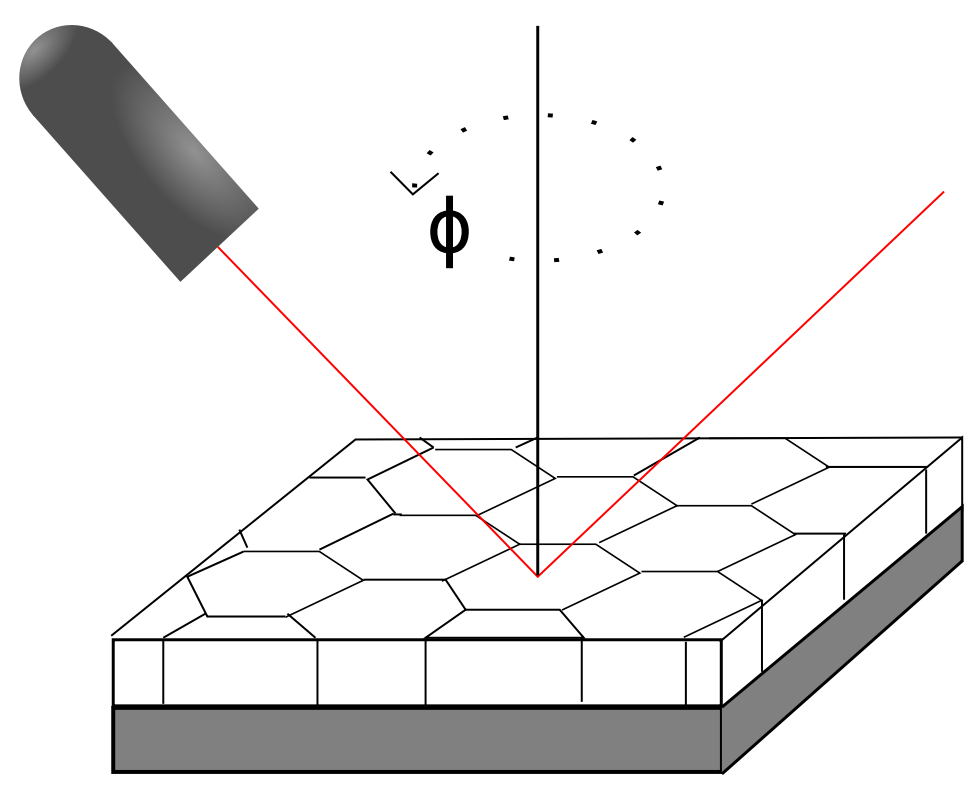

Abbildung 12 - Unter festen Winkeln $\Theta$ und $2 \Theta$ wird die Probenebene gedreht um die Achsenzähligkeiten und Mosaizität zu bestimmen 


\subsubsection{Transmissionselektronenmikroskopie}

Transmissionselektronenmikroskopie (TEM) ist eine Mikroskopietechnik, bei der ein gebündelter Elektronenstrahl auf eine dünne Lamelle eines zuvor präparierten Materials gelenkt wird und über das Beugungsbild der Elektronen sehr genaue Aussagen über die kristalline Struktur gemacht werden können. Die Elektronen sollen dabei die Lamelle vollständig durchstrahlen. Damit ist die Dicke der Lamelle meist über die Beschleunigungsspannung limitiert auf einige nm. Die TEM Messungen in dieser Arbeit wurden von Vladimir Roddatis aus dem Institut für Materialphysik der Universität Göttingen durchgeführt und ausgewertet. Das verwendete Mikroskop ist das E-TEM. Die zurückgestreuten Elektronen können, durch unterschiedliche Detektoren nach Energien aufgeschlüsselt, aufgenommen werden. Hierbei gibt es typischerweise drei wichtige Bereiche:

Bright-Field (BF): Hierbei werden die Elektronen unterhalb des Elektronenstrahls detektiert. Das Beugungsbild der Elektronen zeigt nach Fouriertansformation die reale Gitteranordnung. Detektiert werden hier die nicht gestreuten Elektronen.

Dark-Field (DF): Der Hauptstrahl wird geblockt und die Elektronen werden mit einem Ringdetektor detektiert. Die detektieren Elektronen bestehen damit hauptsächlich aus inkohärent gestreuten Elektronen. Diese Elektronen sind deutlich sensitiver auf Grenzflächen und Unstetigkeiten in der Probe und können daher verwendet werden um Defekte in der Probe sichtbar zu machen.

High angle annular dark field (HAADF): Der Ringdetektor für HAADF liegt noch weiter außerhalb als der DF-Detektor und ist dafür gedacht die stark, inkohärent gestreuten Elektronen aufzunehmen. Diese Elektronen zeigen einen starken Z-Kontrast und sind damit besonders gut geeignet um die chemische Struktur von Proben aufzunehmen. Wenn der HAADF-Detektor groß genug ist wird er außerdem benutzt um EELS für Elektronenenergieverlustspektroskopie durchzuführen. Hierbei werden die spezifischen Energien der einzelnen Elemente der Probe untersucht und dadurch eine Karte der chemischen Verteilung erstellt.

Des Weiteren muss zwischen TEM und Scanning-TEM (STEM )unterschieden werden. Hierbei wird die Optik des Mikroskops quasi invertiert. Die Bilder werden nun seriell aufgenommen. STEM-Aufnahmen zeigen daher oft mehr Rauschen und benötigen länger für die Aufnahme. Allerdings ist der ZKontrast in den STEM-Aufnahmen deutlich größer und es ist daher möglich auch chemische Elementkarten aufzunehmen. Alle hier erwähnten Abhängigkeiten sind nur zutreffend wenn die Elektronenverteilung eine analytische Form annimmt. Ist dies nicht der Fall können die Aufnahmen vom Einstrahl- 
winkel der Apertur und der Beschleunigungsspannung abhängen. Außerdem spielt die Dicke der Lamellen eine wichtige Rolle. So kann der vermeintliche Z-Kontrast auch durch schlechte Probenpräparation entstehen. Eine sehr vorsichtige und genaue Präparation aller Proben ist daher nötig damit verwertbare sowie vergleichbare Bilder entstehen.

\subsubsection{Rastertunnelmikroskopie}

Das Bruker Multimode STM ist ein kommerzielles Rastertunnelmikroskop (STM), das sich den Tunneleffekt für die Oberflächenmikroskopie zunutze macht. Hierbei wird eine leitende Spitze, in unserem Fall Platin-Iridium Draht über einer Probe fixiert. Über eine Piezoansteuerung, die an einen Proportional- und einen Integralregler angeschlossen ist, kann nun durch den Effekt des Tunnelstroms die Oberfläche leitender Proben vermessen werden. Hierfür wird die Spitze bis auf wenige nm an die Probe angenähert und eine elektrische Spannung zwischen Spitze und Probe angelegt. Ab einem gewissen Abstand können die Elektronen nun über die Barriere tunneln. Hält man nun über die beiden Regler den Strom konstant, kann durch die exponentielle Abhängigkeit des Tunnelstroms mit dem Abstand von der Schicht eine extrem genaue Vermessung der Oberfläche durchgeführt werden. 


\subsection{Magnetische Charakterisierung}

Wie schon am Phasendiagramm von LCMO ersichtlich ist, zeigen die LCMO Schichten einen Übergang vom paramagnetischem zum antiferromagnetischen Zustand, weshalb Magnetisierungsmessungen sowie Hysteresemessungen unabdingbar sind. Diese Messungen wurden im vorliegenden Fall mit einem SQUID Magnetometer MPMS von Quantum Design durchgeführt. Das maximale Feld das angelegt werden kann liegt bei $H=5 T$, in einem Temperaturbereich von $T=2-400 K$. Es wurden hierbei sowohl $\mathrm{M}(\mathrm{T})$ als auch $\mathrm{M}(\mathrm{H})$ Kurven aufgenommen. Das MPMS ist ein rf-SQUID, wobei das rf für radiofrequency steht. Diese besondere Art von SQUIDs weist eine höhere Genauigkeit als konventionelle DC-SQUIDs auf. Die Funktionsweise eines SQUID beruht dabei auf der Flussquantisierung und dem Josephson-Effekt. In einem supraleitenden Material bilden sich sogenannte Cooper-Paare, eine Überlagerung der Wellenfunktionen zweier Elektronen die den Effekt hat, dass sich das neue daraus entstehende Quasiteilchen als Boson verhält und somit nach Bose-Einstein in einen Zustand kondensieren kann. Diese sogenannte Bose-Einsteinkondensation sorgt für eine Erniedrigung der Energie des Elektronensystems unterhalb einer kritischen sogenannten Sprungtemperatur. Die Eigenschaften dieses supraleitenden Zustands sind unter anderem, dass sich das Material bei moderaten Feldern wie ein perfekter Diamagnet verhält. Dieser Effekt wird ausgenutzt um die Magnetisierung von Proben zu bestimmen. Hierbei wird die Probe zwischen ein supraleitendes Spulenpaar gebracht und dann entlang der Spulenachsen verfahren. Hierbei wird durch die Magnetisierung der Probe ein Abschirmstrom in den supraleitenden Spulen induziert. Der Widerstand eines Supraleiters ist null, dadurch ist es nicht möglich den Strom zu messen der fließt um den induzierten Strom zu bestimmen. Josephson fand damals eine Möglichkeit durch Tunnelkontakte dieses Problem zu umgehen. Seine Idee war dabei, dass man eine extrem dünne Zwischenschicht in einen supraleitenden Ring einbringt. Diese Schicht ist aus einem nicht supraleitenden Material und sorgt nun dafür, dass ein Widerstand abfällt. Wenn die Schicht dünn genug gewählt wird können die Cooper-Paare aber durch die Barriere tunneln. Man zerstört also nicht den supraleitenden Zustand. Damit war es nun erstmals möglich Änderungen der Magnetisierung von weniger als einem $\mu_{B}$ zu bestimmen. Im gegebenen Fall wird die supraleitende Spule in zwei Hälften geteilt und an diese Hälften über eine Induktivität eine Wechselspannung mit Frequenzen im $\mathrm{MHz}$ Bereich angelegt. Über diese vorgeschaltete Induktivität kann nun der abfallende Strom mit extrem großer Genauigkeit gemessen und somit die Magnetisierung bestimmt werden. 


\subsection{Transportuntersuchungen}

Das Quantum Design PPMS ist ein $H e^{4}$-Kryostat der die Möglichkeit bietet Widerstand oder Thermokraft sowie spezifische Wärme bei Temperaturen zwischen $T=2-400 K$ und magnetischen Feldern zwischen $\mathrm{H}=0-9 \mathrm{~T}$ zu messen. Das PPMS wurde in dieser Arbeit dafür verwendet die Widerstandsdaten in Abhängigkeit von Temperatur und Magnetfeld aufzunehmen. Die Probe wird hierfür auf einem speziellen Puck befestigt, der in Steckkontakte innerhalb des PPMS eingesteckt werden kann. Das PPMS fährt nun über ein vollautomatisiertes Steuerprogramm eine Temperaturrampe, während gleichzeitig der Widerstand in konstanten Temperaturschritten gemessen wird. Als Kontakte für die Proben wurden hierbei Kupferdrähte der Dicke $0,125 \mathrm{~mm}$ verwendet sowie Leitsilber zum Kontaktieren. Die Widerstandsmessungen wurden dabei in der Vierpunkt Geometrie vermessen [59. Bei dieser Messtechnik werden vier Kontakte in einer Linie auf der Probe angebracht die äußersten beiden Kontakte dienen hierbei dazu einen Strom durch die Probe zu schicken. Die inneren Kontakte, die einen sehr großen Innenwiderstand und einen Differenzenverstärker besitzen werden dafür verwendet die Spannung zwischen den beiden inneren Kontakten zu messen. Auf diese Art und Weise kann das Potential der Kontakte vernachlässigt werden und die Widerstandsmessungen zeigen keine Abhängigkeit von der Leitfähigkeit der Kontakte. 


\subsection{Optische Spektroskopie}

\subsubsection{Terahertz Spektroskopie}

Die THz- und FIR-Messungen, die in den nächsten beiden Unterkapitel behandelt werden, wurden von Elena Zhukova und Boris Gorshunov in Stuttgart durchgeführt. Bei der THz-Spektroskopie wird ein Mach-Zehnder-Interferometer verwendet wie in Abb. 13 zu sehen ist. Die THz-Strahlung wird hier von einem sogenannten Backward-Wave-Oscillator (BWO) erzeugt. Hierbei wird ein Elektronenstrahl durch eine Spule geschickt. Abhängig von der Beschleunigungsspannung entsteht auf der Spule nun eine elektromagnetische Welle, die entgegen der Richtung des Elektronenstrahls läuft. Diese elektromagnetische Welle sorgt für Dichtefluktuationen am Ende der Spule, die als THz Strahlung ausgekoppelt werden. Der Name BWO stammt also daher, dass die Welle auf der Spule rückwärts läuft. Da diese THz-Quellen mit der Anodenspannung durchstimmbar sind, eignen sie sich gut als Quellen für frequenzabhängige THz-Messungen. Klarer Nachteil dieser Technik ist die gemeinhin recht kleine Intensität der Strahlung und die geringe Laufzeit der Oszillatoren von wenigen hundert Arbeitsstunden. In dem Mach-ZehnderInterferometer ist in dem zweiten Strahlengang für den Referenzstrahl ein beweglicher Spiegel eingebaut. Dieser Spiegel vibriert mit einer konstanten Frequenz in einem $45^{\circ}$-Winkel zum Strahl. Diese Vibration soll stehende Wellen, die bei THz-Messungen sehr einfach auftreten können, verhindern. Bei sehr leitfähigen oder dicken Proben wird oft nur die Reflektivität gemessen, da eine Transmissivität nicht gemessen werden kann. Die Spektren werden dabei für viele verschiedene Frequenzbereiche mit unterschiedlichen Quellen gemessen und später mit einem Fitprogramm gefittet, um die Werte für die Leitfähigkeit zu ermitteln. Die THz-Strahlung sorgt im Allgemeinen für Oszillationen im Frequenzraum, die von der Schichtdicke des Materials und dessen Leitfähigkeit abhängen. Mit einem Fitprogramm(WASF) das in Stuttgart geschrieben wurde und bei bekannter Dicke der Schicht, kann nun die Leitfähigkeit bestimmt werden.

\subsubsection{Ferne-Infrarot-Spektroskopie}

Die Far-Infrared (FIR) Messungen werden mit einem Fouriertransform Infrarotspektrometer von Bruker durchgeführt. Anders als bei den THz-Messungen wird bei den FIR-Messungen kein Signal fester Frequenz eingekoppelt und gemessen (Frequenzdomäne) sondern ein zeitlich verteilter Puls von weißem Rauschen auf die Probe gesandt, um dann durch eine Fouriertransformation von der Zeitdomäne, in der man misst, wieder in die einfacher zu verstehende Frequenzdomäne zurückzugelangen. Diese Technik ist weit verbreitet, da sie 
es erlaubt mit nur einer einzigen Messung ein komplettes Spektrum aufzunehmen. Die Messungen werden dadurch erschwert, dass die Daten fouriertransformiert werden müssen um in den einfacher verständlichen Frequenzraum zurückzugelangen. Nun gibt es zwar sehr viele Algorithmen, die solch eine „Fast Fourier Transformation“ durchführen, allerdings ist es hierbei von großer Wichtigkeit wie groß der Bereich ist der fouriertransformiert wird. Alle FIR-Messungen die in dieser Arbeit gezeigt werden, wurden von Elena Zhukova und Boris Gorshunov in Stuttgart durchgeführt und ausgewertet, im Rahmen der Kooperation im Projekt DFG DR 228/36. Aufgrund des Alternativverbots bei der Raman-Spektroskopie ist es sehr wichtig auch IRSpektroskopie durchzuführen, um alle Schwingungsmoden eines Systems zu charakterisieren.

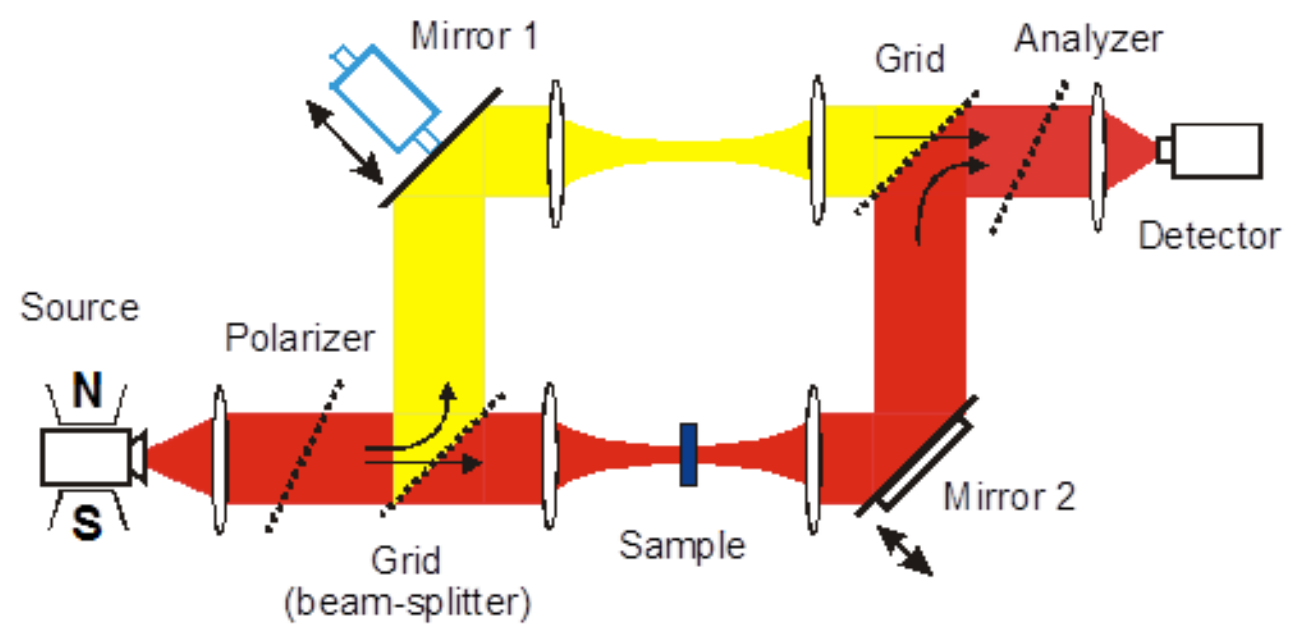

Abbildung 13 - Mach-Zehnder Aufbau des THz-Spektrometers in Stuttgart. Über die BWO-Quelle wird Terahertzstrahlung in den Aufbau ausgekoppelt und über Teflonlinsen fokussiert. Der mit Mirror1 gekennzeichnete Spiegel ist außerdem an eine Membran angeschlossen und vibriert mit einer leicht veränderlichen Frequenz. Damit sollen unter anderem stehende Wellen verhindert werden 


\subsubsection{Raman-Spektroskopie}

Das LabRAM HR Evolution von Horiba Scientific ist ein Ramanspektrometer mit Mikroskopaufsatz das mit zwei Lasern, einem 633nm Helium-Neon Laser und einem 532nm YAG-Laser, ausgestattet ist. Die Messungen werden hier in der sogenannten „backscattering geometry“ durchgeführt. Dies bedeutet, dass das Laserlicht und das ramangestreute Licht denselben Strahlenweg verwenden. Die Differenz in der Energie des angeregten Zustands zu der Energie des Grundzustands wird nun als Verschiebung von der Basislinie des Lasers von einer CCD detektiert, hierbei können nur die sogenannten Stokes-Prozesse beobachtet werden. Ein Stokes-Prozess bedeutet, dass das Energieniveau das durch die Laserillumination besetzt wird, einen höherenergetischen Zustand besetzt als das Grundniveau und somit die Differenz der Energien positiv ist. Damit die normalerweise exorbitant große Intensität des Laserstrahls nicht die Resultate komplett überstrahlt, wird ein sogenannter Edge-Filter verwendet, hierbei handelt es sich entweder um einen Multilagen Edge-Filter oder um einen holographischen Notch-Filter. Das Material aus dem der Edge-Filter besteht ist dabei von der Wellenlänge des Lasers abhängig und soll dafür sorgen, dass die Laserintensität die CCD-Kamera nicht erreicht. Die Güte des Edge-Filters bestimmt damit auch wie groß die minimale Wellenzahl ist, bei der man messen kann ohne ein Abschneiden der Messwerte durch den Filter zu riskieren, typische Werte liegen hier bei $100 \mathrm{~cm}^{-1}$ bei Verwendung eines Doppellagenfilters [60]. Bei der Raman-Streuung wird ein Material mit Laserlicht angeregt. Diese Anregung bewirkt mit einer gewissen Wahrscheinlichkeit eine Anregung der Elektronen aus dem Grundzustand Abbildung 14 - Energieschema von Stokesheraus in einen virtuellen Zustand. Dieser hö-

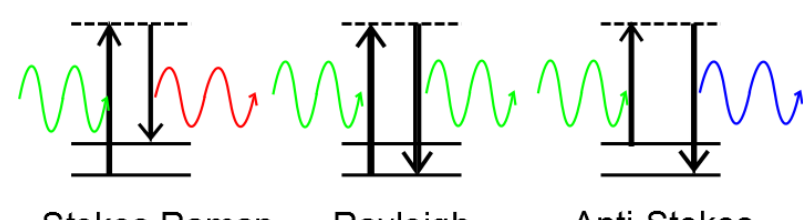

Stokes Raman
Rayleigh
Anti-Stokes

\section{Raman-Rayleigh- und Anti-Stokes-Streuung} herenergetische Zustand des Materials ist unter normalen Bedingungen instabil, sodass das Elektron nach der Anregung wieder zurück in das Grundniveau fällt. Die Elektronen fallen nach einer sehr kurzen Verweildauer von $\tau \approx 10^{-9} s$ zurück und können dies auf zwei Arten tun. Bei der einfachsten Variante, der sogenannten Rayleigh-Streuung, fällt das Elektron direkt in denselben Zustand zurück aus dem es angeregt wurde. Es gibt aber noch einen zweiten viel interessanteren Prozess, die sogenannte Raman-Streuung oder auch Stokes-Raman-Streuung. Hierbei wird das Elektron in einen vir- 
tuellen Zustand angehoben, fällt aber anstatt in den Grundzustand in einen höher energetischen Zustand zurück, um dann in einem weiteren Schritt den Grundzustand zu erreichen. Dies kann in einem Energiediagramm wie in Abb. 14 dargestellt werden. Bei diesem Prozess wird ein Photon geringerer Energie freigesetzt. Diese Energie kann nun exakt ausgezeichneten Schwingungsmoden des Materials zugeordnet werden. Nach dem Alternativprinzip gibt es zwei Arten von Schwingungen. Schwingungen, die die Polarisierbarkeit ändern: $I \propto\left(\frac{d \alpha}{d q}\right)^{2}$, hierbei ist $\alpha$ der Polarisierbarkeitstensor und q eine Normalkoordinate, oder Schwingungen, die das Dipolmoment verändern $I \propto\left(\frac{d \mu}{d q}\right)^{2}$, hier ist $\mu$ das Dipolmoment $|60|$. Erstere schwingen symmetrisch um das Symmetriezentrum und sind als Raman-Moden bekannt, zweitere schwingen asymmetrisch und sind Infrarot(IR)-Moden. Hierbei ist wichtig zu betonen, dass bei der Ramanspektroskopie in inversionssymmetrischen Kristallen keine IR-Moden auftauchen und umgekehrt. Misst man also beide, IR- und Ramanmoden ein und desselben Systems, ist eine vollständige Charakterisierung des Schwingungsspektrums möglich. Für die Raman- und IR-Spektroskopie ist es wichtig die Symmetriegruppe des untersuchten Materials zu kennen, da die Symmetriegruppen bestimmen, welche Moden IR-aktiv sind und welche ramanaktiv. Als Beispiel lässt sich das $\mathrm{CO}_{2}$ Molekül betrachten. Es zeigt drei mögliche Schwingungsmoden: eine symmetrische Schwingungsmode, bei der die Sauerstoffatome in entgegengesetzte Richtungen ausgelenkt werden und das Kohlenstoffzentrum ruht, sowie zwei unsymmetrische Moden, bei denen alle Atome eine Schwingung durchführen. Wie nicht anders zu erwarten ist die erste Schwingung ramanaktiv, da sich nur die Polarisierbarkeit durch Ausdehnung der Elektronenverteilung ändert, nicht aber das Dipolmoment, das konstant bleibt. Sowie die beiden anderen IR-aktiven Schwingungen, die durch die Änderung des Dipolmoments entstehen. Während also die Normalmoden von dreiatomigen Molekülen noch relativ einfach zu bestimmen sind, wird es, je größer die Basis von Atomen ist, immer schwieriger diese Normalschwingungen zu definieren. In Kristallgittern macht man sich zunutze, dass sich die Struktur eines Einkristalls durch das Prinzip der sogenannten Einheitszelle darstellen lässt. Wenn man nun die Normalschwingungen der Einheitszelle kennt, hat man damit alle Schwingungsmoden des Einkristalls determiniert. Hierfür gibt es verschiedene Ansätze, die von „Shell model“ und „local density approximation“(LDA) bishin zu „density matrix functional theory“(DMFT) reichen. Diese unterschiedlichen Ansätze sind notwendig, da die Intensitäten der harmonischen Schwingungen in einigen Symmetriegruppen extrem gering sind und somit anharmonische Terme in die Betrachtung einfließen müssen, um das wirkliche Spektrum berechnen zu können. Die Intensität einer Ramanmode lässt sich über den Raman-Tensor oder den 
Gradienten der Polarisierbarkeit berechnen:

$$
\begin{gathered}
I_{\text {Raman }}=K \cdot P_{\text {Laser }}\left(\nu_{0}-\nu_{i}\right)^{4}\left[\frac{d \alpha}{d q}\right]^{2} \\
I_{\text {Raman }}=A \cdot\left[\sum e_{i}^{\sigma} R_{\rho \sigma} e_{s}^{\rho}\right]^{2}
\end{gathered}
$$

Die erste Formel stellt hierbei die allgemeinere der beiden Formeln dar. K ist eine Proportionalitätskonstante, die eine unterschiedliche Größe für die unterschiedlichen Moden aufweist, $\mathrm{P}$ ist die Laserleistung und $\nu_{0}$ die Wellenlänge des Lasers. $\nu_{i}$ beschreibt die Frequenz der angeregten Schwingung sowie $\frac{d \alpha}{d q}$, wie bereits erwähnt, die Änderung der Polarisierbarkeit entlang der Normalkomponente q.

Die zweite Formel kann zur Beschreibung von Kristallsystemen verwendet werden. Hierbei wird der sogenannte Raman-Tensor verwendet. Die Werte des Raman-Tensors sind dabei die Ableitungen der Polarisierbarkeit, entlang der einzelnen Kristallrichtungen. Durch die Symmetrie des Kristalls lässt sich ein Normalsystem finden, typischerweise wird die Einheitszelle des Kristalls verwendet, in dem die Ableitungen entlang der Achsen, den Eigenwerten des Raman-Tensors entsprechen. Die $e_{i}^{\rho}$ und $e_{s}^{\sigma}$ benennen hier jeweils die Polarisationsrichtung des einfallenden Lichts und des ausfallenden Lichts, wobei $\rho$ und $\sigma$ für alle Richtungen x,y,z durchgespielt werden müssen. Die RamanTensoren für die einzelnen Punktgruppen lassen sich in mehreren Quellen finden [61,62.

Die mathematische Beschreibung des Ramanprozesses lässt sich damit auf ein Eigenwertproblem zurückführen. Im in dieser Arbeit verwendeten Aufbau wird ,wie bereits erwähnt, die sogenannte ,back-scattering“-Geometrie benutzt. Hierbei wird der Laserstrahl senkrecht auf die Probe geführt und die Ramanstreuung mit demselben Mikroskopaufbau abgegriffen. Der k-Vektor des einfallenden Lichts steht senkrecht auf der xy-Ebene und ist parallel zu z. Wenn das gestreute Licht nun unter einem Winkel $\Psi$ betrachtet werden soll, so ergibt sich für den E-Feldvektor der senkrecht auf $k_{s}$ steht die Form $e_{s}^{\|}=(\cos (\Psi), 0,-\sin (\Psi))$ und $e_{s}^{\perp}=(0,1,0)$. Der E-Feldvektor des einfallenden Lichts ist $e_{i}=\left(e_{i}^{x}, e_{i}^{y}, 0\right)$ Wir berechnen den Ramantensor nun über $e_{i} \cdot R \cdot e_{s}$. Damit wird deutlich, dass Komponenten der Ramanmoden in zRichtung durch den Sinus $\Psi$ unterdrückt werden und nur Schwingungen in der xy-Ebene im Messsignal auftauchen.

Weiterhin müssen wir auch die Drehung der Probe innerhalb der xyEbene berücksichtigen. Hierfür führen wir einen Vektor $\Theta$ ein, der die Drehung um die z-Achse beschreibt. Die Herleitung der Intensitäten kann nun entweder durch Einheitsvektoren entlang der um $\Theta$ gedrehten Richtungen berechnet werden, oder in dem man sich ins neue Probensystem begibt und den 


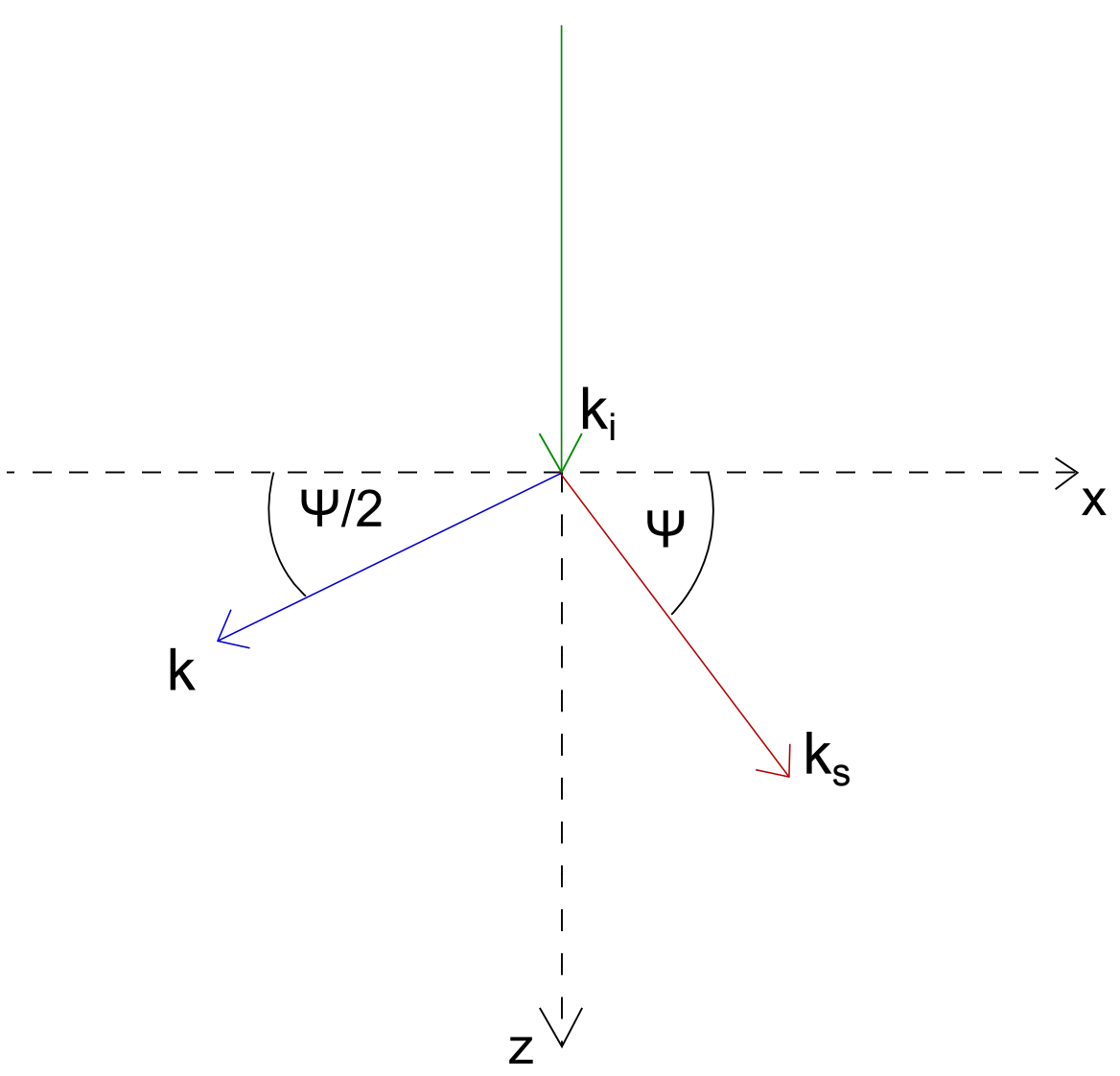

Abbildung 15 - Wellenvektoren des einfallenden Laserlichts $k_{i}$, des gestreuten Lichts $k_{s}$ und des Phonons $k$

Raman-Tensor im veränderten Koordinatensystem darstellt [63]. Beide Darstellungen sind natürlich äquivalent. Durch Verzwillingung der Probe wird das Problem weiter verkompliziert. Die Herleitung der Intensitäten erfolgt daher im Appendix. In der Theorie werden nur die beiden Endresultate für die unverzwillingte Struktur dargestellt. Hierbei wird die verkürzte Portonotation verwendet, die sich in vielen Veröffentlichungen durchgesetzt hat. Die Konfigurationen werden mit zwei Buchstaben angegeben. Diese Buchstaben sind die jeweiligen Achsen X, X', Y und Y'. Der erste Buchstabe gibt hierbei die Polarisationsrichtung des Lasers an und der zweite Buchstabe die Richtung des Analyzators. Die Polarisationsrichtungen sind in Abb. 16 gezeigt. 


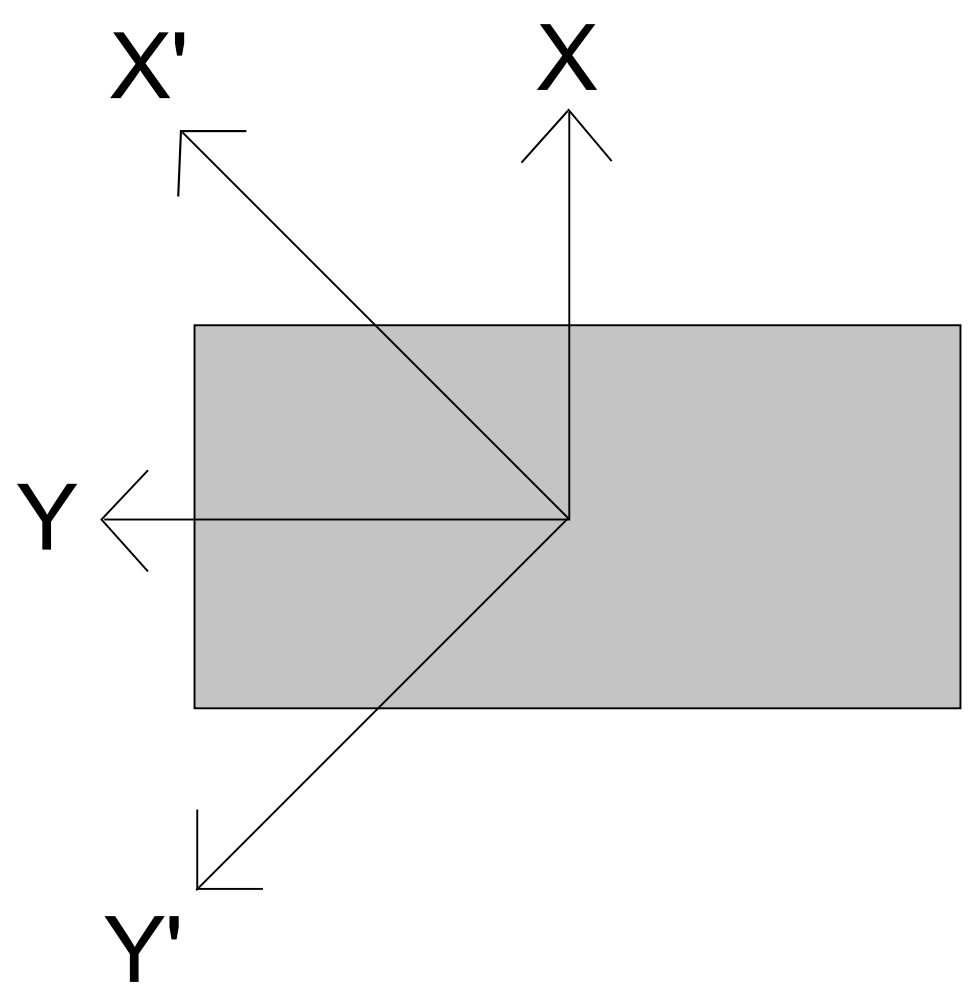

Abbildung 16 - Die Probe von oben gesehen und die vier möglichen Polarisationsrichtungen 
Die Intensitätsverhältnisse für die xy-Ebene lauten damit:

\begin{tabular}{|c|c|c|c|c|c|}
\hline Mode & XX & YY & XY & $\mathrm{X}^{\prime} X^{\prime}$ & $\mathrm{X}^{\prime} Y^{\prime}$ \\
\hline$A_{g}$ & $a^{2}$ & $b^{2}$ & 0 & $\frac{1}{4}(a+b)^{2}$ & $\frac{1}{4}(a-b)^{2}$ \\
\hline$B_{1 g}$ & 0 & 0 & $d^{2}$ & $d^{2}$ & 0 \\
\hline$B_{2 g}$ & 0 & 0 & 0 & 0 & 0 \\
\hline$B_{3 g}$ & 0 & 0 & 0 & 0 & 0 \\
\hline
\end{tabular}

Für die Proben die nicht entlang der c-Achse aufwachsen sondern entlang (011) ist es außerdem nötig noch eine Drehung um den Eulerwinkel $\Psi=45^{\circ}$ zu berechnen. Die Intensitätsverhältnisse ergeben sich damit zu:

\begin{tabular}{|c|c|c|c|c|c|}
\hline Mode & XX & YY & XY & $\mathrm{X}^{\prime} \mathrm{X}^{\prime}$ & $\mathrm{X}^{\prime} \mathrm{Y}^{\prime}$ \\
\hline$A_{g}$ & $\frac{1}{4}(a+c)^{2}$ & $b^{2}$ & 0 & $\frac{1}{4}(a+2 b+c)^{2}$ & $\frac{1}{4}(2 b-a-c)^{2}$ \\
\hline$B_{1 g}$ & $d^{2}$ & $d^{2}$ & 0 & $2 d^{2}$ & 0 \\
\hline$B_{2 g}$ & 0 & 0 & 0 & $\frac{1}{4} e^{2}$ & $\frac{1}{4} e^{2}$ \\
\hline$B_{3 g}$ & 0 & 0 & 0 & $2 f^{2}$ & 0 \\
\hline
\end{tabular}

Des Weiteren ist es möglich die Ramanmessungen temperaturabhängig durchzuführen. Mit der Hilfe von Sebastian Merten wurde der optische Kryostat, Microstat MO von Oxford, mit dem Ramanspektrometer gekoppelt um temperatur- und magnetfeldabhängige Messungen des Ramanspektrums durchzuführen. Der Kryostat kann dabei bei Temperaturen $\mathrm{T}=6-300 \mathrm{~K}$ und Magnetfeldern $\mathrm{B}=0-5 \mathrm{~T}$ arbeiten. Die Ramanlinien zeigen eine erhöhte Intensität und verringerte Halbwertsbreite sowie eine Verschiebung zu höheren Wellenzahlen unter Verringerung der Temperatur. Die Ursache hierfür ist in der quantenmechanischen Beschreibung des Ramanprozesses sowie dem anharmonischen Potentialmodell zu suchen. Die Temperaturausdehnung des Gitters bewirkt ebenfalls eine Verschiebung der Ramanlinien. Die Probe kann im Kryostaten nur nach erneutem Aufheizen gedreht werden. Dadurch benötigt eine komplette Charakterisierung aller vier Polarisationen viel Zeit und hysteretische Effekte können schwieriger untersucht werden. 


\subsubsection{Tip enhanced Raman spectroscopy}

TERS oder Tip Enhanced Raman Spectroscopy ist eine Technik, bei der eine vergoldete STM oder AFM Spitze an eine Probe angenähert wird und die plasmonischen Resonanzen der Goldspitze dazu verwendet werden die Intensität des Ramansignals zu erhöhen. Dieses Verfahren, das sich aus der Idee von SERS (Surface enhanced raman spectroscopy) entwickelt hat, wurde anfänglich hauptsächlich für biologische Proben eingesetzt, da diese typischerweise ein sehr schwaches Ramansignal zeigen und man die Laserintensität, die ebenfalls die Intensität der Moden beeinflussen kann, nur geringfügig erhöhen kann bevor die Probe verbrennt. Um dieses Problem zu umgehen, wurde zuerst SERS und später TERS benutzt. In letzter Zeit wird TERS aber immer mehr auch für Oxide oder kristalline Materialien verwendet, da es auch hier vorkommen kann, dass die Ramanmoden, aufgrund der Kristallsymmetrie oder der Polarisierbarkeit der einzelnen Bestandteile, ein sehr geringes Ramansignal zeigen [60,61. Bei der Benutzung des TERS muss darauf geachtet werden, dass für die ursprüngliche Kalibrierung des Setups aufgrund der seitlichen Einstrahlung weniger Intensität zur Verfügung steht. Die angenäherte Goldspitze erzeugt ein sogenanntes Depolarisierungsfeld wodurch polarisationsabhängige Messungen mit dem TERS komplizierter sind als bei einfachen Ramanmessungen. 


\section{Ergebnisse}

\subsection{Evolution der Probenherstellung}

Die Probenherstellung, die mit der MAD geschah, hat während der Arbeit verschiedene Anpassungsschritte erfahren, die hier im Weiteren erläutert werden sollen. Die Idee hochdotierte LCMO Proben herzustellen, entsprang dem Bedarf von einkristallinen Proben für THz-Messungen. Bei Messungen an Polykristallen ist in vorangegangenen Messungen eine Überstruktur für verschiedene kommensurable Dotierungen gefunden worden. Ein Ziel war daher zu überprüfen, ob diese Überstrukturpeaks auch in einkristallinen Proben auftreten.

Hierfür wurden 100nm dicke Schichten in der MAD1 auf MgO hergestellt, die in einem ersten Test in Stuttgart vermessen wurden. Hierbei stellte sich heraus, dass die Proben zwar für das THz-Spektrometer, nicht jedoch für das FIR-Spektrometer geeignet sind. Der Grund hierfür ist das MgO Substrat, das starke Absorptionslinien im fernen Infrarot zeigt [64 und somit das Signal der Probe drastisch verfälscht. Diese Tatsache führte dann zu der Erkenntnis, dass 1. möglichst dicke Proben nötig sind um auch schon für den THz-Bereich ein möglichst großes Signal der Probe zu erhalten, das nicht durch das $\mathrm{MgO}$ verfälscht ist, sowie 2. die Notwendigkeit freistehende Schichten herzustellen.

Ein Substrat zu verwenden das nicht infrarotaktiv ist, erwies sich als nicht praktikabel, da alle Substrate die eine passende Gitterkonstante aufweisen Infrarotbanden in der Nähe von $\mathrm{MgO}$ zeigen. Die oxidischen Substrate, deren Gitterparameter gut mit denen von LCMO übereinstimmen, kennzeichnen sich alle durch Infrarotaktivität |65,66|. Wobei sich durch die Gitterkonstante die Position der Absorptionsbanden verschieben lässt. Die Banden sind aber so breit, dass es nicht möglich ist einfach nur viele Schichten auf unterschiedlichen Substraten herzustellen und so Stück für Stück das gesamte Spektrum zu messen, immer die Lücken zwischen den Absorptionsbanden ausnutzend.

Eine weiterführende Überlegung war es Substrate zu finden, die von Lauge angegriffen und schlussendlich komplett aufgelöst werden konnten, da die Manganatschichten, auch bei tagelanger Lagerung in drei molarer Natronlauge, keine Veränderung ihrer Eigenschaften zeigten, wie selbst durchgeführte XRD und Widerstandsmessungen zeigten. Alle Versuche ein solches Substrat $\mathrm{zu}$ finden waren vorerst nicht erfolgreich. Stattdessen sollten nun die Beschränkungen des Depositionsverfahrens bezüglich der maximalen Dicke der Proben ausgelotet werden. Neuere Proben mit einer Dicke von über 200nm zeigten weiterhin die schon bekannten Eigenschaften, woraufhin die Dicke ein 
weiteres Mal erhöht wurde.

Im Laufe dieses Prozesses wurde allerdings offensichtlich, dass die Proben ab einer gewissen Dicke einen rapiden Qualitätsverlust erleiden wahrscheinlich bedingt durch die Änderung der Präkursorlösung mit der Standzeit vor der Deposition. Die Lösung zeigt nach längerer Zeit eine Farbänderung. Diesem Prozess kann durch zeitlich abgestimmte Präparation von mehreren Präkursorlösungen entgegengewirkt werden.

Als Endergebnis wurde eine Dicke von 530nm als neuer Standard für die dicken LCMO Schichten verwendet, die in der MAD1 hergestellt wurden und alle weiteren Schichten mit dieser Dicke hergestellt. Die Depositionsparameter in der MAD1 sind hierbei: Temperatur, Geschwindigkeit der peristaltischen Pumpen, La/Ca- und $\mathrm{La}+\mathrm{Ca} / \mathrm{Mn}$-Verhältnis sowie Molarität.

Die mit dem Pyrometer gemessene Temperatur lag bei $\mathrm{T}=1060-1120^{\circ} \mathrm{C}$ bei einer Heizerspannung von $\mathrm{U}=30-31 \mathrm{~V}$. Die verwendete Geschwindigkeit der Pumpen betrug 0,5 $\mathrm{ml}$ pro Minute. Das La/Ca Verhältnis betrug 1 und das $\mathrm{La}+\mathrm{Ca} / \mathrm{Mn}$ Verhältnis 1,3326. Die verwendete Molarität war 0,022mol/l.

In den 530nm dicken Schichten kann Ladungsordnung in den Widerstandskurven beobachtet werden, obwohl die Ausprägung immer noch geringer ausfällt als in Bulkmaterialien derselben Dotierung. Nachdem Schichten von guter Qualität hergestellt werden konnten, war die prävalente Aufgabe wieder freistehende Filme herzustellen. Die freistehenden Filme wurden mit einem Verfahren hergestellt, dass sich an Banerjee [67| anlehnt und von Markus Michelmann weiterentwickelt wurde. Dabei wird eine Lage von $\mathrm{ZnO}$ zwischen zwei Oxiden benutzt um Filme zu strukturieren. Das $\mathrm{ZnO}$ ist anders als viele andere Oxide anfällig gegen basische Lösungen und kann daher selektiv geätzt werden. Bei Banerjee wurde zu diesem Zweck $\mathrm{AlO}$ verwendet, bei der Deposition mit der MAD ist dieses Material jedoch nicht anwendbar, da es sich bei den Depositionstemperaturen der MAD zu Saphir umwandelt, das nicht selektiv geätzt werden kann. Markus Michelmann hatte deshalb die Idee $\mathrm{ZnO} z u$ verwenden, das auch bei hohen Temperaturen immer in derselben Phase vorhanden ist. Damit war es möglich selektiv das $\mathrm{ZnO}$ zu ätzen und eine Strukturierung der Filme zu ermöglichen. Dafür werden die Proben in Natronlauge gelegt und nach einigen Stunden aus der Lösung genommen. Das Manganat, das einen pH-Wert von 13 hat, ist dabei vollkommen inert und zeigt keinerlei Veränderung der Eigenschaften der Probe.

In dieser Arbeit wurde nun dieser Strukturierungsprozess zum ersten Mal verwendet um freistehende Filme herzustellen. Die Filme wurden dabei typischerweise auf $\mathrm{Al}_{2} \mathrm{O}_{3}$ hergestellt. Auf dem Saphirsubstrat wurde eine dünne Lage $\mathrm{MgO}$ deponiert, das Verspannungen des $\mathrm{ZnO}$ Films reduziert und die Oberfläche glättet 68]. Auf dem $\mathrm{MgO}$ wurden nun ungefähr $100 \mathrm{~nm} \mathrm{ZnO}$ deponiert. Es ist hierbei wichtig, dass die ZnO Schicht eine Dicke von über 
60nm hat, da der Ätzprozess ansonsten keinerlei Einfluss auf die Proben zeigt. Auf dem $\mathrm{ZnO}$ wurde dann eine weitere Schicht $\mathrm{MgO}$ deponiert, die eine Orientierung in [111]-Richtung zeigt und somit vom hexagonalen $\mathrm{ZnO}$ ein Wachstum auf einer kubischen Schicht ermöglichen soll |69|. Auf der MgO Schicht wurde dann letztendlich der LCMO-Film deponiert. Es wurden hierbei dieselben Depositionsparameter für die Manganatschichten verwendet wie bei den Schichten auf MgO. Die ZnO- und MgO-Schichten wurden bei Temperaturen $T \approx 650^{\circ} \mathrm{C}$ hergestellt bei einer Heizerspannung von $\mathrm{U}=19,7 \mathrm{~V}$. Die Lösung mit Zinkacetat wieß eine Molarität von $0,075 \mathrm{~mol} / \mathrm{l}$ auf und die Lösung für Magnesiumacetat von $0,106 \mathrm{~mol} / \mathrm{l}$. Beide Schichten wurden mit der maximalen Geschwindigkeit von 3,3 ml pro Minute hergestellt.

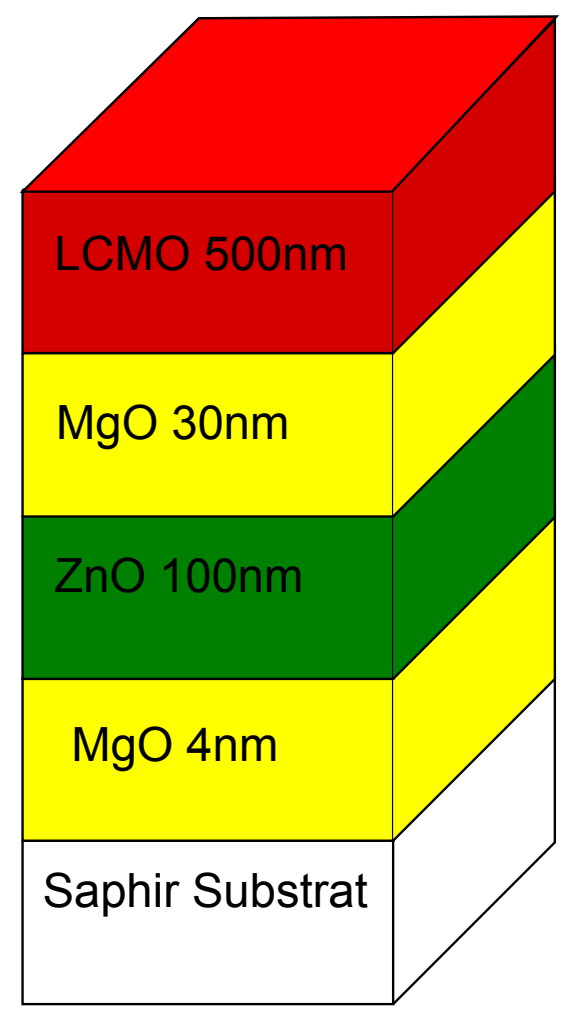

Abbildung 17 - Layerstack der freistehenden Filme. Die Layerdicken sind nicht proportional zur wirklichen Dicke gezeichnet

Nach der Deposition müssen die Seiten des Substrats mit einem Schmirgelpapier vorsichtig abgeschliffen werden, um Manganat, das möglicherweise an der ZnO-Schicht vorbeigewachsen ist, zu entfernen. Nach Abspaltung der seitlichen Substratteile, die während der Deposition von der Klammer bedeckt waren, werden die Filme weiter bearbeitet. Die Strukturen werden für 
einige Tage in eine drei molare $\mathrm{NaOH}$ Lösung gelegt. Abhängig von der Dotierung lösen sich die Filme gut oder eher zögernd vom Substrat. Für einen Film mit Dotierung $\mathrm{x}=0,5$ konnte beobachtet werden, wie der Film sich im Ganzen abgelöst hatte und auf der Lösung aufschwamm. Die Filme sind aufgrund der geringen Dicke sehr anfällig für jegliche äußeren Kräfte und zerbrechen bei der kleinsten Krafteinwirkung in winzig kleine Teile. Wenn man einen Tesafilm auf die Filme klebt, bevor man sie in $\mathrm{NaOH}$ auslagert, kann man damit gewährleisten, dass die Filme nicht zerbrechen. Zieht man nach dem Bad in NaOH den Tesafilm vorsichtig ab, so bleibt der Film daran hängen. Leider erhält der Film bei dieser Behandlung typischerweise viele Mikrorisse, da das Tesaband elongiert wird. Dies kann nur verhindert werden, wenn sich der gesamte Film gelöst hat und keinerlei Krafteinwirkung nötig ist um den Tesa zu entfernen. Allerdings reichen selbst unter diesen Bedingungen kleinste Krafteinwirkungen um Mikrorisse zu erzeugen. Dies ist auch der Grund, warum die freistehenden Filme nicht mit dem PPMS vermessen werden konnten, da kein zusammenhängender Film vorhanden ist. Für die THz-Messungen spielen solcherlei Mikrorisse für die Auswertung laut Boris Gorshunov allerdings keine Rolle.

Als weitere Verbesserung der kristallinen Qualität wurden ebenfalls Schichten auf gekauftem ZnO [0001] Substraten hergestellt. Die Substrate weisen durch den Poliervorgang geringere Oberflächenrauigkeiten auf als die von uns hergestellten Multilagen. Auf den $\mathrm{ZnO}$ Substraten wurde dann mit einem $\mathrm{MgO}$ Buffer die eigentliche LCMO Schicht deponiert. Die dabei entstandenen Proben sind qualitativ hochwertiger als das vorher vorgestellte Prinzip der Multilagen. Ein Nachteil dieses Verfahrens ist jedoch, dass die Proben bei dem Strukturierungsprozess komplett zerstört werden, da der LCMO Film in extrem kleinen Stücken vom Substrat abplatzt. Da nur wenige dieser Proben hergestellt wurden, wurde nicht versucht auch auf diese Filme einen Tesafilm zu kleben. Auch die Schichten auf $\mathrm{ZnO}$ zeigen bei den $\Phi$-Scans eine sechszählige Symmetrie, wie im Weiteren auch für die freistehenden Filme auf Saphir gezeigt wird. Die in dieser Arbeit hergestellten freistehenden Filme sind also verzwillingte Kristalle. Die kristalline Qualität der freistehenden Kristalle ist damit trotzdem besser als die weithin verwendeten Keramiken, die typischerweise weitere Kristallorientierungen enthalten. Dies wird auch deutlich an den THz-Messungen der freistehenden Filme. Mehr dazu im Kapitel THz-Spektroskopie. In Stuttgart gelang es Gabi Köster die Schichten im Ganzen von den Substraten zu lösen. Boris Gorshunov und Elena Zhukova führten daraufhin die allerersten Infrarotaufnahmen von freistehenden Manganatschichten durch. Die Proben sind zum jetzigen Zeitpunkt, nach Kenntnis des Autors, die einzigen vollständig freistehenden Manganatfilme die jemals hergestellt wurden. Die Technik die hier verwendet wurde lässt 
sich dabei auch auf andere Oxidsysteme anwenden.

In einem letzten Schritt wurde nach den FIR-Messungen in Stuttgart eine Dotierungsreihe auf $\mathrm{MgO}$ mit der MAD3 hergestellt. Der Vorteil der MAD3 liegt in den verwendeten Spritzendosierern und der ebenfalls verwendeten Ellipsometriemessung. Bei der Herstellung mit der MAD3 wurde die Schichtdicke leicht auf $425 \mathrm{~nm}$ verringert. Mit Ausnahme des $\Phi$-Scans sind alle im Weiteren präsentierten Messungen, von Filmen auf $\mathrm{MgO}$, mit der MAD3 hergestellt worden. Filme die mit der MAD1 hergestellt wurden werden gesondert erwähnt. Die Proben die mit der MAD3 hergestellt wurden sind aufgrund des unterschiedlichen Heizers bei leicht geringeren Temperaturen hergestellt worden. Dies hat Einfluss auf das Wachstum der Schichten mit Dotierungen $x \geq 0,85$, wie im Folgenden ausgeführt wird.

\subsection{Struktur von LCMO und verwendete Proben}

Die Probencharakterisierung gestaltet sich im überdotierten LCMO System als deutlich schwieriger, als für die konventionell verwendeten LSMO Systeme. Der überdotierte Bereich, der schon generell weit weniger erforscht ist als der niedrig dotierte um $x \approx 0,33$, weist als erschwerendes Kriterium die Probenqualität auf. Überdotiertes LCMO kann besonders im ladungsgeordneten Bereich nicht als Einkristall hergestellt werden. Damit ist die Qualität der Proben auf Polykristalle und Keramiken beschränkt. In einem solchen Fall stellen epitaktische Filme eine attraktive Quelle für hochreine Kristalle dar. Allerdings stellen sich bei Dünnfilmen andere Probleme. Ein bekanntes Problem ist die Gitterverzerrung. Dünne Filme können einkristallin aber verspannt auf verschiedensten Substraten aufwachsen. Hierbei spielt die Gitterkonstante des darunterliegenden Substrats die entscheidende Rolle, sowie die Orientierung in welcher der Film aufwächst. Typischerweise zielt man darauf ab, dass die Länge der Achsen des Substrats möglichst gut mit der Länge der Kristallachsen des Films übereinstimmt, sodass der Film in [001] Richtung epitaktisch aufwächst. Wenn die Gitterkonstanten nicht exakt zueinander passen, wachsen die Filme bis zu einer gewissen Dicke verspannt auf, die typischerweise von dem Grad der Verspannung abhängt und zeigen dann Verzwillingung oder andere Defekte, um die immer größer werdende freie Energie der Verspannung abzubauen. Um diese Effekte zu untersuchen wurden verschiedene Charakterisierungsmethoden angewandt, die insgesamt genommen ein möglichst vollständiges Bild des Systems liefern sollen. In Tabelle 1 sind außerdem alle verwendeten Proben aufgelistet. 


\begin{tabular}{|c|c|c|c|}
\hline Name & Dotierung $\mathrm{x}$ & Dicke $[\mathrm{nm}]$ & verwendet für \\
\hline H519 & $\mathrm{x}=0,8$ & 530 & Bilder der freistehenden Filme \\
\hline $\mathrm{H} 522$ & $\mathrm{x}=0,66$ & 530 & Bilder der freistehenden Filme \\
\hline $\mathrm{H} 524$ & $\mathrm{x}=0,5$ & 530 & Bilder der freistehenden Filme \\
\hline H536 & $\mathrm{x}=0,5$ & 530 & Dotierung $\mathrm{I}(100) / \mathrm{I}(200)$ \\
\hline H539 & $\mathrm{x}=0,85$ & 530 & Dotierung $\mathrm{I}(100) / \mathrm{I}(200)$ \\
\hline H540 & $\mathrm{x}=0,96$ & 530 & Dotierung $\mathrm{I}(100) / \mathrm{I}(200)$ \\
\hline $\mathrm{X} 810$ & $\mathrm{x}=0,33$ & 100 & Dotierung $\mathrm{I}(100) / \mathrm{I}(200)$ \\
\hline $\mathrm{X} 878$ & $\mathrm{x}=0,75$ & 425 & TEM Aufnahmen \\
\hline $\mathrm{X} 877$ & $\mathrm{x}=0,75$ & 425 & Dotierungsserie für alle gezeigten Messungen \\
\hline $\mathrm{X} 896$ & $\mathrm{x}=0,55$ & 425 & Dotierungsserie für alle gezeigten Messungen \\
\hline $\mathrm{X} 897$ & $\mathrm{x}=0,66$ & 425 & Dotierungsserie für alle gezeigten Messungen \\
\hline $\mathrm{X} 905$ & $\mathrm{x}=0,85$ & 425 & Dotierungsserie für alle gezeigten Messungen \\
\hline $\mathrm{X} 906$ & $\mathrm{x}=0,96$ & 425 & Dotierungsserie für alle gezeigten Messungen \\
\hline
\end{tabular}

Tabelle 1 - verwendete Proben und durchgeführte Messungen

\subsection{Röntgendiffraktometrie}

In Abb. 18 sind die $\Theta-2 \Theta$ Aufnahmen der Schichten mit steigender Dotierung von $\mathrm{x}=0,55$ bis $\mathrm{x}=0,96$ gezeigt. Die Indizierung gestaltet sich aufgrund der geringen Anzahl an Peaks als sehr einfach und erfolgt für die Schichten in der simple cubic Struktur, da aufgrund des einfachen XRD Scans nicht auf eine Struktur geschlossen werden kann. Der größte Peak der außerdem $\alpha_{1}-\alpha_{2}$ aufgespalten ist, bei $2 \Theta=42,9^{\circ}$, kann dem MgO Substrat zugeordnet werden, genauso wie der Nebenpeak bei $2 \Theta=38,5^{\circ}$, als $k_{\beta}$ Peak des Substrats. Die einzigen anderen Peaks, die auftauchen, sind die Peaks des LCMO Films. Abhängig von der Dotierung befindet sich bei $2 \Theta=23,29-23,7^{\circ} \operatorname{der}(001)_{s c}$ Peak des Substrats, in Pnma als (020) zu indizieren. Bei $2 \Theta=33,3-33.92$ befindet sich der $(011)_{s c}$ Peak und bei $2 \Theta=47,4-48,4^{\circ}$ der $(002)_{s c}$ Peak. Es sind auch in der logarithmischen Auftragung keine weiteren Peaks erkennbar, was für die gute Qualität der Proben spricht. Bei größer werdender Dotierung bildet sich ein immer größerer Anteil an (011) Phase, wie man an der Intensität des Peaks erkennen kann. Soweit das bei $\mathrm{x}=0,96$ keine (001)-Phase mehr vorhanden ist. Dies ist dadurch bedingt, dass auch CMO in (011) Orientierung auf $\mathrm{MgO}$ aufwächst |70|. Die linear kleiner werdende Gitterkonstante und der immer größer werdende Unterschied der Gitterkonstante von LCMO zu MgO sorgt für immer größere Verspannungen, bis sich schließlich die Orientierung des Films ändert und er entlang der (011) Achse aufwächst. Der Grund hierfür ist vermutlich die sogenannte „do- 
main matching epitaxy", die auch schon bei dem unverspannten Wachstum von $\mathrm{La}_{0,67} \mathrm{Ca}_{0,33} \mathrm{MnO}_{3}$ auf $\mathrm{MgO}$ eine Rolle spielt. Bei der „domain matching epitaxy" müssen $n \cdot a_{M g O}=m \cdot a_{C M O}$ sein, wobei n,m natürliche Zahlen sind. Diese Bedingung ist bei $n=8$ und $m=9$ erfüllt wenn CMO entlang der (011)-Achse des Substrats aufwächst. Dieses Verhalten ändert sich bei der Herstellung der Schichten in der MAD1 bei höheren Temperaturen. Es wurden in der MAD1 hochdotierte Schichten ohne (011)-Phase hergestellt. Die höhere Temperatur scheint das Wachstum entlang der (001)-Achse zu begünstigen. Diese Schichten wurden später für die Dotierungsbestimmung über die Laue-Gleichung verwendet. Die überdotierten Filme zeigen domainmatching-epitaxy und sind damit mit Ausnahme einer dünnen, typischerweise wenige nm dicken, Lage direkt an der Grenzfläche zum Substrat als entspannt zu betrachten. Die Gitterkonstanten können somit mit denen aus anderen Arbeiten verglichen werden. Die Auftragung ist in Abb 19 zu sehen. Hierbei wurden die Ergebnisse aus der Arbeit von Pissas und Kallias [28] unter den Graphen der eigenen gemessenen Gitterkonstanten gelegt. Die Übereinstimmung der gemessenen Werte mit den Gitterkonstanten zeigt die gute Qualität der Proben. 


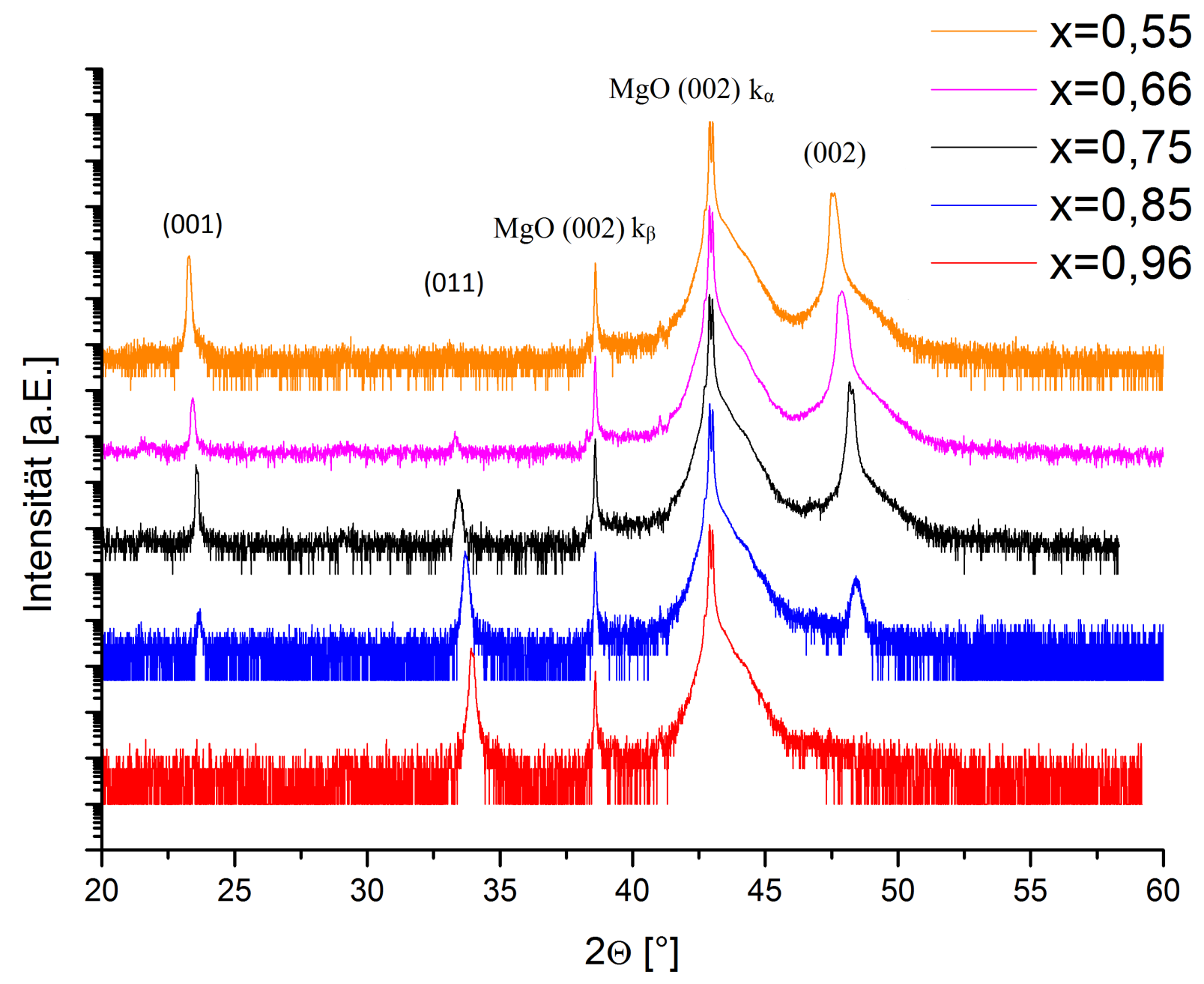

Abbildung 18 - Die Röntgenspektren der Dotierungen von $x=0,55-0,96$. Alle Peaks können LCMO-Film und MgO Substrat zugeordnet werden. Es ist keine Fremdphase vorhanden. Die Indizierung erfolgt in der simple cubic Struktur 


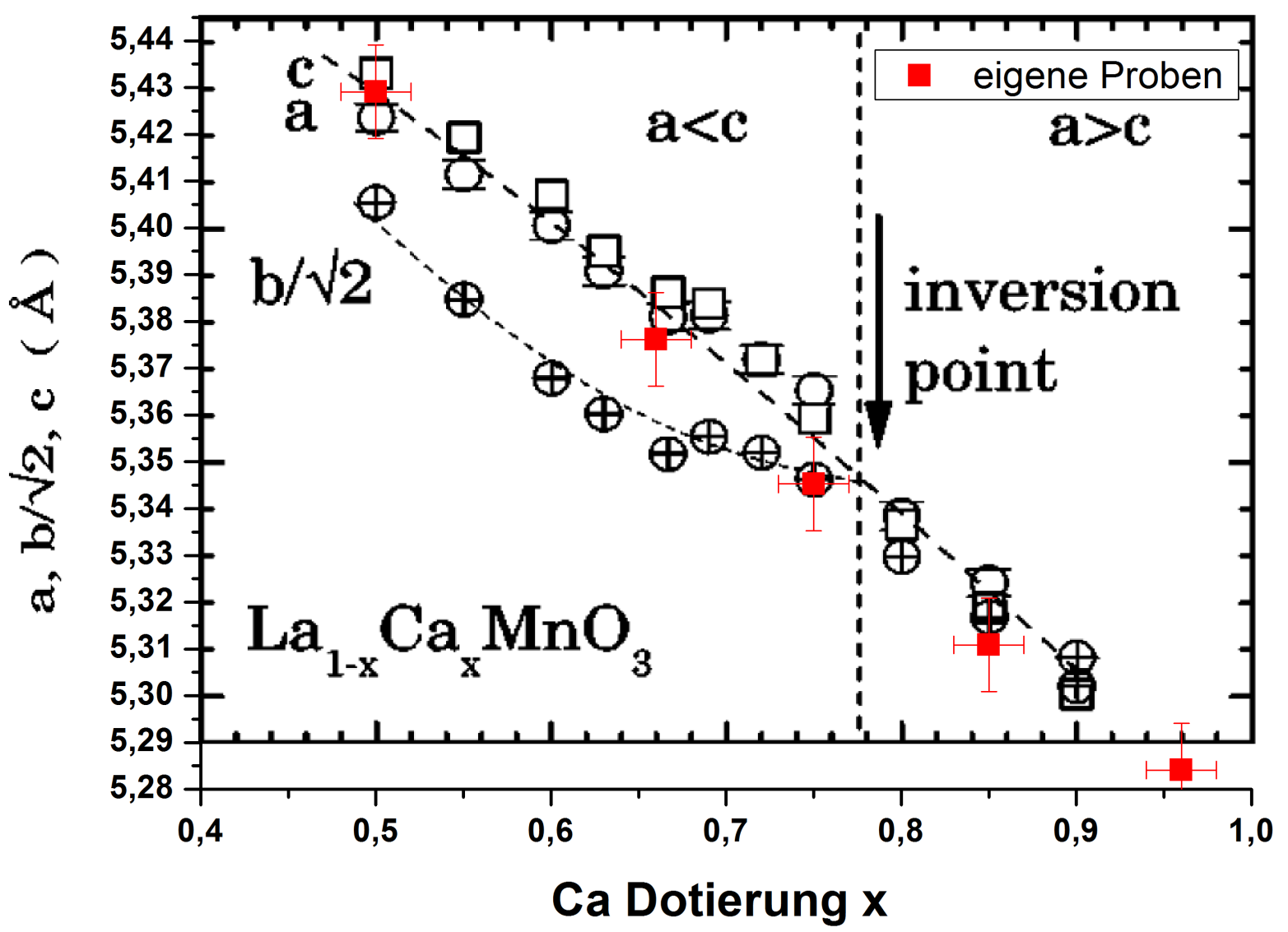

Abbildung 19 - Die Gitterkonstanten aus dem Paper von Pissas und Kallias [28] überlagert mit den Gitterkonstanten und Fehlerbalken der eigenen Filme. Die Übereinstimmung der Gitterkonstanten mit den Literaturwerten zeigt die hohe Qualität der Filme 
Eine weitere Möglichkeit die Dotierung zu verifizieren ergibt sich über den Strukturfaktor:

$\frac{I(001)}{I(002)}=\frac{\left[-(1-x) \cdot f_{L a^{3+}}-x \cdot f_{C a^{2+}}+(1-x) \cdot f_{M n^{3+}}+x \cdot f_{M n^{4+}}+f_{O^{2-}}\right]^{2}}{\left[(1-x) \cdot f_{L a^{3+}}+x \cdot f_{C a^{2+}}+(1-x) \cdot f_{M n^{3+}}+x \cdot f_{M n^{4+}}+3 \cdot f_{O^{2-}}\right]^{2}}$

Hierbei macht man sich den Umstand zunutze, dass die Intensität des $(001)_{s c^{-}}$ Peaks abhängig ist von der Ca-Dotierung. Wie bereits erwähnt werden für die Dotierung $\mathrm{x}=0,85$ und $\mathrm{x}=0,96$ zwei Proben aus der MAD1 verwendet, die deutlich größere (100) und (200) Peaks zeigen. Das Intensitätsverhältnis kann man, für die unterschiedlichen Proben, als Funktion der Calciumdotierung auftragen und mit dem theoretisch berechneten Koeffizienten, der mit den Basisvektoren der simple cubic Einheitszelle berechnet wurde, vergleichen. Dies wurde in Abb. 20 getan. Die dabei entstehende Kurve ist für jedes Material sehr spezifisch und kann zur sehr genauen Bestimmung der Konzentrationen benutzt werden.

Die Rechnung ist für die eigentliche Pnma Struktur der Proben natürlich nur eine Näherung. Nichtsdestotrotz kann man anhand der Neutronenstrukturdaten von Pissas und Kallias [28 zeigen, dass entlang der b-Achse die Pnma-Struktur nahezu der simple cubic Struktur entspricht. Die Positionen der einzelnen Atome entlang der b-Achse weichen dabei nur für eines der Sauerstoffbasisatome signifikant von der simple cubic Struktur ab. Da Sauerstoff jedoch das kleinste f besitzt ist dieser Unterschied vernachlässigbar. Die Übereinstimmung der theoretisch berechneten und experimentell ermittelten Intensitäten ist als weiteres Indiz zu werten, dass die hergestellten Proben von ausgezeichneter Qualität sind und die Berechnung der Intensitätsverhältnisse zur Bestimmung der Dotierung gerechtfertigt ist. In die Berechnung der theoretischen Kurve fließt als einziger Faktor, neben der eigentlichen LaueGleichung, nur eine Proportionalitätskonstante $k \approx 10$ ein, die die Intensität abhängig vom Winkel $2 \Theta$ skaliert. Dies ist bedingt durch eine gerätebedingte schlechtere Auflösung für höhere Streuwinkel. Die Proportionalitätskonstante wurde hierbei aus Messungen an LSMO Schichten von Markus Jungbauer bestimmt. Die Genauigkeit dieses Verfahrens ist stark von der Dicke der Schichten abhängig, da mit steigender Dicke auch die Intensitäten mit kleinerem Fehler bestimmt werden können. 


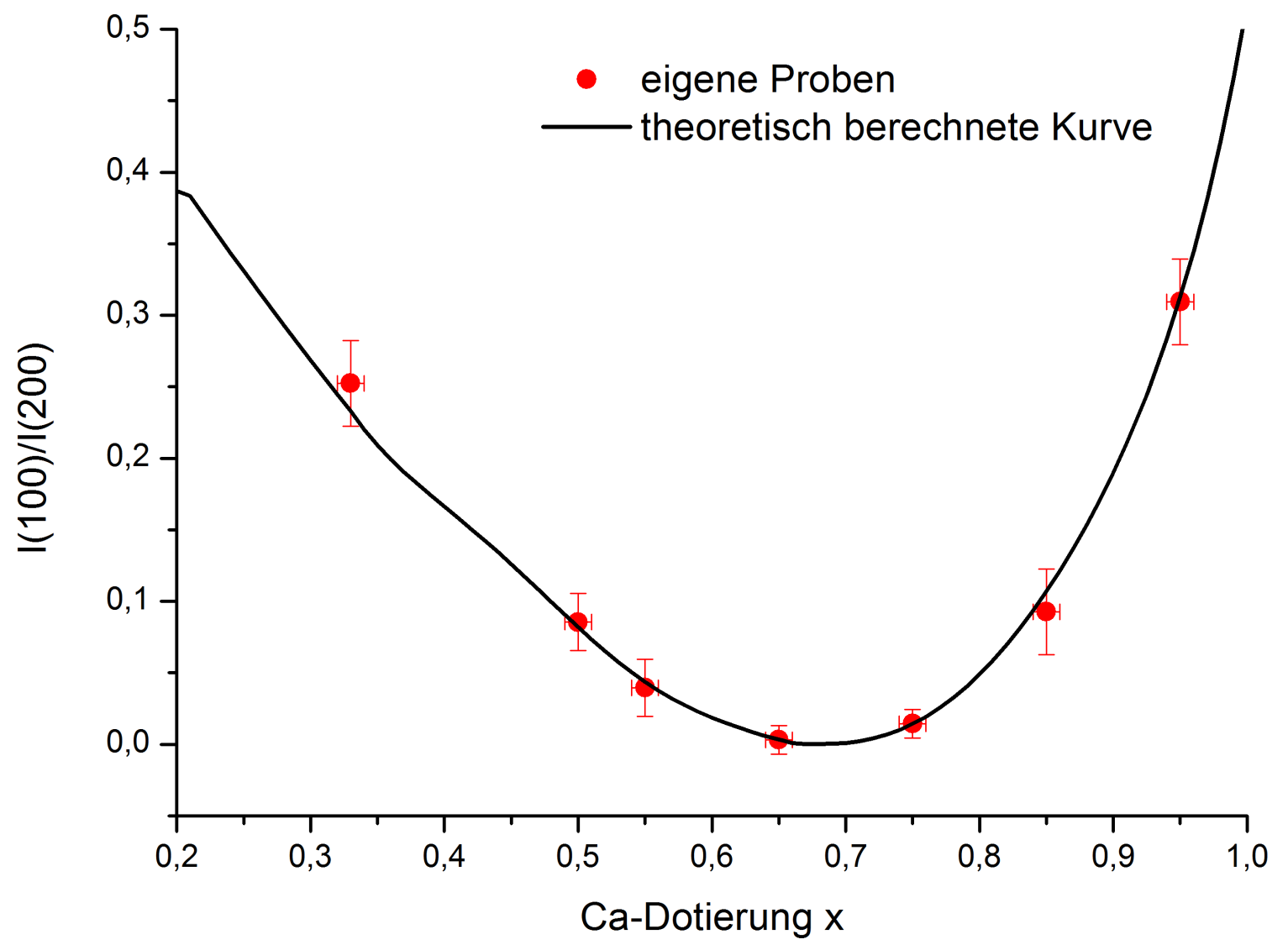

Abbildung 20 - Über den Strukturfaktor lassen sich mit gegebenen atomaren Streufaktoren die Intensitäten der einzelnen Peaks berechnen. Diese Rechnung zeigt, dass der (001) Peak für Dotierungen nahe $x=0,66$ eine Auslöschung zeigt 
Bei den $\Phi$-Scans mit der D5000 wird die Einheitszelle als kubisch angenommen und theoretisch berechnet bei welchen Werten für $\Theta$ und $2 \Theta$ der $\Phi$-Scan durchgeführt werden muss. Hierbei ist die Orientierung des Kristalls entscheidend, sowie die Maximalwerte des schwenkbaren Arms. Abhängig von der Kristallstruktur des Films weisen die Peaks eine Symmetrie auf, die Aufschluss über die Art und Weise des Wachstums der Schicht liefert. Sind die Peaks beispielsweise $60^{\circ}$ symmetrisch, bedeutet dies im Allgemeinen, dass die Schicht eine hexagonale Kristallstruktur aufweist, wohingegen $90^{\circ} \mathrm{Sym}-$ metrie auf eine kubische Struktur hindeutet. Die beiden untersuchten Ebenen sind in Abb. 21 und Abb. 26 zu sehen. Hierbei wird deutlich, dass der (013) Peak für die auf $\mathrm{MgO}$ gewachsenen Proben eine vierzählige Symmetrie zeigen sollte. Diese wird auch im $\Phi$-Scan beobachtet, siehe Abb. 22. Hierbei ist anzumerken, dass die Breite der Peaks hauptsächlich durch die Apparatur bedingt ist. Aufgrund einer fehlenden Blende ist die Halbwertsbreite der Peaks über $3^{\circ}$ groß. Der $\Phi$-Scan für die Probe auf MgO zeigt außerdem, dass die LCMO Peaks bei den gleichen Winkeln liegen, wie die Peaks des MgO. LCMO wächst also entlang der Achsen des MgO Substrats auf.

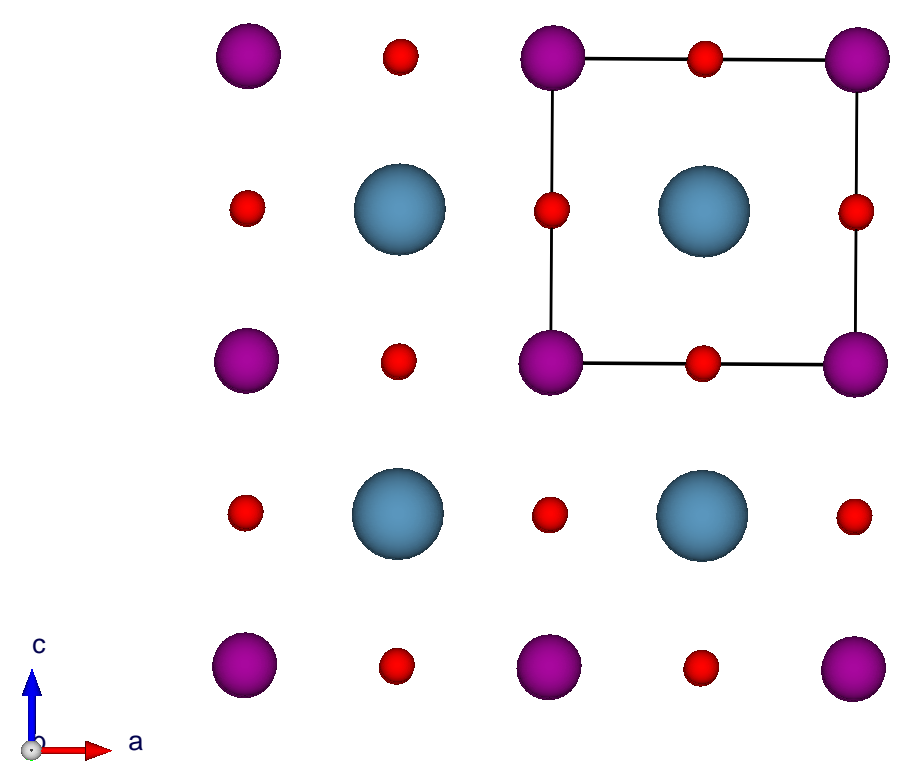

Abbildung 21 - (013) Ebene des CMO Systems 


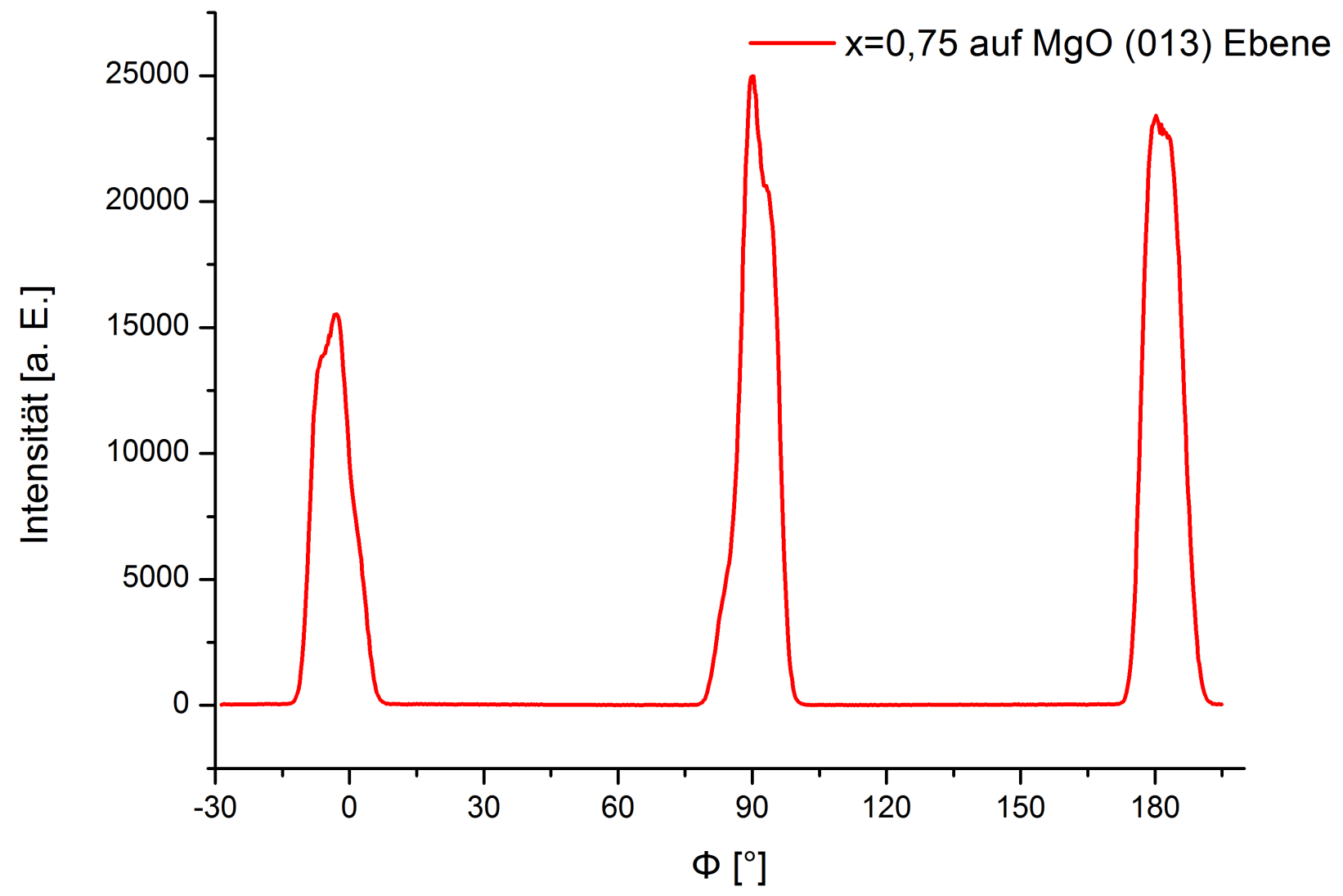

Abbildung 22 - $\Phi$-Scan eines auf $M g O$ gewachsenen LCMO Films, Dotierung $x=0,75$. Die Aufspaltung der Peaks deutet auf eine Verzwillingung hin. Leider kann aufgrund der fehlenden Blende die Mosaizität nicht bestimmt werden 


\subsection{1 freistehende Filme}

In Abb. 23 sind freistehende Filme von drei verschiedenen Dotierungen gezeigt. Der Film mit $\mathrm{x}=0,8$ zeigt dabei den kleinsten freistehenden Film. Je größer die Dotierung desto länger müssen die Filme in $\mathrm{NaOH}$ gelagert werden. Der Film mit $\mathrm{x}=0,8$ zeigt daher folgerichtig nur einen kleinen Bereich der sich ablösen ließ. Der Film mit $\mathrm{x}=0,55$ ist während der Auslagerung in $\mathrm{NaOH}$ innerhalb kürzester Zeit in kleine Stücke zerfallen. Einige von diesen Stücken haben sich vom Tesaband gelöst und konnten später mit einem Filterpapier abfiltriert werden. Die Fläche des $\mathrm{x}=0,55$ Films ist deutlich größer als beim Film mit $\mathrm{x}=0,8$. Der $\mathrm{x}=0,66$ Film zeigt nun eine nahezu perfekte Ablösung. Die äußeren Randbereiche haben sich ähnlich dem Film bei $x=0,55$ schon im Wasser vom Tesaband gelöst und sind daher auf dem Tesaband nicht zu erkennen. Der Rest des Films ließ sich hingegen ohne Probleme vom Substrat lösen. Der $\mathrm{x}=0,66$ Film ist damit der größte, in dieser Arbeit hergestellte, freistehende Film und kann aufgrund der schieren Größe für viele Messungen verwendet werden. Freistehende Manganatfilme dieser Art und Größe sind in dieser Form noch niemals hergestellt worden und stellen damit ein Novum für das Wachstum von oxidischen Schichtsystemen dar. 


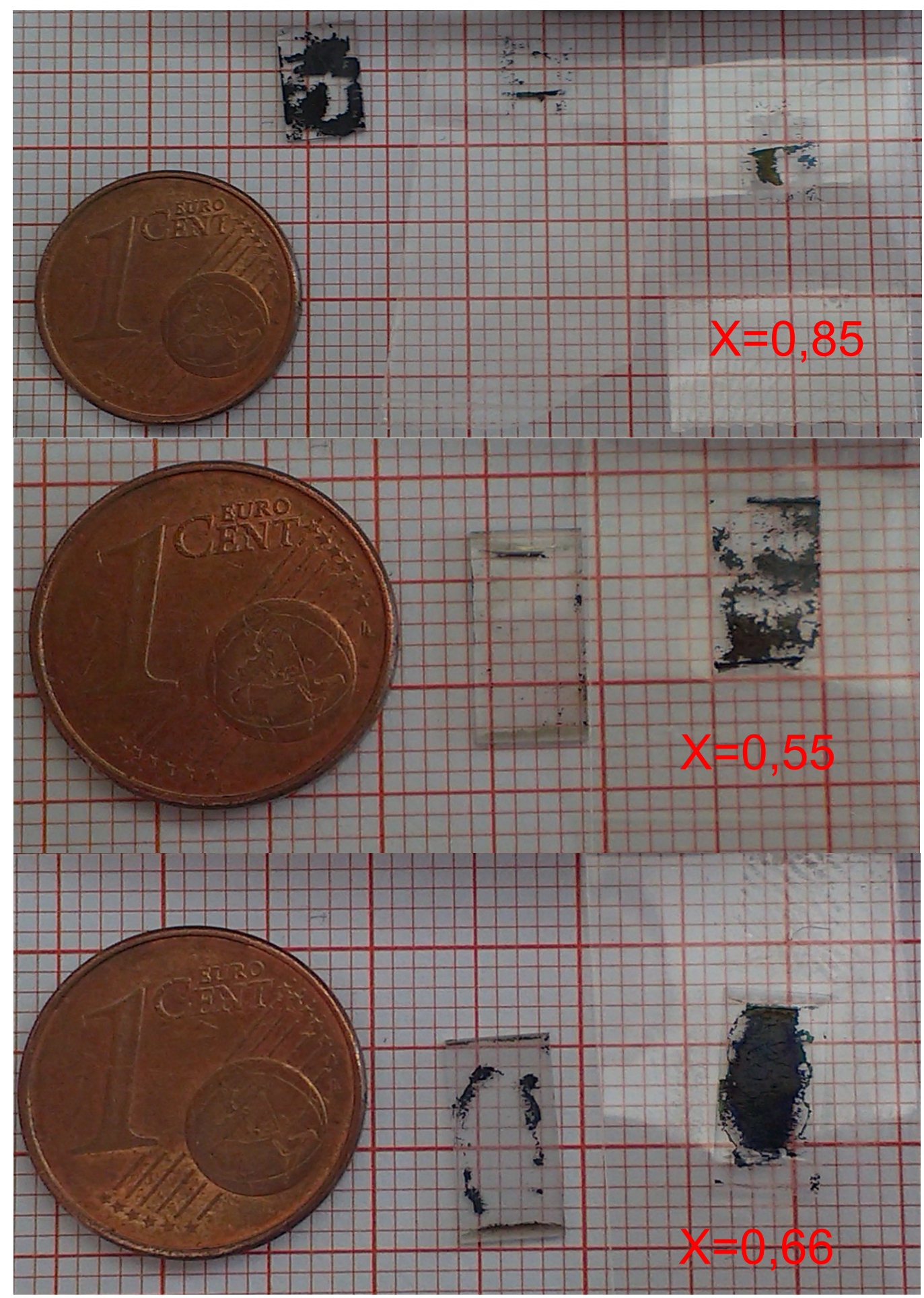

Abbildung 23 - freistehende Filme nach Auslagerung in NaOH. Auf Millimeterpapier mit einem Centstück als Maßstab 
Die Röntgenspektren der verschiedenen Dotierungen sind in Abb. 24 und Abb. 25 zu sehen.

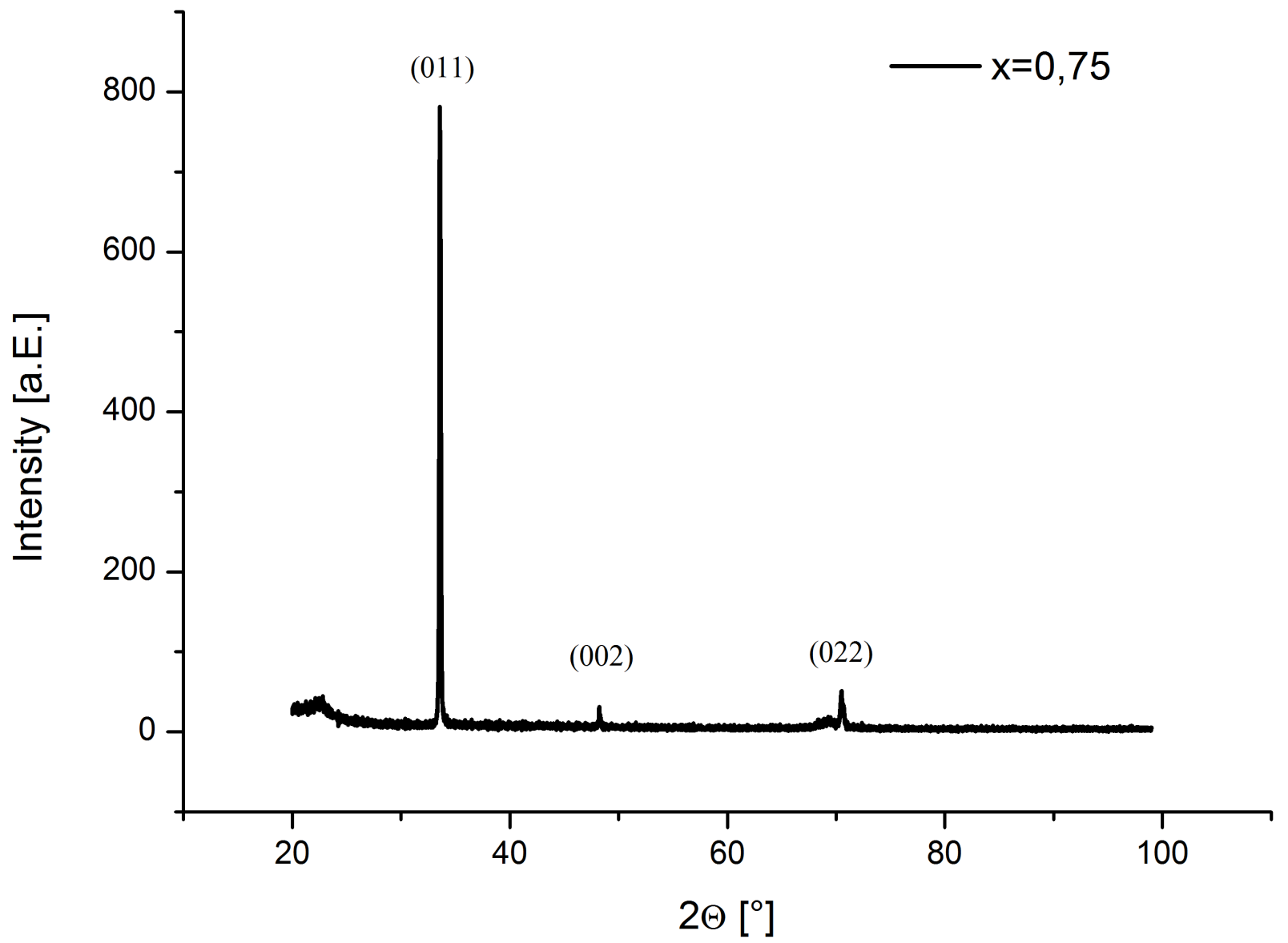

Abbildung 24 - Röntgenspektrum eines LCMO Films $x=0,75$ der nach Entfernen des Substrats auf einen Si-Wafer aufgebracht wurde. Dies geschah zur einfacheren Ausrichtung des Films in der Röntgenanlage. Der Peak des Siliziums ist in der linearen Auftragung nur schwach bei $69^{\circ} \mathrm{zu}$ sehen

Die Filme wachsen einkristallin auf und orientieren sich an dem Verhalten der Filme auf $\mathrm{MgO}$ wobei das unterliegende $\mathrm{MgO}$ die Änderung des Wachstums von hexagonal zu kubisch ermöglicht. Es wurden außerdem $\Phi$ Scans durchgeführt. Hierbei wird die Zähligkeit einer Kristallebene vermessen. So kann unterschieden werden, ob die Struktur hexagonal oder kubisch aufwächst. Die $\Phi$-Scans für die freistehenden Filme weisen hierbei auf ein einkristallines verzwillingtes Wachstum hin. Die (011)-Achse weißt in Einkristallen eine zweizählige Symmetrie auf. Durch die Verzwillingung der Pro- 


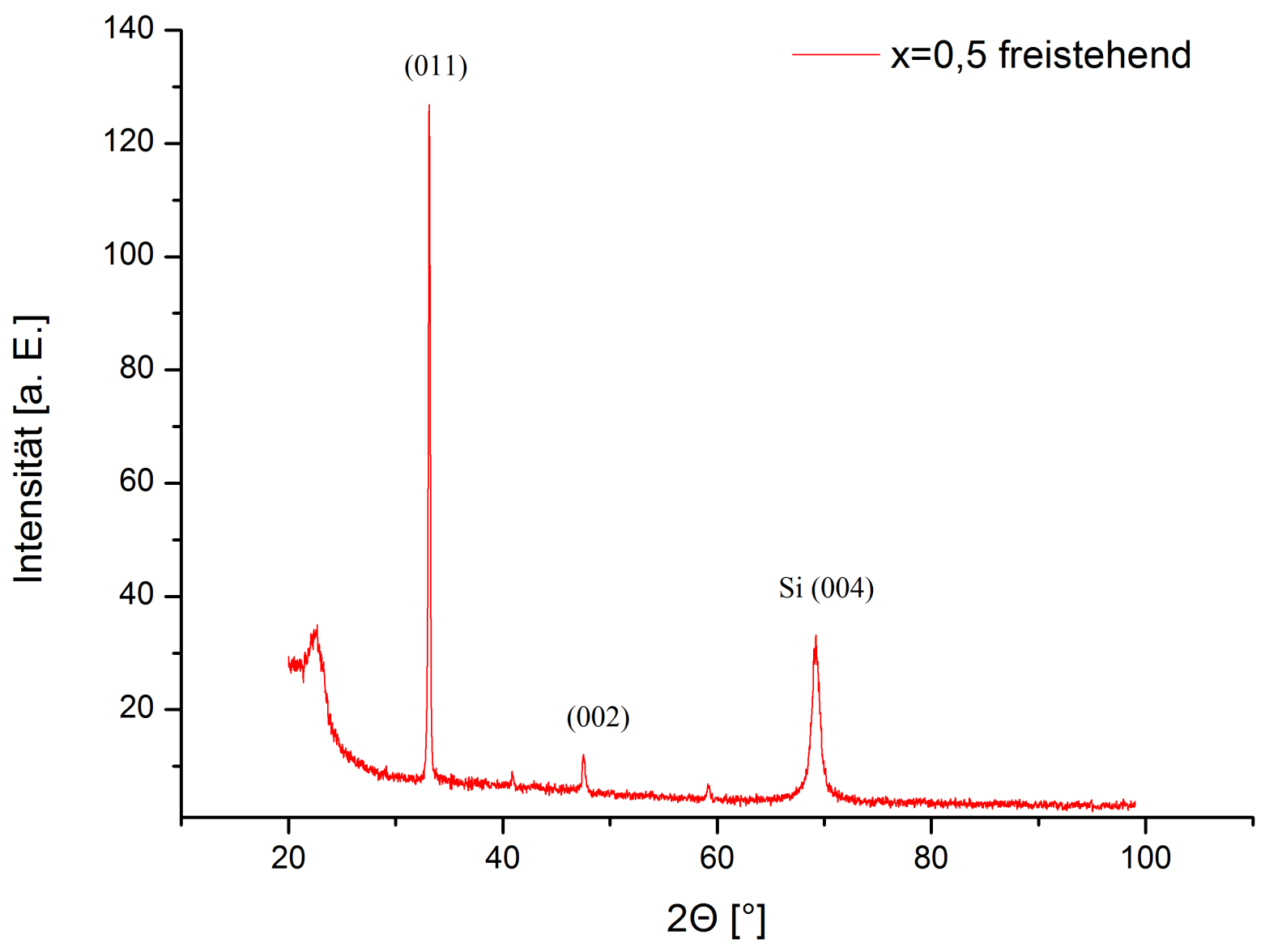

Abbildung 25 - Röntgenspektrum eines LCMO Films $x=0,5$ der nach Entfernen des Substrats auf einen Si-Wafer aufgebracht wurde.

be werden nun sechs Reflexe sichtbar. Das unterliegende $\mathrm{MgO}$ nimmt eine kubische Struktur an, da es entlang der [111] Richtung aufwächst, allerdings ist die Gitterkonstante der (111) Oberfläche zu unterschiedlich zu LCMO, sodass das LCMO in [011] Richtung aufwächst.

Es ist auch möglich, dass der Film texturiert entlang der drei Achsen, die von dem unterliegenden $\mathrm{MgO}$ (111) vorgegeben werden, aufwächst. 


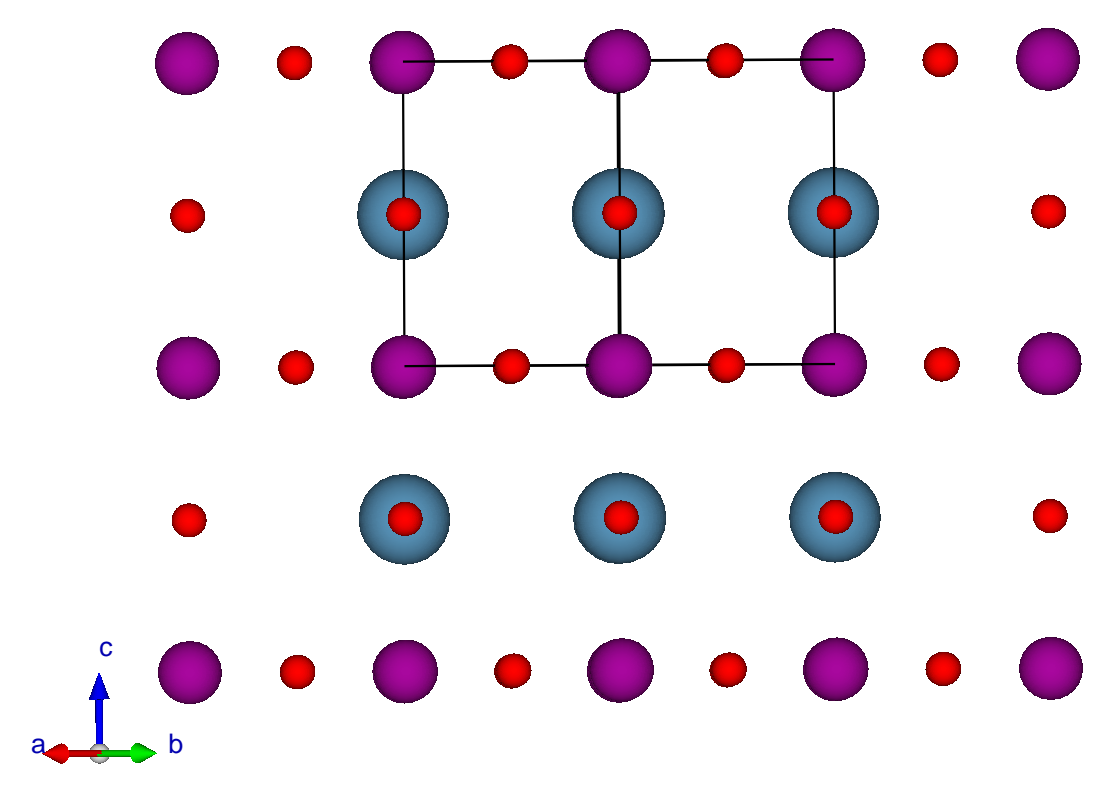

Abbildung 26 - (023) Ebene des CMO Systems 


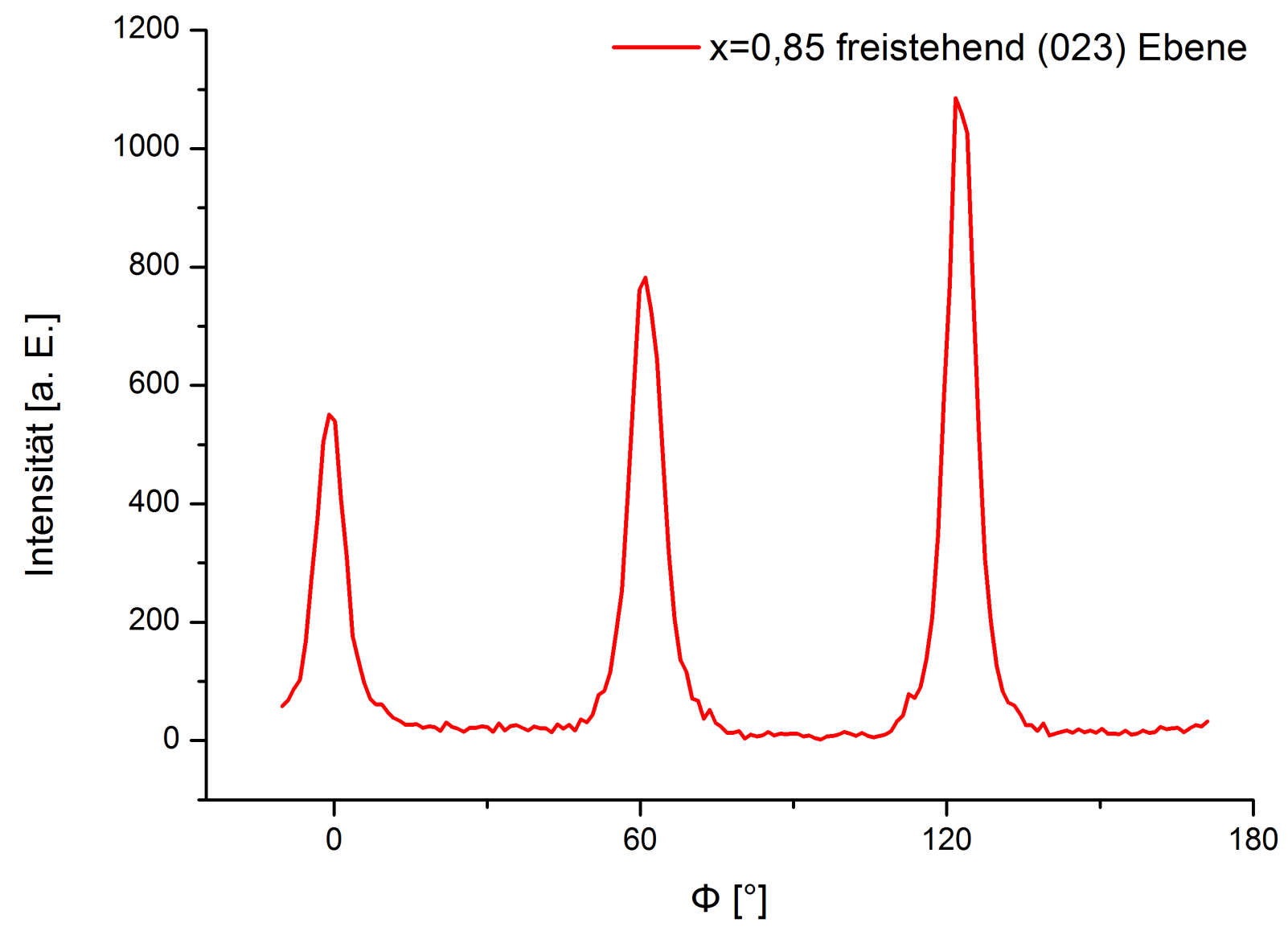

Abbildung 27 - $\Phi$-Scan des freistehenden Films mit $x=0,85$ um die (023) Ebene herum. Der Scan zeigt eine sechszählige Symmetrie 


\subsection{Lokale Struktur (TEM)}

Es wurden außerdem TEM-Aufnahmen einer Probe mit $\mathrm{x}=0,75$ von Vladimir Roddatis aus dem Institut für Materialphysik durchgeführt. Wie in Abb. 29 ersichtlich ist zeigt das HRTEM eine einkristalline Struktur sowie Verzwillingung, wie ebenfalls bestätigt durch die Ramanmessungen, für die senkrecht zum Substrat geschnittene Lamelle auf der linken Seite. Auf der rechten Seite des Bildes ist eine parallel zum Substrat geschnittene Lamelle zu sehen. Die Probe zeigt kleine Strukturen, die wie Domänengrenzen aussehen. Eine genauere Bestimmung der Zusammensetzung muss durch ein EELS Spektrum erfolgen. Die TEM Messungen demonstrieren die hohe Qualität der Schichten und zeigen den einkristallinen Charakter. Die STEM Aufnahmen, siehe Abb. 30, im HAADF zeigen mögliche kleine Schwankungen in der Verteilung von La/Ca. Der Kontrast im Bild skaliert hierbei mit $Z^{1,8} \mid 71$.

Aufgrund der TEM Messungen ist es außerdem möglich die domain-matchingepitaxy, die zuvor erwähnt wurde, zu verifizieren. Wie in Abb. 30 im Inset zu sehen ist wachsen fünf Einheitszellen LCMO auf neun Einheitszellen $\mathrm{MgO}$. Mit der Gitterkonstante von $\mathrm{MgO} a / 2=2,105 \mathrm{~nm} \mid 72]$ berechnet sich die Gitterkonstante von LCMO in der Nähe des Substrats zu $(9 \cdot 2,105 \mathrm{~nm}) / 5=$ 3,789nm. Die Gitterkonstante in der Nähe des Substrats liegt damit im Bereich von $\mathrm{x}=0,66$ dotiertem LCMO. Weiter rechts in Abb. 29 ist zu erkennen, dass sich die Anzahl der Versetzungen an der Grenze zum Substrat abhängig von der Position ändert. Für das Wachstum von $\mathrm{LCMO}$ mit $\mathrm{x}=0,33$ wurde eine ähnliche Anordnung von sechs Einheitszellen LCMO zu elf Einheitszellen $\mathrm{MgO}$ beobachtet $73 \mid$.

Die TEM Bilder zeigen auch, dass in der Probe ein Ruddelson-Popperdefekt gefunden werden konnte, gekennzeichnet durch die rote Ellipse in Abb. 30. Hier liegt vermutlich eine La/Ca Lage direkt an einer weiteren La/Ca Lage. Außer der Verzwillingung der Probe können nur wenige weitere Defekte beobachtet werden und die Probe kann als verzwillingter Einkristall angesehen werden. 

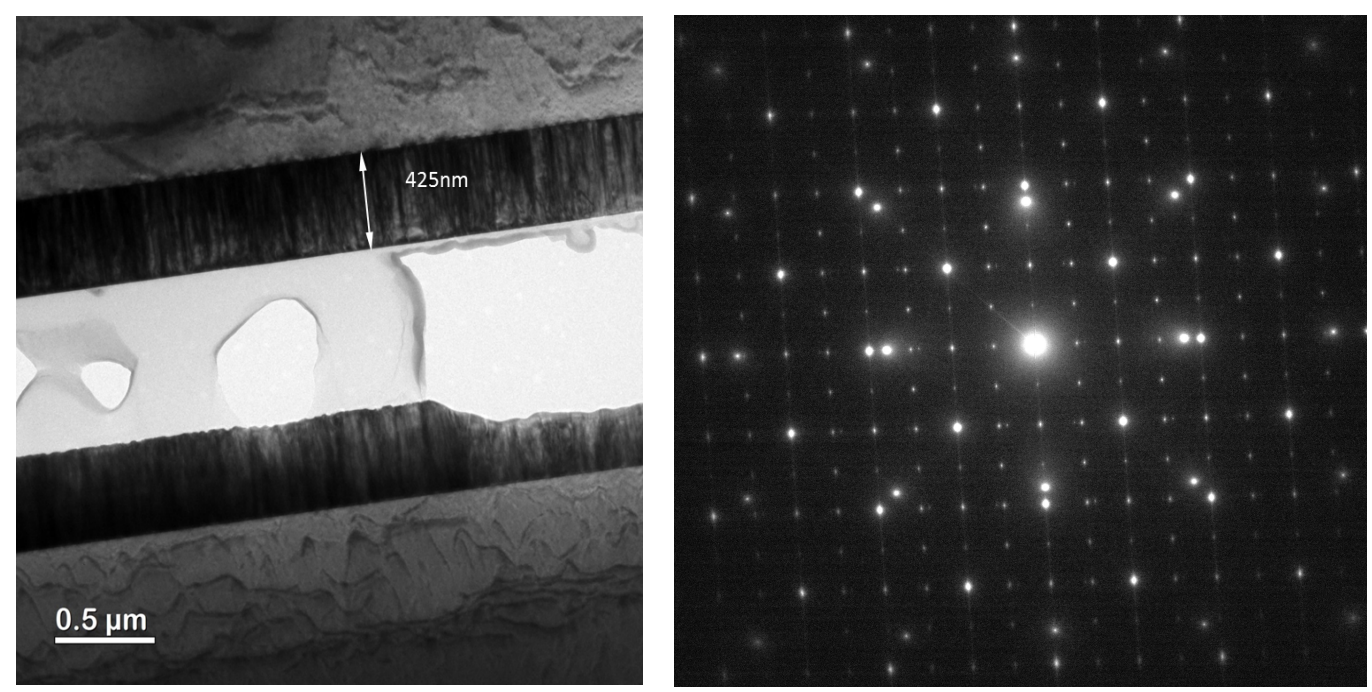

Abbildung 28 - Die präparierte TEM-Lamelle mit Verifizierung der Schichtdicke und Beugungsbild
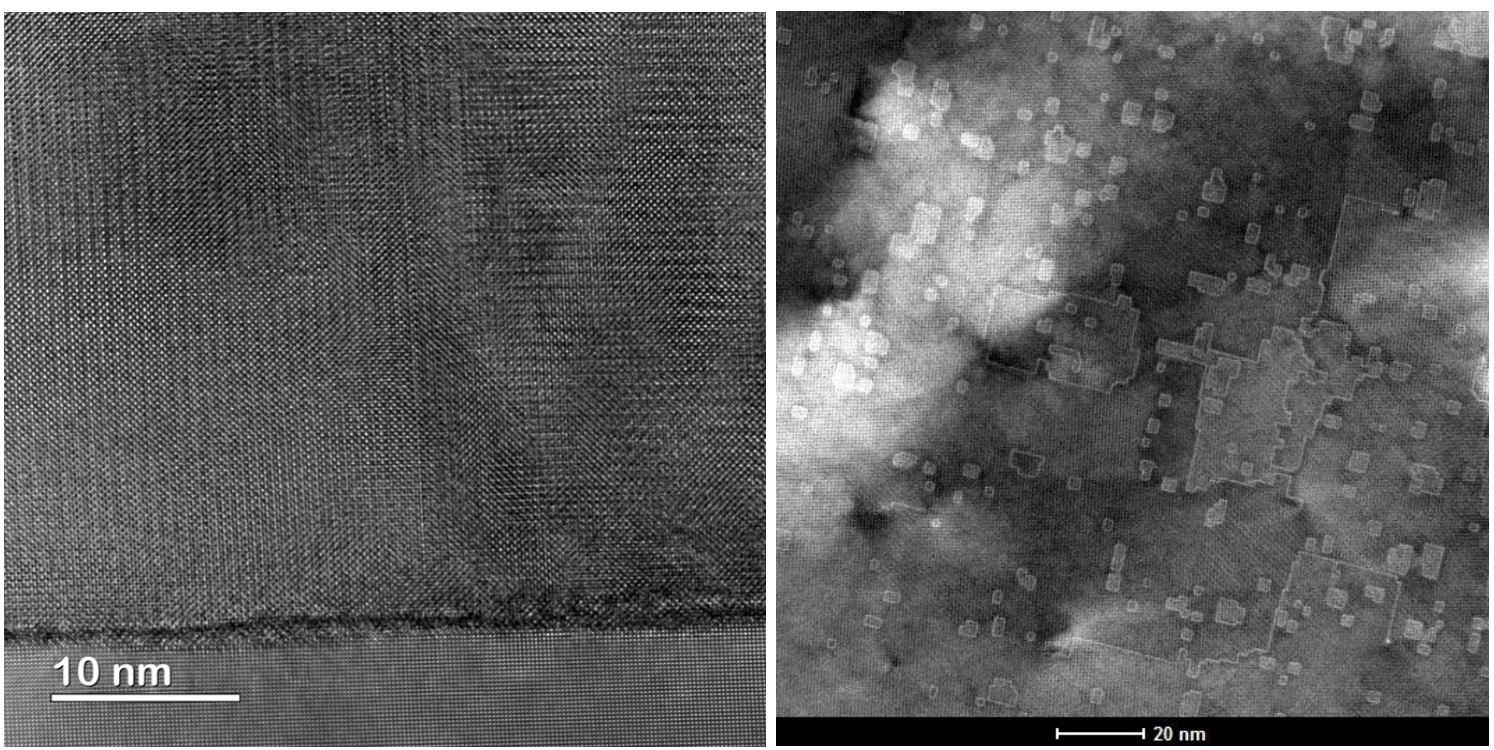

Abbildung 29 - Links ein hochaufgelöstes TEM-Bild der $x=0,75$ Probe auf $\mathrm{MgO}$. Die einkristalline Struktur kann eindeutig sichtbar gemacht werden, rechts ein HRTEM-Bild der parallel geschnittenen Lamelle 


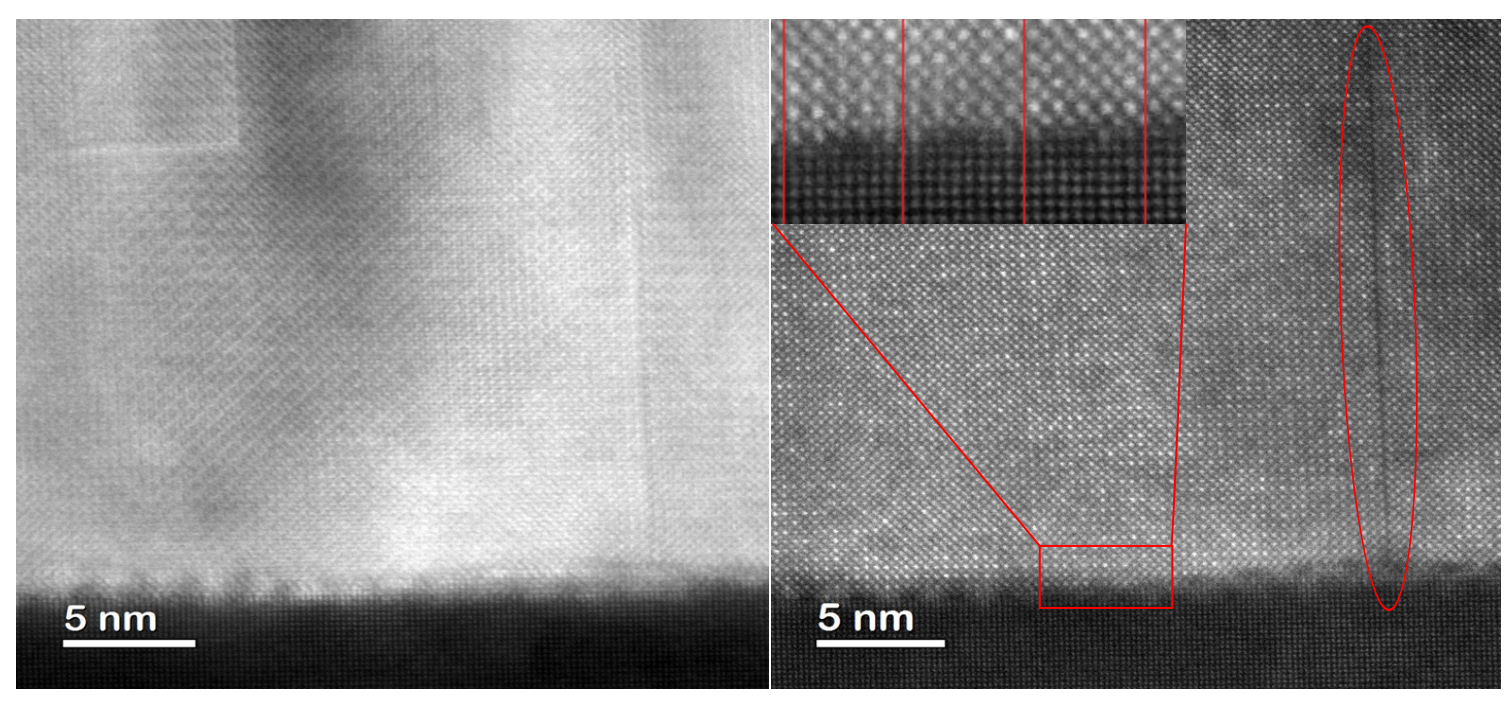

Abbildung 30 - STEM Bild mit Ruddelson-Popper-Fehler Antiphasengrenze (rote Ellipse). Links BF, rechts DF. Im Inset ist außerdem die domain-matching-epitaxy zu sehen. 
Es wurden zwei unterschiedliche Lamellen von Vladimir Roddatis präpariert. Eine Lamelle, deren Ausrichtung senkrecht zum Substrat liegt, deren Beugungsbild in Abb. 31 oben zu sehen ist. Sowie eine weitere Lamelle, deren Ausrichtung parallel zum Substrat liegt, deren Beugungsbild in Abb. 31 unten zu sehen ist. Beide Lamellen wurden im TEM abgekühlt und bei tiefen Temperaturen vermessen. Hierbei zeigt sich, dass selbst bei Raumtemperatur in der senkrechten Lamelle zusätzliche Reflexe vorliegen, sowie eine Unordnung entlang der Kristallachsen. Dies ist evident durch die langgezogenen Streifen entlang der Achsen. Die Intensität dieser Streifen wird bei tieferen Temperaturen noch stärker. Die Gitterkonstante ändert sich hierbei von $a=5,345$ bei $\mathrm{T}=300 \mathrm{~K}$ zu $a=5,331$ bei $\mathrm{T}=136 \mathrm{~K}$. Auf dem Beugungsbild ist außerdem der (200) Peak des MgO zu sehen, in Abb. 31 durch das blaue Quadrat veranschaulicht. Diese Peaks sind natürlich für die Lamelle parallel zum Substrat nicht zu sehen. Anhand des MgO (200) Peaks ist außerdem zu erkennen, dass neben den zu erwartenden zwei Peaks $(020)_{\text {Pnma }}$, zwischen MgO-Peak und Zentralpeak und (040) Pnma direkt neben dem MgOPeak, weitere Peaks auftauchen bei $(010)_{P n m a}$ un $(030)_{P n m a}$, die nach dem Strukturfaktor ausgelöscht sein sollten.

Schaut man sich die parallel geschnittene Lamelle an, fällt sofort auf, dass es schwache senkrechte Striche gibt bei Raumtemperatur. Der senkrechte Strich in der Mitte des Beugungsbildes stammt von der Übersättigung des Detektors. Gehen wir hingegen zu tieferen Temperaturen ändert sich dieses Bild. Die parallele Lamelle zeigt nun ebenfalls Streifen. Die Orientierung scheint jedoch in einem $45^{\circ}$-Winkel zu den Achsen zu liegen. Das Bild einer kommensurablen Ladungsordnung, des vorher vorgestellten Wigner-Crystal Modells mit einer Vervierfachung der a-Achse kann nicht bestätigt werden. Auch in der Veröffentlichung von Mori Chen und Cheong [45] zeigt sich bei der Dotierung $\mathrm{x}=0,75$ keine Wigner Struktur. Das in ihrer Arbeit beobachtete Bi-Stripe Modell konnte in den hier vorliegenden Messungen aber ebenfalls nicht beobachtet werden. Die Gitterkonstante von LCMO in der parallelen Lamelle ändert sich hierbei von $a=5,345 \mathrm{zu} a=5,252$ in beide Richtungen. Es muss beachtet werden, dass im TEM Gitterkonstanten nicht gemessen werden können. Für die Bestimmung der Gitterkonstanten wurden die Abstände der Gitterpunkte verwendet, die bei gleichem Abstand der Kamera einen Vergleich ermöglichen sollten. Es ist ebenfalls eine Bestimmung möglich, wenn man die Gitterkonstante des $\mathrm{MgO}$ als gegeben ansetzt. Die hieraus resultierende Gitterkonstante ist aber aufgrund der geringen Anzahl der Reflexe und der Auflösung des Bildes ungenauer als die erste Variante. Die über die MgO Gitterkonstante bestimmte Größe von $a=5,34+/-0,03$ entspricht dennoch der vorher aus XRD bestimmten Gitterkonstante. 

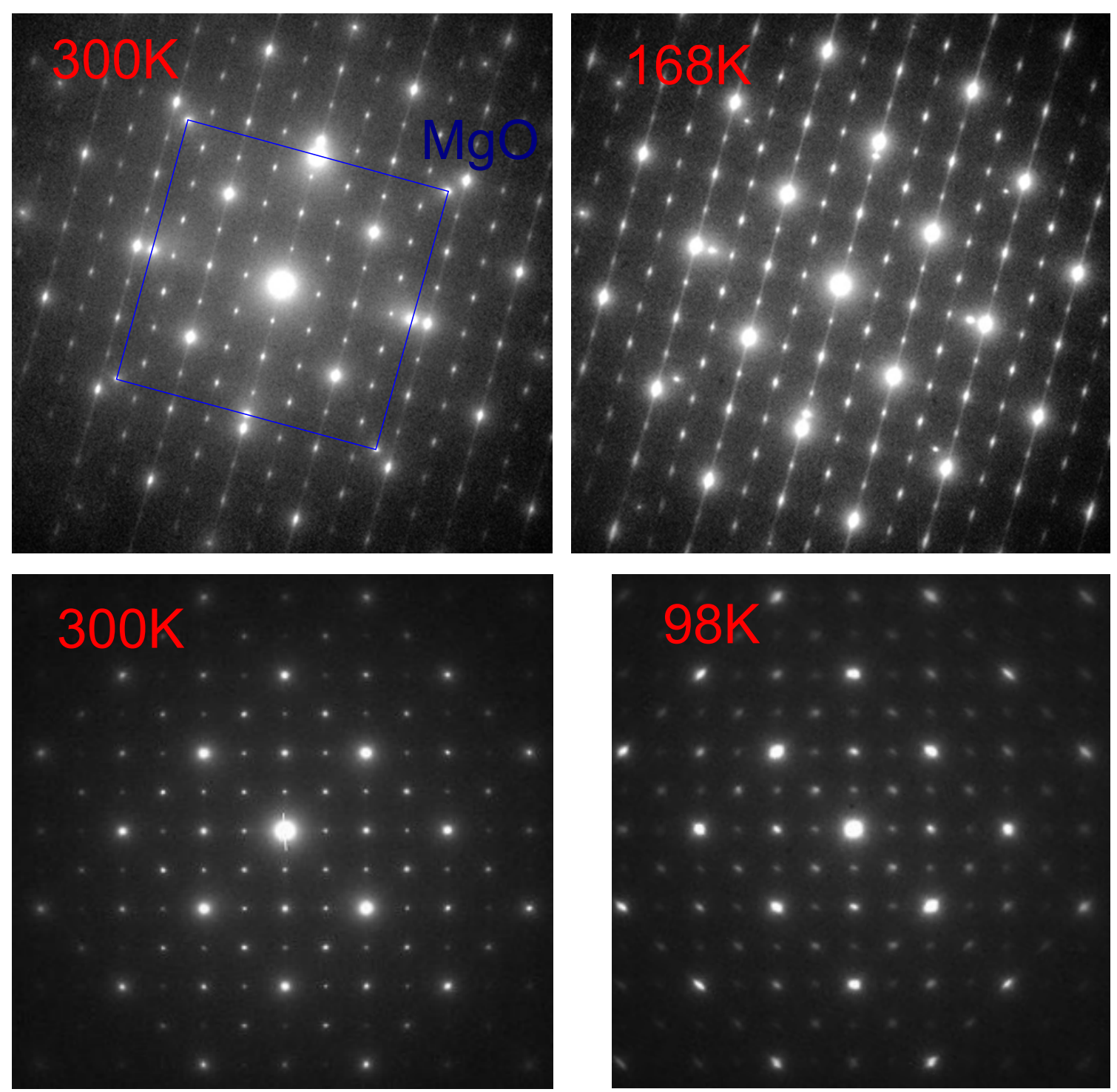

Abbildung 31 - Oben die Beugungsbilder der TEM-Lamelle, die senkrecht zum Substrat geschnitten wurde. Unten die Beugungsbilder der Lamelle parallel zum Substrat 


\subsection{Oberflächenmorphologie (STM)}
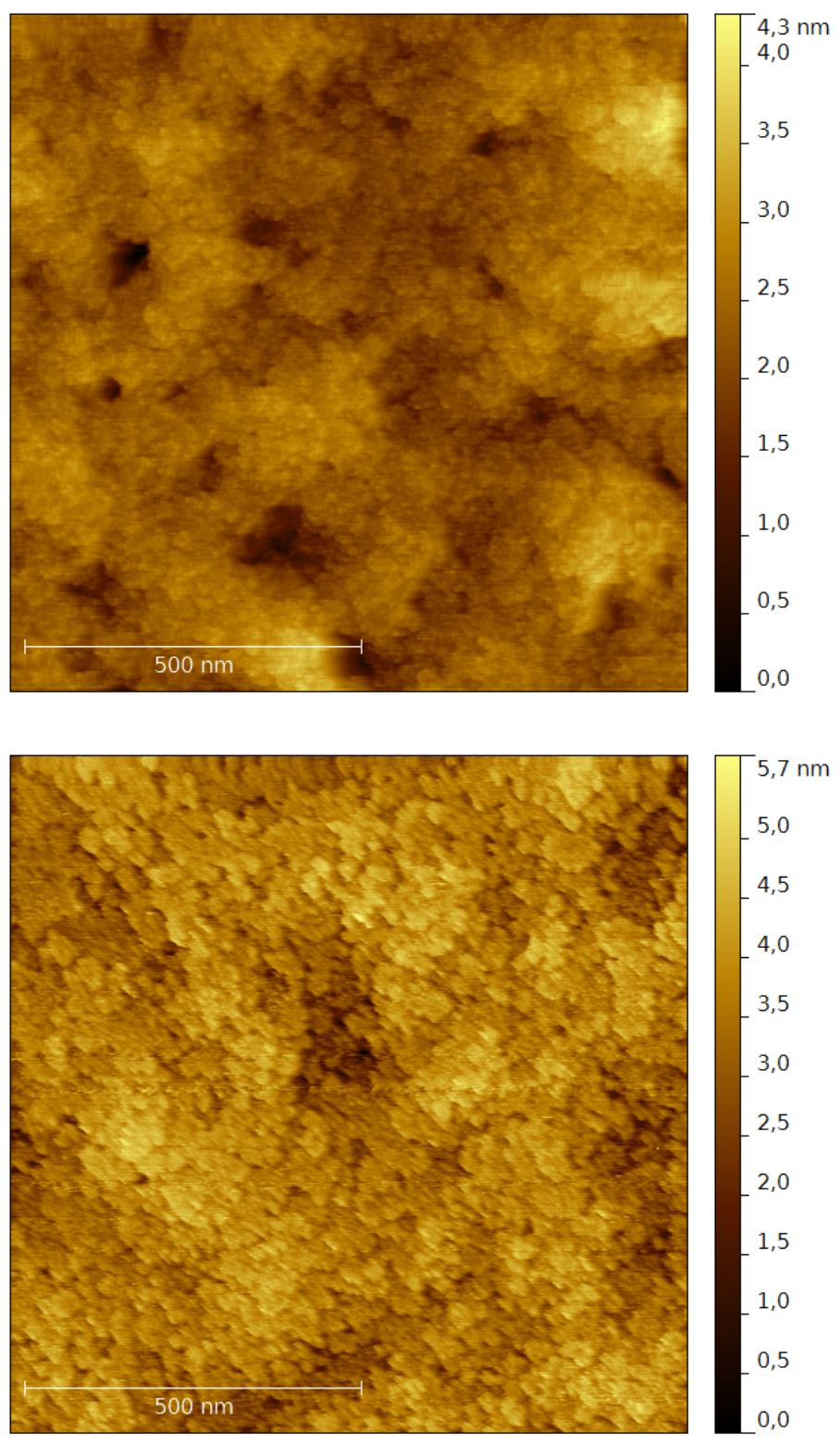

Abbildung 32 - STM Aufnahmen zweier LCMO Schichten auf MgO mit $x=0,75$ auf $1 * 1 \mu m^{2}$ und mit $x=0,66$ auf $1 * 1 \mu m^{2}$ 
Die STM Aufnahmen, der auf MgO gewachsenen Schicht, weisen auf ein Frank-van-der-Merwe-Wachstum hin. Die Filme wachsen dabei Lage per Lage. Dadurch ergibt sich eine extrem glatte Oberfläche, deren Eigenschaften nahezu unabhängig von der Schichtdicke sind. Durch das verspannte Wachstum der Schicht am Substrat bilden sich zur Entspannung Versetzungen |74|, diese können in den TEM-Messungen gezeigt werden. Durch diese Versetzungen raut die Oberfläche der Schicht zuerst auf, um dann ein unverspanntes Wachstum zu zeigen. Die Schichten sind damit von der Oberflächenbeschaffenheit mit den LSMO Schichten der MAD zu vergleichen. Für die höherdotierten LCMO Schichten ist es schwierig ein STM Bild aufzunehmen, da die geringe Leitfähigkeit der Schichten oft Artefakte produziert. Die Schichten zeigen bei einer Dicke von $d=425 \mathrm{~nm}$ eine RMS Rauigkeit von 0,4-0,5 nm.

\subsection{Bestimmung der Schichtdicke durch SEM}

Nachdem alle anderen Messungen an den Proben abgeschlossen waren, wurden die Proben in der Mitte gespalten, um die Schichtdicke zu bestimmen. Dies ist nötig, da die Schichten für Kleinwinkelmessungen im Röntgen viel zu dick sind. Die Aufnahmen wurden mit dem Detektor für Sekundärelektronen aufgenommen. Man kann anhand des Kontrastes zwischen Film und Substrat nun die Dicke des Films bestimmen. Aufgrund der SEM Aufnahmen für mehrere Proben ergibt sich eine Dicke von $d=518+/-20 \mathrm{~nm}$ für Proben die in der MAD1 hergestellt wurden. Trotz der geringen Beschleunigungsspannung von nur 5kV konnten in der Nähe des Substrats Aufladungseffekte beobachtet werden, die die Genauigkeit der Schichtdickenmessung verfälschen. In Abbildung 33 ist ein Beispiel einer solchen Schichtdickenmessungen zu sehen.

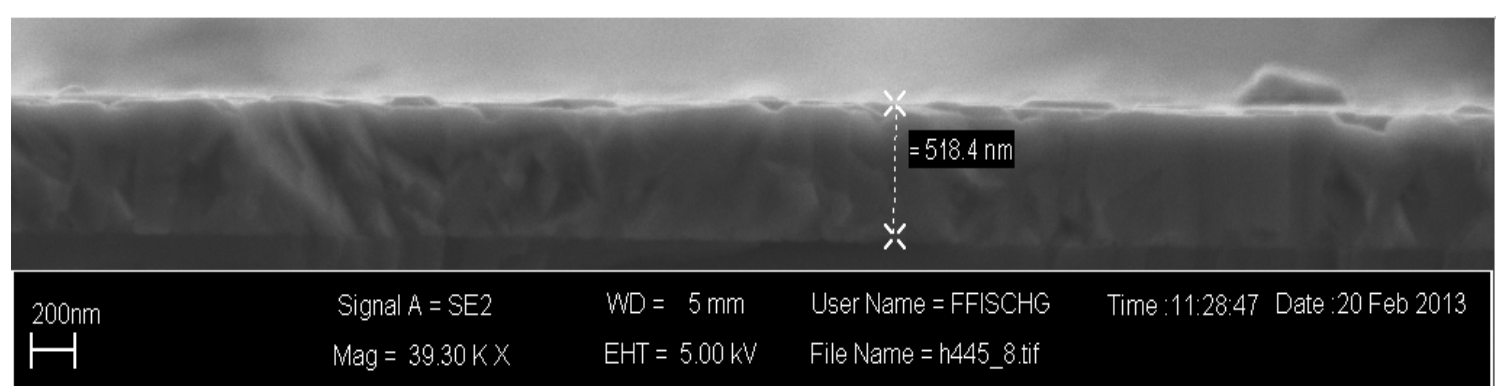

Abbildung 33 - Seitliche Aufnahme einer LCMO Schicht $x=0,75$ auf $M g O$, die in der MAD1 hergestellt wurde, mit dem SEM. Über den Kontrast der Helligkeiten läst sich die Schichtdicke bestimmen 


\subsection{Transportmessungen mit dem PPMS}

Die Widerstandsmessungen an den LCMO Schichten erfolgten im PPMS als Vierpunktmessungen. Hierbei werden in gleichem Abstand in einer Linie vier Kontakte auf einer Probe angebracht. Für eine Messung bei Gleichspannung ignoriert diese Geometrie den Widerstand der Kontakte, da sich die Potentiale der Kontakte aufheben [59|. Als Messparameter für die Widerstandsmessung wurden $U_{\max }=95 \mathrm{mV}$ und $I_{\max }=50 \mu \mathrm{A}$ verwendet. Typischerweise wurde von $T=400 \mathrm{~K}$ heruntergekühlt bis $\mathrm{T}=10 \mathrm{~K}$. Hierbei wurde ständig der Widerstand gemessen. Der Widerstandsverlauf abhängig von der Temperatur ist in Abbildung 34 dargestellt. Für Dotierungen zwischen $\mathrm{x}=0,55-0,85 \mathrm{kann}$ ein allgemeines Verhalten beobachtet werden. Die Proben haben bei 400K einen geringen spezifischen Widerstand, von $\rho \approx 10^{-4}$, der gut zum Bild des entarteten Halbleiters passt [75]. Der Widerstand ist über einen großen Temperaturbereich nahezu konstant, auch dies in guter Übereinstimmung mit der Annahme eines entarteten Halbleiters. Unterhalb einer dotierungsspezifischen Temperatur zeigen die Proben eine starke Änderung der Steigung. Die Proben ändern ihr Verhalten innerhalb weniger Kelvin von einem schlechten Leiter $\mathrm{zu}$ einem guten Isolator, möglicherweise aufgrund der einsetzenden starken Lokalisierung der Elektronen in der Überstruktur der Ladungsordnung. Dies ist auch der Grund warum die Widerstandsmessungen ab einer gewissen Temperatur abbrechen, da die Stromquelle keine größeren Messspannungen anlegen kann und somit die Sättigung bei ungefähr $2 M \Omega$ erreicht wird. Der spezifische Widerstand der Proben lässt sich aus der Messgeometrie ableiten. Hierbei wird von einer Schichtdicke $d=500 \mathrm{~nm}$ ausgegangen. Der Abstand der Kontakte sei mit $\mathrm{L}=2 \mathrm{~mm}$ gegeben. Die Breite, in der der Strom fließt, wird mit $b=2 \cdot L$ angenommen. Dadurch ergeben sich dann die hier errechneten Werte aus den gemessenen Widerständen. In Abbildung 34 ist als Inset $\frac{d l n(\rho)}{d T^{-1}}$ geplottet. Der Verlauf polykristalliner Proben $\mid 76$ deckt sich gut mit dem der Filme auf MgO. Allerdings weisen die Filme einen geringeren Widerstand bei $\mathrm{T}=400 \mathrm{~K}$ auf und zeigen keinen deutlichen Ladungsordnungsübergang, wie er bei den Polykristallen als Plateau im Widerstand sichtbar wird. Dies heißt aber nicht, dass die Filme keine Ladungsordnung zeigen, da sie bei Auftragung von $\frac{d \ln (\rho)}{d T^{-1}}$ gegen $\mathrm{T}$ deutlich als Peak bei $T_{C O}$ sichtbar wird. Sobald die Dotierung den Bereich der Ladungsordnung überschreitet zeigen die Filme erst bei Temperaturen unterhalb von $\mathrm{T}=100 \mathrm{~K}$ einen stark isolierenden Charakter. Die Erhöhung des Widerstands in diesem Temperaturbereich kann möglicherweise durch thermisches Blocken der C-AFM Bereiche erklärt werden, die über den Superaustausch effektiv die Leitfähigkeit verringern [39]. Außerdem zu bemerken ist, dass die elektronischen Eigenschaften der Proben mit Dotierungen nahe $\mathrm{x}=1$ extrem stark variieren und 
eine kleine Dotierungsänderung daher eine große Änderung des Widerstands bewirkt [77.

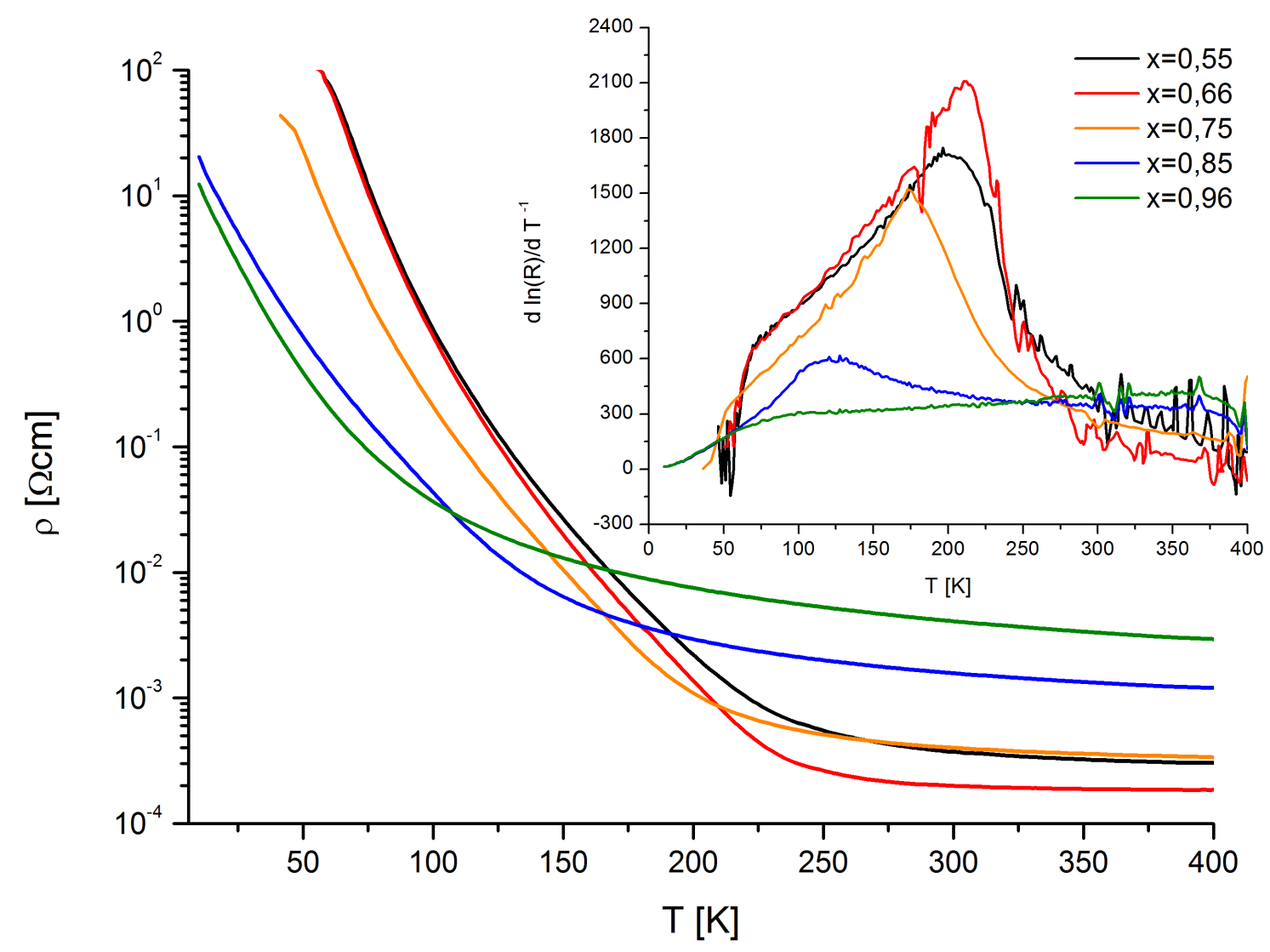

Abbildung 34 - Widerstandsmessung der Filme mit $x=0,55-0,96$ mit dem PPMS von $T=10-400 K$. Unterhalb von $T=50 \mathrm{~K}$ kann für viele Dotierungen kein Widerstand mehr gemessen werden, da die Widerstände zu groß für die DC-Option des PPMS sind. Als Inset ist der Koeffizient $\frac{d l n(\rho)}{d T^{-1}}$ abhängig von der Temperatur für die fünf Dotierungen geplottet. 


\subsection{Magnetische Charakteristika}

Als weitere Möglichkeit die Ladungsordnungstemperatur zu bestimmen kann bei reinen Proben die Magnetisierung verwendet werden. Die Magnetisierung zeigt abhängig von der Ladungsordnungstemperatur des Systems einen Peak bei der Temperatur $T_{C O}$ [76,78, da die Ladungsordnung auch eine orbitale Ordnung bewirkt. Alle $\mathrm{M}(\mathrm{T})$ Messungen wurden dabei bei $\mathrm{H}=1000$ Oe durchgeführt. Die Proben wurden hierbei im Feld eingekühlt (FC) und die Magnetisierung vermessen. Das Problem von magnetischen Messungen an dünnen Schichten ist im Allgemeinen das große Moment des Substrats, das gerade für LCMO Proben, die ein sehr kleines Moment zeigen, im Bereich der Ladungsordnung die eigentliche Magnetisierung der Schicht überdeckt. Um dennoch eine Auswertung der Magnetisierungsdaten durchzuführen ist das MgO Substrat vor der Deposition im SQUID vermessen worden. Die Magnetisierung des Substrats und der Kapsel kann dann von der Magnetisierung, die nach der Deposition mit Schicht gemessen wird, abgezogen werden. Dieses Verfahren erlaubt es das Moment der Schicht von dem des Substrats zu trennen. Leider kann nicht davon ausgegangen werden, dass das Substrat nach der Deposition bei der es auf $\mathrm{T}=1000^{\circ} \mathrm{C}$ aufgeheizt wird weiterhin dieselben magnetischen Eigenschaften hat. Daher sollten die Absolutwerte nicht als Auswertungsgrundlage verwendet werden. Die Auswertung erfolgt durch Subtraktion des paramagnetischen Anteils des Substrats. Das diamagnetische Moment der Kapsel und des Substrats bei hohen Temperaturen wird arbiträr gewählt um eine Skalierung auf null zu erreichen. In Abbildung 35ist die Magnetisierung für die Proben mit $\mathrm{x}=0,66$ und $\mathrm{x}=0,75 \mathrm{zu}$ sehen. Beide Graphen wurden nach dem oben beschriebenen Verfahren erstellt. Es ist offensichtlich, dass beide Proben einen Peak nahe der aus dem Phasendiagramm von Pissas entnommenen Ladungsordnungstemperatur zeigen [38, 78, 79|. In Abbildung 36 ist außerdem die Magnetisierung von $\mathrm{x}=0,55 \mathrm{x}=0,85$ und $\mathrm{x}=0,96$ zu sehen. Die Probe mit Dotierung $\mathrm{x}=0,55$ zeigt das große Problem, das bei der Herstellung von Schichten nahe Halbdotierung besteht. Die Probe ist phasensepariert in einen ferromagnetischen Bereich und einen ladungsgeordneten Bereich, wie aus dem Phasendiagramm bereits zu erwarten ist. Dadurch ist nur das ferromagnetische Verhalten der Magnetisierung sichtbar, der ladungsgeordnete Beitrag ist typischerweise um drei Größenordnungen kleiner und kann daher nicht aufgelöst werden. Die Änderung der Steigung der M(T) Kurve von $\mathrm{x}=0,55$ macht es allerdings möglich auch hier die Ladungsordnungstemperatur zu bestimmen, die ebenfalls in den Widerstandsdaten sichtbar wird.

Die Probe mit $\mathrm{x}=0,85$ zeigt ebenfalls einen Peak in der Magnetisierung sowie bei tieferen Temperaturen eine starke Erhöhung des Moments. Dies ist in 
guter Übereinstimmung mit dem verkippten antiferromagnetischen Zustand, der ein kleines Nettomoment bewirkt. Dieses Nettomoment von typischerweise $0,05 \mu_{B} /$ Einheitszelle ist hier unterhalb von $T_{C-A F M}$ zu sehen [80]. Die Probe mit $\mathrm{x}=0,96$ zeigt ein größeres Moment als $\mathrm{x}=0,85$ in guter Übereinstimmung mit der stärkeren Verkippung, wodurch das Nettomoment der gekanteten antiferromagnetischen Phase vergrößert wird. Es ist außerdem kein zweiter Peak mehr vorhanden.

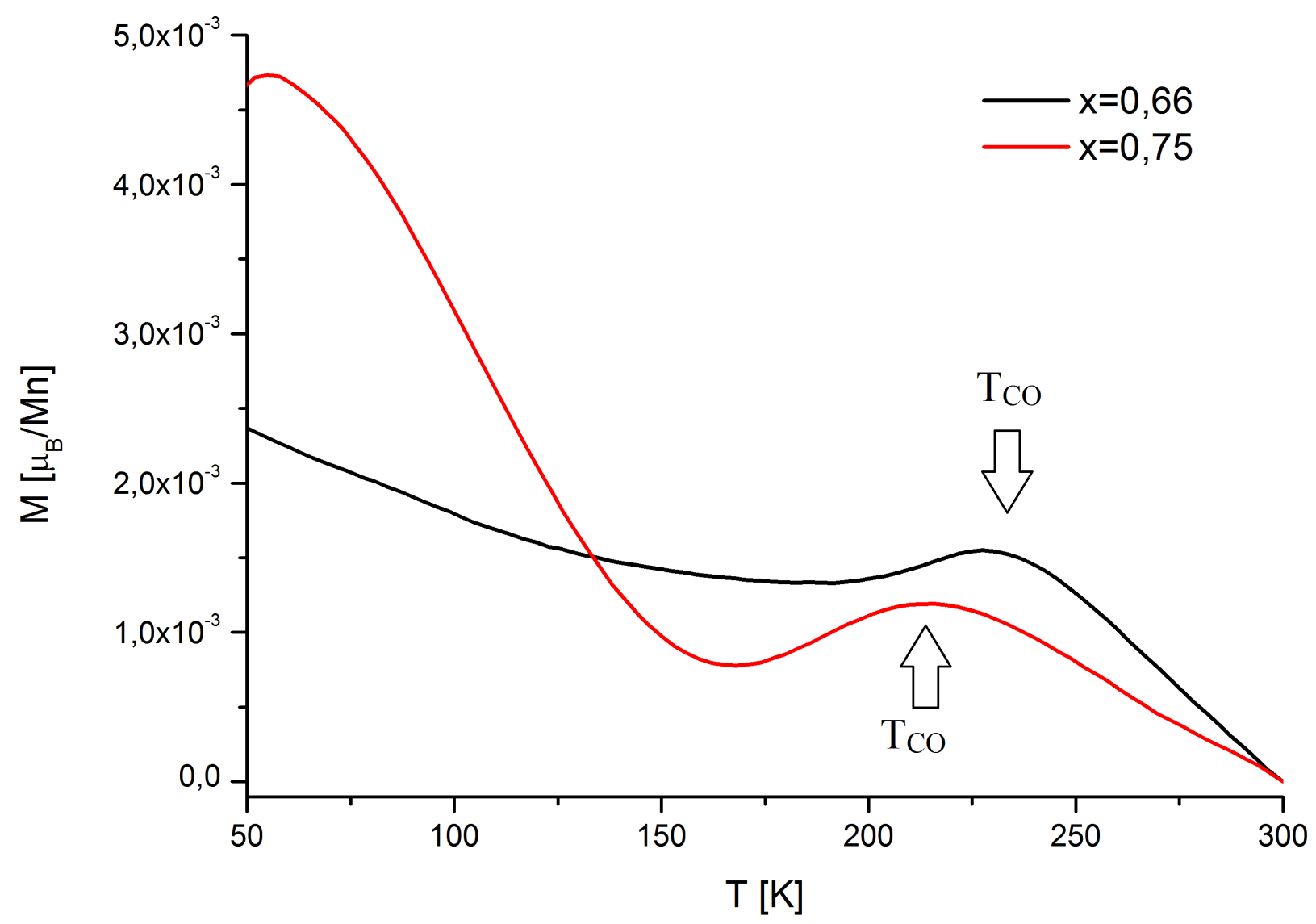

Abbildung 35 - Magnetisierung der LCMO Proben mit $x=0,66$ und $x=0,75$ auf $\mathrm{MgO}$ nach Subtraktion des Signals von $\mathrm{MgO}$ 


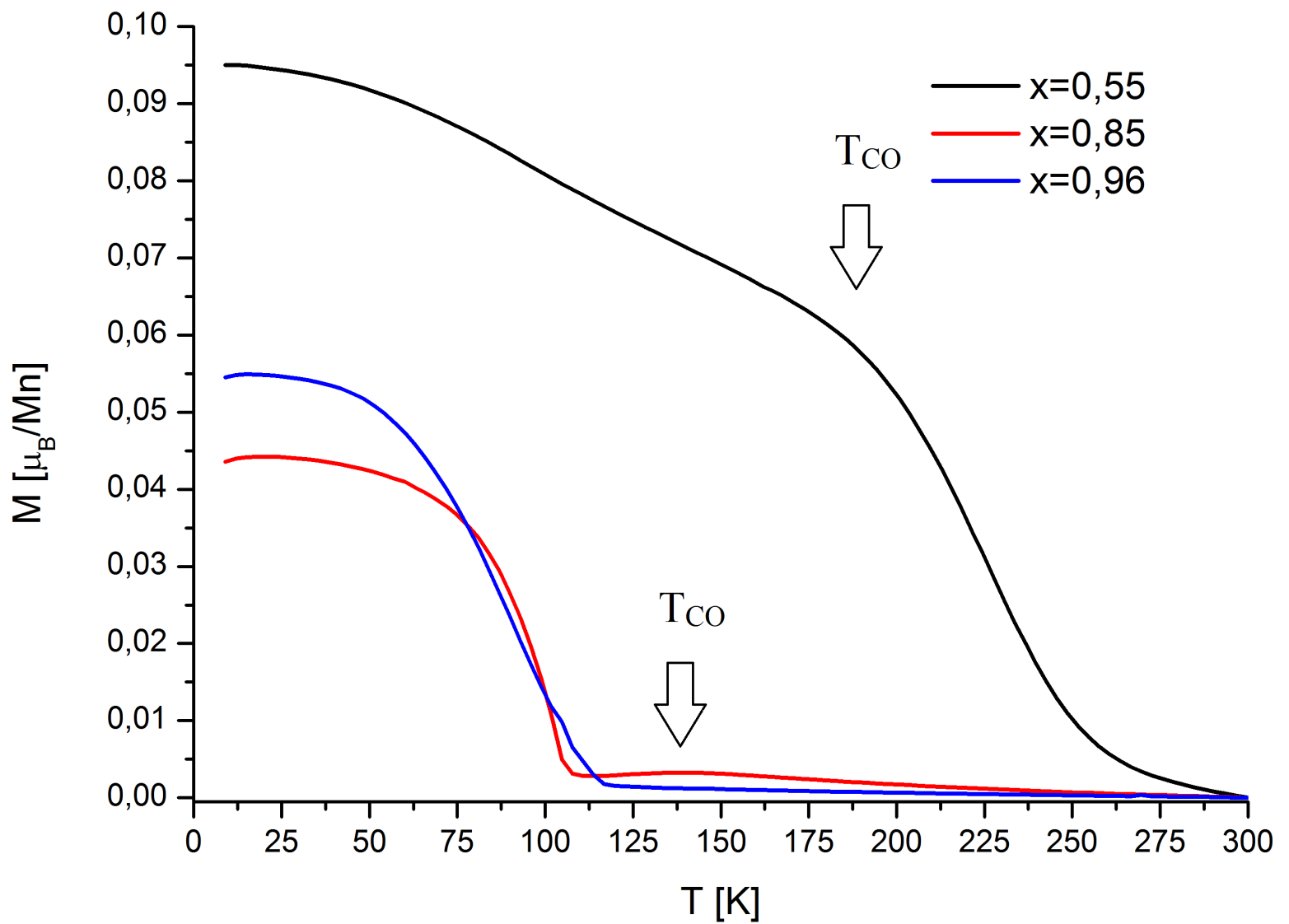

Abbildung 36 - Magnetisierung der LCMO Proben mit $x=0,55 x=0,85$ und $x=0,96$ auf $\mathrm{MgO}$ nach Subtraktion des Signals von $\mathrm{MgO}$ 
Es wurden außerdem $\mathrm{M}(\mathrm{H})$ Messungen durchgeführt für $\mathrm{x}=0,75, \mathrm{x}=0,85$ und $\mathrm{x}=0,96$, siehe Abb. 37, Abb. 38 und Abb. 39. Alle Proben wurden bei $\mathrm{T}=30-40 \mathrm{~K}$ aufgenommen und im Nullfeld eingekühlt. Der Grund für die recht hohe Temperatur ist, dass $\mathrm{MgO}$ ein superparamagnetisches Verhalten der Hysteresekurve bei sehr tiefen Temperaturen zeigt und gleichzeitig die Nullstelle der Magnetisierung soweit wie möglich vermieden werden soll, da das SQUID die Magnetisierung $\mathrm{M}=0$ oft falsch darstellt. Alle Abbildungen sind nach Abzug des diamagnetischen Untergrunds entstanden. Die Hysteresekurve der $\mathrm{x}=0,75$ Probe ist hier exemplarisch für die ladungsgeordneten Schichten dargestellt. Die Hysteresekurve der $\mathrm{x}=0,66$ dotierten Schicht wurde ebenfalls aufgenommen zeigt aber dasselbe Verhalten. Die $\mathrm{x}=0,75$ Probe zeigt ein kleines ferromagnetisches Moment und eine Hysterese. Das Koerzitivfeld kann zu $H_{C}=400 O e$ bestimmt werden. Es ist nur ein sehr kleiner Teil der Probe als ferromagnetisch anzusehen. Möglicherweise die Randbereiche der Schicht die im Röntgen nicht untersucht wurden.

Die Hysteresekurven der beiden C-AFM Proben zeigen beide eine ferromagnetische Remanenz. Die Remanenz der $\mathrm{x}=0,85$ Probe ist hierbei kleiner als die von $\mathrm{x}=0,96$. Die kleine Remanenz der Proben deutet ebenfalls auf ein Phasenseparationsszenario hin. Hierbei ist die Probe separiert in kleine ferromagnetische Bereiche, gepaart mit deutlich größeren antiferromagnetischen Bereichen. Ein rein verkanteter Antiferromagnet würde keine Remanenz zeigen, es sei denn die Drehung, der Magnetisierung in die diametral gegenüberliegende Richtung, wäre mit einer Energiebarriere verbunden. Die relativ kleinen Werte der Remanenz bedeuten, dass in beiden Proben die Anzahl der Mangan- atome, die ein ferromagnetisches Moment zeigen sehr klein ist. Es kann eine deutliche Steigerung des Koerzitivfelds mit der Dotierung festgestellt werden von $H_{C}=1400 O$ e bei $\mathrm{x}=0,85 \mathrm{zu} H_{C}=3600 O e$ bei $\mathrm{x}=0,96$. Die Probe mit $\mathrm{x}=0,96$ zeigt außerdem zwei ferromagnetische Phasen. Dies wird besonders in der Neukurve deutlich. Es ist unklar was der Grund für dieses Verhalten ist. 


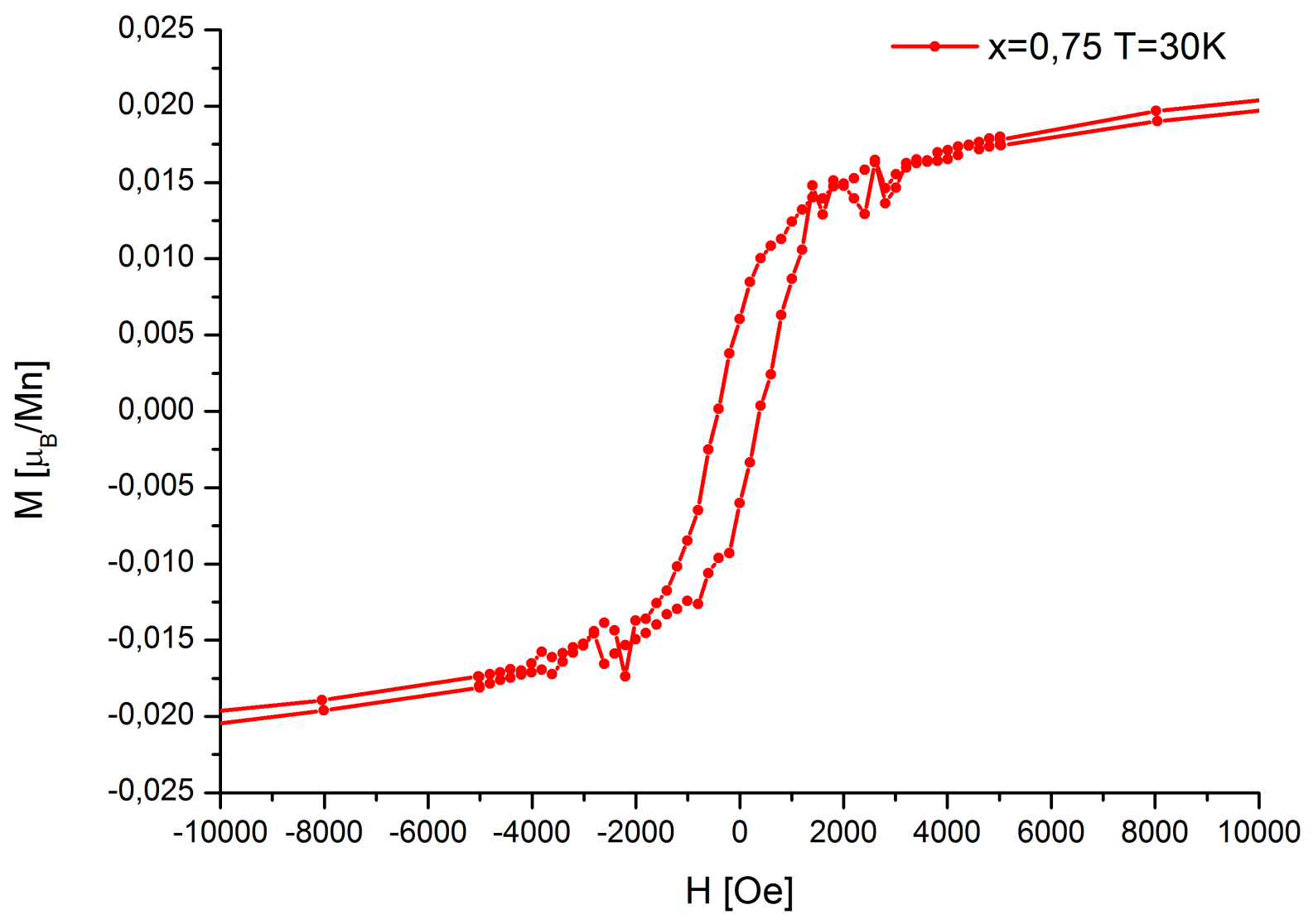

Abbildung $37-M(H)$ der $L C M O$ Probe mit $x=0,75$ auf $M g O$ nach Subtraktion des Signals von $\mathrm{MgO}$. 


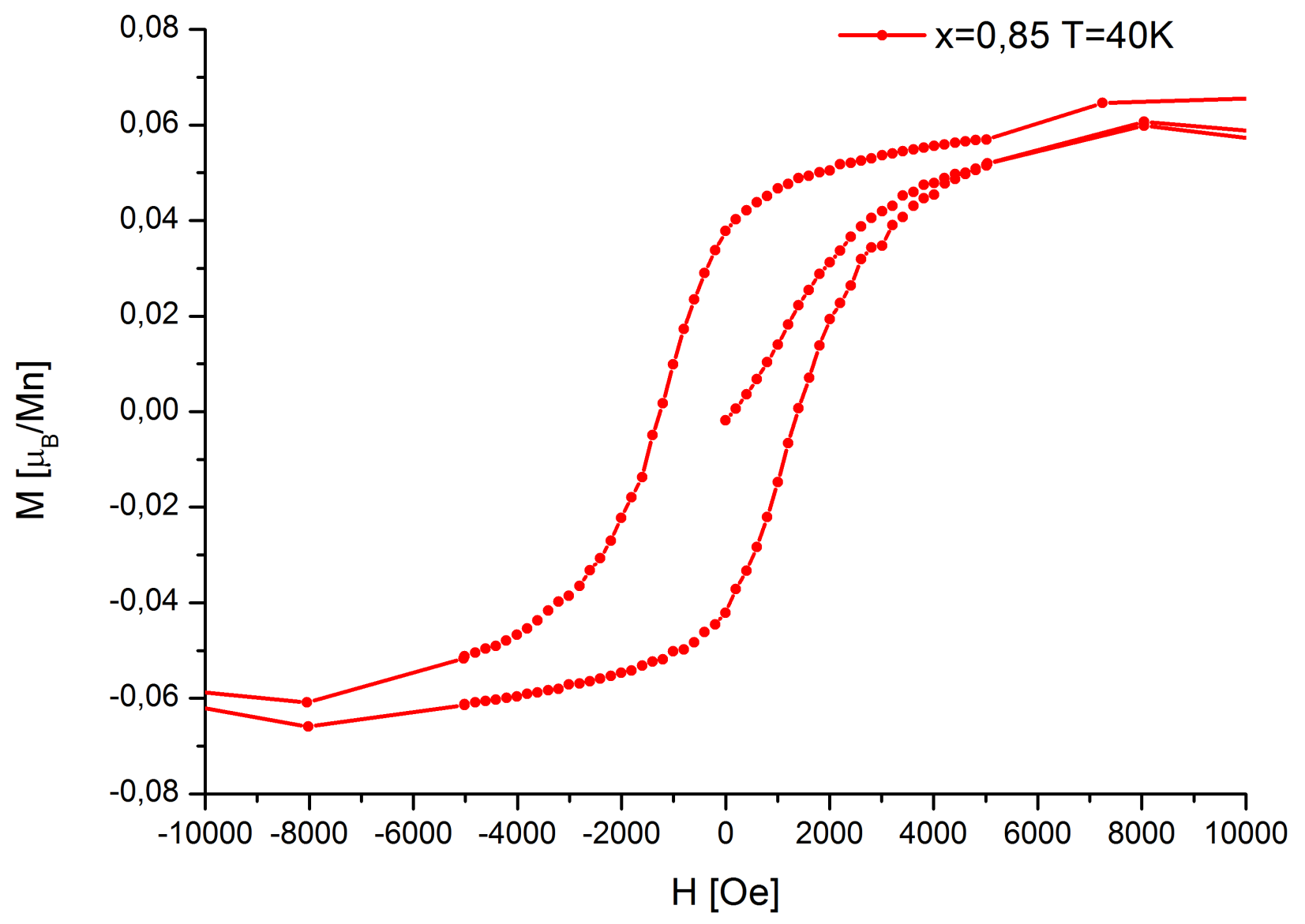

Abbildung $38-M(H)$ der $L C M O$ Probe mit $x=0,85$ auf $M g O$ nach Subtraktion des Signals von $\mathrm{MgO}$. 


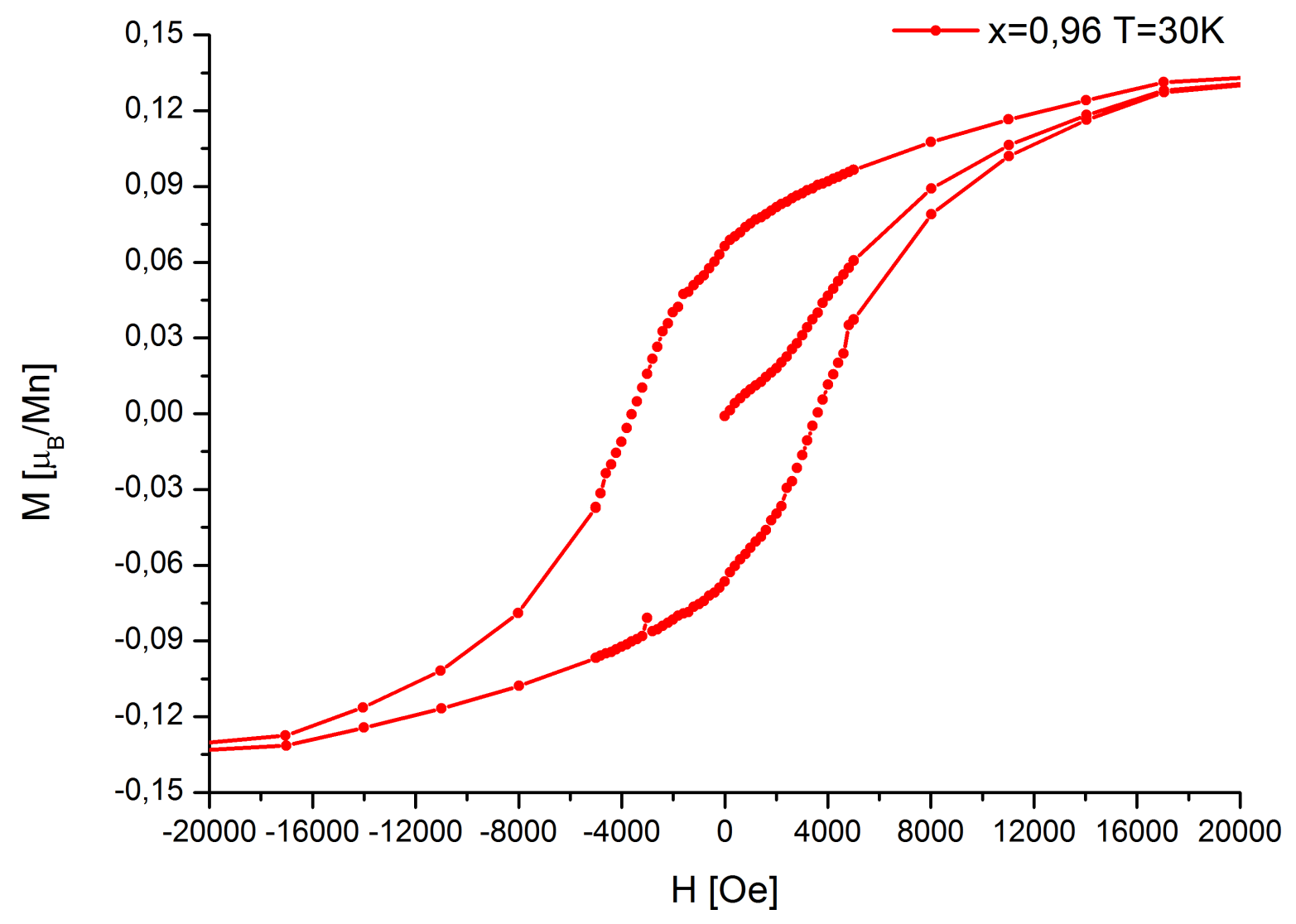

Abbildung $39-M(H)$ der $L C M O$ Probe mit $x=0,96$ auf $M g O$ nach Subtraktion des Signals von $\mathrm{MgO}$. 


\subsection{Optische Eigenschaften}

\subsubsection{THz-Spektroskopie}

Die THz-Messungen, die in dieser Arbeit gezeigt werden, wurden alle von Elena Zhukova und Boris Gorshunov in Stuttgart aufgenommen und ausgewertet im Rahmen des DFG Projekts DR 228/36. Dabei wurden aus Göttingen Proben bereitgestellt, deren THz-Daten dann in Stuttgart aufgenommen und ausgewertet wurden. Hier soll nun ein kurzer Überblick über die erzielten Ergebnisse gegeben werden. Der Frequenzbereich für die THz-Messungen reicht von $\nu=30-1200 \mathrm{GHz}$. Die THz-Spektroskopie von vorher untersuchten Polykristallen zeigt, dass die hochdotierten LCMO Proben einen Bosonpeak besitzen und eine Überstruktur beobachtet werden kann, die sich als Peak in der Leitfähigkeit durch Zurückfalten auf den $\Gamma$-Punkt manifestiert. Misst man weiter bis in den FIR-Bereich, also oberhalb von $\nu=1,2 \mathrm{THz}$, so zeigen sich weitere Peaks, die durch weitere Reflektionen an der BrillouinZonengrenze auftreten.

Um nun auszuschließen, dass die gemessenen Ergebnisse von der polykristallinen Struktur der Keramiken herrühren, sollten überdotierte einkristalline Schichten hergestellt werden. Die freistehenden Filme zeigen einen kleinen Peak in der Leitfähigkeit unterhalb von $T=40 K$. In den Keramikproben, die vorher vermessen wurden, zeigt sich der Peak ab einer Temperatur von $T=100 K$. Nicht nur die Intensität des Peaks, sondern auch die Position verschiebt sich in Abhängigkeit von dem untersuchten Probensystem. Während der Peak für die Keramiken bei $20 \mathrm{~cm}^{-1}$ liegt, wandert er für die freistehenden Filme nach $25 \mathrm{~cm}^{-1}$. Die Energie der niedrigsten van-Hove-Singularität liegt bei $30 \mathrm{~cm}^{-1}$. Es zeigt sich, dass die Stärke der Wechselwirkung, aus dem Modell von Gurevich et. al., der ungeordneten Bereiche von den Keramiken, über die freistehenden Filme abnimmt.

Ein Ausfrieren der Phononen führt im Allgemeinen zum quadratischen Verhalten bei niedrigen Wellenzahlen für tiefe Temperaturen. Der Peak in der Leitfähigkeit bei tiefen Temperaturen kann durch diesen Effekt jedoch nicht erklärt werden, da insbesondere rechts des Peaks eine zu große Intensität vorliegt.

Damit wird deutlich, dass der Boson-Peak wahrscheinlich durch die Unordnung in den Polykristallen entsteht und das Materialsystem selber, wenn überhaupt nur eine geringe Unordnung aufweist, da der Peak in den kristallineren freistehenden Filmen deutlich kleiner ist.

Die Überstrukturpeaks können nur anhand der FIR-Messungen ausgewertet werden, da die Oszillationen zu tief liegen um mit THz vermessen zu werden. Leider wurden aufgrund eines Umbaus des Labors nur die ersten Schich- 
ten mit $\mathrm{THz}$ vermessen. Es kann somit nicht ausgeschlossen werden, dass in diesen ersten Schichten Defekte auftreten, die in den später hergestellten Schichten, belegt durch TEM, nicht vorhanden sind.

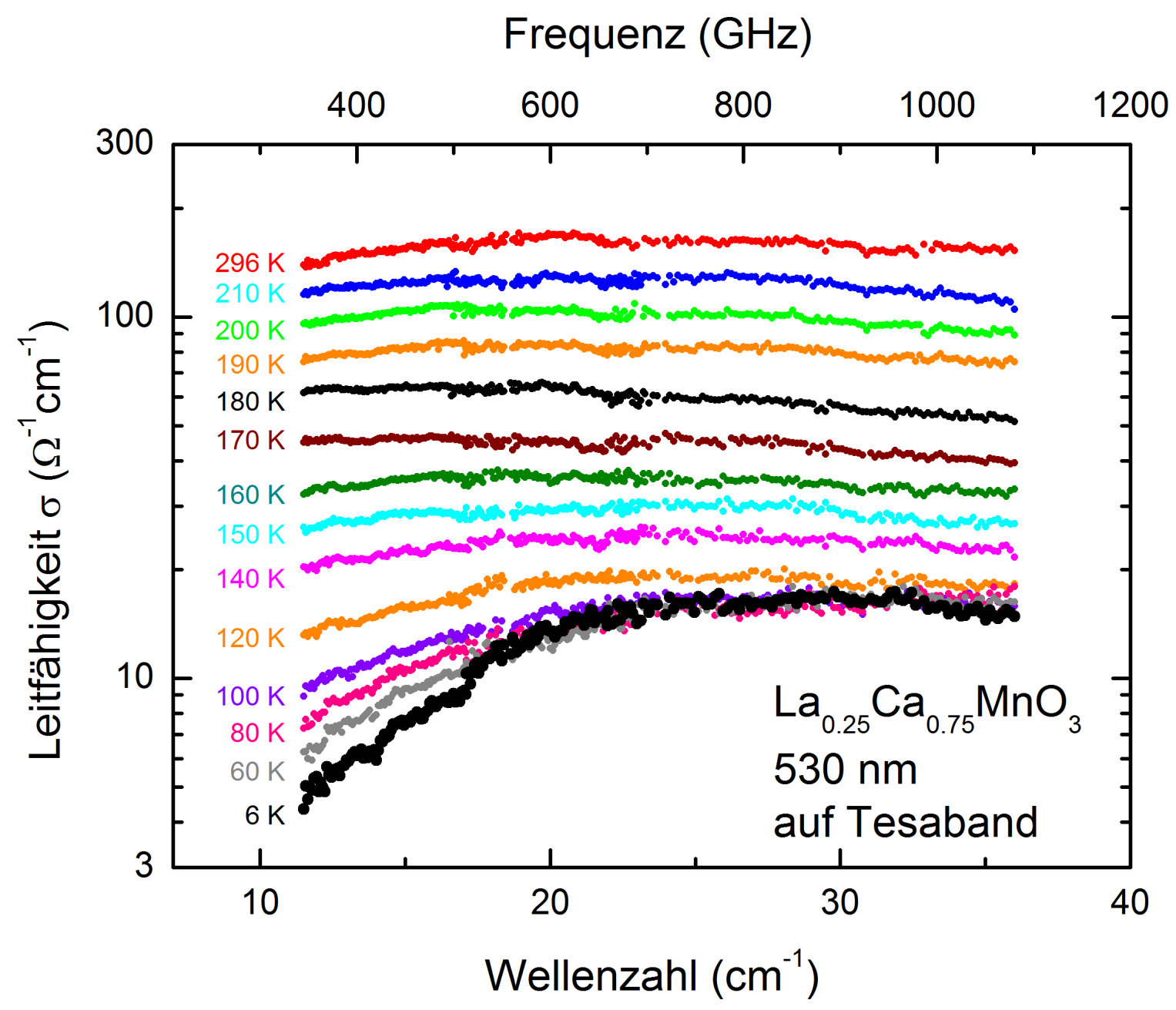

Abbildung 40 - Spektrum der optischen Leitfähigkeit eines LCMO Films mit $x=0,75$ auf Tesaband nach der Lagerung in $\mathrm{NaOH}$ 


\subsubsection{FIR-Spektroskopie}

Die auf MgO hergestellten Filme konnten nicht durch die FIR-Spektroskopie vermessen werden. Der Grund hierfür ist das MgO Substrat, wie bereits eingehend erwähnt. Die starke Infrarotabsorption der Phononmoden des Substrats macht es damit unmöglich die im Vergleich zum Substrat tausendmal dünneren Proben zu vermessen. Dies war der Grund warum freistehende Filme hergestellt werden mussten. Andere Substrate waren auch im Gespräch, aber alle in Frage kommenden Substrate zeigen starke Infrarotabsorptionslinien und würden damit das Signal zu stark verfälschen. Die hergestellten freistehenden Filme wurden ebenfalls in Kooperation mit der Universität Stuttgart von Boris Gorshunov und Elena Zhukova vermessen. Auch die Auswertung der Spektren erfolgte dabei in Stuttgart. Die Filme wurden wie im vorher beschriebenen Verfahren von den Substraten mit einem Tesastreifen gelöst. Derselbe Tesastreifen wurde nachdem er in $\mathrm{NaOH}$ ausgelagert wurde im FIR-Spektrometer vermessen. Dies ist nötig, da der Film und der Tesafilm ein Zweilagensystem bilden und die Leitfähigkeit des Tesafilms abgezogen werden muss. Das Ergebnis ist in Abb. 41zu sehen. Das Ergebnis stellt die ersten freistehenden Manganatfilme dar, die mit FIR-Spektroskopie vermessen werden konnten [81. In Abb. 42 sind die

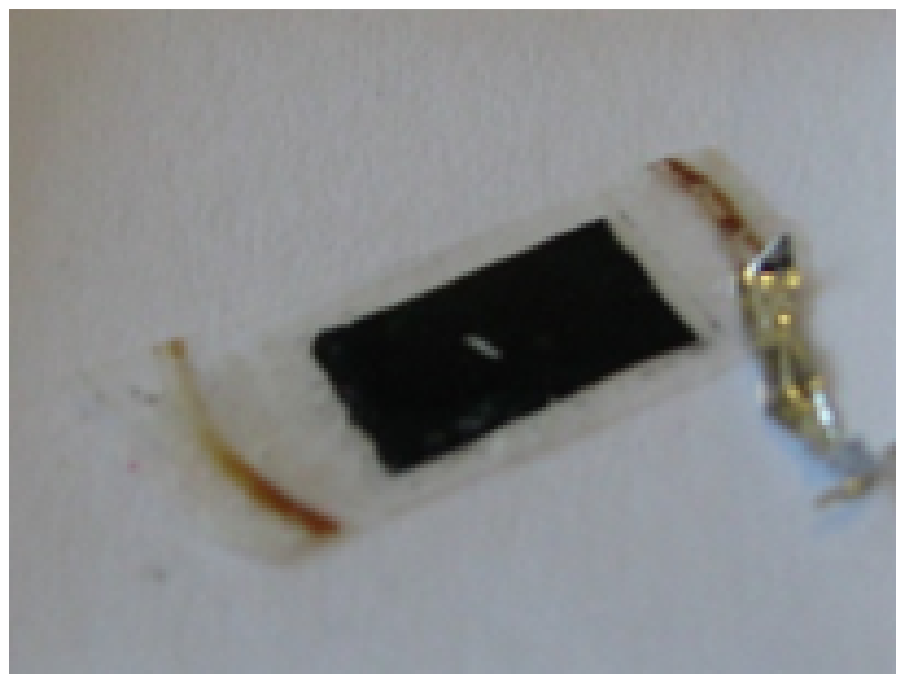

Abbildung 41 - Foto eines in Stuttgart präparierten $x=0,75$ Films

Spektren der drei Dotierungen $\mathrm{x}=0,5 \mathrm{x}=0,75$ und $\mathrm{x}=0,85$ nach Abzug des Beitrags des Tesas dargestellt. In Abb. 43 , Abb. 44 und Abb. 45 sind die Transmission für drei unterschiedliche Dotierungen der freistehenden Filme mit der Transmission der Keramiken verglichen worden. Es ist zu erkennen, dass das FIR-Spektrum der freistehenden Filme, nach Abzug des Tesafilmspektrums, dem Spektrum der vorher gemessenen Keramiken sehr ähnlich ist. Bezeichnend ist, dass die freistehenden Filme mehr und stärkere Infrarotlinien zeigen und sich die allgemeinen Werte der Transmission stark unterscheiden. 


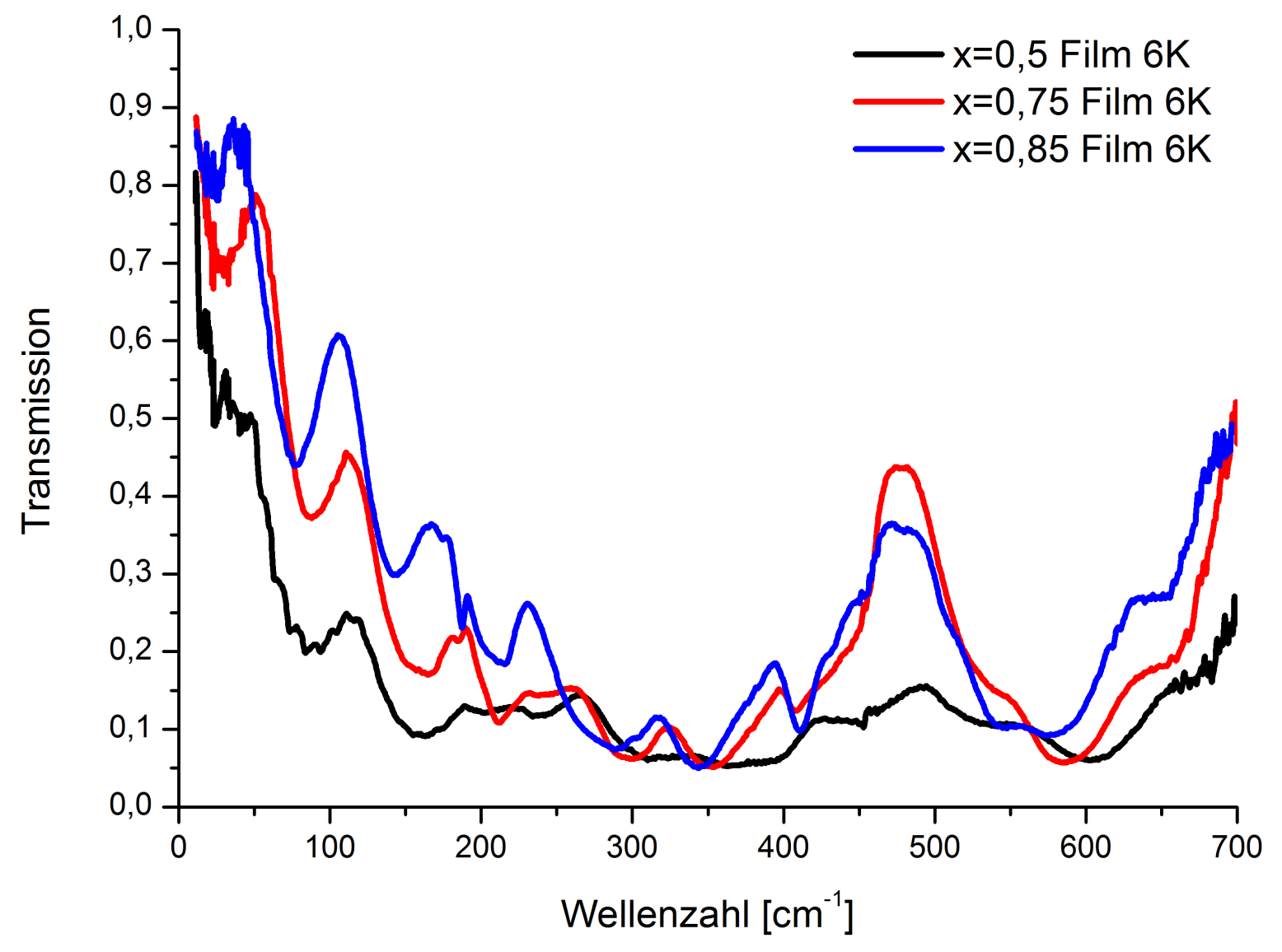

Abbildung 42 - FIR-Spektrum der LCMO Filme mit $x=0,5, x=0,75$ und $x=0,85$ nach Abzug des Tesabandspektrums bei $T=6 \mathrm{~K}$ 


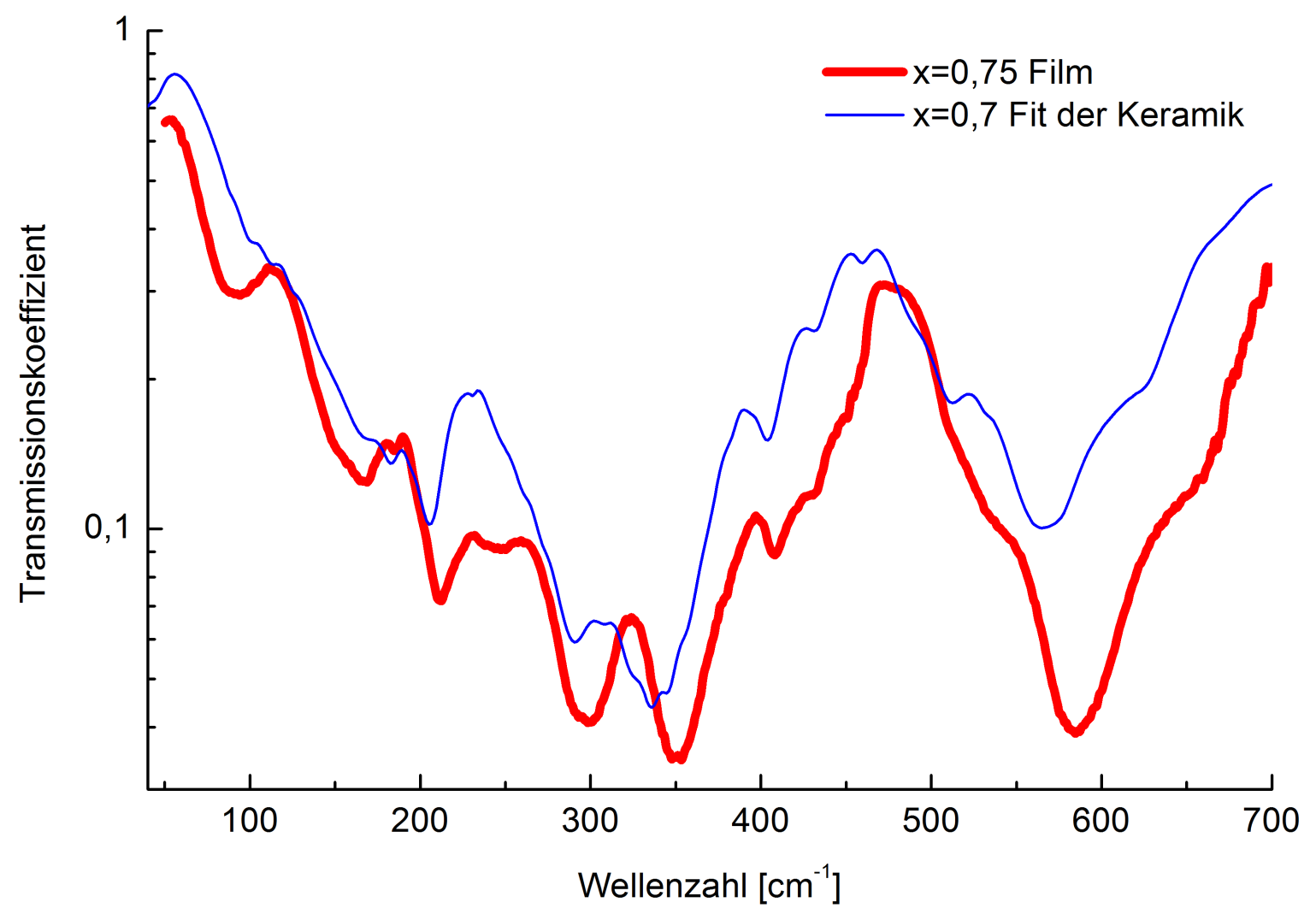

Abbildung 43 - FIR-Spektrum eines LCMO Films $x=0,75$ sowie der Fit der $x=0,7$ Keramik 


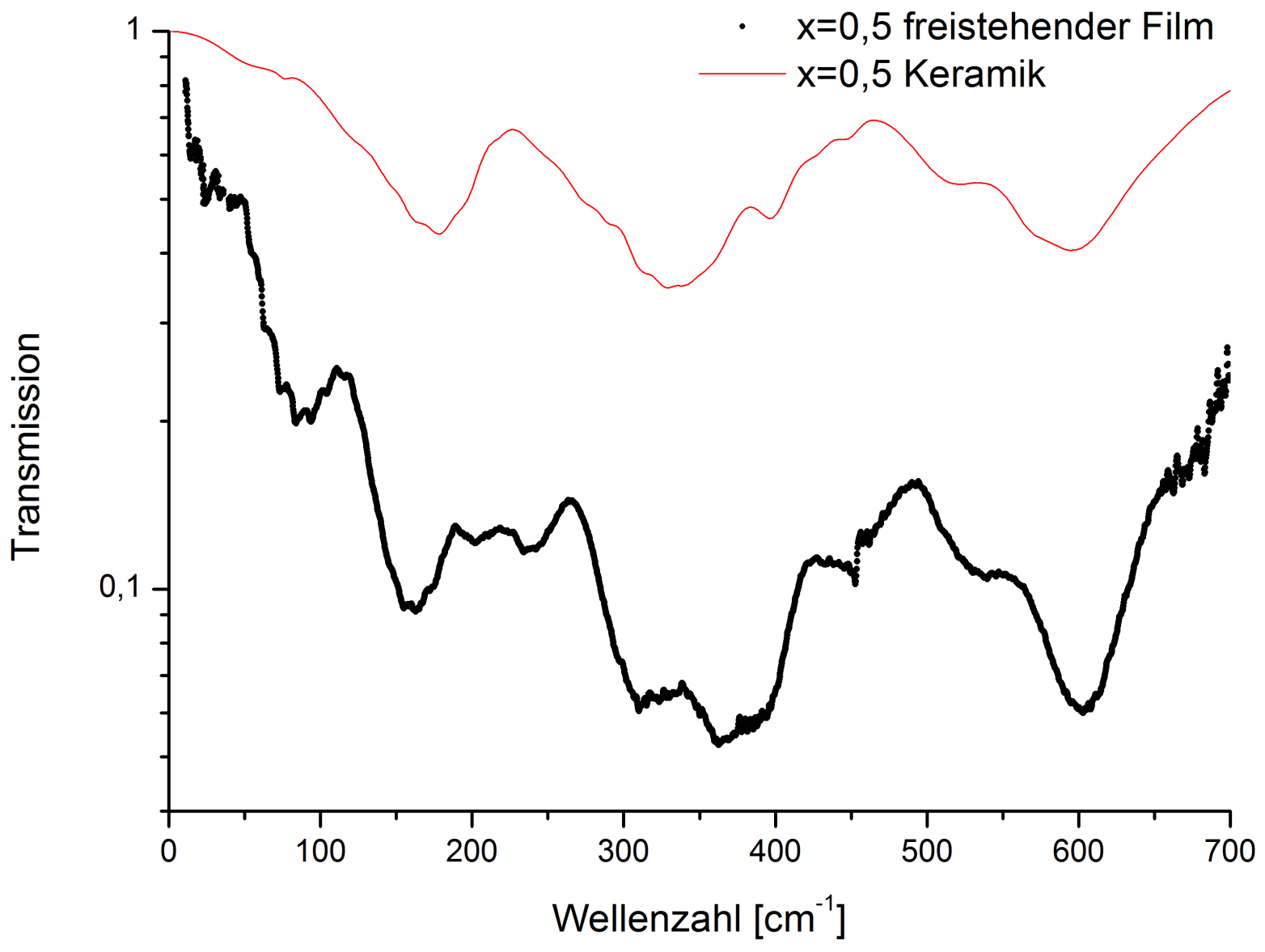

Abbildung 44 - FIR-Spektrum eines LCMO Films $x=0,5$ sowie der Fit der $x=0,5$ Keramik 


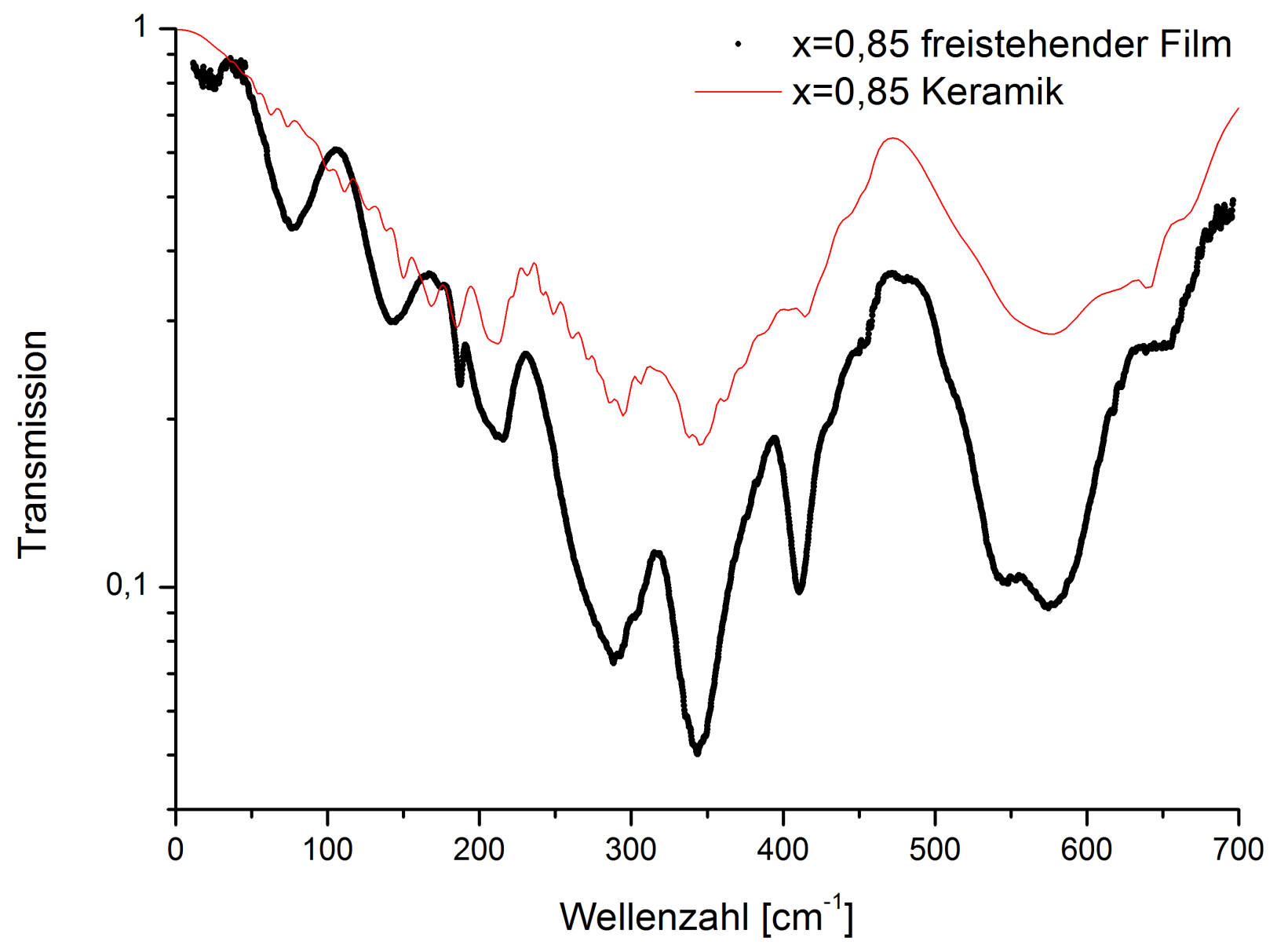

Abbildung 45 - FIR-Spektrum eines LCMO Films $x=0,85$ sowie der Fit der $x=0,85$ Keramik 
In Abb. 46 wird deutlich, dass das Modell für den Tesafilm, das für den Transfermatrixsansatz des Zweilagensystems Film+Tesa verwendet wird, noch verbessert werden muss. Der Unterschied wird besonders im Bereich unterhalb von $100 \mathrm{~cm}^{-1}$ deutlich. In diesem Bereich weicht der Fit stark von den wirklichen Werten der Transmissivität des Tesafilms ab und damit auch das subtrahierte Spektrum. Interessanterweise ist aber in diesem Bereich die Übereinstimmung mit den Keramiken erstaunlich hoch, obwohl die wirkliche Transmissivität der Filme deutlich unterhalb liegen sollte.

Ein weiteres Problem bei der Auswertung der Transmissionsmessungen sind Fabry-Perot-Interferenzen im Tesafilm, die eine weitere Auswertung der Kurven unterbindet. Damit sind die Spektren für Keramiken und freistehende Filme zwar ähnlich, aber doch nicht identisch. Auch unter der Annahme, dass die freistehenden Filme eine schlechtere kristalline Qualität aufweisen, als die auf $\mathrm{MgO}$ hergestellten Schichten, scheint es wahrscheinlich, dass als Einkristalle aufgewachsene freistehende Schichten ein verändertes Spektrum zeigen würden. Wobei der Unterschied sich hauptsächlich in der Schärfe der Absorptionslinien und im Bereich unterhalb von $100 \mathrm{~cm}^{-1}$ manifestieren sollte. Außerdem wichtig zu erwähnen ist die Tatsache, dass der Tesafilm eine Oszillation bei sehr kleinen Wellenzahlen zeigt. Solange der Fit dies nicht berücksichtigt ist es nicht möglich zu sagen, ob die Überstrukturpeaks auch für die freistehenden Filme aufgelöst werden können. Diese sollten invers mit der Intensität des Boson-Peaks skalieren. Je kleiner der Boson-Peak ist desto größer sollte die Intensität der „folded-phonons“ in der Leitfähigkeit sein.

Durch die Fabry-Perot Interferenzen können die Materialparameter der freistehenden Filme nicht berechnet werden und die Messungen beschränken sich damit auf das Aufzeigen der Infrarotmoden. Die im Weiteren durchgeführten Ramanmessungen stellen, aufgrund höherer Intensität und der Möglichkeit auch die Schichten auf $\mathrm{MgO}$ zu untersuchen, die bessere Alternative dar um Zugang zu den strukturellen Eigenschaften der Proben zu erhalten. 


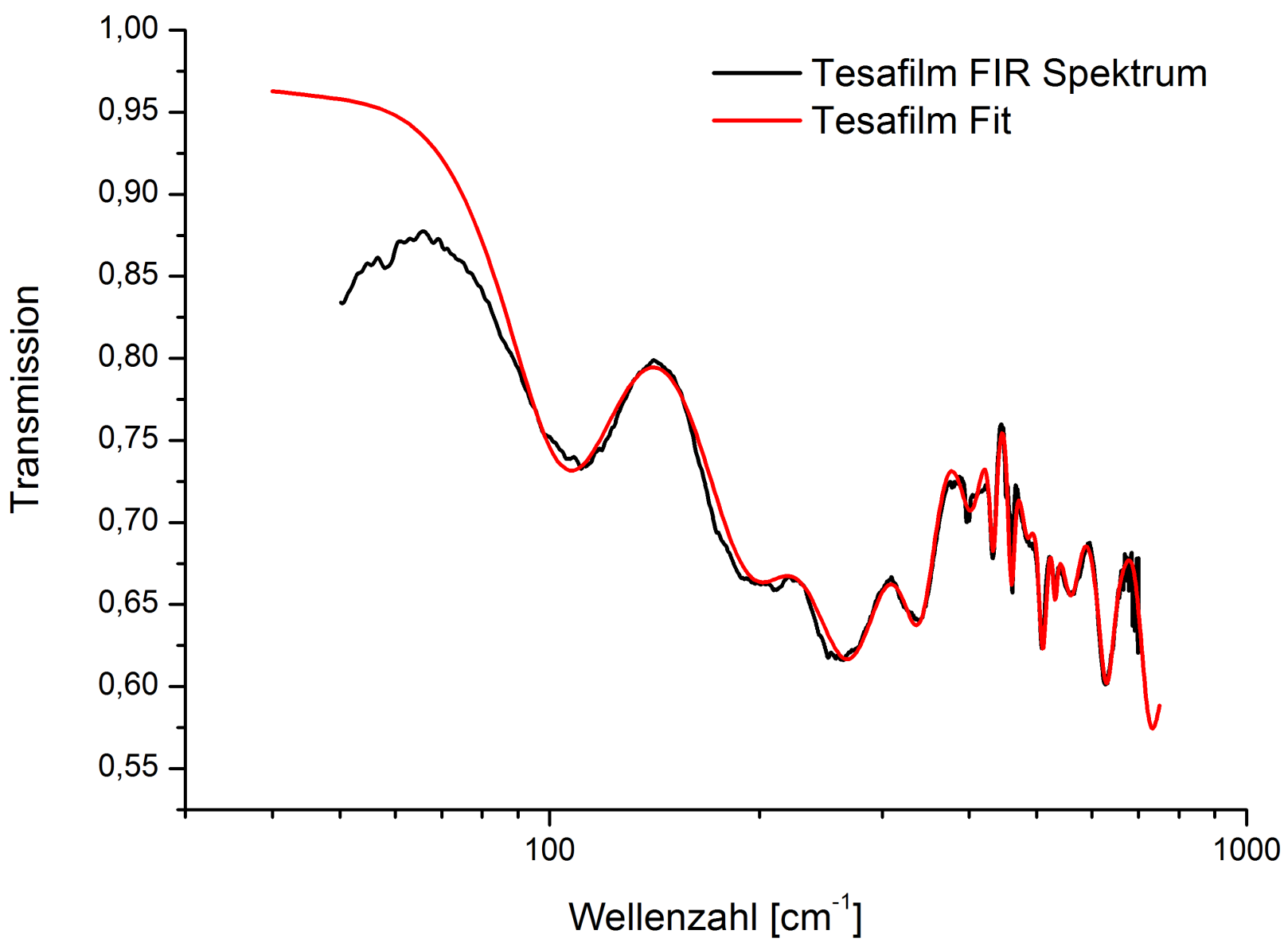

Abbildung $\mathbf{4} \boldsymbol{6}$ - FIR-Spektrum des verwendeten Tesafilms und zugehöriger Fit. Die deutliche Abweichung des Fits von den gemessenen Werten des Tesastreifens lässt eine Auswertung im Bereich $\omega<100 \mathrm{~cm}^{-1}$ unsinnig erscheinen 


\subsubsection{Ramanspektroskopie}

Die untersuchten überdotierten LCMO Proben weisen laut Literatur die orthorhombische Pnma Struktur auf [28]. Das bedeutet, nach Schönfliesnotation, sie gehören der $D_{2 h}$ Punktgruppe an. Für $D_{2 h}(\mathrm{mmm})$ existieren $7 A_{g}^{R S}+5 B_{1 g}^{R S}+7 B_{2 g}^{R S}+5 B_{3 g}^{R S}+8 A_{u}+9 B_{1 u}^{i r}+7 B_{2 u}^{i r}+9 B_{3 u}^{i r}$ Schwingungsmoden [82. Von diesen Moden können nur die ersten vierundzwanzig im Raman beobachtet werden. Die letzten fünfundzwanzig Moden sind IR-aktiv und damit vom Raman ausgeschlossen. Die $A_{u}$-Moden sind sogenannte stille Moden, die keine Intensität in Raman oder IR zeigen. Typischerweise können aber nur 10-12 Moden beobachtet werden [63]. Die Intensitätsverhältnisse der Moden zueinander, in den unterschiedlichen Konfigurationen, wurden im Appendix berechnet. Über die Intensitätsverhältnisse kann nun eine Zuordnung der Moden erfolgen 663, 83, 84. Bei den gemessenen Spektren wurde nach Abzug einer Basislinie (B-Spline), der Multipeak-Fit von Origin verwendet und Lorentzfunktionen angefittet. In vielen Veröffentlichungen ist die Basislinie kein B-Spline, sondern der sogenannte Bose-Einsteinfaktor [85. Dieser ist definiert über

$$
I_{e l}=\frac{\omega \frac{A}{\tau}}{\omega^{2}+\left(\frac{1}{\tau}\right)^{2}}+\frac{\omega \frac{A}{\tau}}{\omega^{2}+\left(\frac{1}{\tau}\right)^{2}} \cdot \frac{1}{1-\exp \left(\frac{-\hbar \omega}{k_{B} T}\right)}
$$

Dabei wird die Proportionalitätskonstante A, die Symmetrie abhängig ist und die Streurate der Elektronen $\tau$ für jede Konfiguration einzeln gefittet. Hierbei wird von kollisionsdominierten Ramanprozessen ausgegangen [86, 87]. Der Unterschied der beiden Basislinien, B-Spline zu Bose-Einstein, ist sehr gering, die Auswertung der Intensität des Ramansignals als $I_{g e s}=I_{p h}+$ $I_{e l}$ ist komplizierter. Daher wurde hier darauf verzichtet und die einfachere Basislinienfunktion von Origin verwendet. Der größte Fehler entsteht hier bei den sehr niedrigen Wellenzahlen, die bei der Basislinienfunktion von Origin typischerweise überkompensiert werden. In Abb. 47 sind die fünf Spektren der unterschiedlichen Dotierungen für die XX-Konfiguration zu sehen, die zur besseren Übersicht entlang der y-Achse versetzt wurden. In den Abb. 48 49 und 50 sind außerdem noch die X'X'-, X'Y'- und XY-Konfiguration zu sehen. 


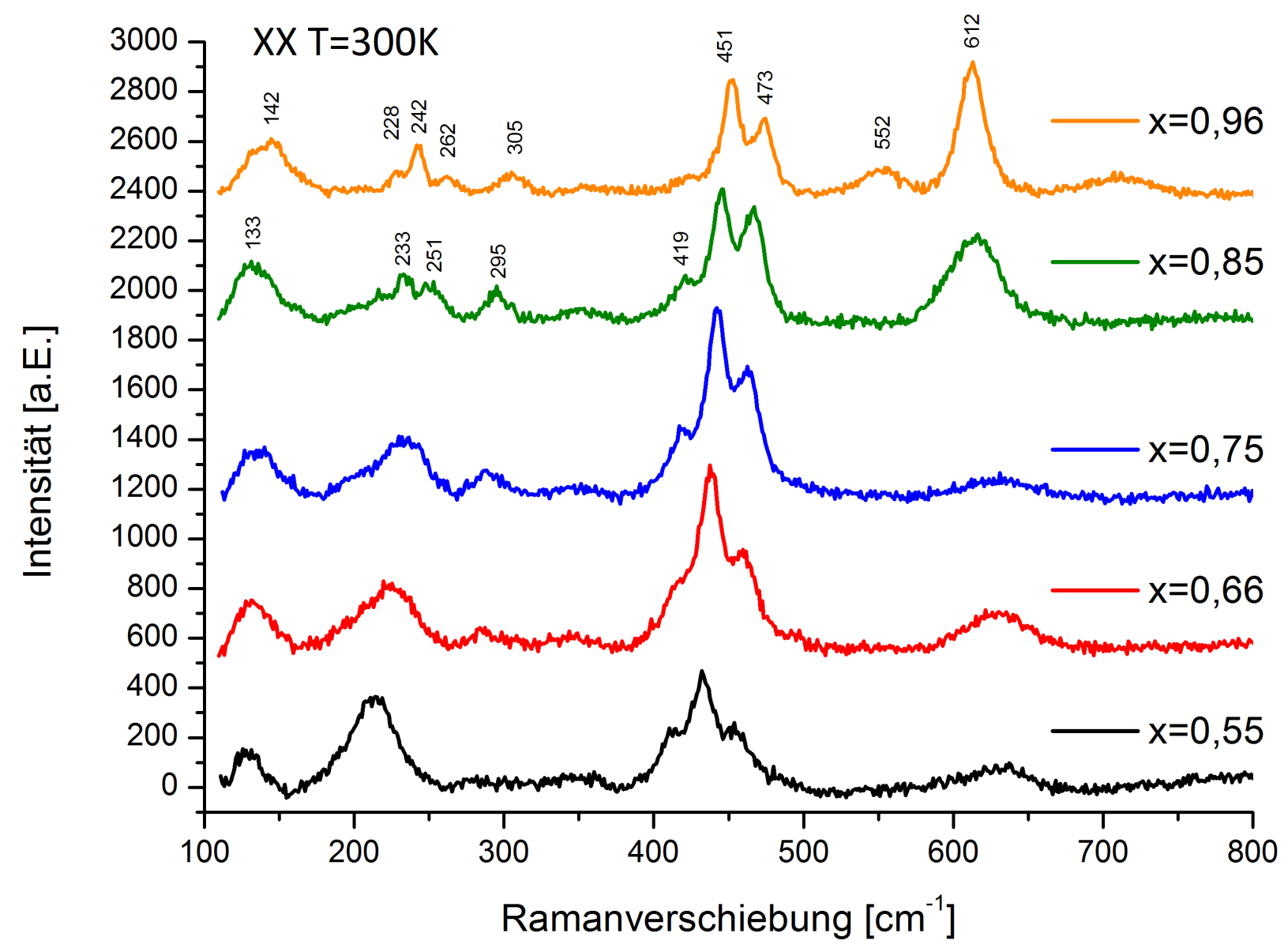

Abbildung 47-XX Konfiguration für alle Dotierungen 


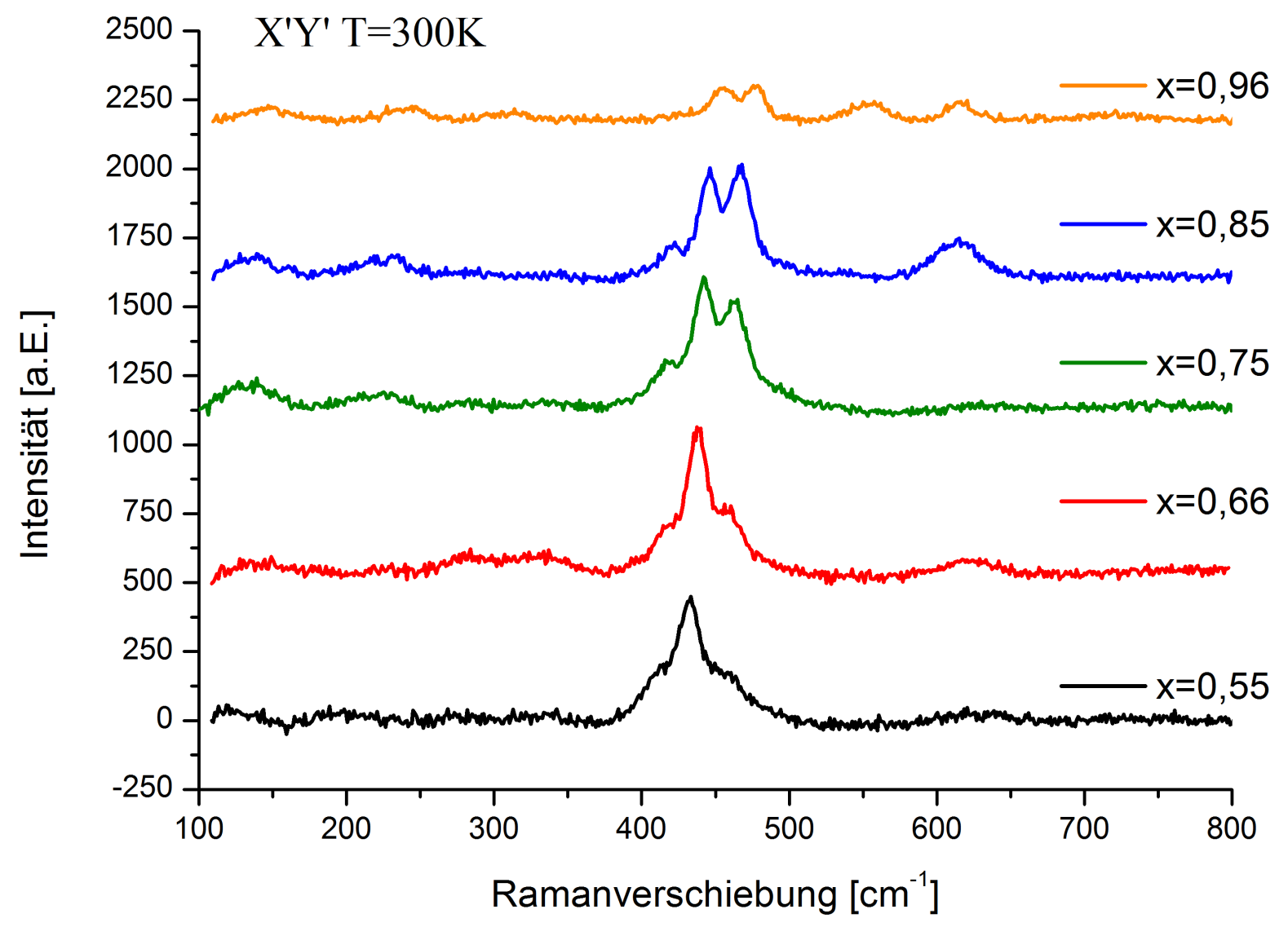

Abbildung $48-X^{\prime} Y^{\prime}$ Konfiguration für alle Dotierungen 


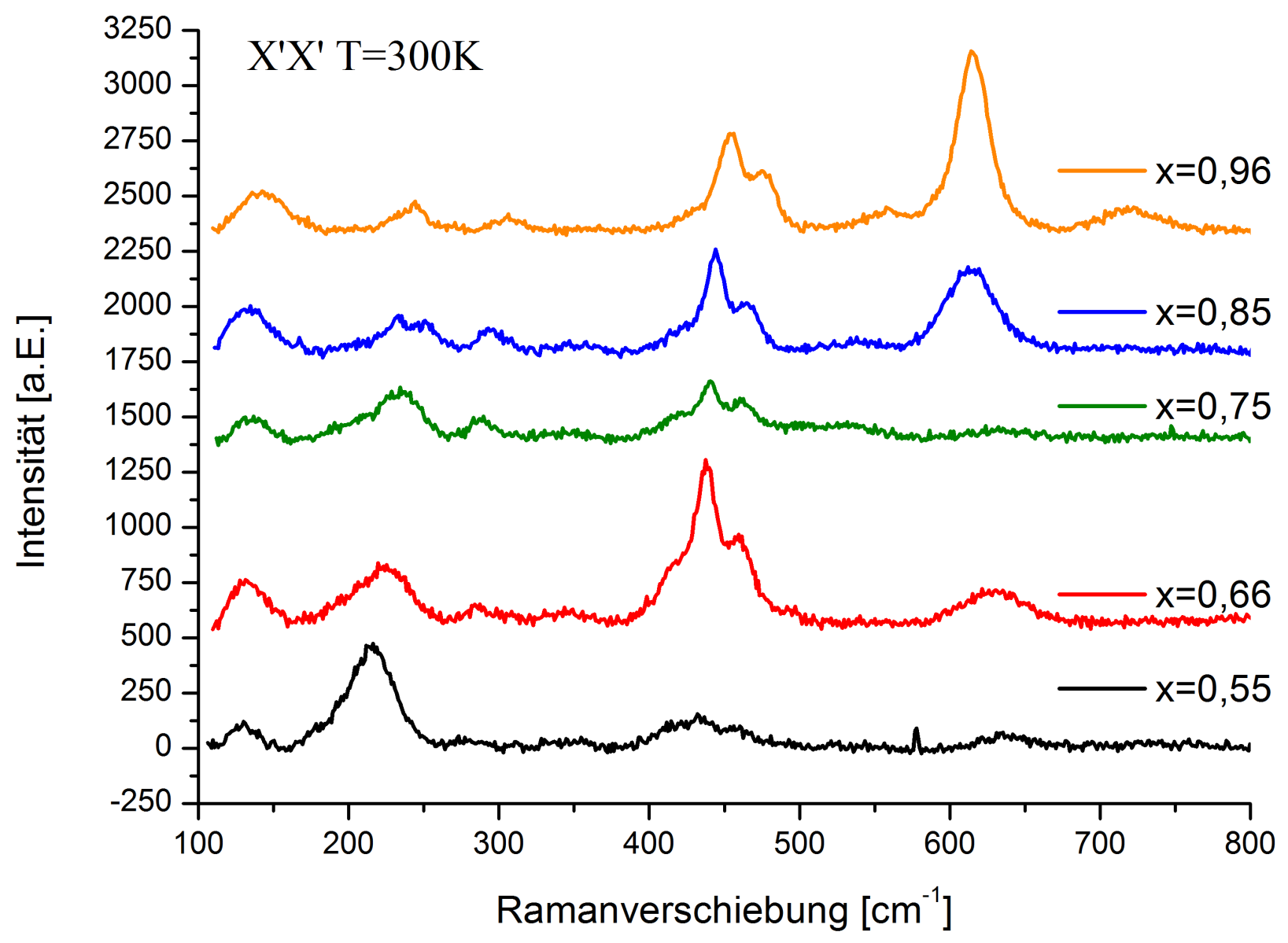

Abbildung $49-X^{\prime} X^{\prime}$ Konfiguration für alle Dotierungen 


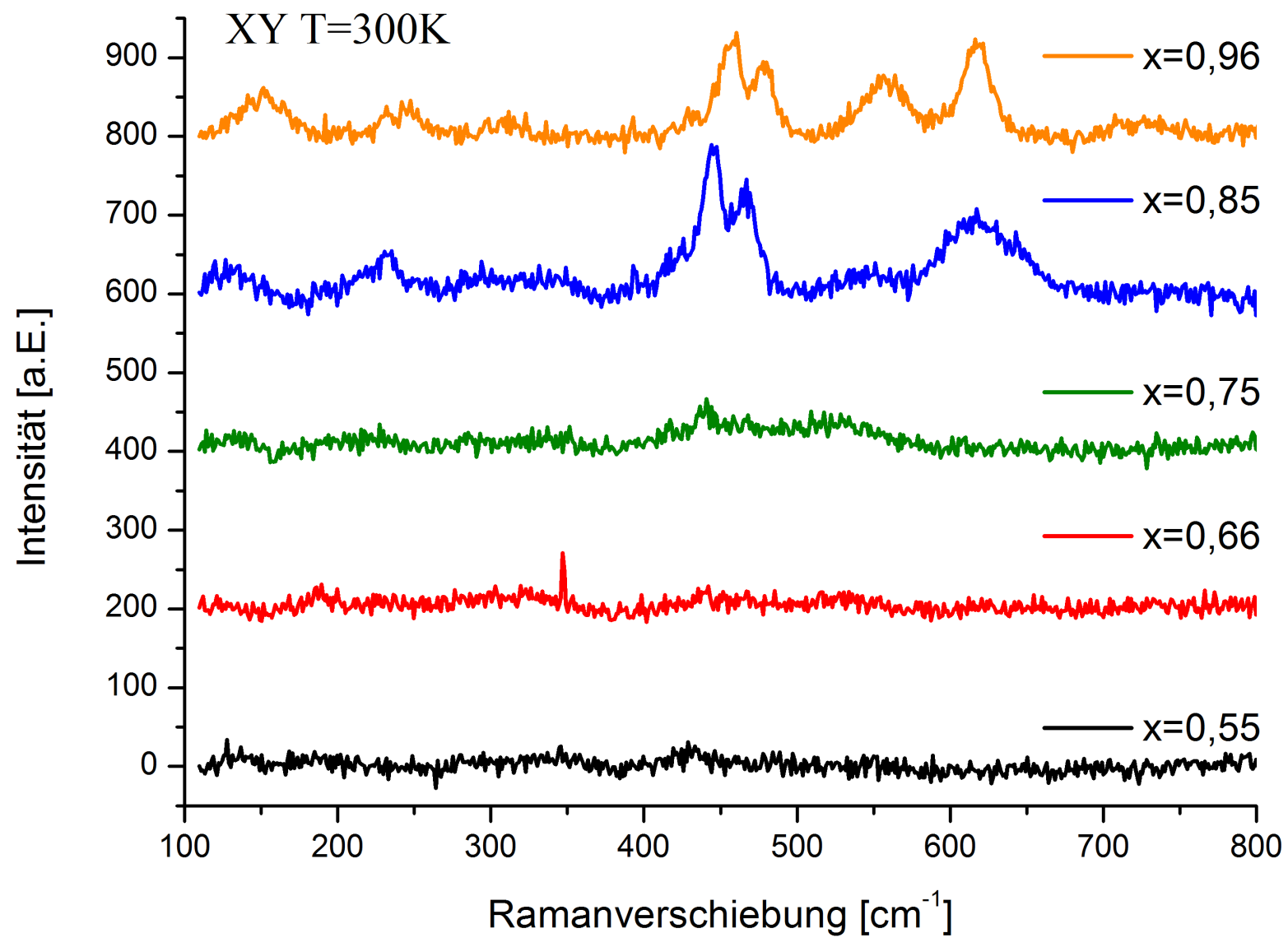

Abbildung 50 - XY Konfiguration für alle Dotierungen 
Die Intensitäten der einzelnen Moden werden nun für die vier Konfigurationen in die Tabellen 4, 5, 6, 77 und 8 eingetragen. Aus dem Verhältnis der Intensitäten zueinander kann nun anhand der Tabelle 2, für die Proben die entlang (001) aufwachsen, die Zuordnung der Moden erfolgen. Für die Proben die entlang (011) wachsen wird Tabelle 3 verwendet. Für die Dotierung $\mathrm{x}=0,85$ muss eine Mittlung der beiden Tabellen verwendet werden, da beide Orientierungen zu ähnlichen Teilen vorhanden sind. Die Spektren der fünf verschiedenen Dotierungen zeigen insbesondere im Bereich um $400-500 \mathrm{~cm}^{-1}$ die drei typischen starken $B_{2 g}$ Moden. Diese drei Moden werden in nahe$\mathrm{zu}$ allen Manganaten und auch vielen anderen Oxiden wie den Kupraten beobachtet 88 . Eine weitere starke Mode bei $600 \mathrm{~cm}^{-1}$ wird im Lanthanprototypen als Jahn-Teller-Mode identifiziert. Die schwächeren Moden bei $100-350 \mathrm{~cm}^{-1}$ können verschiedenen Ursprungs sein und treten besonders stark im ideal dotierten Bereich von LCMO auf [89].

\begin{tabular}{|c|c|c|c|c|}
\hline Mode & $\mathrm{XX}$ & $\mathrm{XY}$ & $\mathrm{X}^{\prime} \mathrm{X}^{\prime}$ & $\mathrm{X}^{\prime} \mathrm{Y}^{\prime}$ \\
\hline$B_{1 g}$ & 0 & 1 & 1 & 0 \\
\hline$B_{2 g}$ & 4 & 0 & 1 & 3 \\
\hline$B_{3 g}$ & 0 & 1 & 1 & 0 \\
\hline$A_{g}(a=b=c)$ & 6 & 0 & 6 & 0 \\
\hline$A_{g}(a=c \gg b)$ & 4 & 0 & 3 & 1 \\
\hline$A_{g}(a=c \ll b)$ & 2 & 0 & 1 & 1 \\
\hline
\end{tabular}

Tabelle 2 - Intensitätsverhältnisse für die Proben mit (001)-

Orientierung

\begin{tabular}{|c|c|c|c|c|}
\hline Mode & XX & XY & $\mathrm{X}^{\prime} \mathrm{X}^{\prime}$ & $\mathrm{X}^{\prime} \mathrm{Y}^{\prime}$ \\
\hline$B_{1 g}$ & 2 & 1 & 1,5 & 1,5 \\
\hline$B_{2 g}$ & 2 & 1 & 1,5 & 1,5 \\
\hline$B_{3 g}$ & 2,75 & 0,5 & 1,25 & 2,1875 \\
\hline$A_{g}(a=b=c)$ & 6 & 0 & 6 & 0 \\
\hline$A_{g}(a=c \gg b)$ & 2,75 & 0,625 & 2,935 & 0,0625 \\
\hline$A_{g}(a=c \ll b)$ & 0,75 & 0,625 & 0,936 & 0,0625 \\
\hline
\end{tabular}

Tabelle 3 - Intensitätsverhältnisse für die Proben mit (011)Orientierung 


\begin{tabular}{|c|c|c|c|c|c|}
\hline Shift $\left[\mathrm{cm}^{-1}\right]$ & XX [Counts] & X'Y' [Counts] $^{\prime}$ X'X' [Counts] & XY [Counts] & Symmetrie \\
\hline 128 & 144 & 0 & 97 & 0 & $A_{g}$ \\
\hline 212 & 381 & 0 & 472 & 0 & $A_{g}$ \\
\hline 413 & 153 & 103 & 68 & 0 & $B_{2 g}$ \\
\hline 432 & 406 & 400 & 110 & 0 & $B_{2 g}$ \\
\hline 456 & 169 & 113 & 64 & 0 & $B_{2 g}$ \\
\hline 630 & 84 & 0 & 54 & 0 & $A_{g}$ \\
\hline
\end{tabular}

Tabelle 4 - Intensitäten und Modenpositionen für $x=0,55$

\begin{tabular}{|c|c|c|c|c|c|}
\hline Shift $\left[\mathrm{cm}^{-1}\right]$ & XX [Counts] & X'Y' [Counts] $^{\prime}$ & $X^{\prime} X^{\prime}[$ Counts] & XY [Counts] & Symmetrie \\
\hline 132 & 198 & 0 & 117 & 0 & $A_{g}$ \\
\hline 222 & 224 & 0 & 205 & 0 & $A_{g}$ \\
\hline 288 & 56 & 53 & 31 & 0 & $B_{2 g}$ \\
\hline 342 & 0 & 54 & 34 & 0 & $B_{2 g}$ \\
\hline 415 & 168 & 98 & 75 & 0 & $B_{2 g}$ \\
\hline 437 & 653 & 492 & 98 & 0 & $B_{2 g}$ \\
\hline 461 & 292 & 161 & 118 & 0 & $B_{2 g}$ \\
\hline 515 & 0 & 0 & 117 & 54 & $B_{3 g}$ \\
\hline 630 & 152 & 49 & 62 & 0 & $B_{2 g}$ \\
\hline
\end{tabular}

Tabelle 5 - Intensitäten und Modenpositionen für $x=0,66$

\begin{tabular}{|c|c|c|c|c|c|}
\hline Shift $\left[\mathrm{cm}^{-1}\right]$ & XX [Counts] & X'Y' [Counts] $^{\prime}$ & $X^{\prime} X^{\prime}[$ Counts] & XY [Counts] & Symmetrie \\
\hline 135 & 195 & 86 & 92 & 0 & $B_{2 g}$ \\
\hline 207 & 52 & 0 & 54 & 0 & $A_{g}$ \\
\hline 230 & 233 & 47 & 208 & 0 & $B_{2 g}$ \\
\hline 290 & 86 & 0 & 78 & 0 & $A_{g}$ \\
\hline 417 & 183 & 110 & 75 & 0 & $B_{2 g}$ \\
\hline 441 & 674 & 384 & 211 & 0 & $B_{2 g}$ \\
\hline 463 & 423 & 331 & 128 & 0 & $B_{2 g}$ \\
\hline 520 & 0 & 0 & 54 & 55 & $B_{3 g}$ \\
\hline 630 & 72 & 0 & 33 & 0 & $A_{g}$ \\
\hline
\end{tabular}

Tabelle $\boldsymbol{6}$ - Intensitäten und Modenpositionen für $x=0,75$ 


\begin{tabular}{|c|c|c|c|c|c|}
\hline Shift $\left[\mathrm{cm}^{-1}\right]$ & XX [Counts] & $X^{\prime} Y^{\prime}[$ Counts] & $X^{\prime} X^{\prime}[$ Counts] & XY [Counts] & Symmetrie \\
\hline 133 & 247 & 73 & 203 & 29 & $A_{g}$ \\
\hline 214 & 81 & 0 & 0 & 0 & $A_{g}$ \\
\hline 233 & 142 & 68 & 132 & 43 & $B_{2 g}$ \\
\hline 251 & 126 & 0 & 96 & 0 & $B_{2 g}$ \\
\hline 295 & 121 & 0 & 96 & 0 & $B_{2 g}$ \\
\hline 350 & 52 & 0 & 0 & 0 & $A_{g}$ \\
\hline 419 & 125 & 78 & 56 & 26 & $A_{g}$ \\
\hline 444 & 477 & 324 & 421 & 173 & $A_{g}$ \\
\hline 466 & 420 & 363 & 172 & 110 & $A_{g}$ \\
\hline 630 & 370 & 136 & 390 & 95 & $A_{g}$ \\
\hline
\end{tabular}

Tabelle 7 - Intensitäten und Modenpositionen für $x=0,85$

\begin{tabular}{|c|c|c|c|c|c|}
\hline Shift $\left[\mathrm{cm}^{-1}\right]$ & XX [Counts] & $X^{\prime} Y^{\prime}[$ Counts] & $X^{\prime} X^{\prime}[$ Counts] & XY [Counts] & Symmetrie \\
\hline 142 & 220 & 50 & 200 & 54 & $A_{g}$ \\
\hline 228 & 67 & 0 & 0 & 0 & $B_{3 g}$ \\
\hline 242 & 189 & 48 & 121 & 32 & $B_{3 g}$ \\
\hline 262 & 57 & 0 & 0 & 0 & $B_{3 g}$ \\
\hline 305 & 76 & 29 & 63 & 0 & $B_{3 g}$ \\
\hline 451 & 433 & 112 & 426 & 118 & $B_{2 g}$ \\
\hline 473 & 257 & 112 & 198 & 78 & $B_{2 g}$ \\
\hline 552 & 93 & 66 & 71 & 72 & $B_{3 g}$ \\
\hline 612 & 535 & 69 & 844 & 122 & $A_{g}$ \\
\hline 712 & 71 & 25 & 110 & 19 & $A_{g}$ \\
\hline
\end{tabular}

Tabelle $\mathbf{8}$ - Intensitäten und Modenpositionen für $x=0,96$ 
In Abb. 51 sind ein Spektrum der XX-Konfiguration und der YY-Konfiguration zu erkennen. Da kein Unterschied zwischen den beiden Spektren besteht muss die Probe als verzwillingt angesehen werden. Im Weiteren ist in Abb. 52 die Auswertung Schritt für Schritt dargestellt.

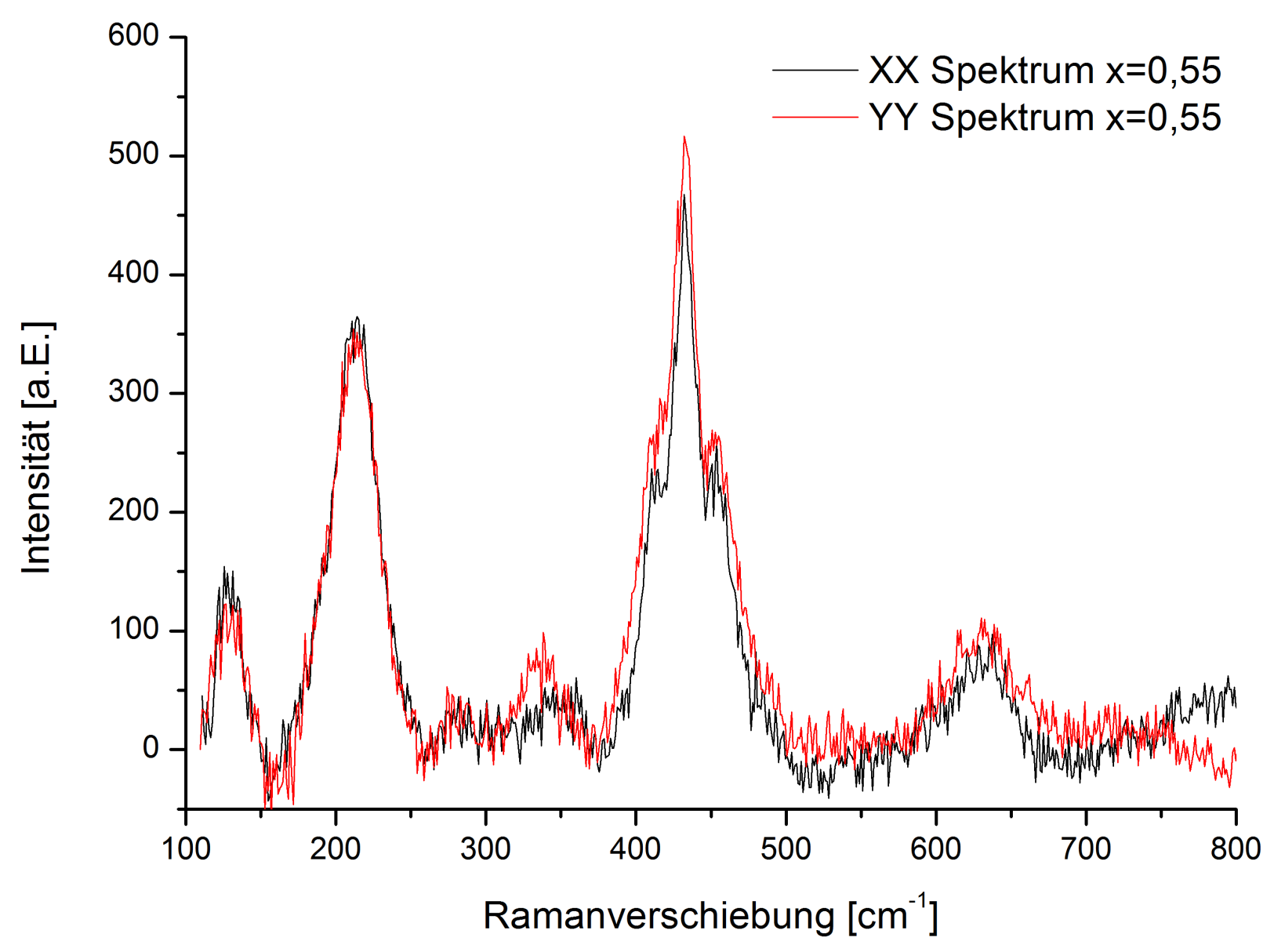

Abbildung 51 - Die Spektren der XX- und YY-Konfiguration weisen dieselben Intensitäten auf. 

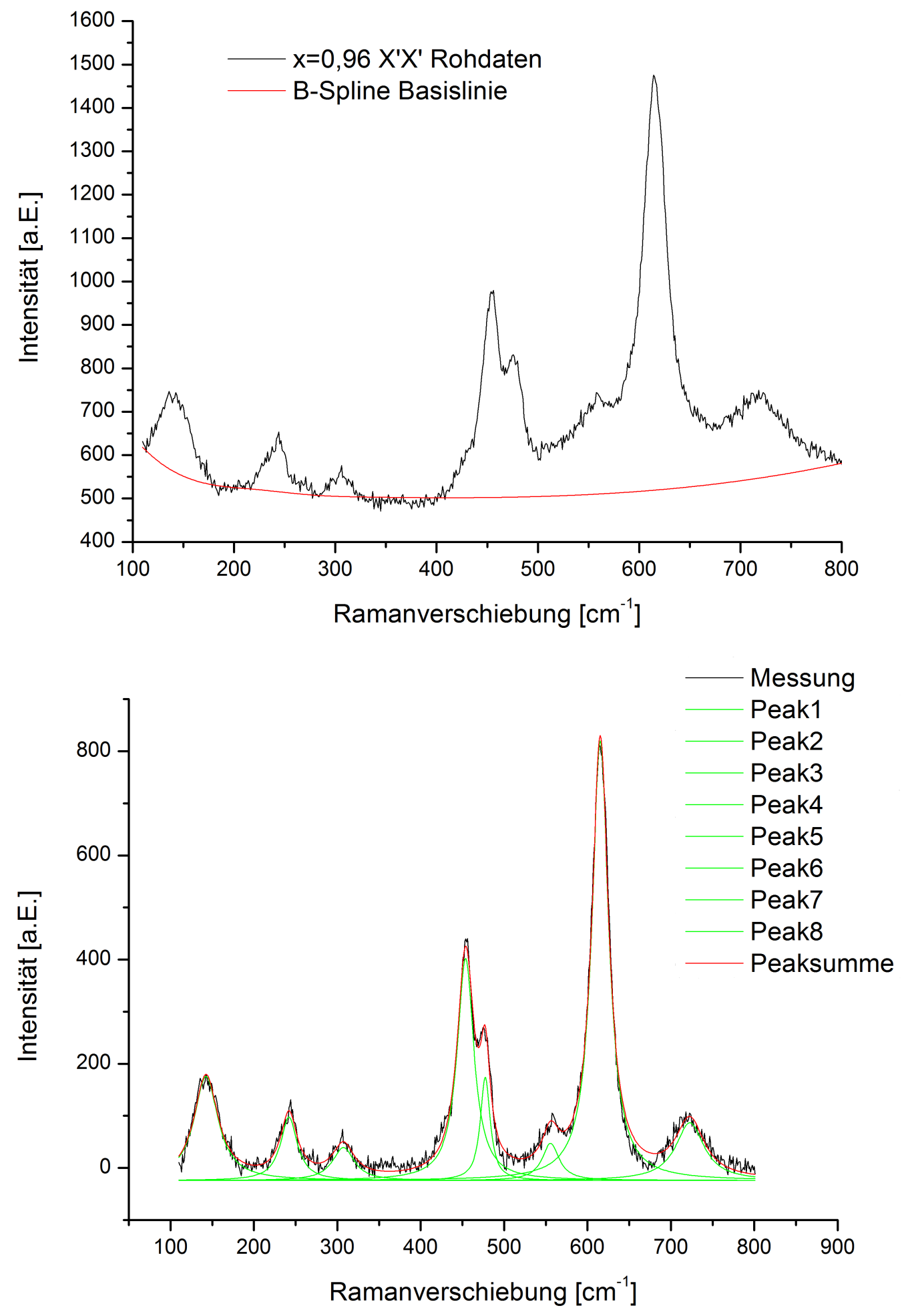

Abbildung 52 
Mithilfe von Sebastian Merten wurden ebenfalls temperaturabhängige Messungen durchgeführt. Da die Größe des maximalen Magnetfelds des Kryostaten zu gering ist um eine Änderung der Struktur herbeizuführen [27], sind nur temperaturabhängige Messungen der Proben mit $\mathrm{x}=0,75$ und $\mathrm{x}=0,96$ durchgeführt worden. Diese Messungen wurden zwei Monate nach den Ramanmessungen im normalen Mikroskopaufbau durchgeführt und können daher auch Aufschluss auf mögliche Änderungen der Proben an Luft geben. Es wurden Spektren für T=295, 250, 200, 150 und $50 \mathrm{~K}$ aufgenommen. Die Temperaturabhängigkeiten der vier Polarisationskonfigurationen sind in Abb. 54 , Abb. 55, Abb. 56 und Abb. 57 zu sehen. Hierbei wurden zuerst die XXKonfiguration und die XY-Konfiguration für alle Temperaturen, von $\mathrm{T}=295$ K abkühlend, vermessen. Danach wurde der Kryostat samt Probenraum aufgewärmt und die Probe neuerdings mit einem Winkel $45^{\circ}$ eingebaut. Daraufhin wurde wieder abgekühlt und die Konfigurationen X'X' und X'Y' vermessen. Die Spektren von $\mathrm{x}=0,96$ zeigen keine neuen oder verschwindenden Moden. Die Spektren von $\mathrm{x}=0,75$ zeigen jedoch, dass unterhalb der Ordnungstemperatur $T_{C O}$ zusätzliche Moden auftauchen und ein deutlicher Symmetriebruch sichtbar wird.

Nach dem anharmonischen Modell erwartet man weiter, dass die Position der Peaks sich unter Reduzierung der Temperatur zu höheren Wellenzahlen verschiebt nach

$$
\Delta(T)=C\left(1+\frac{2}{e^{\frac{\hbar \omega_{0}}{2 k_{B} T}}-1}\right)
$$

Hierbei ist $\Delta$ die temperaturabhängige Verschiebung der Position der Ramanmode, $\mathrm{C}$ eine Fitkonstante und $\omega_{0}$ die Wellenzahl bei $\mathrm{T}=0 \mathrm{~K}$. Wie in Abb. 53 zu sehen kann der Modenshift mit dem anharmonischen Modell gefittet werden, obwohl dies nur bis zur Ordnungstemperatur des Antiferromagneten möglich ist. Unterhalb der Ordnungstemperatur weicht die Modenposition deutlich ab. Eine Verringerung der Modenposition durch eine magnetische Ordnung ist dabei bereits in der Theorie in 90,91 beschrieben worden. 


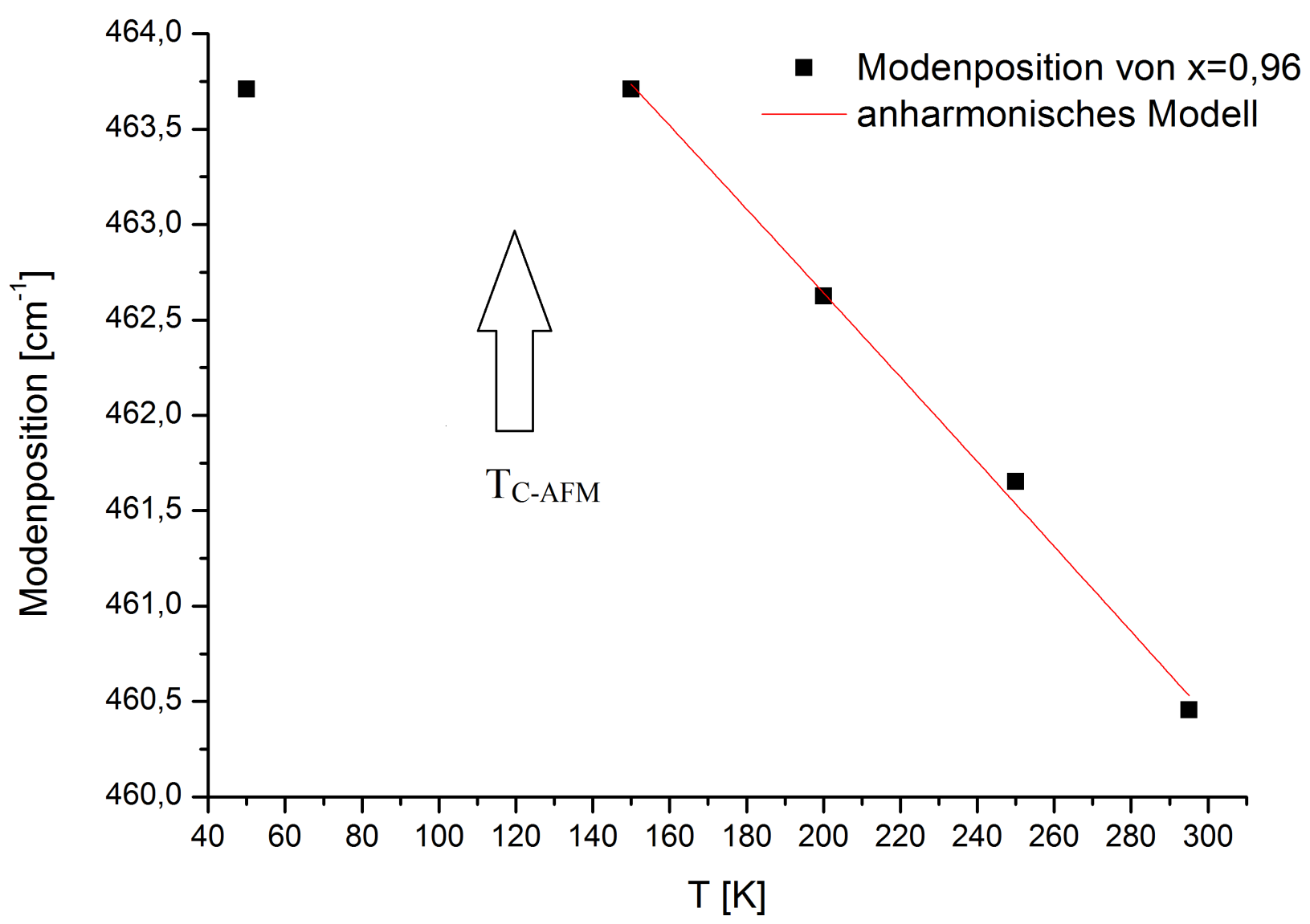

Abbildung 53 - Fit der Modenpositionen der $x=0,96$ Probe 
Die Intensitätsverhältnisse für $\mathrm{T}=50 \mathrm{~K}$ sind in Tabelle 9 dargestellt und zeigen, dass sich insbesondere die drei starken Moden bei $430-485 \mathrm{~cm}^{-1}$ nicht mehr nach Tabelle 3 richten.

\begin{tabular}{|c|c|c|c|c|c|}
\hline Shift $\left[\mathrm{cm}^{-1}\right]$ & XX [Counts] & X'Y' [Counts] $^{\prime}$ & $X^{\prime} X^{\prime}[$ Counts] & XY [Counts] & Symm. \\
\hline 148 & 432 & 155 & 273 & 92 & $B_{2 g}$ \\
\hline 161 & 662 & 307 & 586 & 134 & $A_{g}$ \\
\hline 239 & 157 & 98 & 159 & 50 & $B_{2 g}$ \\
\hline 255 & 398 & 163 & 354 & 100 & $B_{2 g}$ \\
\hline 277 & 198 & 0 & 168 & 0 & $A_{g}$ \\
\hline 324 & 277 & 60 & 288 & 63 & $A_{g}$ \\
\hline 435 & 297 & 132 & 231 & 201 & $B_{3 g}$ \\
\hline 462 & 2067 & 916 & 1536 & 450 & $B_{2 g}$ \\
\hline 485 & 1066 & 805 & 1137 & 263 & $B_{2 g}$ \\
\hline 574 & 200 & 157 & 204 & 249 & $B_{3 g}$ \\
\hline 625 & 2135 & 321 & 1495 & 214 & $A_{g}$ \\
\hline 736 & 794 & 164 & 578 & 106 & $A_{g}$ \\
\hline
\end{tabular}

Tabelle $\mathbf{9}$ - Dotierung $x=0,96$ bei $50 K$, Modenpositionen und Intensitäten

Aus den Abbildungen 54, 55, 56, und 57lässt sich folgern, dass die Proben mit $\mathrm{x}=0,96$ unterhalb von $150 \mathrm{~K}$ einen Übergang zeigen, da sich die Intensitätsverhältnisse bei tiefen Temperaturen ändern. Der Grund hierfür ist wahrscheinlich, die schon zuvor erwähnte, ferromagnetische Phasenseparation. Es wird deutlich, dass es unterhalb der C-AFM Temperatur eine zweite Phase gibt, die im Raman sichtbar wird und einen Beitrag zum Modenspektrum der $\mathrm{x}=0,96$ Probe besitzt. 


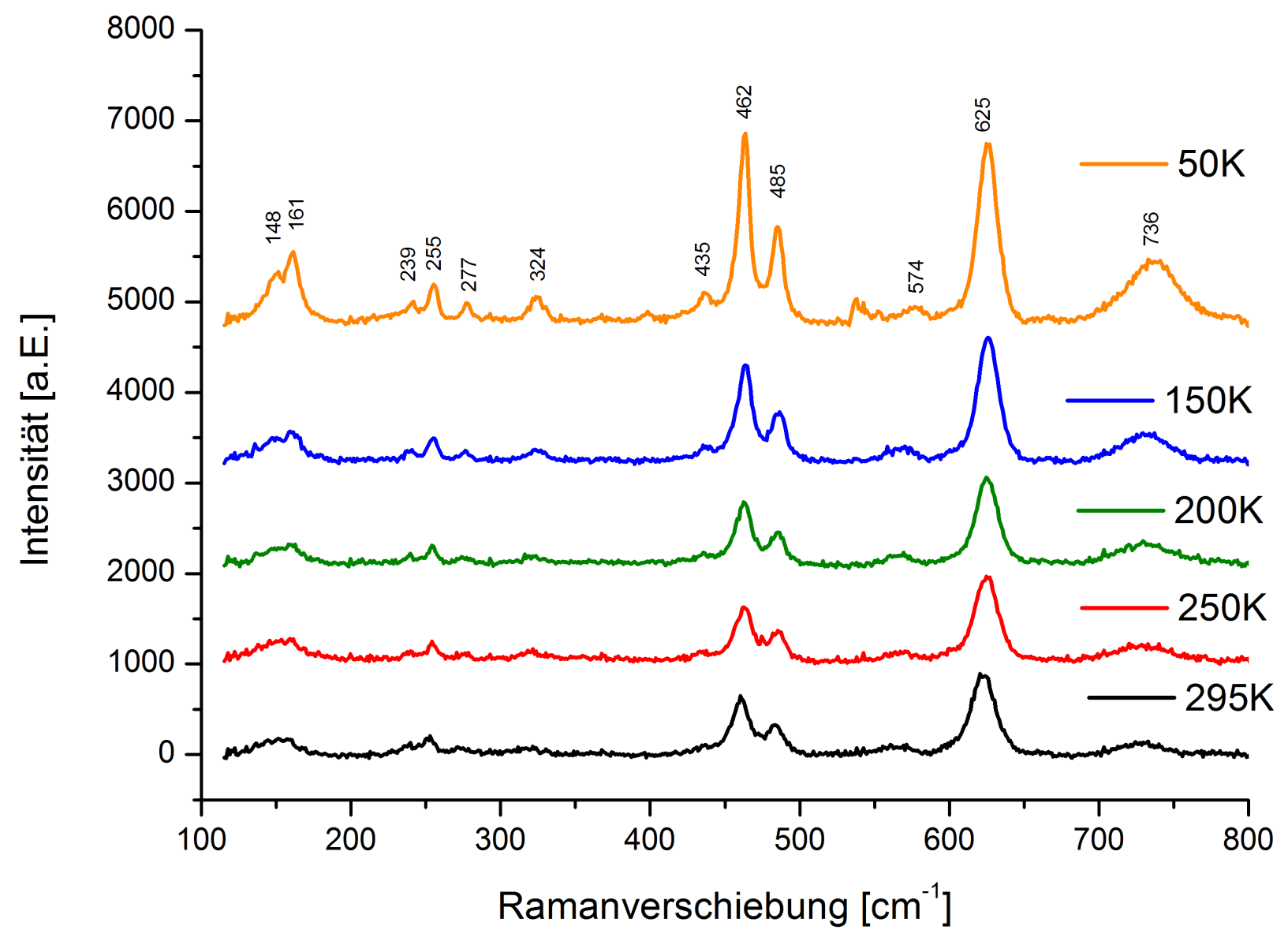

Abbildung $54-X X$ Konfiguration $x=0,96$ 


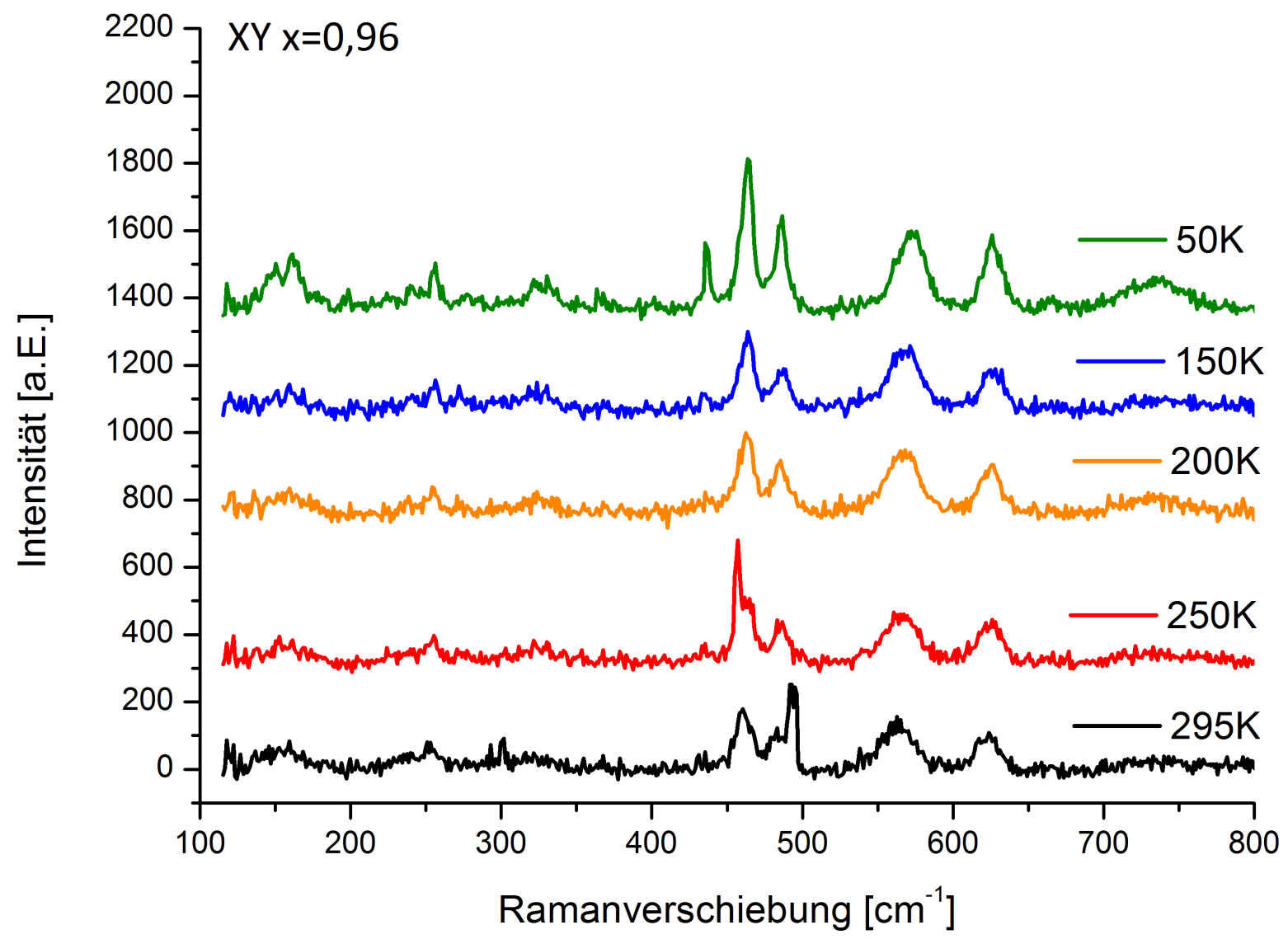

Abbildung $55-X Y$ Konfiguration $x=0,96$ 


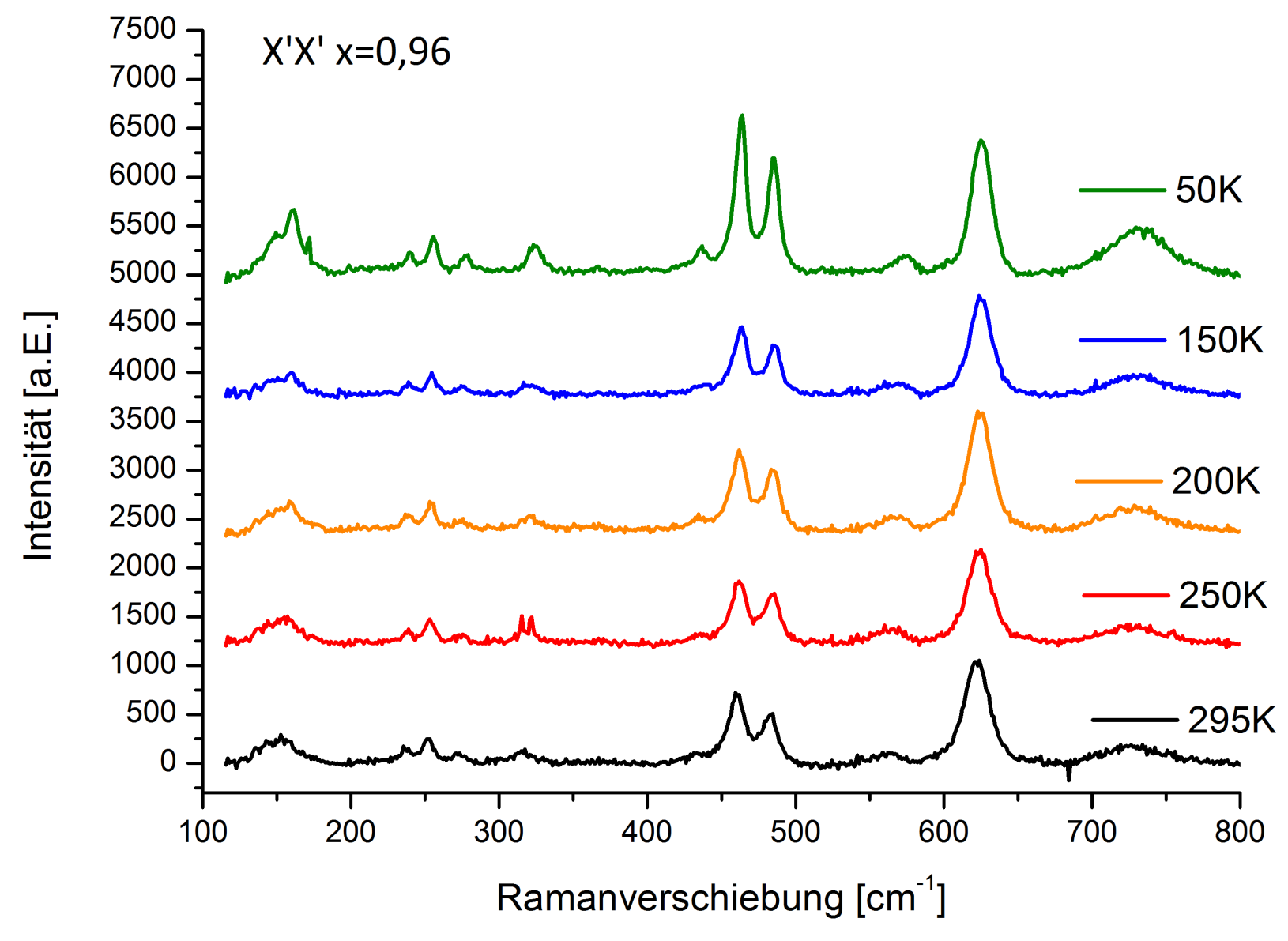

Abbildung $\mathbf{5 6}-X^{\prime} X^{\prime}$ Konfiguration $x=0,96$ 


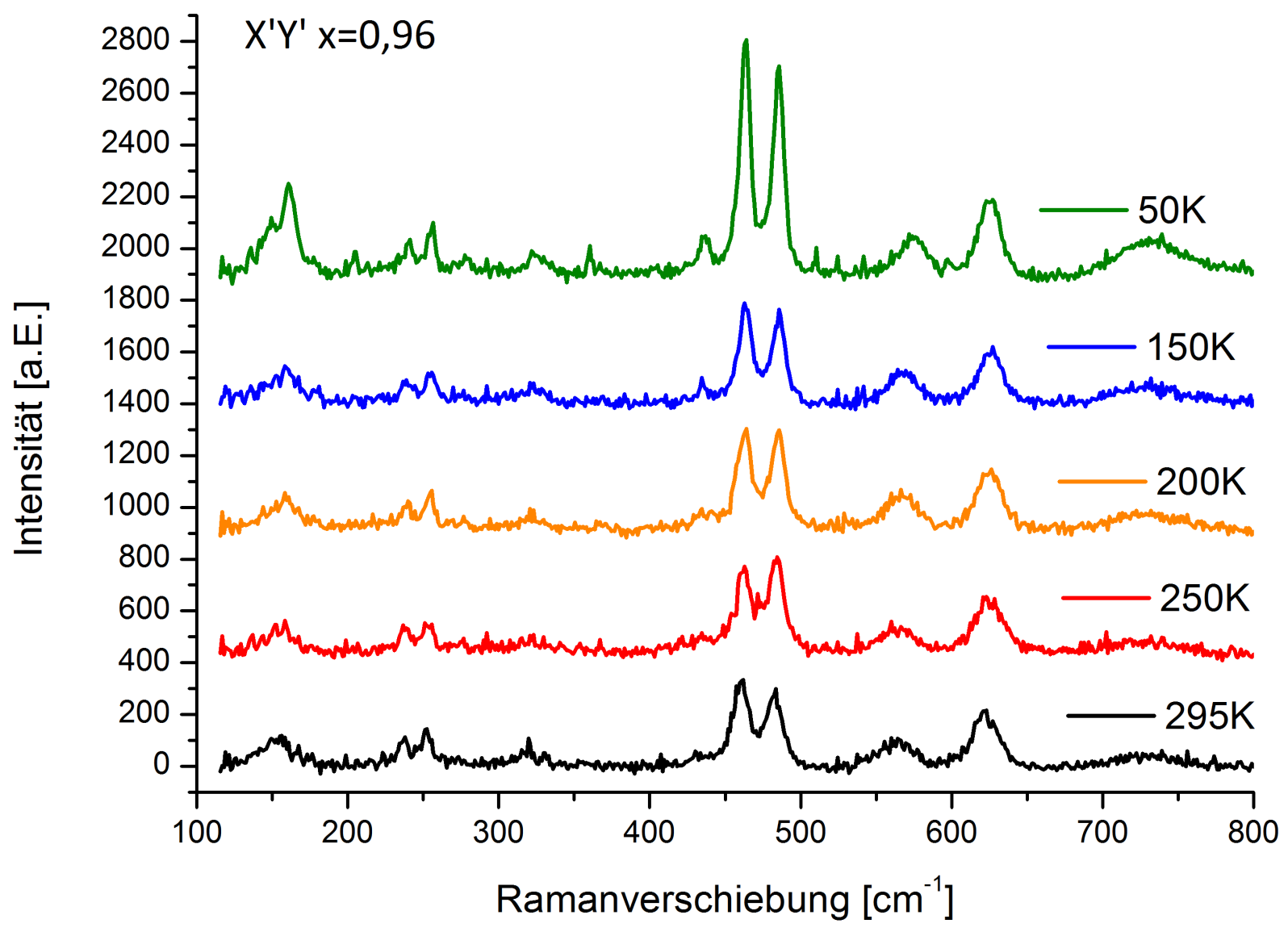

Abbildung $57-X^{\prime} Y^{\prime}$ Konfiguration $x=0,96$ 
Die Messungen für die $\mathrm{x}=0,75$ Probe wurden auf dieselbe Art und Weise durchgeführt wie die Messungen für $\mathrm{x}=0,96$. Zuerst wurden die Spektren für die Konfigurationen XX und XY gemessen, um nach erneutem Aufheizen auch $X^{\prime} X^{\prime}$ und X'Y' zu vermessen. Die daraus entstandenen Spektren sind in den Abbildungen 58, 59, 60 und 61 zu sehen. Anhand der Spektren wird deutlich, dass im Gegensatz $\mathrm{zu} x=0,96$ die Probe mit Dotierung $\mathrm{x}=0,75$ einen Symmetriebruch unterhalb $\mathrm{T}=200 \mathrm{~K}$ zeigt. Eindeutige Indizien sind hierbei: - Die neu auftretenden starken Moden bei $646 \mathrm{~cm}^{-1}$ und $707 \mathrm{~cm}^{-1}$. Insbesondere die Mode bei $707 \mathrm{~cm}^{-1}$ ist oberhalb von $\mathrm{T}=150 \mathrm{~K}$ nicht vorhanden, sowie die starke Intensität mit der sie das erste Mal unterhalb der Ladungsordnungstemperatur auftritt lässt auf einen Symmetriebruch schließen.

- Die hohe Intensität der $B_{g}$-Moden in der XY-Konfiguration. Aufgrund der Änderung der Intensitätsverhältnisse von Tab. 2 zu Tab. 10 wird deutlich, dass die $B_{g}$-Moden nun eine nicht verschwindende Intensität auch in der XYKonfiguration besitzen. Die starke Intensitätsänderung kann nicht mit dem normalen Temperatureffekt und einer schlechten Ausrichtung erklärt werden.

-Das sich die Spektren der X'Y'-Konfiguration, außer durch die zusätzlich auftauchenden Moden, nicht ändern bestätigt, dass die theoretisch berechneten Tabellen die Intensitätsverhältnisse korrekt wiedergeben, da auch hier die X'Y'-Konfiguration in der Intensität unverändert ist.

Nicht ausgeschlossen werden kann jedoch, dass sich die Koeffizienten der Ramantensoren nach dem Symmetriebruch ändern.

Damit ist es möglich durch temperaturabhängige Ramanmessungen den Symmetriebruch der Probe unterhalb von $\mathrm{T}=200 \mathrm{~K}$ sichtbar zu machen. Eine Zuordnung der Moden, unterhalb von $\mathrm{T}=200 \mathrm{~K}$, ist aufgrund der erhöhten Anzahl an Matrixelementen nicht mehr möglich, da zu viele Annahmen gemacht werden müssten, als das eine verlässliche Zuordnung möglich wäre. Ähnliche Messungen scheiterten bisher an der Kristallqualität 92.

\begin{tabular}{|c|c|c|}
\hline Konf. & $A_{g}$ & $B_{g}$ \\
\hline XX & $\frac{1}{6}\left((a+c)^{2}+2 b^{2}\right)$ & $\frac{2}{3} e^{2}$ \\
\hline XY & $\frac{1}{6}\left[\frac{1}{2}(a-c)^{2}+2 d^{2}\right]$ & $\frac{1}{3}\left(f^{2}\right)$ \\
\hline$X^{\prime} X^{\prime}$ & $\frac{1}{6}\left[\frac{1}{4}(a+2 b+c+2 \sqrt{2} d)^{2}+a^{2}+c^{2}\right]$ & $\frac{1}{6}\left(e^{2}+2 f^{2}\right)$ \\
\hline$X^{\prime} Y^{\prime}$ & $\frac{1}{24}(-a+2 b-c)^{2}$ & $\frac{1}{2}\left(e^{2}\right)$ \\
\hline
\end{tabular}

Tabelle 10 - Intensitätsverhältnisse der P2 $/ / m$-Struktur 


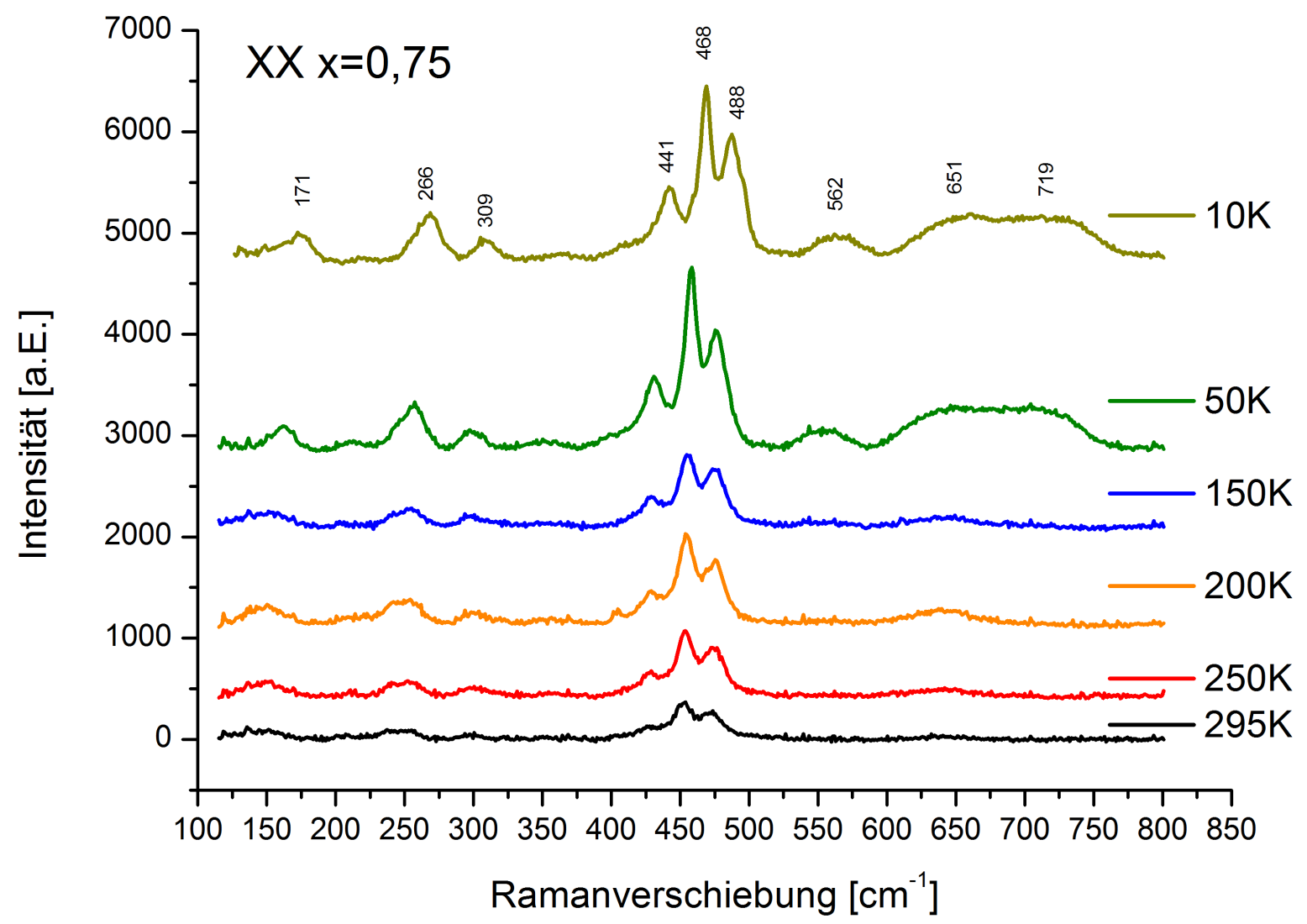

Abbildung 58 - XX Konfiguration der Probe mit Dotierung x=0,75. Unterhalb von $T=200 \mathrm{~K}$ werden zusätzliche Moden sichtbar bei $646 \mathrm{~cm}^{-1}$ und $707 \mathrm{~cm}^{-1}$ 


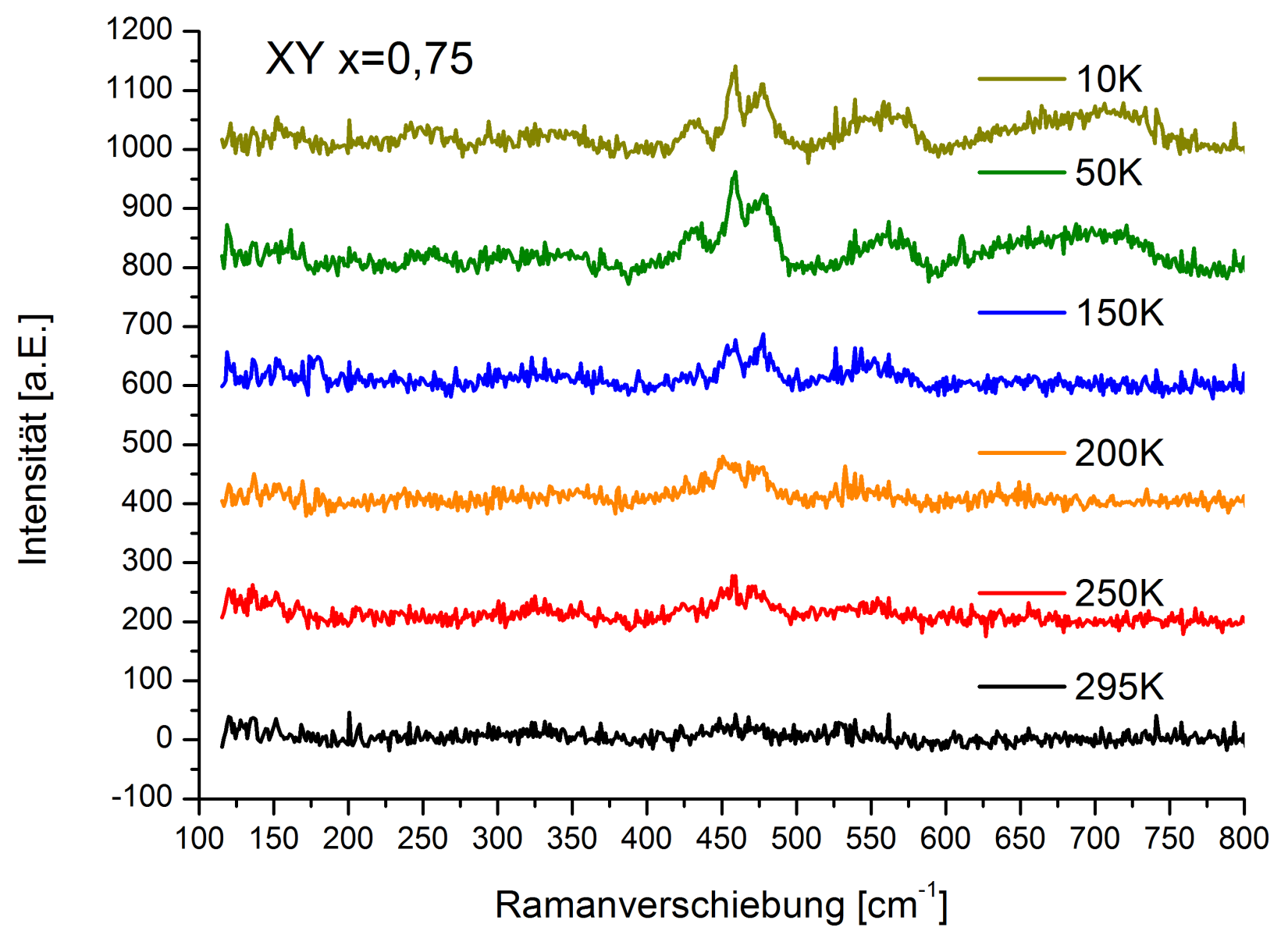

Abbildung 59 - XY Konfiguration der Probe mit Dotierung $x=0,75$. Unterhalb von $T=200 \mathrm{~K}$ haben die B-Moden spektrales Gewicht und werden sichtbar 


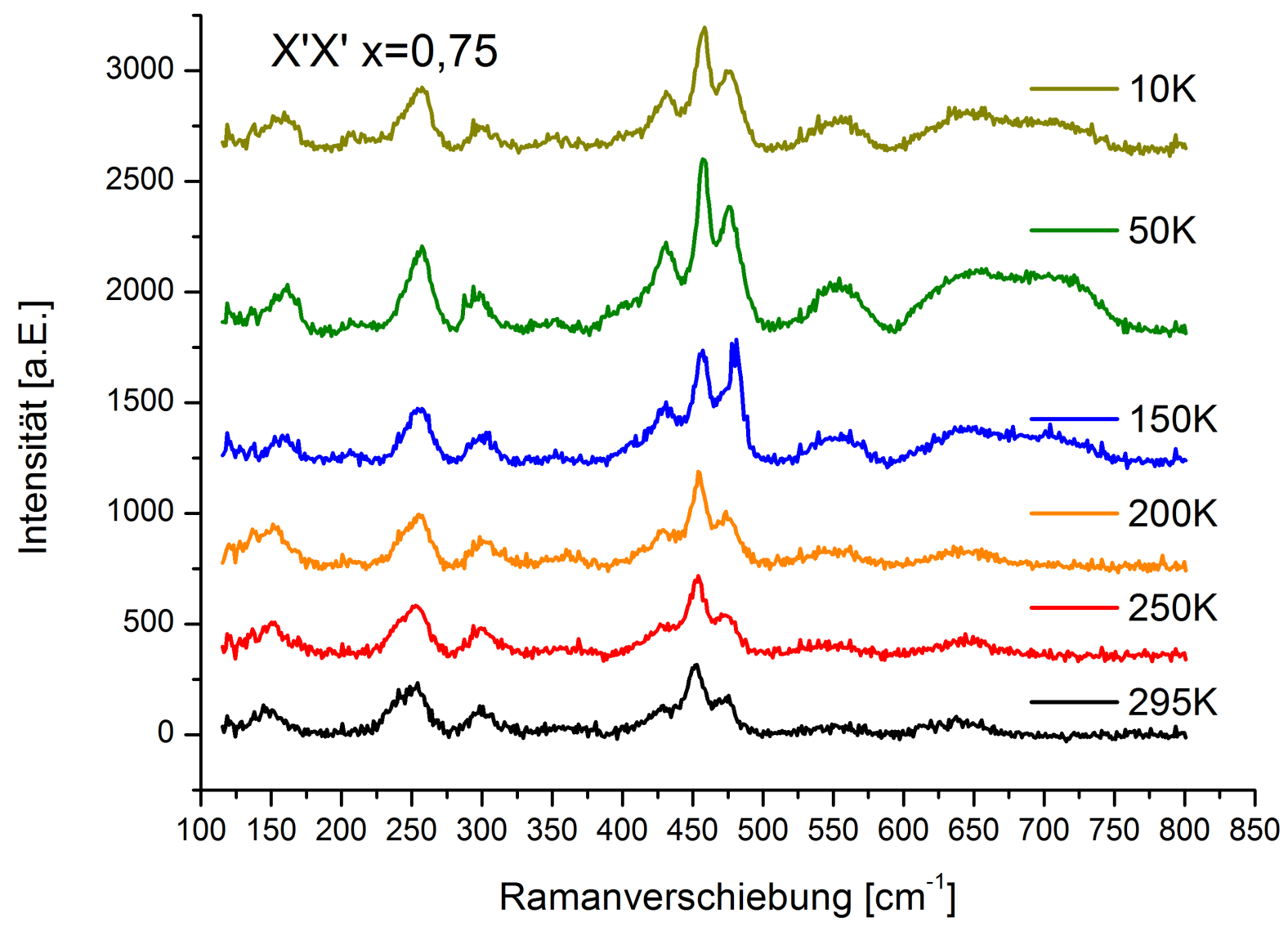

Abbildung $60-X^{\prime} X^{\prime}$ Konfiguration der Probe mit Dotierung $x=0,75$. Unterhalb von $T=200 \mathrm{~K}$ werden zusätzliche Moden sichtbar bei $646 \mathrm{~cm}^{-1}$ und $707 \mathrm{~cm}^{-1}$ 


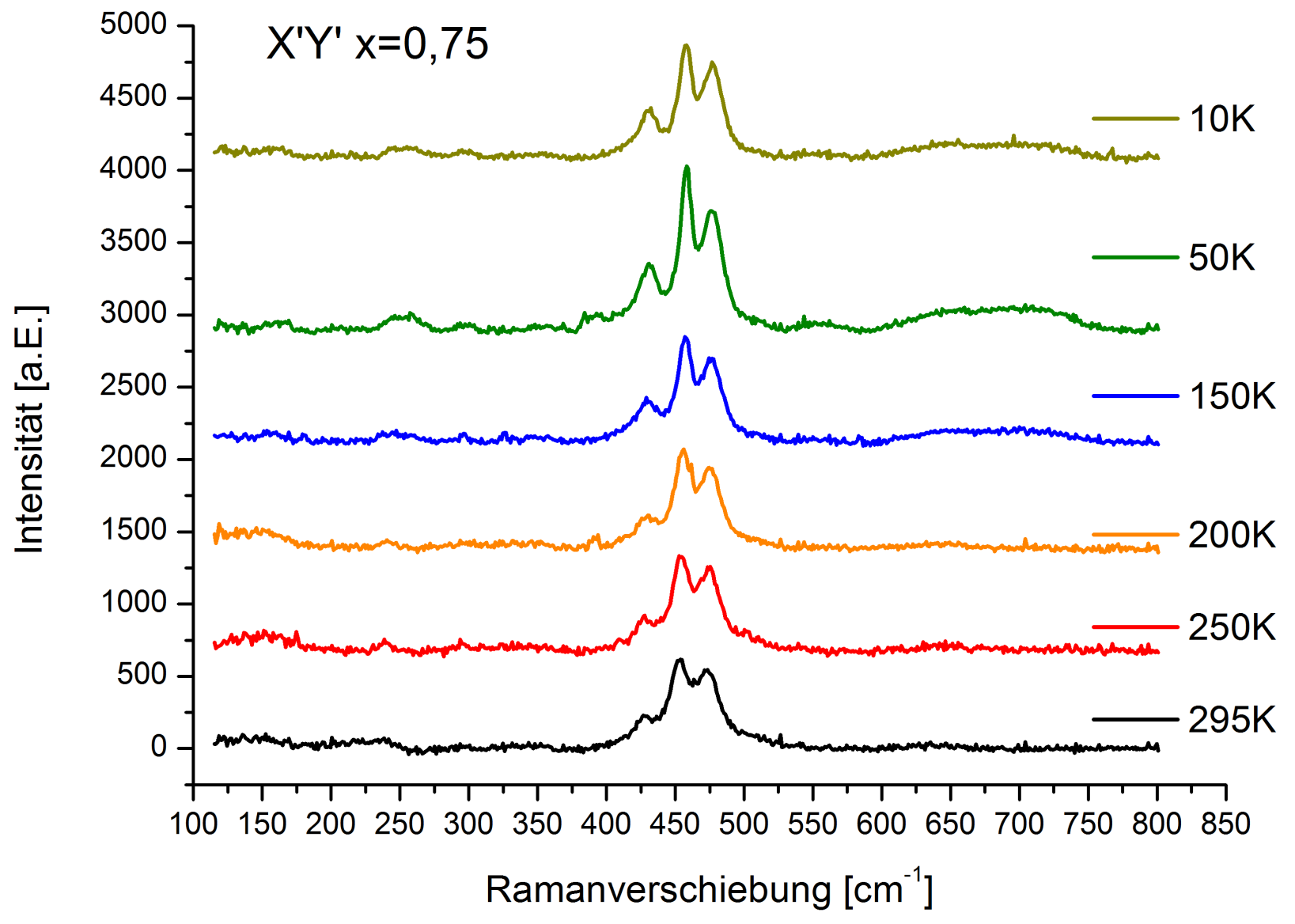

Abbildung $61-X^{\prime} Y^{\prime}$ Konfiguration der Probe mit Dotierung $x=0,75$. Es ist außer den zusätzlichen Moden keine Änderung der Intensitäten zu erkennen 


\subsubsection{TERS}

Die überdotierten LCMO Proben lassen sich ebenfalls für TERS verwenden, da insbesondere die überdotierten Proben eine Mode besitzen, die sehr hohe Intensität zeigt bei $630 \mathrm{~cm}^{-1}$. Dies ist nötig um den richtigen Fokus des Laserspots zu finden und somit die TERS-Messung erst möglich zu machen. Die LCMO Schichten waren außerdem die ersten Manganatproben, die mit dem TERS erfolgreich untersucht wurden. Mithilfe von Christoph Meyer wurden TERS-Messungen an der Probe mit $\mathrm{x}=0,96$ durchgeführt. Die Ergebnisse dieser Messungen sollen hier kurz präsentiert werden. In Abb. 62 ist das Spektrum mit angenäherter Spitze und das Spektrum ohne Spitze zu sehen, sowie nach Verschiebung des Laserspots. Die TERS-Verstärkung wird besonders an den Moden bei $440 \mathrm{~cm}^{-1}$ und $630 \mathrm{~cm}^{-1}$ deutlich. Abhängig von der Position der Spitze und des Lasers war es nun möglich ein unterschiedliches Modenspektrum und eine sehr starke Mode bei $646 \mathrm{~cm}^{-1}$ in der Probe mit $\mathrm{x}=0,96 \mathrm{zu}$ finden. Diese Mode, die bei $646 \mathrm{~cm}^{-1}$ liegt, tritt nur in sehr begrenzten Gebieten nach Verschiebung des Laserspots auf und lässt sich möglicherweise mit kleinsten Verunreinigungen der Oberfläche erklären. Ein möglicher Kandidat wäre dabei das Hausmannit [93. Hausmannit besitzt eine starke Ramanmode in der Nähe von $640 \mathrm{~cm}^{-} 1$, die sich mit der Größe der Kristallite verschiebt. Laut Zuo Liu und Qian würde die Größe des Hausmannit 32nm großen Kristalliten entsprechen [93. Diese zusätzlichen Moden und ihre starke Abhängigkeit von der Laser- und Spitzenposition zeigen den Vorteil von TERS, der hohen Ortsselektivität. Die überdotierten Manganate eignen sich dabei gut für TERS Aufnahmen, da die anfängliche, meist schwierige Konfiguration des Setups durch einige relativ starke Moden erleichtert wird. 


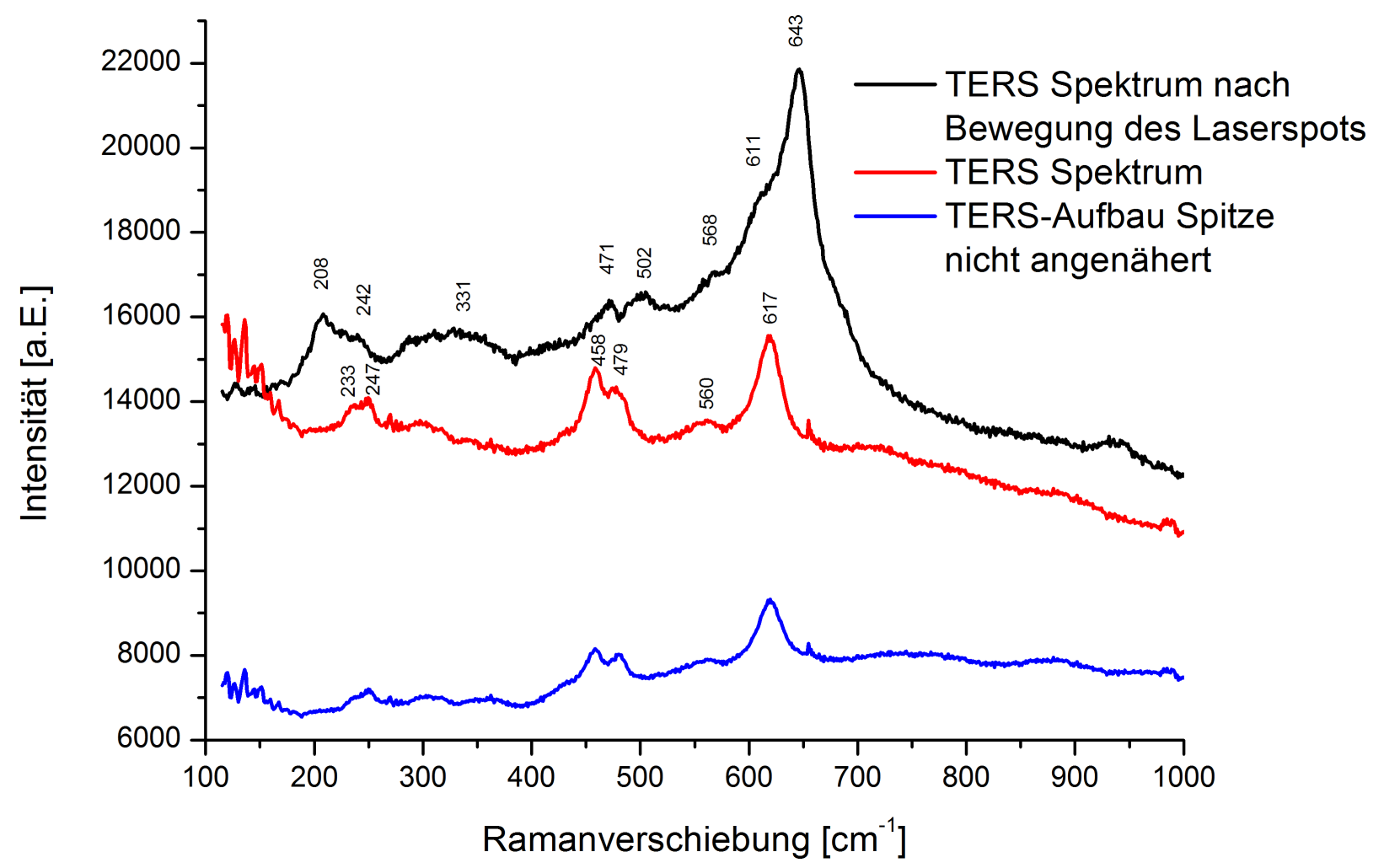

Abbildung 62 - TERS-Spektrum des LCMO Films mit $x=0,96$ bei angenäherter Spitze, ohne Annäherung und nach Verschiebung der Laserposition um einige $\mu m$ 


\section{Zusammenfassung und Diskussion}

Die hergestellten Proben insbesondere die freistehenden Filme sind die Ersten dieser Art. Die Untersuchungen haben gezeigt, dass viele der Eigenschaften von Polykristallen reproduziert werden konnten. Eine der wenigen Eigenschaften, die nicht reproduziert werden konnte, ist die Ladungsordnung die als Plateau im Widerstand sichtbar wird. Auch die globalen Messungen der Leitfähigkeit zeigen, ähnlich wie die Filme, ein sehr kleines Plateau dessen Höhe nicht mit der von Polykristallen verglichen werden kann. Aus diesem Faktum und den restlichen Messungen können einige interessante Schlüsse über die hergestellten Proben und das untersuchte Phänomen der Ladungsordnung gezogen werden.

\subsection{Probenqualität}

\subsubsection{Filme auf $\mathrm{MgO}$}

Bei der Probenqualität müssen die auf $\mathrm{MgO}$ hergestellten Filme und die freistehenden Filme getrennt betrachtet werden. Die auf MgO hergestellten Filme zeigen im Röntgendiffraktogramm hauptsächlich eine Orientierung. Es ist bei den meisten Proben unterhalb von $\mathrm{x}=0,75$ ein kleiner (011) Peak sichtbar. Oberhalb von $\mathrm{x}>0,8$ scheint es eine Änderung der Wachstumsrichtung entlang [011] zu geben. Die Proben oberhalb von $\mathrm{x}=0,9$ zeigen typischerweise keinen $<001>$ Peak mehr. Sie wachsen nur noch entlang [011] auf. Die Filme scheinen jedoch entlang der [100] Richtung verzwillingt zu sein. Dies wird besonders dadurch deutlich, dass sich die Ramanspektren der beiden Konfigurationen XX und YY nicht unterscheiden. Die beiden Elemente des Ramantensors $A_{g}$, a und b sind nahezu gleich groß. Der Unterschied ist sogar typischerweise so klein, dass in älteren Veröffentlichungen, die mit Quellen von geringerer Intensität arbeiteten, der Ramantensor mit nur zwei unterschiedlichen Elementen angegeben wurde 61. Die gemessenen Intensitätsverhältnisse sind aber nur möglich, wenn eine Verzwillingung vorliegt, wie im Appendix ausgeführt. Außerdem zeigen auch die TEM-Messungen die Verzwillingung der Proben. Die Größe der Zwillingsdomänen konnte aus den TEM-Messungen zu $30 \mathrm{~nm}$ bestimmt werden.

Bezüglich der regelmäßigen Verteilung von Lanthan und Calcium beziehungsweise möglicher Ausscheidungen lässt sich sagen, dass die Ordnungszahl von Lanthan $Z=57$ beträgt, während Calcium die Ordnungszahl $Z=20$ besitzt. Damit wird deutlich, dass eine kleine Schwankung der Verteilung zu einem großen Kontrast im STEM Bild führt. Ein EELS Spektrum der Probe ist bisher noch nicht durchgeführt worden. Es ist aber aufgrund der hohen Kris- 
tallinität davon auszugehen, dass die Schwankungen in La/Ca zumindest gering sind, da große Schwankungen den Toleranzfaktor und damit die ganze Kristallstruktur nachhaltig negativ beeinflussen würden.

In beiden untersuchten Lamellen ist selbst bei Raumtemperatur eine zusätzliche Periodizität sichtbar. Man erwartet vom Ursprung zum MgO (200) Peak wandernd nur einen weiteren Peak sehr geringer Intensität, den (020) Peak der Pnma Struktur. Dies ist auch bestätigt durch die XRD-Messungen. Im TEM werden jedoch drei weitere Peaks sichtbar. Dies deutet auf eine weitere Periodizität mit der doppelten Gitterkonstante hin. Diese Periodizität könnte zum Beispiel durch eine A-Ordnung entstehen. Die ideal dotierten Manganate mit Vierteldotierung haben diese Eigenschaft bereits gezeigt 73 . Dies würde bedeuten, dass neben einer $L a_{0,5} \mathrm{Ca}_{0,5} \mathrm{MnO}_{3}$ Lage jeweils eine $\mathrm{CaMnO}$ Lage läge. Außerdem möglich ist, dass die Überlagerung der Beugungsbilder der verschiedenen Zwillingsvarianten zu diesen zusätzlichen Peaks führen kann, sodass deren Ursprung weiter untersucht werden muss. Doppelbeugung als Ursache für die zusätzlichen Reflexe ist aufgrund der geringen Dicke der Lamelle eher unwahrscheinlich.

Die Qualität der Proben auf $\mathrm{MgO}$ ist trotz der Defekte sehr viel besser als die oft untersuchten Keramiken. Dies lässt sich durch folgende Argumente belegen:

- Vergleicht man die Ramanspektren, der vermessenen Proben mit den an Polykristallen aufgenommenen Spektren, wird deutlich, dass die Spektren der Polykristalle viel weniger Ramanlinien und deutlich breitere Ramanlinien zeigen. Die Verbreiterung der Ramanlinien und das Fehlen von Linien kann durch starke Unordnung im System erklärt werden [83. Die Spektren von einkristallinem LMO und CMO zeigen eine deutlich stärkere Ähnlichkeit mit den hergestellten Schichten 83,94.

- Des Weiteren können auch die gemessenen THz-Spektren als Qualitätskriterium herangezogen werden. Aufgrund der geringen Wellenzahl des BosonPeaks konnte dieser in den freistehenden Schichten vermessen werden und zeigt eine deutlich geringere Intensität als für die Keramiken siehe Abb. 40. Die Peakposition liegt dabei sehr nahe an der van-Hove-Singularität und impliziert damit eine sehr geringe Unordnung im System [55]. Die bis hierhin mit $\mathrm{THz}$ vermessenen Filme sind nur die zuerst hergestellten Filme. Viele der deutlich hochqualitativeren Schichten, die im Verlauf der Arbeit noch hergestellt wurden, sind in Stuttgart noch nicht vermessen worden.

- Außerdem zeigen die TEM-Messungen ein einkristallines Wachstum und wenige Verunreinigungen oder Grenzflächen, wie sie aus Polykristallen bekannt sind [46. 
- Die richtige Dotierung der hergestellten Proben kann mit hoher Genauigkeit anhand der Auslöschung des (001) Peaks und der Gitterkonstante bestimmt werden.

Auch die Position des Ladungsordnungsübergangs in den Magnetisierungsmessungen stimmt mit der aus anderen Veröffentlichungen überein $38,78,79]$. Es gibt keinen weiteren Übergang der zum Beispiel auf $\mathrm{Mn}_{3} \mathrm{O}_{4}$ zurückgeführt werden könnte.

Die temperaturabhängigen Ramanmessungen haben gezeigt, dass der Symmetriebruch der CO-Phase in den Ramanspektren sichtbar wird. Die Zuordnung der Intensitätsverhältnisse ist ebenfalls nur in der veränderten $P 2_{1} / m$ Struktur möglich. Die Intensitäten der Moden können auch hier wieder wie im Anhang berechnet werden und zeigen, siehe Tabelle 10, dass sich durch die zusätzlichen Diagonalelemente der Matrizen die Intensitäten der einzelnen Konfigurationen stark unterscheiden. Damit ist es gelungen mit Hilfe von temperaturabhängigen Ramanmessungen die Ladungsordnung in den Proben durch den Symmetriebruch von Pnma zu $P 2_{1} / m$ nachzuweisen.

Die Widerstandsdaten legen außerdem nahe, dass die unterschiedlichen Dotierungen sich sehr ähnlich zu den bereits vorhandenen Messungen aus anderen Quellen verhalten [28,31,76].

Die Zusammensetzung der Defekte die zumeist fest umschlossene Gebiete bilden kann außer aus EELS Messungen nicht bestimmt werden. Es kann jedoch vermutet werden, dass es sich anstatt um eine Mangan eher um einen Calcium Defekt handelt, ähnlich den Grenzflächen bei Turner et. al. [95]. Diese Bereiche könnten damit möglicherweise für das kleine ferromagnetische Moment verantwortlich sein, da ihr Volumen sehr gering ist. 


\subsection{2 freistehende Filme}

Die freistehenden Filme zeigen eine sechszählige Symmetrie im Phi-Scan. Sie weisen außerdem zwei Orientierungen auf, (001) und (011). Die Filme sind als verzwillingte und möglicherweise texturierte Polykristalle anzusehen. Es ist möglich, dass sich verschiedene Kristallite entlang der Dreiecksachsen der (111) Oberfläche des MgO bilden. Dies würde ebenfalls zu der $60^{\circ}$-Symmetrie im Phi-Scan führen.

Die freistehenden Filme zeigen ein FIR-Spektrum, das dem der Keramiken ähnlich ist. Die Linien sind scharf und wohl definiert.

Die THz-Spektren der Filme geben weiterhin Aufschluss darüber, dass der Boson-Peak, der ein Maß für die Unordnung des Systems ist, in den freistehenden Filmen eine kleinere Intensität und eine Verschiebung zu höheren Wellenzahlen zeigt, im Vergleich zu den Keramiken. Dies zeigt eine schwächer ausgeprägte Wechselwirkung der ungeordneten Bereiche oder eine geringere Anzahl an ungeordneten Bereichen [55].

Die freistehenden Filme zeigen somit eine bessere Ordnung als die Keramiken [81].

Da für die freistehenden Filme das gleiche Rezept verwendet wurde wie für die Herstellung der Filme auf $\mathrm{MgO}$, ist davon auszugehen, dass auch hier die Dotierungen sehr genau mit den beabsichtigten übereinstimmen. 


\section{$5.2 \quad$ Ladungsordnung}

Auch bei der Ladungsordnung müssen die beiden Probenarten unterschieden werden.

Die auf $\mathrm{MgO}$ hergestellten Filme zeigen Ladungsordnungscharakteristika. So weisen sie, in der Magnetisierung, einen Peak bei der Ladungsordnungstemperatur auf.

Die temperaturabhängigen Ramanmessungen zeigen den Symmetriebruch durch die Ladungsordnung.

Einige Proben zeigen ein kleines Plateau im Widerstand, unterhalb der Ladungsordnungstemperatur. Alle Proben deren Dotierung im ladungsgeordnetem Bereich liegt zeigen unterhalb der Ladungsordnungstemperatur einen starken Anstieg des Widerstands mit sinkender Temperatur.

Die Auftragung von $\frac{d \ln (\rho)}{d T^{-1}}$ gegen die Temperatur zeigt ebenfalls den Ladungsordnungsübergang. Die geringeren Werte im Vergleich zu Polykristallen könnten aber darauf hindeuten, dass die Ladungsordnung in den Filmen anders ausgeprägt ist. Des Weiteren ist zu beachten, dass eine Widerstandsmessung im strengsten Sinne keine globale Messung ist. Das bedeutet der Strom sucht sich den Pfad des geringsten Widerstands in der Probe. Wenn die Filme Defekte oder Zwillingsgrenzen aufweisen ist es also möglich, dass das Plateau im Widerstand durch Pfade entlang von defektreichen Gebieten oder Zwillingsgrenzen ausgeschmiert wird oder gar nicht erst sichtbar wird.

Die TEM-Messungen zeigen ebenfalls für beide Lamellen Ladungsordnung. Diese ist jedoch anders ausgeprägt als nach dem Wigner-Crystal Modell erwartet würde.

Aufgrund der Phononenbanden des $\mathrm{MgO}$ konnte an den Filmen auf $\mathrm{MgO}$ keine FIR-Spektroskopie durchgeführt werden. Damit ist es nicht möglich die Überstrukturpeaks im FIR-Spektrum zu messen. Allerdings ist aufgrund der Verzwillingung zu erwarten, dass die Größe der ladungsgeordneten Domänen begrenzt ist durch die Größe der Zwillinge [50]. Damit deutet vieles daraufhin, dass die Ladungsordnung in den Schichten auf $\mathrm{MgO}$ vorhanden aber schwächer als in Polykristallen ist.

Bei den freistehenden Filmen wurden Magnetisierungsmessungen durchgeführt bevor das Substrat entfernt wurde. Die vermessenen Proben zeigen ein kleines ferromagnetisches Moment, was aufgrund der vielen Grenzflächen zu erwarten war. Das Moment ist jedoch groß genug um den Ladungsordnungsübergang zu überdecken. Er ist in den freistehenden Filmen in der Magnetisierung nicht sichtbar.

Die Leitfähigkeit die über die THz-Messungen ermittelt wurde, zeigt ebenfalls nur einen sehr kleinen Sprung bei der Ordnungstemperatur. Die Temperaturauflösung war bei den THz Messungen sehr gering, was hauptsächlich der 
geringen Lebenszeit der Quellen geschuldet ist. Damit kann auch hier keine eindeutige Aussage über die Ladungsordnung getroffen werden.

Die FIR-Messungen die eines der besten Indizien stellen über die Peaks in der Leitfähigkeit, die durch die Faltung der Brillouin-Zone entstehen, können ebenfalls nicht verwendet werden, da der Fit für den unterliegenden Tesafilmstreifen, im Bereich der Überstrukturpeaks, extrem weit von den wirklichen Werten deviiert. Damit kann auch hier keine Aussage getroffen werden, ob die freistehenden Filme Ladungsordnung zeigen oder nicht. Das erwartete Bild der Ladungsordnung mit der kommensurablen Überstruktur von $a^{*}=4 a_{0}$ kann in den TEM Messungen nicht verifiziert werden. Die aus dem Beugungsbild der parallel zum Substrat geschnittenen Lamelle bestimmten Gitterkonstante zeigt eine extrem starke Änderung mit der Temperatur und sollte daher zu einer Änderung der Kristallstruktur führen. Die genaue Struktur der Ladungsordnung in den $\mathrm{x}=0,75$ dotierten Proben bleibt unklar. Es gibt eine zusätzliche Periodizität in beiden Lamellen, die durch eine APlatz Ordnung erklärt werden kann. Ein strukturelles Modell kann jedoch erst nach EELS Messungen erstellt werden. Die drastische Änderung der Gitterkonstante in der parallelen Lamelle kann mit den zur Zeit vorliegenden Modellen nicht erklärt werden. Es muss außerdem festgestellt werden, inwiefern die Defekte die Eigenschaften der Probe beeinflussen oder eine intrinsische Phasenseperation darstellen. 


\subsection{Zusammenhang der verschiedenen Ordnungs- und Unordnungsskalen}

In den überdotierten LCMO Proben müssen drei Ordnungsparameter zueinander in Relation gesetzt werden.

1. Die Kristallinität der Probe und damit die langreichweitige Ordnung der Manganatphase.

2. Die Ladungsordnung der Probe, die einhergeht mit einer orbitalen Ordnung und der Überstruktur.

3. Die Nahordnung der Probe, die hauptsächlich bestimmt wird durch die Größe der Zwillinge und anderer Störstellen.

Je besser die Kristallinität der Probe, desto schärfer sind Raman-, Röntgen und Infrarotpeaks. Die Ladungsordnung in der Probe ist insofern mit der Kristallinität verknüpft, als das die Ladungsordnung eine gewisse minimale Domänengröße benötigt [96, 97]. Wird diese minimale Domänengröße unterschritten, so ist ein ferromagnetisches Moment in der Magnetisierung zu sehen 96, 98. Wenn die Kristallinität der Probe nicht ausreichend ist, kann keine Ladungsordnung existieren.

Die Randbereiche der ladungsgeordneten Phase sind dabei das Gegenteil von den AFM-Fluktuationen, die in den CMR-Manganaten auftreten. Die Kopplung der Phasengrenzen ist ferromagnetisch und durch Reduktion der Domänengröße kann man, von einem isolierendem ladungsgeordnetem Zustand, in einen ferromagnetisch metallischen Zustand gelangen [96, 98, 99]. Für die Größe der ladungsgeordneten Domänen in den vorliegenden Proben kann damit zumindest eine untere Grenze festgelegt werden. So müssen die Domänen mindestens eine Größe von 30nm aufweisen [96-98].

Die Nahordnung der Probe wird hauptsächlich durch den Bosonpeak charakterisiert, da hier die kurzreichweitigen Wechselwirkungen der Störstellen zu berücksichtigen sind. Die Nahordnung bedingt natürlich ebenfalls die Breite der Röntgenpeaks. Sie gibt außerdem Aufschluss über die Verzwillingung der ladungsgeordneten Phase.

Damit ergibt sich ein komplexes Wechselspiel der einzelnen Ordnungsparameter. Dabei ist die Ladungsordnung die Ordnung, die am einfachsten beeinflusst werden kann. Kleine Dotierungsunterschiede, eine zu geringe Sauerstoffkonzentration oder Störstellen können schon bei sehr kleinen Konzentrationen die Ladungsordnung komplett unterdrücken [33, 96, 98. Die Tatsache, dass sie dennoch in mehreren Messungen sichtbar gemacht werden kann, bedeutet, dass ein messbarer Teil der Proben Ladungsordnung zeigt.

Mit der MAD-Technik ist es möglich überdotiertes LCMO mit Schichtdicken von über $500 \mathrm{~nm}$ herzustellen. Die Proben zeigen die charakteristischen Eigenschaften, die auch schon in Polykristallen beobachtet wurden, sind dabei 
aber einkristallin entlang der c-Achse aufgewachsen.

Die hergestellten Proben stellen den Kontrapunkt zu den viel untersuchten $\mathrm{x}=0,33$ LCMO Proben dar. Anstatt eines Isolatormetallübergangs zeigen die Proben, unterhalb der Ordnungstemperatur, einen Metallisolatorübergang. Die entstehende Phase zeigt ein magnetisches Moment, das durch den Doppelaustausch in Kombination mit dem Superaustausch und der Struktur der ladungsgeordneten Phase mediiert wird [26]. Die Phase kann ebenfalls durch sehr hohe Magnetfelder zerstört werden und führt zu einem metallisch ferromagnetischem Zustand, genau wie in den CMR-Manganaten [27, 100]. Die Übergangstemperaturen liegen hierbei in ähnlichen Bereichen wie bei den CMR-Manganaten.

Die Ladungsordnungsstruktur die in den Filmen beobachtet wird ist unterschiedlich zum Wigner-Crystal Modell. In den beiden Abbildungen 7 und 8 ist eine Verzwillingung entlang der [101]-Richtung berücksichtigt worden, wie sie in den vorliegenden Proben vorliegt. Dabei sind von den grün eingezeichneten, frustrierten Bindungen in der Wignerstruktur 6 pro Einheitszelle vorhanden, während im Bi-Stripe-Modell ebenfalls 6 frustrierte Bindungen pro Einheitszelle zu verzeichnen sind. Ist die Probe verzwillingt erhöht sich jedoch die Anzahl der frustrierten Bindungen im Bi-Stripe-Modell auf 8, während für die Wigner-Crystal-Struktur die Anzahl konstant bleibt. Dies bedeutet, dass die Wigner-Crystal-Struktur bei einer verzwillingten Probe bevorzugt werden sollte, da jede dieser frustrierten Bindungen die Energie des Gitters erhöht. Gleichzeitig müssen aber auch andere Energieskalen, wie die Gitterenergie und Doppelaustauschenergie, berücksichtigt werden, wodurch hier nur eine tendenzielle Aussage getroffen werden kann. Sie ist bei tiefen Temperaturen schwächer ausgeprägt. Dies könnte auch der Grund sein, dass im spezifischen Widerstand kein starker Sprung beobachtet werden kann. Ob die Verunreinigungen, die in der parallel geschnittenen Lamelle sichtbar wurden, einen Einfluss auf die Struktur haben kann zu diesem Zeitpunkt nicht festgestellt werden. Das magnetische Moment, das beim Ladungsordnungsübergang beobachtet wird, ist jedoch kein Effekt der Verunreinigungen. Zu sehr korreliert das Moment mit der Ladungsordnungstemperatur. Außerdem lässt die geringe Größe des Moments weitere ferro- oder ferrimagnetische Verunreinigungen wie $\mathrm{Mn}_{3} \mathrm{O}_{4}$ unwahrscheinlich erscheinen. Eine Zuordnung alleine über den TERS-Peak ist nicht möglich.

Die $\mathrm{M}(\mathrm{H})$ Kurven zeigen für die hochdotierten Proben ein remanentes magnetisches Moment, das eine Phasenseparation wahrscheinlich erscheinen lässt. Die Änderung der Ramanmodenposition mit der Temperatur in der $\mathrm{x}=0,96$ dotierten Probe lässt jedoch eher einen Bulk-Effekt vermuten. Die Abweichung vom anharmonischen Modell kann möglicherweise mit ferromagnetischen Momenten in einer antiferromagnetischen Matrix erklärt werden. Hier- 
bei entsteht der Modenshift durch die antiferromagnetische Kopplung und es sollte bei extrem tiefen Temperaturen eine Aufspaltung der Moden beobachtet werden können.

Der Symmetriebruch in den Ramanmessungen, der Probe mit Dotierung $\mathrm{x}=0,75$, zeigt wie bereits in der Theorie vorhergesagt den Phasenübergang von der Pnma Phase zur $P 2_{1} / m$ Phase. Die Pnma Struktur ist nur stabil bis zu einer Verdreifachung einer der Gitterkonstanten. Für die kommensurable Überstruktur von $\mathrm{x}=0,75$ ist jedoch eine Vervierfachung der Gitterkonstante der a-Achse notwendig. Hieraus ergibt sich die Notwendigkeit eines strukturellen Phasenübergangs. Dieser Phasenübergang wurde bereits in den Messungen von Pissas beobachtet [33|. Dieser Phasenübergang lässt sich auch in den Ramanmessungen eindeutig finden:

Durch zusätzlich auftretende Moden und wie bisher bereits diskutiert die Übereinstimmung von theoretisch berechneten Intensitätsverhältnissen und experimentell ermittelten.

Ein weiteres interessantes Gebiet liegt im Bereich von $\mathrm{x}=0,96$. Das dauerhafte Streitthema ist hier, ob eine ferromagnetische Phase in einer antiferromagnetischen Matrix vorliegt, oder das Material ein gekanteter Antiferromagnet ist. Anhand der vorliegenden Experimente lässt sich Folgendes sagen. Das Material zeigt in Magnetisierungsmessungen eine große Hysterese und zwei unterschiedliche Remanenzen in $\mathrm{M}(\mathrm{H})$. Das magnetische Moment aus $\mathrm{M}(\mathrm{H})$ im Vergleich zu einem durch die Dzyaloshinskii-Moriya-Wechselwirkung verkanteten Moment ist zu groß. Das Moment aus der Dzyaloshinskii-MoriyaWechselwirkung ist proportional zur Spinbahnwechselwirkung die wiederum proportional ist zur Ordnungszahl Z. Das kleine Z von Mangan, sowie die geringe Verkippung der Oktaeder $[28 \mid$ sorgt dafür, dass die DzyaloshinskiiMoriya-Wechselwirkung ein deutlich kleineres Moment zeigen würde als das hier vorliegende.

Die Ramanmessungen zeigen zweierlei Effekte. Zum einen lässt sich sagen, dass unterhalb von $\mathrm{T}=150 \mathrm{~K}$ die Intensitätsverhältnisse der einzelnen Moden nicht mehr zu den vorher berechneten Verhältnissen passen. Hier möglicherweise bedingt durch eine ferromagnetische Phase die gleichzeitig mit der AFM-Phase auftritt und deren Modenpositionen sehr nahe an denen der AFM-Phase liegen. Zum anderen lässt sich zeigen, dass ein Fit des anharmonischen Modells der Modenposition von $\mathrm{x}=0,96$ eine gute Übereinstimmung liefert bis $\mathrm{T}=150 \mathrm{~K}$. Die Modenposition von $\mathrm{T}=50 \mathrm{~K}$ weicht deutlich vom anharmonischen Modell ab. Dies lässt eher darauf schließen, dass eine globale Phase vorhanden ist. Außerdem möglich wäre auch eine Phasenseparation bei der die Modenposition nahe der AFM-Phase liegt und die daher eine Verbreiterung des Peaks verursacht. Die dafür nötige Verbreiterung der Peaks kann nicht beobachtet werden. 


\section{Ausblick}

Die in der Arbeit hergestellten LCMO Proben sind die ersten Manganatproben die mit dem neuen TERS Aufbau vermessen werden konnten. Die ersten Messungen zeigen, dass abhängig von der Position der Spitze ein anderes Ramanspektrum aufgenommen werden kann. Die LCMO und LSMO Proben, die den CMR-Effekt zeigen, haben typischerweise ein sehr kleines Ramansignal, das mit der seitlichen Illumination des TERS Aufbaus nicht gemessen werden kann. Wenn gar kein Signal vorhanden ist, ist es darum umso schwieriger eine TERS Verstärkung zu erreichen, da die Kalibration nur abhängig von einem Referenzsignal ausgeführt werden kann. Die Proben können, da sie Bereiche zeigen die den korrelierten Polaronen sehr verwandt sind, weiter untersucht werden, um zu klären ob die Auflösung des TERS genügt um diese Bereiche und ihre strukturellen Eigenschaften sichtbar zu machen. Hierbei sollte abhängig von der Position der Spitze ein Ramansignal mit einem deutlich verschiedenen Modenspektrum gemessen werden. Die Messungen die bisher durchgeführt wurden, zeigen hauptsächlich einen zusätzlichen Peak der von der Position der Spitze auf der Probe abhängig ist. Die zusätzlich auftauchende Linie kann dabei viele mögliche Auslöser haben, die noch nicht schlussendlich geklärt sind. Eine Strukturänderung in hochdotiertem LCMO bei Laserillumination wurde bereits berichtet [8], sodass ähnliche Proben möglicherweise für den SFB 1073 verwendet werden können. Fabio Boschini vom Dipartimento di Fisica de Politecnico de Milano hat bei energieaufgelösten Messungen der Reflektivität, an den in dieser Arbeit hergestellten Proben, eine ähnliche Relaxation mit viel größerer Lebensdauer beobachtet.

Die Ramanmessungen die an den unterschiedlichen Proben aufgenommen wurden, sollten unbedingt mit weiteren Messungen bei tieferen Temperaturen der anderen Dotierungen ergänzt werden. Dabei ist bei den Dotierungen $0,5 \leq x \leq 0,85$ ein Symmetriebruch und somit ein deutlich anderes Ramansignal zu erwarten. Hierbei ist immer darauf zu achten, dass die Proben Verzwillingung zeigen können und die Intensitätsverhältnisse sehr stark von denen eines Einkristalls abweichen.

Des Weiteren sollten auch TEM Messungen an freistehenden Filmen durchgeführt werden, um zum einen die Struktur und zum anderen das Wachstum der Bufferschichten zu untersuchen. Außerdem ist es mit EELS möglich die Ladungsordnung aufzulösen, da bis jetzt nicht abschließend geklärt werden konnte, ob diese in den freistehenden Schichten vorhanden ist.

Für die weitere Untersuchung, ob sich außerdem eine Ladungsdichtewelle finden lässt, wäre es ebenfalls wichtig den Frequenzraum noch um den GHZBereich zu erweitern, in dem typischerweise Ladungsdichtewellen eine Reso- 
nanz zeigen. Es wäre außerdem das erste vollständige Frequenzspektrum der ladungsgeordneten LCMO Proben.

Es sollten außerdem Messungen mit einem Vierkreisgoniometer bei tiefen Temperaturen durchgeführt werden, um die TEM-Messungen zu bestätigen und bei der weiteren Herstellung der Proben eine Möglichkeit zu finden, durch Änderung der Stöchiometrie oder des Substrats, eine Verzwillingung zu vermeiden. Dadurch sollte auch eine stärkere Ladungsordnung erreicht werden können.

Die hier vorgestellte Art um freistehende Schichten zu produzieren kann auch für andere Oxide verwendet werden, solange diese basisch sind und von der Natronlauge nicht angegriffen werden. Es wäre aber nötig eine Möglichkeit zu finden, um von der angenommenen hexagonalen Struktur des $\mathrm{ZnO}$, wieder zu einer kubischen Struktur überzugehen. Hierfür sind möglicherweise weitere Bufferlayer und weiterführende Versuche nötig. Es ist aber eine vielversprechende Technik, da die freistehenden Filme trotz der Behandlung und der Tatsache, dass sie sehr porös sind ein sehr gutes FIR Signal geliefert haben, was als gutes Indiz für eine intakte Struktur gesehen werden kann. Mithilfe dieser Technik ist es auch möglich die sonst nötigen invasiven Schritte bei der Probenpräperation für spektroskopische Messungen zur eigentlichen Herstellung auszulagern und somit größere Autonomie zu erreichen.

Mit der neuen lagenweisen Deposition, die die MAD3 ermöglicht, sollten außerdem Übergitter hergestellt werden, die aus unterschiedlich dotierten Lagen bestehen. Damit sollte es möglich sein zu bestimmen, ob die Streifenstrukturen, die in vielen TEM Arbeiten beobachtet werden, ein Phänomen der Unordnung der Polykristalle sind, oder sich wirklich eine Phasensepara-

tion in die kommensurablen Phasen fortsetzt. Dies gäbe auch Aufschluss auf die Größenordnung der Antiphasengrenzen, die in den CMR-Manganaten so wichtig sind. Dadurch könnten artifiziell hergestellte CMR-Schichten erzeugt werden, deren Ladungsordnung stark ist, aber sehr empfindlich auf Magnetfelder reagiert.

Ob und inwiefern für die Proben mit $\mathrm{x}=0,75$ ein Übergang von einer Ladungsordnung zu einer anderen Ladungsordnung erfolgt, muss weiter untersucht werden. Die Einflüsse der möglichen A-Platz Ordnung sollten dabei unbedingt beachtet werden. 


\section{Literatur}

\section{Literatur}

[1] R. von Helmolt, J. Wecker, B. Holzapfel, L. Schultz, and K. Samwer. Giant negative magnetoresistance in perovskitelike $L a_{2 / 3} \mathrm{Ba}_{1 / 3} M n \mathrm{O}_{x}$ ferromagnetic films. Physical Review Letters, 71(14):2331-2333, 1993.

[2] Y. Tokura and Y. Tomioka. Colossal magnetoresistive manganites. Journal of Magnetism and Magnetic Materials, 200(1-3):1-23, October 1999.

[3] G. Q. Gong, C. Canedy, G. Xiao, J. Z. Sun, A. Gupta, and W. J. Gallagher. Colossal magnetoresistance of 1000000-fold magnitude achieved in the antiferromagnetic phase of $L a_{1-x} C a_{x} M n O 3$. Applied Physics Letters, 67(1995):1783, 1995.

[4] G. C. Xiong, Q. Li, H. L. Ju, S. N. Mao, L. Senapati, X. X. Xi, R. L. Greene, and T. Venkatesan. Giant magnetoresistance in epitaxial $\mathrm{Nd}_{0.7} \mathrm{Sr}_{0.3} \mathrm{MnO}_{3-\delta}$ thin films. Applied Physics Letters, 66(1995):1427, 1995.

[5] E. Dagotto. Open questions in CMR manganites, relevance of clustered states and analogies with other compounds including the cuprates. New Journal of Physics, 7:67-67, February 2005.

[6] M. Uehara, S. Mori, C.H. Chen, and S.-W. Cheong. Percolative phase separation underlies colossal magnetoresistance in mixed-valent manganites. Nature, 399(June), 1999.

[7] B. Raveau, M. Hervieu, A. Maignan, and C. Martin. The route to CMR manganites: what about charge ordering and phase separation? Journal of Materials Chemistry, 11(1):29-36, 2001.

[8] P. Beaud, S. Johnson, E. Vorobeva, U. Staub, R. Souza, C. Milne, Q. Jia, and G. Ingold. Ultrafast Structural Phase Transition Driven by Photoinduced Melting of Charge and Orbital Order. Physical Review Letters, 103(15):155702, October 2009.

[9] J. B. Goodenough. Theory of the role of covalence in the perovskitetype manganites $[\mathrm{La}, \mathrm{M}(\mathrm{II})] \mathrm{MnO}_{3}$. Physical Review, 100(1951):564$573,1955$. 
[10] T. Zhang, E. Zhukova, B. Gorshunov, D. Wu, A. S. Prokhorov, V. I. Torgashev, E. G. Maksimov, and M. Dressel. Terahertz spectroscopy of low-energy excitations in charge-ordered $L a_{0.25} \mathrm{Ca}_{0.75} \mathrm{MnO}_{3}$. $\underline{\text { Physical }}$ Review B, 81(12):125132, March 2010.

[11] V. Moshnyaga, A. Giske, and K. Samwer. Giant negative photoconductivity in $\mathrm{La}_{0.7} \mathrm{Ca}_{0.3} \mathrm{MnO}_{3}$ thin films. Journal of Applied Physics, 95(11):7360, 2004.

[12] J. Tao, D. Niebieskikwiat, M. Varela, W. Luo, M. A. Schofield, Y. Zhu, M. B. Salamon, J. M. Zuo, S. T. Pantelides, and S. J. Pennycook. Direct imaging of nanoscale phase separation in $L a_{0.55} \mathrm{Ca}_{0.45} \mathrm{MnO}_{3}$ : Relationship to colossal magnetoresistance. Physical Review Letters, 103(August):1-4, 2009.

[13] H. T. Yi, T. Choi, and S.-W. Cheong. Reversible colossal resistance switching in $(\mathrm{La}, \mathrm{Pr}, \mathrm{Ca}) \mathrm{MnO}_{3}$ : Cryogenic nonvolatile memories. Applied Physics Letters, 95(2009):063509, 2009.

[14] J. O. Krisponeit, C. Kalkert, B. Damaschke, V. Moshnyaga, and K. Samwer. Nanoscale resistance switching in manganite thin films: Sharp voltage threshold and pulse-width dependence. Physical Review B - Condensed Matter and Materials Physics, 82:1-6, 2010.

[15] M. B. Salamon and M. Jaime. The physics of manganites: Structure and transport. Reviews of Modern Physics, 73(July):583-628, 2001.

[16] J. P. Attfield. A Simple Approach to Lattice Effects in Conducting Perovskite-Type Oxides. Chemistry of Materials, 10:3239-3248, 1998.

[17] H. A. Jahn and E. Teller. Stability of polyatomic molecules in degenerate electronic states. i. orbital degeneracy. Proceedings of the Royal Society of London. Series A, Mathematical and Physical Sciences, pages 220-235, 1937.

[18] A. J. Millis. Cooperative Jahn-Teller effect and electron-phonon coupling in $\mathrm{La}_{1-x} \mathrm{Ca}_{x} \mathrm{MnO}_{3}$. Physical Review B, 53(13):8434-8441, 1996.

[19] C. Zener. Interaction between the d-shells in the transition metals. II. Ferromagnetic compounds of manganese with perovskite structure. Physical Review, 82(3):403-405, 1951.

[20] P. W. Anderson and H. Hasegawa. Considerations on Double Exchange. Physical Review, 100(2), October 1955. 
[21] M. J. Caldero and L. Brey. Monte Carlo simulations for the magnetic phase diagram of the double-exchange Hamiltonian. Physical Review B, 58(6):3286, August 1998.

[22] M. B. Salamon, P. Lin, and S. H. Chun. Colossal magnetoresistance is a Griffiths singularity. Physical review letters, 88:197203, 2002.

[23] P. W. Anderson. Antiferromagnetism. Theory of superexchange interaction. Physical Review, 79(1934), 1950.

[24] L. F. Tocchio, F. Becca, A. Parola, and S. Sorella. Role of backflow correlations for the nonmagnetic phase of the $t-t$ Hubbard model. Physical Review B - Condensed Matter and Materials Physics, 78:1-4, 2008.

[25] A. Flesch, G. Zhang, E. Koch, and E. Pavarini. Orbital-order melting in rare-earth manganites: Role of superexchange. Physical Review B Condensed Matter and Materials Physics, 85:1-5, 2012.

[26] H. Aliaga, D. Magnoux, A. Moreo, D. Poilblanc, S. Yunoki, and E. Dagotto. Theoretical study of half-doped models for manganites: Fragility of CE phase with disorder, two types of colossal magnetoresistance, and charge-ordered states for electron-doped materials. Physical Review B, 68(10):104405, September 2003.

[27] C. N. R. Rao and A. Arulraj. Charge ordering in the rare earth manganates: the experimental situation. Journal of Physics: Condensed Matter, pages 83-106, 2000.

[28] M. Pissas and G. Kallias. Phase diagram of the $\mathrm{La}_{1-x} \mathrm{Ca}_{x} \mathrm{MnO}_{3}$ compound $(0.5 \leq x \leq 0.9)$. Physical Review B, 68(13):134414, October 2003 .

[29] D Shulyatev, S Karabashev, A Arsenov, and Ya Mukovskii. Growth and investigation of doped rare earth manganite single crystals. Journal of Crystal Growth, 198-199:511-515, March 1999.

[30] A. M. Balbashov, S.G. Karabashev, Y. M. Mukovskiy, and S.A. Zverkov. Growth and giant magnetoresistance effect in $L a_{1-x} \mathrm{Ca}_{x} \mathrm{MnO}_{3}$ and $\mathrm{La}_{1-x} \mathrm{Sr}_{x} \mathrm{MnO}_{3}$ single crystals. Journal of Crystal Growth, 167 (1-2):365-368, September 1996.

[31] Y. Wang, Yu Sui, H. Fan, X. Wang, Y. Su, W. Su, and X. Liu. High Temperature Thermoelectric Response of Electron-Doped $\mathrm{CaMnO}_{3}$. Chemistry of Materials, 21(19):4653-4660, October 2009. 
[32] N. N. Loshkareva, A. V. Korolev, N. I. Solin, E. V. Mostovshchikova, S. V. Naumov, and A. M. Balbashov. Magnetic, electrical, and optical properties of electron-doped $\mathrm{Ca}_{1-x} \mathrm{La}_{x} \mathrm{MnO}_{3-\delta}(x \leq 0.12)$ single crystals. Journal of Experimental and Theoretical Physics, 102(2): 248-257, February 2006.

[33] M. Pissas, I. Margiolaki, K. Prassides, and E. Suard. Crystal and magnetic structural study of the $\mathrm{La}_{1-x} \mathrm{Ca}_{x} \mathrm{MnO}_{3}$ compound $(x=3 / 4)$. Physical Review B, 72(6):064426, August 2005.

[34] M. Calandra, J. Merino, and Ross H. McKenzie. Metal-insulator transition and charge ordering in the extended Hubbard model at one-quarter filling. Physical Review B, (66):195102, November 2002.

[35] P. G. Radaelli, D. E. Cox, and L. Capogna. Wigner-crystal and bistripe models for the magnetic and crystallographic superstructures of $\mathrm{La}_{0.333} \mathrm{Ca}_{0.667} \mathrm{MnO}_{3}$. Physical Review B, 59(22):440-450, 1999.

[36] S. Mori, T. Asaka, and Y. Matsui. Observation of magnetic domain structure in phase-separated manganites by Lorentz electron microscopy. Journal of electron microscopy, 51(4):225-229, 2002.

[37] M. Coey. Charge ordering in oxides. Nature, 430(July):155-157, 2004.

[38] X. H. Huang, Z. L. Jiang, X. F. Sun, and X. G. Li. Exchange Bias Effect Induced by the Intrinsic Inhomogeneity in $\mathrm{La}_{1-x} \mathrm{Ca}_{x} \mathrm{MnO}_{3}(0.55 \leq$ $x \leq 0.95)$ Compounds. Journal of the American Ceramic Society, 94 (5):1324-1326, May 2011.

[39] C. Lu, N. Hu, M. Yang, S. Xia, H. Wang, J. Wang, Z. Xia, and J.-M. Liu. High magnetic field phase diagram in electron-doped manganites $L a_{0.4} \mathrm{Ca}_{0.6} \mathrm{Mn}_{1-y} \mathrm{Cr}_{y} \mathrm{O}_{3}$. Scientific reports, 4:4902, January 2014.

[40] P. Kumar, R. Prasad, A.K. Srivastava, N. Vijayan, R.K. Dwivedi, and H.K. Singh. Microstructural and magnetotransport properties of $\mathrm{La}_{1-x} \mathrm{Ca}_{x} \mathrm{MnO}_{3}(0.45 \leq x \leq 0.6)$ thin films. Journal of Alloys and Compounds, 531:23-29, August 2012.

[41] C. Jooss, L. Wu, T. Beetz, R. F. Klie, M. Beleggia, M. A. Schofield, S. Schramm, J. Hoffmann, and Y. Zhu. Polaron melting and ordering as key mechanisms for colossal resistance effects in manganites. Proceedings of the National Academy of Sciences of the United States of America, 104(34):13597-13602, 2007. 
[42] S. Mori, C. H. Chen, and S.-W. Cheong. Paired and unpaired charge stripes in the ferromagnetic phase of $\mathrm{La}_{0,5} \mathrm{Ca}_{0,5} \mathrm{MnO}_{3}$. Physical review letters, 1(c):1-4, 1998.

[43] N. N. Loshkareva, E. V. Mostovshchikova, N. I. Solin, S. V. Naumov, N. V. Kostromitina, and A. M. Burkhanov. Crossover from polaron to band conduction upon doping $\mathrm{CaMnO}_{3}$ with La ions. JETP Letters, 86(6):375-378, November 2007.

[44] F. L. Tang and X. Zhang. Layer-stripes in charge-ordered $L a_{1-x} \mathrm{Ca}_{x} \mathrm{MnO}_{3} \quad(\mathrm{x}=1 / 3,1 / 2$, and 2/3). Journal of Physics: Condensed Matter, 19(10):106216, March 2007.

[45] S. Mori, C.H. Chen, and S.-W. Cheong. Pairing of charge-ordered stripes in $(\mathrm{La}, \mathrm{Ca}) \mathrm{MnO}_{3}$. Nature, 392:473-476, 1998.

[46] R. Wang, J. Gui, Y. Zhu, and A. Moodenbaugh. Crystallographic analysis of orientational domain variants and charge-ordered domains in $\mathrm{La}_{0,33} \mathrm{Ca}_{0,67} \mathrm{MnO}_{3}$. Physical Review B, 63(14):144106, March 2001.

[47] J. Tao. Unveiling the microscopic origin of the electronic smecticnematic phase transition in $\mathrm{La}_{1 / 3} \mathrm{Ca}_{2 / 3} \mathrm{MnO}_{3}$. arXiv:1403.7216v2, 2014.

[48] Eugene Wigner. On the interaction of electrons in metals. Physical Review, 46(11):1002, 1934.

[49] T. S. Orlova, J. Y. Laval, P. Monod, J. G. Noudem, V. S. Zahvalinskii, V. S. Vikhnin, and Yu P. Stepanov. Effect of fe doping on structure, charge ordering, magnetic and transport properties of $\left.l a_{0.33} \mathrm{ca}_{0.67} \mathrm{mn}_{1-y} f e_{y} \mathrm{O}_{3}(0 \leq y \leq 006)\right)$. Journal of Physics: Condensed Matter, 18:6729-6748, 2006.

[50] J. Tao and J. M. Zuo. Nanoscale phase competition during charge ordering in intrinsically strained $\mathrm{La}_{0.33} \mathrm{Ca}_{0.67} \mathrm{MnO}_{3}$. Physical Review B - Condensed Matter and Materials Physics, 69:67-70, 2004.

[51] P. N. Santhosh, A. Arulraj, P. V. Vanitha, R. S. Singh, K. Sooryanarayana, and C. N. R. Rao. Charge ordering in electron-doped manganates. Journal of Physics: Condensed Matter, 11, 1999.

[52] H. Zhou, G. Li, S. Feng, Y. Liu, T. Qian, X. Fan, and X. Li. The effect of phase separation on charge ordering state in $L a_{1-x} \mathrm{Ca}_{x} \mathrm{MnO}_{3}$ (x = 1/2, 2/3, and 3/4). Solid State Communications, 122:507-510, 2002. 
[53] V. Ponnambalam, S. Parashar, A. R. Raju, and C. N. R. Rao. Electricfield-induced insulator-metal transitions in thin films of charge-ordered rare-earth manganates. Applied Physics Letters, 74(2):206, 1999.

[54] L. van Hove. The occurrence of singularities in the elastic frequency distribution of a crystal. Physical Review, 89(6):1189-1193, 1953.

[55] V. Gurevich, D. Parshin, and H. Schober. Anharmonicity, vibrational instability, and the Boson peak in glasses. Physical Review B, 67(9): 094203, March 2003.

[56] R. Zorn. The boson peak demystified? Physics, 4, 2011.

[57] A. I. Chumakov, G. Monaco, A. Monaco, W. A. Crichton, A. Bosak, R. Rüffer, A. Meyer, F. Kargl, L. Comez, D. Fioretto, H. Giefers, S. Roitsch, G. Wortmann, M. H. Manghnani, A. Hushur, Q. Williams, J. Balogh, K. Parlinski, P. Jochym, and P. Piekarz. Equivalence of the boson peak in glasses to the transverse acoustic van, hove singularity in crystals. Physical Review Letters, 106(June):1-5, 2011.

[58] M. Cardona and Y. Y. Peter. Fundamentals of semiconductors. Springer, 2005.

[59] L. J. van der Pauw. A method of measuring the resistivity and Hall coefficient on lamellae of arbitrary shape. Philips technical review, 1958 .

[60] H. Kim, K. M. Kosuda, R. P. Van Duyne, and P. C. Stair. Resonance Raman and surface- and tip-enhanced Raman spectroscopy methods to study solid catalysts and heterogeneous catalytic reactions. Chemical Society reviews, 39(12):4820-44, December 2010.

[61] R. Loudon. The Raman effect in crystals, volume 13. October 1964. ISBN 0001873640010. 423-482 pp.

[62] I. R. Beattie and T. R. Gilson. Single Crystal Laser Raman Spectroscopy. Proceedings of the Royal Society A: Mathematical, Physical and Engineering Sciences, 307(1491):407-429, November 1968.

[63] M. Abrashev, J. Bäckström, L. Börjesson, M. Pissas, N. Kolev, and M. Iliev. Raman spectroscopy of the charge- and orbital-ordered state in $\mathrm{La}_{0.5} \mathrm{Ca}_{0.5} \mathrm{MnO}_{3}$. Physical Review B, 64(14):144429, September 2001. 
[64] J. R. Jasperse, A. Kahan, and J. N. Plendl. Temperature Dependence of Infrared Dispersion in Ionic Crystals $\mathrm{LiF}$ and $\mathrm{MgO}^{*}$. Physical Review, 146(2):526-542, 1966 .

[65] Z M Zhang, B I Choi, and M I Flik. Infrared refractive indices of $\mathrm{LaAlO}_{3}, \mathrm{LaGaO}_{3}$, and $\mathrm{NdGaO}_{3}$. Journal of Optical Society of America B, 11(11):2252-2257, 1994.

[66] J. Humlíček, R. Henn, and M. Cardona. Infrared vibrations in $\mathrm{LaSrGaO}_{4}$ and $\mathrm{LaSrAlO}_{4}$. Physical Review B, 61(21):554-563, 2000.

[67] N. Banerjee, M. Huijben, G. Koster, and G. Rijnders. Direct patterning of functional interfaces in oxide heterostructures. Applied Physics Letters, 100(4):041601, 2012.

[68] Y. Chen, H.-J. Ko, S.-K. Hong, and T. Yao. Layer-by-layer growth of $\mathrm{ZnO}$ epilayer on $\mathrm{Al}_{2} \mathrm{O}_{3}$ (0001) by using a $\mathrm{MgO}$ buffer layer. Applied Physics Letters, 76(5):559, 2000.

[69] G Grüner. The dynamics of charge-density waves. Reviews of modern physics, 60(4):1129-1181, 1988.

[70] S. Hühn. Präparation und Eigenschaften von dünnen LPCMO Schichten. Diplomarbeit, 2009.

[71] O. L Krivanek, M. F. Chisholm, V. Nicolosi, T. J. Pennycook, G. J. Corbin, N. Dellby, M. F. Murfitt, C. S. Own, Z. S. Szilagyi, M. P. Oxley, S. T. Pantelides, and S. J. Pennycook. Atom-by-atom structural and chemical analysis by annular dark-field electron microscopy. Nature, 464(7288):571-574, 2010.

[72] R. W. G. Wyckoff. Crystal structures. Krieger, 1964.

[73] V. Moshnyaga, L. Sudheendra, O. I. Lebedev, S. A. Köster, K. Gehrke, O. Shapoval, A. Belenchuk, B. Damaschke, G. Van Tendeloo, and K. Samwer. A-site ordering versus electronic inhomogeneity in colossally magnetoresistive manganite films. Physical Review Letters, 97 (September):1-4, 2006.

[74] J. Narayan and B. C. Larson. Domain epitaxy: A unified paradigm for thin film growth. Journal of Applied Physics, 93(1):278, 2003.

[75] N. Loshkareva, L. Nomerovannaya, E. Mostovshchikova, A. Makhnev, Yu. Sukhorukov, N. Solin, T. Arbuzova, S. Naumov, N. Kostromitina, 
A. Balbashov, and L. Rybina. Electronic structure and polarons in $\mathrm{CaMnO}_{3-\delta}$ single crystals: Optical data. Physical Review B, 70(22): 224406, December 2004.

[76] H. Fujishiro and M. Ikebe. Canted Antiferromagnetic Order and Large Magnetoresistance Effect in $\mathrm{La}_{1-x} \mathrm{Ca}_{x} \mathrm{MnO}_{3}, \mathrm{Pr}_{1-x} \mathrm{Ca}_{x} \mathrm{MnO}_{3}$ and other $R E_{1-x} A E_{x} M n O_{3}$ Manganese Oxides (X. Journal of the Physical Society of Japan, 69(6):1865-1871, 2000.

[77] JJ Neumeier and JL Cohn. Possible signatures of magnetic phase segregation in electron-doped antiferromagnetic $\mathrm{CaMnO}_{3}$. Physical Review B, 61(21):319-322, 2000.

[78] T. I. Arbuzova, I. B. Smolyak, S. V. Naumov, A. A. Samokhvalov, and A. V. Korolev. Separation of Magnetic Phases in $\mathrm{La}_{1-x} \mathrm{Ca}_{x} \mathrm{MnO}_{3}$ Manganites with $0.6 \leq x \leq 0.9$. Journal of Experimental and Theoretical Physics, 92(1):100-107, 2001.

[79] C. Martin, A. Maignan, M. Hervieu, and B. Raveau. Magnetic phase diagrams of $L_{1-x} A_{x} M n O_{3}$ manganites $(\mathrm{L}=\mathrm{Pr}, \mathrm{Sm} ; \mathrm{A}=\mathrm{Ca}, \mathrm{Sr})$. Physical Review B, 60(17):191-199, 1999.

[80] L. Sudheendra, A. R. Raju, and C. N. R. Rao. A systematic study of four series of electron-doped rare earth manganates, $\mathrm{Ln}_{x} \mathrm{Ca}_{1-x} \mathrm{MnO}_{3}$ $(\mathrm{Ln}=\mathrm{La}, \mathrm{Nd}, \mathrm{Gd}$ and $\mathrm{Y}$ ) over the $\mathrm{x}=0.02-0.25$ composition range. Journal of Physics: Condensed Matter, 15:895-905, 2003.

[81] B. Gorshunov, E. Zhukova, V. I. Torgashev, L. S. Kadyrov, E. A. Motovilova, F. Fischgrabe, V. Moshnyaga, T. Zhang, R. Kremer, U. Pracht, S. Zapf, and M. Dressel. Boson peak in overdoped manganites $\mathrm{La}_{1-x} \mathrm{Ca} \mathrm{a}_{x} \mathrm{MnO}_{3}$. Physical Review B, 87(24):245124, June 2013.

[82] M. Iliev, M. Abrashev, J. Laverdière, S. Jandl, M. Gospodinov, Y.-Q. Wang, and Y.-Y. Sun. Distortion-dependent Raman spectra and mode mixing in $\mathrm{RMnO}_{3}$ perovskites $(\mathrm{R}=\mathrm{La}, \mathrm{Pr}, \mathrm{Nd}, \mathrm{Sm}, \mathrm{Eu}, \mathrm{Gd}, \mathrm{Tb}, \mathrm{Dy}, \mathrm{Ho}, \mathrm{Y})$. Physical Review B, 73(6):064302, February 2006.

[83] M. Abrashev, J. Bäckström, L. Börjesson, V. Popov, R. Chakalov, N. Kolev, R.-L. Meng, and M. Iliev. Raman spectroscopy of $\mathrm{CaMnO}_{3}$ : Mode assignment and relationship between Raman line intensities and structural distortions. Physical Review B, 65(18):184301, April 2002. 
[84] V. B. Podobedov, A. Weber, and D. B. Romero. Effect of structural and magnetic transitions in $\mathrm{La}_{1-x} \mathrm{M}_{x} \mathrm{MnO}_{3}(\mathrm{M}=\mathrm{Sr}, \mathrm{Ca})$ single crystals in Raman scattering. Physical Review B, 58(1):43-46, 1998.

[85] AE Pantoja and HJ Trodahl. Raman spectroscopy of orthorhombic $L a_{1-x} \mathrm{Ca}_{x} \mathrm{MnO}_{3}, \mathrm{x}=0.1-0.3$. Journal of Physics: Condensed matter, 13:3741-3752, 2001.

[86] S. Yoon, H. Liu, G. Schollerer, S. Cooper, P. Han, D. Payne, S.-W. Cheong, and Z. Fisk. Raman and optical spectroscopic studies of smallto-large polaron crossover in the perovskite manganese oxides. Physical Review B, 58(5):2795-2801, August 1998.

[87] A. Zawadowski and M. Cardona. Theory of Raman scattering on normal metals with impurities. Physical Review B, 42(16):10732-10734, December 1990.

[88] M. Iliev, M. N., Zhang, P. X., Habermeier, H.-U., Cardona. Raman spectroscopy as analytical tool for the local structure of $\mathrm{YBa}_{2} \mathrm{Cu}_{3} \mathrm{O}_{x}$ thin films. Journal of Alloys and Compounds, 251:99-102, 1997.

[89] V. B. Podobedov, D. B. Romero, a. Weber, J. P. Rice, R. Schreekala, M. Rajeswari, R. Ramesh, T. Venkatesan, and H. D. Drew. Diagnostics of colossal magnetoresistance manganite films by Raman spectroscopy. Applied Physics Letters, 73(22):3217, 1998.

[90] M. Iliev, A. Litvinchuk, H.-G. Lee, C. Chu, a. Barry, and J. Coey. Raman spectroscopy of ferromagnetic CrO2. Physical Review B, 60 (1):33-36, 1999.

[91] J. Laverdière, S. Jandl, A. A. Mukhin, V. Yu Ivanov, V. G. Ivanov, and M. N. Iliev. Spin-phonon coupling in orthorhombic $\mathrm{RMnO}_{3}$ $(\mathrm{R}=\mathrm{Pr}, \mathrm{Nd}, \mathrm{Sm}, \mathrm{Eu}, \mathrm{Gd}, \mathrm{Tb}, \mathrm{Dy}, \mathrm{Ho}, \mathrm{Y}):$ A Raman study. Physical Review B - Condensed Matter and Materials Physics, 73:1-5, 2006.

[92] E. Liarokapis, T. Leventouri, and D. Lampakis. Local lattice distortions and Raman spectra in the $L a_{1-x} \mathrm{Ca}_{x} M n \mathrm{O}_{3}$ system. Physical Review B, 60(18):758-763, 1999.

[93] J. Zuo, C. Xu, and Y. Liu. Crystallite size effects on the Raman spectra of $\mathrm{Mn}_{3} \mathrm{O}_{4}$. Nanostructured Materials, 10(8):1331-1335, 1998. 
[94] M. N. Iliev, H.-G. Lee, V. N. Popov, Y. Y. Sun, C. Thomsen, R. L. Meng, and C. W. Chu. Raman spectroscopy of orthorhombic perovskitelike $\mathrm{YMnO}_{3}$ and $\mathrm{LaMnO}_{3}$. Physical Review B, 57(5):2872-2877, February 1998.

[95] S. Turner, O. I. Lebedev, J. Verbeeck, K. Gehrke, V. Moshnyaga, and G. van Tendeloo. Structural phase transition and spontaneous interface reconstruction in $\mathrm{La}_{2 / 3} \mathrm{Ca}_{1 / 3} \mathrm{MnO}_{3} / \mathrm{BaTiO}_{3}$ superlattices. Physical Review B, 035418(87):1-8, 2013.

[96] T. Zhang, T. Zhou, T. Qian, and X. Li. Particle size effects on interplay between charge ordering and magnetic properties in nanosized $L a_{0.25} \mathrm{Ca}_{0.75} \mathrm{MnO}_{3}$. Physical Review B, 76(17):174415, November 2007.

[97] V. Markovich, I. Fita, A. Wisniewski, D. Mogilyansky, R. Puzniak, L. Titelman, and G. Gorodetsky. Size-driven magnetic transitions in $\mathrm{La}_{1 / 3} \mathrm{Ca}_{2 / 3} \mathrm{MnO}_{3}$ nanoparticles. Journal of Applied Physics, 108 (2010):063918, 2010.

[98] C. L. Lu, S. Dong, K. F. Wang, F. Gao, P. L. Li, L. Y. Lv, and J.-M. Liu. Charge-order breaking and ferromagnetism in $\mathrm{La}_{0.4} \mathrm{Ca}_{0.6} \mathrm{MnO}_{3}$ nanoparticles. Applied Physics Letters, 91(2007):032502, 2007.

[99] D. Niebieskikwiat and M. B. Salamon. Intrinsic interface exchange coupling of ferromagnetic nanodomains in a charge ordered manganite. Physical Review B - Condensed Matter and Materials Physics, 72:1-6, 2005 .

[100] X. G. Li, R. K. Zheng, G. Li, H. D. Zhou, R. X. Huang, J. Q. Xie, and Z. D. Wang. Jahn-Teller effect and stability of the charge-ordered state in $\mathrm{La}_{1-x} \mathrm{Ca}_{x} \mathrm{MnO} \mathrm{O}_{3}(0.5 \leq x \leq 0.9)$ manganite. Europhysics Letters, 60 (5), 2002. 


\section{Appendix}

Für die (001) Ebene können nach [46,63 sechs Zwillingsvarianten definiert werden. Deren Einheitsvektoren projiziert auf die Observationsebene lauten: Zwilling 1:

$$
x=\left(\begin{array}{c}
\frac{1}{\sqrt{2}} \\
0 \\
-\frac{1}{\sqrt{2}}
\end{array}\right) y=\left(\begin{array}{c}
\frac{1}{\sqrt{2}} \\
0 \\
\frac{1}{\sqrt{2}}
\end{array}\right) x^{\prime}=\left(\begin{array}{l}
1 \\
0 \\
0
\end{array}\right) y^{\prime}=\left(\begin{array}{l}
0 \\
0 \\
1
\end{array}\right)
$$

Zwilling 2:

$$
x=\left(\begin{array}{c}
-\frac{1}{\sqrt{2}} \\
0 \\
-\frac{1}{\sqrt{2}}
\end{array}\right) y=\left(\begin{array}{c}
\frac{1}{\sqrt{2}} \\
0 \\
-\frac{1}{\sqrt{2}}
\end{array}\right) x^{\prime}=\left(\begin{array}{c}
0 \\
0 \\
-1
\end{array}\right) y^{\prime}=\left(\begin{array}{l}
1 \\
0 \\
0
\end{array}\right)
$$

Zwilling 3:

$$
x=\left(\begin{array}{c}
\frac{1}{\sqrt{2}} \\
0 \\
-\frac{1}{\sqrt{2}}
\end{array}\right) y=\left(\begin{array}{l}
0 \\
1 \\
0
\end{array}\right) x^{\prime}=\left(\begin{array}{c}
\frac{1}{2} \\
\frac{1}{\sqrt{2}} \\
-\frac{1}{2}
\end{array}\right) y^{\prime}=\left(\begin{array}{c}
-\frac{1}{2} \\
\frac{1}{\sqrt{2}} \\
\frac{1}{2}
\end{array}\right)
$$

Zwilling 4:

$$
x=\left(\begin{array}{c}
-\frac{1}{\sqrt{2}} \\
0 \\
-\frac{1}{\sqrt{2}}
\end{array}\right) y=\left(\begin{array}{l}
0 \\
1 \\
0
\end{array}\right) x^{\prime}=\left(\begin{array}{c}
-\frac{1}{2} \\
\frac{1}{\sqrt{2}} \\
-\frac{1}{2}
\end{array}\right) y^{\prime}=\left(\begin{array}{c}
\frac{1}{2} \\
\frac{1}{\sqrt{2}} \\
\frac{1}{2}
\end{array}\right)
$$

Zwilling 5:

$$
x=\left(\begin{array}{l}
0 \\
1 \\
0
\end{array}\right) y=\left(\begin{array}{c}
-\frac{1}{\sqrt{2}} \\
0 \\
\frac{1}{\sqrt{2}}
\end{array}\right) x^{\prime}=\left(\begin{array}{c}
-\frac{1}{2} \\
\frac{1}{\sqrt{2}} \\
\frac{1}{2}
\end{array}\right) y^{\prime}=\left(\begin{array}{c}
-\frac{1}{2} \\
-\frac{1}{\sqrt{2}} \\
\frac{1}{2}
\end{array}\right)
$$

Zwilling 6:

$$
x=\left(\begin{array}{l}
0 \\
1 \\
0
\end{array}\right) y=\left(\begin{array}{c}
\frac{1}{\sqrt{2}} \\
0 \\
\frac{1}{\sqrt{2}}
\end{array}\right) x^{\prime}=\left(\begin{array}{c}
\frac{1}{2} \\
\frac{1}{\sqrt{2}} \\
\frac{1}{2}
\end{array}\right) y^{\prime}=\left(\begin{array}{c}
\frac{1}{2} \\
-\frac{1}{\sqrt{2}} \\
\frac{1}{2}
\end{array}\right)
$$

Damit ergeben sich mit $I_{\text {Raman }}=A \cdot\left[\sum e_{i}^{\sigma} R_{\rho \sigma} e_{s}^{\rho}\right]^{2}$ folgende Intensitäten:

XX Konfiguration $A_{g}$ Mode:

$$
A_{g}=\left(\begin{array}{lll}
\frac{1}{\sqrt{2}} & 0 & -\frac{1}{\sqrt{2}}
\end{array}\right) \cdot\left(\begin{array}{lll}
a & 0 & 0 \\
0 & b & 0 \\
0 & 0 & c
\end{array}\right) \cdot\left(\begin{array}{c}
\frac{1}{\sqrt{2}} \\
0 \\
-\frac{1}{\sqrt{2}}
\end{array}\right)=\frac{1}{2}(a+c)
$$




$$
\begin{gathered}
A_{g}=\left(\begin{array}{lll}
-\frac{1}{\sqrt{2}} & 0 & -\frac{1}{\sqrt{2}}
\end{array}\right) \cdot\left(\begin{array}{lll}
a & 0 & 0 \\
0 & b & 0 \\
0 & 0 & c
\end{array}\right) \cdot\left(\begin{array}{c}
-\frac{1}{\sqrt{2}} \\
0 \\
-\frac{1}{\sqrt{2}}
\end{array}\right)=\frac{1}{2}(a+c) \\
A_{g}=\left(\begin{array}{lll}
\frac{1}{\sqrt{2}} & 0 & -\frac{1}{\sqrt{2}}
\end{array}\right) \cdot\left(\begin{array}{lll}
a & 0 & 0 \\
0 & b & 0 \\
0 & 0 & c
\end{array}\right) \cdot\left(\begin{array}{c}
\frac{1}{\sqrt{2}} \\
0 \\
-\frac{1}{\sqrt{2}}
\end{array}\right)=\frac{1}{2}(a+c) \\
A_{g}=\left(\begin{array}{lll}
-\frac{1}{\sqrt{2}} & 0 & -\frac{1}{\sqrt{2}}
\end{array}\right) \cdot\left(\begin{array}{lll}
a & 0 & 0 \\
0 & b & 0 \\
0 & 0 & c
\end{array}\right) \cdot\left(\begin{array}{c}
-\frac{1}{\sqrt{2}} \\
0 \\
-\frac{1}{\sqrt{2}}
\end{array}\right)=\frac{1}{2}(a+c) \\
A_{g}=\left(\begin{array}{lll}
0 & 1 & 0
\end{array}\right) \cdot\left(\begin{array}{lll}
a & 0 & 0 \\
0 & b & 0 \\
0 & 0 & c
\end{array}\right) \cdot\left(\begin{array}{l}
0 \\
1 \\
0
\end{array}\right)=b \\
A_{g}=\left(\begin{array}{lll}
0 & 1 & 0
\end{array}\right) \cdot\left(\begin{array}{lll}
a & 0 & 0 \\
0 & b & 0 \\
0 & 0 & c
\end{array}\right) \cdot\left(\begin{array}{l}
0 \\
1 \\
0
\end{array}\right)=b
\end{gathered}
$$

XY Konfiguration $A_{g}$ Mode:

$$
\begin{gathered}
A_{g}=\left(\begin{array}{lll}
\frac{1}{\sqrt{2}} & 0 & -\frac{1}{\sqrt{2}}
\end{array}\right) \cdot\left(\begin{array}{lll}
a & 0 & 0 \\
0 & b & 0 \\
0 & 0 & c
\end{array}\right) \cdot\left(\begin{array}{c}
\frac{1}{\sqrt{2}} \\
0 \\
\frac{1}{\sqrt{2}}
\end{array}\right)=\frac{1}{2}(a-c) \\
A_{g}=\left(\begin{array}{lll}
-\frac{1}{\sqrt{2}} & 0 & -\frac{1}{\sqrt{2}}
\end{array}\right) \cdot\left(\begin{array}{lll}
a & 0 & 0 \\
0 & b & 0 \\
0 & 0 & c
\end{array}\right) \cdot\left(\begin{array}{c}
\frac{1}{\sqrt{2}} \\
0 \\
-\frac{1}{\sqrt{2}}
\end{array}\right)=\frac{1}{2}(a-c) \\
A_{g}=\left(\begin{array}{lll}
\frac{1}{\sqrt{2}} & 0 & -\frac{1}{\sqrt{2}}
\end{array}\right) \cdot\left(\begin{array}{lll}
a & 0 & 0 \\
0 & b & 0 \\
0 & 0 & c
\end{array}\right) \cdot\left(\begin{array}{l}
0 \\
1 \\
0
\end{array}\right)=0 \\
A_{g}=\left(\begin{array}{lll}
-\frac{1}{\sqrt{2}} & 0 & -\frac{1}{\sqrt{2}}
\end{array}\right) \cdot\left(\begin{array}{lll}
a & 0 & 0 \\
0 & b & 0 \\
0 & 0 & c
\end{array}\right) \cdot\left(\begin{array}{l}
0 \\
1 \\
0
\end{array}\right)=0 \\
A_{g}=\left(\begin{array}{lll}
0 & 1 & 0
\end{array}\right) \cdot\left(\begin{array}{lll}
a & 0 & 0 \\
0 & b & 0 \\
0 & 0 & c
\end{array}\right) \cdot\left(\begin{array}{c}
-\frac{1}{\sqrt{2}} \\
0 \\
\frac{1}{\sqrt{2}}
\end{array}\right)=0 \\
A_{g}=\left(\begin{array}{lll}
0 & 1 & 0
\end{array}\right) \cdot\left(\begin{array}{lll}
a & 0 & 0 \\
0 & b & 0 \\
0 & 0 & c
\end{array}\right) \cdot\left(\begin{array}{c}
\frac{1}{\sqrt{2}} \\
0 \\
\frac{1}{\sqrt{2}}
\end{array}\right)=0
\end{gathered}
$$


X'X' Konfiguration $A_{g}$ Mode:

$$
\begin{gathered}
A_{g}=\left(\begin{array}{lll}
1 & 0 & 0
\end{array}\right) \cdot\left(\begin{array}{lll}
a & 0 & 0 \\
0 & b & 0 \\
0 & 0 & c
\end{array}\right) \cdot\left(\begin{array}{l}
1 \\
0 \\
0
\end{array}\right)=a \\
A_{g}=\left(\begin{array}{lll}
0 & 0 & -1
\end{array}\right) \cdot\left(\begin{array}{lll}
a & 0 & 0 \\
0 & b & 0 \\
0 & 0 & c
\end{array}\right) \cdot\left(\begin{array}{c}
0 \\
0 \\
-1
\end{array}\right)=c \\
A_{g}=\left(\begin{array}{lll}
\frac{1}{2} & \frac{1}{\sqrt{2}} & -\frac{1}{2}
\end{array}\right) \cdot\left(\begin{array}{lll}
a & 0 & 0 \\
0 & b & 0 \\
0 & 0 & c
\end{array}\right) \cdot\left(\begin{array}{c}
\frac{1}{2} \\
\frac{1}{\sqrt{2}} \\
-\frac{1}{2}
\end{array}\right)=\frac{1}{4}(a+2 b+c) \\
A_{g}=\left(\begin{array}{lll}
-\frac{1}{2} & \frac{1}{\sqrt{2}} & -\frac{1}{2}
\end{array}\right) \cdot\left(\begin{array}{lll}
a & 0 & 0 \\
0 & b & 0 \\
0 & 0 & c
\end{array}\right) \cdot\left(\begin{array}{c}
-\frac{1}{2} \\
\frac{1}{\sqrt{2}} \\
-\frac{1}{2}
\end{array}\right)=\frac{1}{4}(a+2 b+c) \\
A_{g}=\left(\begin{array}{lll}
-\frac{1}{2} & \frac{1}{\sqrt{2}} & \frac{1}{2}
\end{array}\right) \cdot\left(\begin{array}{lll}
a & 0 & 0 \\
0 & b & 0 \\
0 & 0 & c
\end{array}\right) \cdot\left(\begin{array}{c}
-\frac{1}{2} \\
\frac{1}{\sqrt{2}} \\
\frac{1}{2}
\end{array}\right)=\frac{1}{4}(a+2 b+c) \\
A_{g}=\left(\begin{array}{lll}
\frac{1}{2} & \frac{1}{\sqrt{2}} & \frac{1}{2}
\end{array}\right) \cdot\left(\begin{array}{lll}
a & 0 & 0 \\
0 & b & 0 \\
0 & 0 & c
\end{array}\right) \cdot\left(\begin{array}{c}
\frac{1}{2} \\
\frac{1}{\sqrt{2}} \\
\frac{1}{2}
\end{array}\right)=\frac{1}{4}(a+2 b+c)
\end{gathered}
$$

X'Y' Konfiguration $A_{g}$ Mode:

$$
\begin{gathered}
A_{g}=\left(\begin{array}{lll}
1 & 0 & 0
\end{array}\right) \cdot\left(\begin{array}{lll}
a & 0 & 0 \\
0 & b & 0 \\
0 & 0 & c
\end{array}\right) \cdot\left(\begin{array}{l}
0 \\
0 \\
1
\end{array}\right)=0 \\
A_{g}=\left(\begin{array}{lll}
0 & 0 & -1
\end{array}\right) \cdot\left(\begin{array}{lll}
a & 0 & 0 \\
0 & b & 0 \\
0 & 0 & c
\end{array}\right) \cdot\left(\begin{array}{l}
1 \\
0 \\
0
\end{array}\right)=0 \\
A_{g}=\left(\begin{array}{lll}
\frac{1}{2} & \frac{1}{\sqrt{2}} & -\frac{1}{2}
\end{array}\right) \cdot\left(\begin{array}{lll}
a & 0 & 0 \\
0 & b & 0 \\
0 & 0 & c
\end{array}\right) \cdot\left(\begin{array}{c}
-\frac{1}{2} \\
\frac{1}{\sqrt{2}} \\
\frac{1}{2}
\end{array}\right)=\frac{1}{4}(-a+2 b-c) \\
A_{g}=\left(\begin{array}{lll}
-\frac{1}{2} & \frac{1}{\sqrt{2}} & -\frac{1}{2}
\end{array}\right) \cdot\left(\begin{array}{lll}
a & 0 & 0 \\
0 & b & 0 \\
0 & 0 & c
\end{array}\right) \cdot\left(\begin{array}{c}
\frac{1}{2} \\
\frac{1}{\sqrt{2}} \\
\frac{1}{2}
\end{array}\right)=\frac{1}{4}(-a+2 b-c)
\end{gathered}
$$




$$
\begin{aligned}
& A_{g}=\left(\begin{array}{lll}
-\frac{1}{2} & \frac{1}{\sqrt{2}} & \frac{1}{2}
\end{array}\right) \cdot\left(\begin{array}{lll}
a & 0 & 0 \\
0 & b & 0 \\
0 & 0 & c
\end{array}\right) \cdot\left(\begin{array}{c}
-\frac{1}{2} \\
-\frac{1}{\sqrt{2}} \\
\frac{1}{2}
\end{array}\right)=\frac{1}{4}(-a+2 b-c) \\
& A_{g}=\left(\begin{array}{lll}
\frac{1}{2} & \frac{1}{\sqrt{2}} & \frac{1}{2}
\end{array}\right) \cdot\left(\begin{array}{lll}
a & 0 & 0 \\
0 & b & 0 \\
0 & 0 & c
\end{array}\right) \cdot\left(\begin{array}{c}
\frac{1}{2} \\
-\frac{1}{\sqrt{2}} \\
\frac{1}{2}
\end{array}\right)=\frac{1}{4}(-a+2 b-c)
\end{aligned}
$$

XX Konfiguration $B_{1 g}$ Mode:

$$
\begin{gathered}
B_{1 g}=\left(\begin{array}{lll}
\frac{1}{\sqrt{2}} & 0 & -\frac{1}{\sqrt{2}}
\end{array}\right) \cdot\left(\begin{array}{lll}
0 & d & 0 \\
d & 0 & 0 \\
0 & 0 & 0
\end{array}\right) \cdot\left(\begin{array}{c}
\frac{1}{\sqrt{2}} \\
0 \\
-\frac{1}{\sqrt{2}}
\end{array}\right)=0 \\
B_{1 g}=\left(\begin{array}{lll}
-\frac{1}{\sqrt{2}} & 0 & -\frac{1}{\sqrt{2}}
\end{array}\right) \cdot\left(\begin{array}{lll}
0 & d & 0 \\
d & 0 & 0 \\
0 & 0 & 0
\end{array}\right) \cdot\left(\begin{array}{c}
-\frac{1}{\sqrt{2}} \\
0 \\
-\frac{1}{\sqrt{2}}
\end{array}\right)=0 \\
B_{1 g}=\left(\begin{array}{lll}
\frac{1}{\sqrt{2}} & 0 & -\frac{1}{\sqrt{2}}
\end{array}\right) \cdot\left(\begin{array}{lll}
0 & d & 0 \\
d & 0 & 0 \\
0 & 0 & 0
\end{array}\right) \cdot\left(\begin{array}{c}
\frac{1}{\sqrt{2}} \\
0 \\
-\frac{1}{\sqrt{2}}
\end{array}\right)=0 \\
B_{1 g}=\left(\begin{array}{lll}
-\frac{1}{\sqrt{2}} & 0 & -\frac{1}{\sqrt{2}}
\end{array}\right) \cdot\left(\begin{array}{lll}
0 & d & 0 \\
d & 0 & 0 \\
0 & 0 & 0
\end{array}\right) \cdot\left(\begin{array}{c}
-\frac{1}{\sqrt{2}} \\
0 \\
-\frac{1}{\sqrt{2}}
\end{array}\right)=0 \\
B_{1 g}=\left(\begin{array}{lll}
0 & 1 & 0
\end{array}\right) \cdot\left(\begin{array}{lll}
0 & d & 0 \\
d & 0 & 0 \\
0 & 0 & 0
\end{array}\right) \cdot\left(\begin{array}{l}
0 \\
1 \\
0
\end{array}\right)=0 \\
B_{1 g}=\left(\begin{array}{lll}
0 & 1 & 0
\end{array}\right) \cdot\left(\begin{array}{lll}
0 & d & 0 \\
d & 0 & 0 \\
0 & 0 & 0
\end{array}\right) \cdot\left(\begin{array}{l}
0 \\
1 \\
0
\end{array}\right)=0
\end{gathered}
$$

XY Konfiguration $B_{1 g}$ Mode:

$$
\begin{gathered}
B_{1 g}=\left(\begin{array}{lll}
\frac{1}{\sqrt{2}} & 0 & -\frac{1}{\sqrt{2}}
\end{array}\right) \cdot\left(\begin{array}{lll}
0 & d & 0 \\
d & 0 & 0 \\
0 & 0 & 0
\end{array}\right) \cdot\left(\begin{array}{c}
\frac{1}{\sqrt{2}} \\
0 \\
\frac{1}{\sqrt{2}}
\end{array}\right)=0 \\
B_{1 g}=\left(\begin{array}{lll}
-\frac{1}{\sqrt{2}} & 0 & -\frac{1}{\sqrt{2}}
\end{array}\right) \cdot\left(\begin{array}{lll}
0 & d & 0 \\
d & 0 & 0 \\
0 & 0 & 0
\end{array}\right) \cdot\left(\begin{array}{c}
\frac{1}{\sqrt{2}} \\
0 \\
-\frac{1}{\sqrt{2}}
\end{array}\right)=0
\end{gathered}
$$




$$
\begin{gathered}
B_{1 g}=\left(\begin{array}{lll}
\frac{1}{\sqrt{2}} & 0 & -\frac{1}{\sqrt{2}}
\end{array}\right) \cdot\left(\begin{array}{lll}
0 & d & 0 \\
d & 0 & 0 \\
0 & 0 & 0
\end{array}\right) \cdot\left(\begin{array}{l}
0 \\
1 \\
0
\end{array}\right)=\frac{1}{\sqrt{2}} \cdot d \\
B_{1 g}=\left(\begin{array}{lll}
-\frac{1}{\sqrt{2}} & 0 & -\frac{1}{\sqrt{2}}
\end{array}\right) \cdot\left(\begin{array}{lll}
0 & d & 0 \\
d & 0 & 0 \\
0 & 0 & 0
\end{array}\right) \cdot\left(\begin{array}{l}
0 \\
1 \\
0
\end{array}\right)=\frac{1}{\sqrt{2}} \cdot d \\
B_{1 g}=\left(\begin{array}{lll}
0 & 1 & 0
\end{array}\right) \cdot\left(\begin{array}{lll}
0 & d & 0 \\
d & 0 & 0 \\
0 & 0 & 0
\end{array}\right) \cdot\left(\begin{array}{c}
-\frac{1}{\sqrt{2}} \\
0 \\
\frac{1}{\sqrt{2}}
\end{array}\right)=\frac{1}{\sqrt{2}} \cdot d \\
B_{1 g}=\left(\begin{array}{lll}
0 & 1 & 0
\end{array}\right) \cdot\left(\begin{array}{lll}
0 & d & 0 \\
d & 0 & 0 \\
0 & 0 & 0
\end{array}\right) \cdot\left(\begin{array}{c}
\frac{1}{\sqrt{2}} \\
0 \\
\frac{1}{\sqrt{2}}
\end{array}\right)=\frac{1}{\sqrt{2}} \cdot d
\end{gathered}
$$

X'X' Konfiguration $B_{1 g}$ Mode:

$$
\begin{gathered}
B_{1 g}=\left(\begin{array}{lll}
1 & 0 & 0
\end{array}\right) \cdot\left(\begin{array}{lll}
0 & d & 0 \\
d & 0 & 0 \\
0 & 0 & 0
\end{array}\right) \cdot\left(\begin{array}{c}
1 \\
0 \\
0
\end{array}\right)=0 \\
B_{1 g}=\left(\begin{array}{lll}
0 & 0 & -1
\end{array}\right) \cdot\left(\begin{array}{lll}
0 & d & 0 \\
d & 0 & 0 \\
0 & 0 & 0
\end{array}\right) \cdot\left(\begin{array}{c}
0 \\
0 \\
-1
\end{array}\right)=0 \\
B_{1 g}=\left(\begin{array}{lll}
\frac{1}{2} & \frac{1}{\sqrt{2}} & -\frac{1}{2}
\end{array}\right) \cdot\left(\begin{array}{lll}
0 & d & 0 \\
d & 0 & 0 \\
0 & 0 & 0
\end{array}\right) \cdot\left(\begin{array}{c}
\frac{1}{2} \\
\frac{1}{\sqrt{2}} \\
-\frac{1}{2}
\end{array}\right)=\frac{1}{\sqrt{2}} \cdot d \\
B_{1 g}=\left(\begin{array}{lll}
-\frac{1}{2} & \frac{1}{\sqrt{2}} & -\frac{1}{2}
\end{array}\right) \cdot\left(\begin{array}{lll}
0 & d & 0 \\
d & 0 & 0 \\
0 & 0 & 0
\end{array}\right) \cdot\left(\begin{array}{c}
-\frac{1}{2} \\
\frac{1}{\sqrt{2}} \\
-\frac{1}{2}
\end{array}\right)=\frac{1}{\sqrt{2}} \cdot d \\
B_{1 g}=\left(\begin{array}{lll}
-\frac{1}{2} & \frac{1}{\sqrt{2}} & \frac{1}{2}
\end{array}\right) \cdot\left(\begin{array}{lll}
0 & d & 0 \\
d & 0 & 0 \\
0 & 0 & 0
\end{array}\right) \cdot\left(\begin{array}{c}
-\frac{1}{2} \\
\frac{1}{\sqrt{2}} \\
\frac{1}{2}
\end{array}\right)=\frac{1}{\sqrt{2}} \cdot d \\
B_{1 g}=\left(\begin{array}{lll}
\frac{1}{2} & \frac{1}{\sqrt{2}} & \frac{1}{2}
\end{array}\right) \cdot\left(\begin{array}{lll}
0 & d & 0 \\
d & 0 & 0 \\
0 & 0 & 0
\end{array}\right) \cdot\left(\begin{array}{c}
\frac{1}{2} \\
\frac{1}{\sqrt{2}} \\
\frac{1}{2}
\end{array}\right)=\frac{1}{\sqrt{2}} \cdot d
\end{gathered}
$$

X'Y' Konfiguration $B_{1 g}$ Mode:

$$
B_{1 g}=\left(\begin{array}{lll}
1 & 0 & 0
\end{array}\right) \cdot\left(\begin{array}{lll}
0 & d & 0 \\
d & 0 & 0 \\
0 & 0 & 0
\end{array}\right) \cdot\left(\begin{array}{l}
0 \\
0 \\
1
\end{array}\right)=0
$$




$$
\begin{gathered}
B_{1 g}=\left(\begin{array}{lll}
0 & 0 & -1
\end{array}\right) \cdot\left(\begin{array}{lll}
0 & d & 0 \\
d & 0 & 0 \\
0 & 0 & 0
\end{array}\right) \cdot\left(\begin{array}{c}
1 \\
0 \\
0
\end{array}\right)=0 \\
B_{1 g}=\left(\begin{array}{lll}
\frac{1}{2} & \frac{1}{\sqrt{2}} & -\frac{1}{2}
\end{array}\right) \cdot\left(\begin{array}{lll}
0 & d & 0 \\
d & 0 & 0 \\
0 & 0 & 0
\end{array}\right) \cdot\left(\begin{array}{c}
-\frac{1}{2} \\
\frac{1}{\sqrt{2}} \\
\frac{1}{2}
\end{array}\right)=0 \\
B_{1 g}=\left(\begin{array}{lll}
-\frac{1}{2} & \frac{1}{\sqrt{2}} & -\frac{1}{2}
\end{array}\right) \cdot\left(\begin{array}{lll}
0 & d & 0 \\
d & 0 & 0 \\
0 & 0 & 0
\end{array}\right) \cdot\left(\begin{array}{c}
\frac{1}{2} \\
\frac{1}{\sqrt{2}} \\
\frac{1}{2}
\end{array}\right)=0 \\
B_{1 g}=\left(\begin{array}{lll}
-\frac{1}{2} & \frac{1}{\sqrt{2}} & \frac{1}{2}
\end{array}\right) \cdot\left(\begin{array}{lll}
0 & d & 0 \\
d & 0 & 0 \\
0 & 0 & 0
\end{array}\right) \cdot\left(\begin{array}{c}
-\frac{1}{2} \\
-\frac{1}{\sqrt{2}} \\
\frac{1}{2}
\end{array}\right)=0 \\
B_{1 g}=\left(\begin{array}{lll}
\frac{1}{2} & \frac{1}{\sqrt{2}} & \frac{1}{2}
\end{array}\right) \cdot\left(\begin{array}{lll}
0 & d & 0 \\
d & 0 & 0 \\
0 & 0 & 0
\end{array}\right) \cdot\left(\begin{array}{c}
\frac{1}{2} \\
-\frac{1}{\sqrt{2}} \\
\frac{1}{2}
\end{array}\right)=0
\end{gathered}
$$

XX Konfiguration $B_{2 g}$ Mode:

$$
\begin{gathered}
B_{2 g}=\left(\begin{array}{lll}
\frac{1}{\sqrt{2}} & 0 & -\frac{1}{\sqrt{2}}
\end{array}\right) \cdot\left(\begin{array}{lll}
0 & 0 & e \\
0 & 0 & 0 \\
e & 0 & 0
\end{array}\right) \cdot\left(\begin{array}{c}
\frac{1}{\sqrt{2}} \\
0 \\
-\frac{1}{\sqrt{2}}
\end{array}\right)=e \\
B_{2 g}=\left(\begin{array}{lll}
-\frac{1}{\sqrt{2}} & 0 & -\frac{1}{\sqrt{2}}
\end{array}\right) \cdot\left(\begin{array}{lll}
0 & 0 & e \\
0 & 0 & 0 \\
e & 0 & 0
\end{array}\right) \cdot\left(\begin{array}{c}
-\frac{1}{\sqrt{2}} \\
0 \\
-\frac{1}{\sqrt{2}}
\end{array}\right)=e \\
B_{2 g}=\left(\begin{array}{lll}
\frac{1}{\sqrt{2}} & 0 & -\frac{1}{\sqrt{2}}
\end{array}\right) \cdot\left(\begin{array}{lll}
0 & 0 & e \\
0 & 0 & 0 \\
e & 0 & 0
\end{array}\right) \cdot\left(\begin{array}{c}
\frac{1}{\sqrt{2}} \\
0 \\
-\frac{1}{\sqrt{2}}
\end{array}\right)=e \\
B_{2 g}=\left(\begin{array}{lll}
-\frac{1}{\sqrt{2}} & 0 & -\frac{1}{\sqrt{2}}
\end{array}\right) \cdot\left(\begin{array}{lll}
0 & 0 & e \\
0 & 0 & 0 \\
e & 0 & 0
\end{array}\right) \cdot\left(\begin{array}{c}
-\frac{1}{\sqrt{2}} \\
0 \\
-\frac{1}{\sqrt{2}}
\end{array}\right)=e \\
B_{2 g}=\left(\begin{array}{lll}
0 & 1 & 0
\end{array}\right) \cdot\left(\begin{array}{lll}
0 & 0 & e \\
0 & 0 & 0 \\
e & 0 & 0
\end{array}\right) \cdot\left(\begin{array}{l}
0 \\
1 \\
0
\end{array}\right)=0 \\
B_{2 g}=\left(\begin{array}{lll}
0 & 1 & 0
\end{array}\right) \cdot\left(\begin{array}{lll}
0 & 0 & e \\
0 & 0 & 0 \\
e & 0 & 0
\end{array}\right) \cdot\left(\begin{array}{l}
0 \\
1 \\
0
\end{array}\right)=0
\end{gathered}
$$


XY Konfiguration $B_{2 g}$ Mode:

$$
\begin{gathered}
B_{2 g}=\left(\begin{array}{lll}
\frac{1}{\sqrt{2}} & 0 & -\frac{1}{\sqrt{2}}
\end{array}\right) \cdot\left(\begin{array}{lll}
0 & 0 & e \\
0 & 0 & 0 \\
e & 0 & 0
\end{array}\right) \cdot\left(\begin{array}{c}
\frac{1}{\sqrt{2}} \\
0 \\
\frac{1}{\sqrt{2}}
\end{array}\right)=0 \\
B_{2 g}=\left(\begin{array}{lll}
-\frac{1}{\sqrt{2}} & 0 & -\frac{1}{\sqrt{2}}
\end{array}\right) \cdot\left(\begin{array}{lll}
0 & 0 & e \\
0 & 0 & 0 \\
e & 0 & 0
\end{array}\right) \cdot\left(\begin{array}{c}
\frac{1}{\sqrt{2}} \\
0 \\
-\frac{1}{\sqrt{2}}
\end{array}\right)=0 \\
B_{2 g}=\left(\begin{array}{lll}
\frac{1}{\sqrt{2}} & 0 & -\frac{1}{\sqrt{2}}
\end{array}\right) \cdot\left(\begin{array}{lll}
0 & 0 & e \\
0 & 0 & 0 \\
e & 0 & 0
\end{array}\right) \cdot\left(\begin{array}{c}
0 \\
1 \\
0
\end{array}\right)=0 \\
B_{2 g}=\left(\begin{array}{lll}
-\frac{1}{\sqrt{2}} & 0 & -\frac{1}{\sqrt{2}}
\end{array}\right) \cdot\left(\begin{array}{lll}
0 & 0 & e \\
0 & 0 & 0 \\
e & 0 & 0
\end{array}\right) \cdot\left(\begin{array}{l}
0 \\
1 \\
0
\end{array}\right)=0 \\
B_{2 g}=\left(\begin{array}{lll}
0 & 1 & 0
\end{array}\right) \cdot\left(\begin{array}{lll}
0 & 0 & e \\
0 & 0 & 0 \\
e & 0 & 0
\end{array}\right) \cdot\left(\begin{array}{c}
-\frac{1}{\sqrt{2}} \\
0 \\
\frac{1}{\sqrt{2}}
\end{array}\right)=0 \\
B_{2 g}=\left(\begin{array}{lll}
0 & 1 & 0
\end{array}\right) \cdot\left(\begin{array}{lll}
0 & 0 & e \\
0 & 0 & 0 \\
e & 0 & 0
\end{array}\right) \cdot\left(\begin{array}{c}
\frac{1}{\sqrt{2}} \\
0 \\
\frac{1}{\sqrt{2}}
\end{array}\right)=0
\end{gathered}
$$

X'X' Konfiguration $B_{2 g}$ Mode:

$$
\begin{gathered}
B_{2 g}=\left(\begin{array}{lll}
1 & 0 & 0
\end{array}\right) \cdot\left(\begin{array}{lll}
0 & 0 & e \\
0 & 0 & 0 \\
e & 0 & 0
\end{array}\right) \cdot\left(\begin{array}{c}
1 \\
0 \\
0
\end{array}\right)=0 \\
B_{2 g}=\left(\begin{array}{lll}
0 & 0 & -1
\end{array}\right) \cdot\left(\begin{array}{lll}
0 & 0 & e \\
0 & 0 & 0 \\
e & 0 & 0
\end{array}\right) \cdot\left(\begin{array}{c}
0 \\
0 \\
-1
\end{array}\right)=0 \\
B_{2 g}=\left(\begin{array}{lll}
\frac{1}{2} & \frac{1}{\sqrt{2}} & -\frac{1}{2}
\end{array}\right) \cdot\left(\begin{array}{lll}
0 & 0 & e \\
0 & 0 & 0 \\
e & 0 & 0
\end{array}\right) \cdot\left(\begin{array}{c}
\frac{1}{2} \\
\frac{1}{\sqrt{2}} \\
-\frac{1}{2}
\end{array}\right)=\frac{1}{2} \cdot e \\
B_{2 g}=\left(\begin{array}{lll}
-\frac{1}{2} & \frac{1}{\sqrt{2}} & -\frac{1}{2}
\end{array}\right) \cdot\left(\begin{array}{lll}
0 & 0 & e \\
0 & 0 & 0 \\
e & 0 & 0
\end{array}\right) \cdot\left(\begin{array}{c}
-\frac{1}{2} \\
\frac{1}{\sqrt{2}} \\
-\frac{1}{2}
\end{array}\right)=\frac{1}{2} \cdot e
\end{gathered}
$$




$$
\begin{gathered}
B_{2 g}=\left(\begin{array}{lll}
-\frac{1}{2} & \frac{1}{\sqrt{2}} & \frac{1}{2}
\end{array}\right) \cdot\left(\begin{array}{lll}
0 & 0 & e \\
0 & 0 & 0 \\
e & 0 & 0
\end{array}\right) \cdot\left(\begin{array}{c}
-\frac{1}{2} \\
\frac{1}{\sqrt{2}} \\
\frac{1}{2}
\end{array}\right)=\frac{1}{2} \cdot e \\
B_{2 g}=\left(\begin{array}{lll}
\frac{1}{2} & \frac{1}{\sqrt{2}} & \frac{1}{2}
\end{array}\right) \cdot\left(\begin{array}{lll}
0 & 0 & e \\
0 & 0 & 0 \\
e & 0 & 0
\end{array}\right) \cdot\left(\begin{array}{c}
\frac{1}{2} \\
\frac{1}{\sqrt{2}} \\
\frac{1}{2}
\end{array}\right)=\frac{1}{2} \cdot e
\end{gathered}
$$

X'Y' Konfiguration $B_{2 g}$ Mode:

$$
\begin{gathered}
B_{2 g}=\left(\begin{array}{lll}
1 & 0 & 0
\end{array}\right) \cdot\left(\begin{array}{lll}
0 & 0 & e \\
0 & 0 & 0 \\
e & 0 & 0
\end{array}\right) \cdot\left(\begin{array}{l}
0 \\
0 \\
1
\end{array}\right)=e \\
B_{2 g}=\left(\begin{array}{lll}
0 & 0 & -1
\end{array}\right) \cdot\left(\begin{array}{lll}
0 & 0 & e \\
0 & 0 & 0 \\
e & 0 & 0
\end{array}\right) \cdot\left(\begin{array}{l}
1 \\
0 \\
0
\end{array}\right)=e \\
B_{2 g}=\left(\begin{array}{lll}
\frac{1}{2} & \frac{1}{\sqrt{2}} & -\frac{1}{2}
\end{array}\right) \cdot\left(\begin{array}{lll}
0 & 0 & e \\
0 & 0 & 0 \\
e & 0 & 0
\end{array}\right) \cdot\left(\begin{array}{c}
-\frac{1}{2} \\
\frac{1}{\sqrt{2}} \\
\frac{1}{2}
\end{array}\right)=\frac{1}{2} \cdot e \\
B_{2 g}=\left(\begin{array}{lll}
-\frac{1}{2} & \frac{1}{\sqrt{2}} & -\frac{1}{2}
\end{array}\right) \cdot\left(\begin{array}{lll}
0 & 0 & e \\
0 & 0 & 0 \\
e & 0 & 0
\end{array}\right) \cdot\left(\begin{array}{c}
\frac{1}{2} \\
\frac{1}{\sqrt{2}} \\
\frac{1}{2}
\end{array}\right)=\frac{1}{2} \cdot e \\
B_{2 g}=\left(\begin{array}{lll}
-\frac{1}{2} & \frac{1}{\sqrt{2}} & \frac{1}{2}
\end{array}\right) \cdot\left(\begin{array}{lll}
0 & 0 & e \\
0 & 0 & 0 \\
e & 0 & 0
\end{array}\right) \cdot\left(\begin{array}{c}
-\frac{1}{2} \\
-\frac{1}{\sqrt{2}} \\
\frac{1}{2}
\end{array}\right)=\frac{1}{2} \cdot e \\
B_{2 g}=\left(\begin{array}{lll}
\frac{1}{2} & \frac{1}{\sqrt{2}} & \frac{1}{2}
\end{array}\right) \cdot\left(\begin{array}{lll}
0 & 0 & e \\
0 & 0 & 0 \\
e & 0 & 0
\end{array}\right) \cdot\left(\begin{array}{c}
\frac{1}{2} \\
-\frac{1}{\sqrt{2}} \\
\frac{1}{2}
\end{array}\right)=\frac{1}{2} \cdot e
\end{gathered}
$$

XX Konfiguration $B_{3 g}$ Mode:

$$
\begin{gathered}
B_{3 g}=\left(\begin{array}{lll}
\frac{1}{\sqrt{2}} & 0 & -\frac{1}{\sqrt{2}}
\end{array}\right) \cdot\left(\begin{array}{lll}
0 & 0 & 0 \\
0 & 0 & f \\
0 & f & 0
\end{array}\right) \cdot\left(\begin{array}{c}
\frac{1}{\sqrt{2}} \\
0 \\
-\frac{1}{\sqrt{2}}
\end{array}\right)=0 \\
B_{3 g}=\left(\begin{array}{lll}
-\frac{1}{\sqrt{2}} & 0 & -\frac{1}{\sqrt{2}}
\end{array}\right) \cdot\left(\begin{array}{lll}
0 & 0 & 0 \\
0 & 0 & f \\
0 & f & 0
\end{array}\right) \cdot\left(\begin{array}{c}
-\frac{1}{\sqrt{2}} \\
0 \\
-\frac{1}{\sqrt{2}}
\end{array}\right)=0
\end{gathered}
$$




$$
\begin{gathered}
B_{3 g}=\left(\begin{array}{lll}
\frac{1}{\sqrt{2}} & 0 & -\frac{1}{\sqrt{2}}
\end{array}\right) \cdot\left(\begin{array}{lll}
0 & 0 & 0 \\
0 & 0 & f \\
0 & f & 0
\end{array}\right) \cdot\left(\begin{array}{c}
\frac{1}{\sqrt{2}} \\
0 \\
-\frac{1}{\sqrt{2}}
\end{array}\right)=0 \\
B_{3 g}=\left(\begin{array}{lll}
-\frac{1}{\sqrt{2}} & 0 & -\frac{1}{\sqrt{2}}
\end{array}\right) \cdot\left(\begin{array}{lll}
0 & 0 & 0 \\
0 & 0 & f \\
0 & f & 0
\end{array}\right) \cdot\left(\begin{array}{c}
-\frac{1}{\sqrt{2}} \\
0 \\
-\frac{1}{\sqrt{2}}
\end{array}\right)=0 \\
B_{3 g}=\left(\begin{array}{lll}
0 & 1 & 0
\end{array}\right) \cdot\left(\begin{array}{lll}
0 & 0 & 0 \\
0 & 0 & f \\
0 & f & 0
\end{array}\right) \cdot\left(\begin{array}{l}
0 \\
1 \\
0
\end{array}\right)=0 \\
B_{3 g}=\left(\begin{array}{lll}
0 & 1 & 0
\end{array}\right) \cdot\left(\begin{array}{lll}
0 & 0 & 0 \\
0 & 0 & f \\
0 & f & 0
\end{array}\right) \cdot\left(\begin{array}{l}
0 \\
1 \\
0
\end{array}\right)=0
\end{gathered}
$$

XY Konfiguration $B_{3 g}$ Mode:

$$
\begin{gathered}
B_{3 g}=\left(\begin{array}{lll}
\frac{1}{\sqrt{2}} & 0 & -\frac{1}{\sqrt{2}}
\end{array}\right) \cdot\left(\begin{array}{lll}
0 & 0 & 0 \\
0 & 0 & f \\
0 & f & 0
\end{array}\right) \cdot\left(\begin{array}{c}
\frac{1}{\sqrt{2}} \\
0 \\
\frac{1}{\sqrt{2}}
\end{array}\right)=\frac{1}{\sqrt{2}} \cdot f \\
B_{3 g}=\left(\begin{array}{lll}
-\frac{1}{\sqrt{2}} & 0 & -\frac{1}{\sqrt{2}}
\end{array}\right) \cdot\left(\begin{array}{lll}
0 & 0 & 0 \\
0 & 0 & f \\
0 & f & 0
\end{array}\right) \cdot\left(\begin{array}{c}
\frac{1}{\sqrt{2}} \\
0 \\
-\frac{1}{\sqrt{2}}
\end{array}\right)=\frac{1}{\sqrt{2}} \cdot f \\
B_{3 g}=\left(\begin{array}{lll}
\frac{1}{\sqrt{2}} & 0 & -\frac{1}{\sqrt{2}}
\end{array}\right) \cdot\left(\begin{array}{lll}
0 & 0 & 0 \\
0 & 0 & f \\
0 & f & 0
\end{array}\right) \cdot\left(\begin{array}{c}
0 \\
1 \\
0
\end{array}\right)=\frac{1}{\sqrt{2}} \cdot f \\
B_{3 g}=\left(\begin{array}{lll}
-\frac{1}{\sqrt{2}} & 0 & -\frac{1}{\sqrt{2}}
\end{array}\right) \cdot\left(\begin{array}{lll}
0 & 0 & 0 \\
0 & 0 & f \\
0 & f & 0
\end{array}\right) \cdot\left(\begin{array}{c}
0 \\
1 \\
0
\end{array}\right)=\frac{1}{\sqrt{2}} \cdot f \\
B_{3 g}=\left(\begin{array}{lll}
0 & 1 & 0
\end{array}\right) \cdot\left(\begin{array}{lll}
0 & 0 & 0 \\
0 & 0 & f \\
0 & f & 0
\end{array}\right) \cdot\left(\begin{array}{c}
-\frac{1}{\sqrt{2}} \\
0 \\
\frac{1}{\sqrt{2}}
\end{array}\right)=0 \\
B_{3 g}=\left(\begin{array}{lll}
0 & 1 & 0
\end{array}\right) \cdot\left(\begin{array}{lll}
0 & 0 & 0 \\
0 & 0 & f \\
0 & f & 0
\end{array}\right) \cdot\left(\begin{array}{c}
\frac{1}{\sqrt{2}} \\
0 \\
\frac{1}{\sqrt{2}}
\end{array}\right)=0
\end{gathered}
$$

X'X' Konfiguration $B_{3 g}$ Mode:

$$
B_{3 g}=\left(\begin{array}{lll}
1 & 0 & 0
\end{array}\right) \cdot\left(\begin{array}{ccc}
0 & 0 & 0 \\
0 & 0 & f \\
0 & f & 0
\end{array}\right) \cdot\left(\begin{array}{l}
1 \\
0 \\
0
\end{array}\right)=\frac{1}{\sqrt{2}} \cdot f
$$




$$
\begin{gathered}
B_{3 g}=\left(\begin{array}{lll}
0 & 0 & -1
\end{array}\right) \cdot\left(\begin{array}{lll}
0 & 0 & 0 \\
0 & 0 & f \\
0 & f & 0
\end{array}\right) \cdot\left(\begin{array}{c}
0 \\
0 \\
-1
\end{array}\right)=\frac{1}{\sqrt{2}} \cdot f \\
B_{3 g}=\left(\begin{array}{lll}
\frac{1}{2} & \frac{1}{\sqrt{2}} & -\frac{1}{2}
\end{array}\right) \cdot\left(\begin{array}{lll}
0 & 0 & 0 \\
0 & 0 & f \\
0 & f & 0
\end{array}\right) \cdot\left(\begin{array}{c}
\frac{1}{2} \\
\frac{1}{\sqrt{2}} \\
-\frac{1}{2}
\end{array}\right)=\frac{1}{\sqrt{2}} \cdot f \\
B_{3 g}=\left(\begin{array}{lll}
-\frac{1}{2} & \frac{1}{\sqrt{2}} & -\frac{1}{2}
\end{array}\right) \cdot\left(\begin{array}{lll}
0 & 0 & 0 \\
0 & 0 & f \\
0 & f & 0
\end{array}\right) \cdot\left(\begin{array}{c}
-\frac{1}{2} \\
\frac{1}{\sqrt{2}} \\
-\frac{1}{2}
\end{array}\right)=\frac{1}{\sqrt{2}} \cdot f \\
B_{3 g}=\left(\begin{array}{lll}
-\frac{1}{2} & \frac{1}{\sqrt{2}} & \frac{1}{2}
\end{array}\right) \cdot\left(\begin{array}{lll}
0 & 0 & 0 \\
0 & 0 & f \\
0 & f & 0
\end{array}\right) \cdot\left(\begin{array}{c}
-\frac{1}{2} \\
\frac{1}{\sqrt{2}} \\
\frac{1}{2}
\end{array}\right)=0 \\
B_{3 g}=\left(\begin{array}{lll}
\frac{1}{2} & \frac{1}{\sqrt{2}} & \frac{1}{2}
\end{array}\right) \cdot\left(\begin{array}{lll}
0 & 0 & 0 \\
0 & 0 & f \\
0 & f & 0
\end{array}\right) \cdot\left(\begin{array}{c}
\frac{1}{2} \\
\frac{1}{\sqrt{2}} \\
\frac{1}{2}
\end{array}\right)=0
\end{gathered}
$$

X'Y' Konfiguration $B_{3 g}$ Mode:

$$
\begin{gathered}
B_{3 g}=\left(\begin{array}{lll}
1 & 0 & 0
\end{array}\right) \cdot\left(\begin{array}{lll}
0 & 0 & 0 \\
0 & 0 & f \\
0 & f & 0
\end{array}\right) \cdot\left(\begin{array}{l}
0 \\
0 \\
1
\end{array}\right)=0 \\
B_{3 g}=\left(\begin{array}{lll}
0 & 0 & -1
\end{array}\right) \cdot\left(\begin{array}{lll}
0 & 0 & 0 \\
0 & 0 & f \\
0 & f & 0
\end{array}\right) \cdot\left(\begin{array}{l}
1 \\
0 \\
0
\end{array}\right)=0 \\
B_{3 g}=\left(\begin{array}{lll}
\frac{1}{2} & \frac{1}{\sqrt{2}} & -\frac{1}{2}
\end{array}\right) \cdot\left(\begin{array}{lll}
0 & 0 & 0 \\
0 & 0 & f \\
0 & f & 0
\end{array}\right) \cdot\left(\begin{array}{c}
-\frac{1}{2} \\
\frac{1}{\sqrt{2}} \\
\frac{1}{2}
\end{array}\right)=0 \\
B_{3 g}=\left(\begin{array}{lll}
-\frac{1}{2} & \frac{1}{\sqrt{2}} & -\frac{1}{2}
\end{array}\right) \cdot\left(\begin{array}{lll}
0 & 0 & 0 \\
0 & 0 & f \\
0 & f & 0
\end{array}\right) \cdot\left(\begin{array}{c}
\frac{1}{2} \\
\frac{1}{\sqrt{2}} \\
\frac{1}{2}
\end{array}\right)=0 \\
B_{3 g}=\left(\begin{array}{lll}
-\frac{1}{2} & \frac{1}{\sqrt{2}} & \frac{1}{2}
\end{array}\right) \cdot\left(\begin{array}{lll}
0 & 0 & 0 \\
0 & 0 & f \\
0 & f & 0
\end{array}\right) \cdot\left(\begin{array}{c}
-\frac{1}{2} \\
-\frac{1}{\sqrt{2}} \\
\frac{1}{2}
\end{array}\right)=0 \\
B_{3 g}=\left(\begin{array}{lll}
\frac{1}{2} & \frac{1}{\sqrt{2}} & \frac{1}{2}
\end{array}\right) \cdot\left(\begin{array}{lll}
0 & 0 & 0 \\
0 & 0 & f \\
0 & f & 0
\end{array}\right) \cdot\left(\begin{array}{c}
\frac{1}{2} \\
-\frac{1}{\sqrt{2}} \\
\frac{1}{2}
\end{array}\right)=0
\end{gathered}
$$


Alle diese Elemente müssen noch quadriert und mit dem Faktor 1/6 gewichtet werden. Das Ergebnis der mittleren Intensität ergibt sich dann zu:

\begin{tabular}{|c|c|c|c|c|}
\hline Mode & XX & XY & $X^{\prime} X^{\prime}$ & $X^{\prime} Y^{\prime}$ \\
\hline$A_{g}$ & $\frac{1}{6}\left[(a+c)^{2}+2 b^{2}\right]$ & $\frac{1}{12}(a-c)^{2}$ & $\frac{1}{6}\left[a^{2}+c^{2}+\frac{1}{4}(a+2 b+c)^{2}\right]$ & $\frac{1}{24}(a-2 b+c)^{2}$ \\
\hline$B_{1 g}$ & 0 & $\frac{2}{6} d^{2}$ & $\frac{2}{6} d^{2}$ & 0 \\
\hline$B_{2 g}$ & $\frac{4}{6} e^{2}$ & 0 & $\frac{1}{6} e^{2}$ & $\frac{3}{6} e^{2}$ \\
\hline$B_{3 g}$ & 0 & $\frac{2}{6} f^{2}$ & $\frac{2}{6} f^{2}$ & 0 \\
\hline
\end{tabular}

Damit ergeben sich folgende Intensitätsverhältnisse für die [001]-Richtung.

\begin{tabular}{|c|c|c|c|c|}
\hline Mode & XX & XY & X'X' $^{\prime}$ & $\mathrm{X}^{\prime} Y^{\prime}$ \\
\hline$B_{1 g}$ & 0 & 1 & 1 & 0 \\
\hline$B_{2 g}$ & 4 & 0 & 1 & 3 \\
\hline$B_{3 g}$ & 0 & 1 & 1 & 0 \\
\hline$A_{g}(a=b=c)$ & 6 & 0 & 6 & 0 \\
\hline$A_{g}(a=c \gg b)$ & 4 & 0 & 3 & 1 \\
\hline$A_{g}(a=c \ll b)$ & 2 & 0 & 1 & 1 \\
\hline
\end{tabular}

Tabelle 11 - Intensitätsverhältnisse für die Proben mit (001)Orientierung 
Für die (011) Ebene können sechs Zwillingsvarianten definiert werden. Deren Einheitsvektoren projiziert auf die Observationsebene lauten:

Zwilling 1:

$$
x=\left(\begin{array}{c}
\frac{1}{\sqrt{2}} \\
\frac{1}{2} \\
-\frac{1}{2}
\end{array}\right) y=\left(\begin{array}{c}
\frac{1}{\sqrt{2}} \\
-\frac{1}{2} \\
\frac{1}{2}
\end{array}\right) x^{\prime}=\left(\begin{array}{l}
1 \\
0 \\
0
\end{array}\right) y^{\prime}=\left(\begin{array}{c}
0 \\
-\frac{1}{\sqrt{2}} \\
\frac{1}{\sqrt{2}}
\end{array}\right)
$$

Zwilling 2:

$$
x=\left(\begin{array}{c}
-\frac{1}{\sqrt{2}} \\
\frac{1}{2} \\
-\frac{1}{2}
\end{array}\right) y=\left(\begin{array}{c}
\frac{1}{\sqrt{2}} \\
\frac{1}{2} \\
-\frac{1}{2}
\end{array}\right) x^{\prime}=\left(\begin{array}{c}
0 \\
\frac{1}{\sqrt{2}} \\
-\frac{1}{\sqrt{2}}
\end{array}\right) y^{\prime}=\left(\begin{array}{l}
1 \\
0 \\
0
\end{array}\right)
$$

Zwilling 3:

$$
x=\left(\begin{array}{c}
\frac{1}{\sqrt{2}} \\
\frac{1}{2} \\
-\frac{1}{2}
\end{array}\right) y=\left(\begin{array}{c}
0 \\
\frac{1}{\sqrt{2}} \\
\frac{1}{\sqrt{2}}
\end{array}\right) x^{\prime}=\left(\begin{array}{c}
\frac{1}{2} \\
\frac{2+\sqrt{2}}{4} \\
\frac{2-\sqrt{2}}{4}
\end{array}\right) y^{\prime}=\left(\begin{array}{c}
-\frac{1}{2} \\
\frac{2-\sqrt{2}}{4} \\
\frac{2+\sqrt{2}}{4}
\end{array}\right)
$$

Zwilling 4:

$$
x=\left(\begin{array}{c}
-\frac{1}{\sqrt{2}} \\
\frac{1}{2} \\
-\frac{1}{2}
\end{array}\right) y=\left(\begin{array}{c}
0 \\
\frac{1}{\sqrt{2}} \\
\frac{1}{\sqrt{2}}
\end{array}\right) x^{\prime}=\left(\begin{array}{c}
-\frac{1}{2} \\
\frac{2+\sqrt{2}}{4} \\
\frac{2-\sqrt{2}}{4}
\end{array}\right) y^{\prime}=\left(\begin{array}{c}
\frac{1}{2} \\
\frac{2-\sqrt{2}}{4} \\
\frac{2+\sqrt{2}}{4}
\end{array}\right)
$$

Zwilling 5:

$$
x=\left(\begin{array}{c}
0 \\
\frac{1}{\sqrt{2}} \\
\frac{1}{\sqrt{2}}
\end{array}\right) y=\left(\begin{array}{c}
-\frac{1}{\sqrt{2}} \\
-\frac{1}{2} \\
\frac{1}{2}
\end{array}\right) x^{\prime}=\left(\begin{array}{c}
-\frac{1}{2} \\
\frac{2-\sqrt{2}}{4} \\
\frac{2+\sqrt{2}}{4}
\end{array}\right) y^{\prime}=\left(\begin{array}{c}
-\frac{1}{2} \\
\frac{-2-\sqrt{2}}{4} \\
\frac{-2+\sqrt{2}}{4}
\end{array}\right)
$$

Zwilling 6:

$$
x=\left(\begin{array}{c}
0 \\
\frac{1}{\sqrt{2}} \\
\frac{1}{\sqrt{2}}
\end{array}\right) y=\left(\begin{array}{c}
\frac{1}{\sqrt{2}} \\
-\frac{1}{2} \\
\frac{1}{2}
\end{array}\right) x^{\prime}=\left(\begin{array}{c}
\frac{1}{2} \\
\frac{2-\sqrt{2}}{4} \\
\frac{2+\sqrt{2}}{4}
\end{array}\right) y^{\prime}=\left(\begin{array}{c}
\frac{1}{2} \\
\frac{-2-\sqrt{2}}{4} \\
\frac{-2+\sqrt{2}}{4}
\end{array}\right)
$$

Damit ergeben sich mit $I_{\text {Raman }}=A \cdot\left[\sum e_{i}^{\sigma} R_{\rho \sigma} e_{s}^{\rho}\right]^{2}$ folgende Intensitäten: XX Konfiguration $A_{g}$ Mode:

$$
A_{g}=\left(\begin{array}{lll}
\frac{1}{\sqrt{2}} & \frac{1}{2} & -\frac{1}{2}
\end{array}\right) \cdot\left(\begin{array}{lll}
a & 0 & 0 \\
0 & b & 0 \\
0 & 0 & c
\end{array}\right) \cdot\left(\begin{array}{c}
\frac{1}{\sqrt{2}} \\
\frac{1}{2} \\
-\frac{1}{2}
\end{array}\right)=\frac{1}{4}(2 a+b+c)
$$




$$
\begin{gathered}
A_{g}=\left(\begin{array}{lll}
-\frac{1}{\sqrt{2}} & \frac{1}{2} & -\frac{1}{2}
\end{array}\right) \cdot\left(\begin{array}{lll}
a & 0 & 0 \\
0 & b & 0 \\
0 & 0 & c
\end{array}\right) \cdot\left(\begin{array}{c}
-\frac{1}{\sqrt{2}} \\
\frac{1}{2} \\
-\frac{1}{2}
\end{array}\right)=\frac{1}{4}(2 a+b+c) \\
A_{g}=\left(\begin{array}{lll}
\frac{1}{\sqrt{2}} & \frac{1}{2} & -\frac{1}{2}
\end{array}\right) \cdot\left(\begin{array}{lll}
a & 0 & 0 \\
0 & b & 0 \\
0 & 0 & c
\end{array}\right) \cdot\left(\begin{array}{c}
\frac{1}{\sqrt{2}} \\
\frac{1}{2} \\
-\frac{1}{2}
\end{array}\right)=\frac{1}{4}(2 a+b+c) \\
A_{g}=\left(\begin{array}{lll}
-\frac{1}{\sqrt{2}} & \frac{1}{2} & -\frac{1}{2}
\end{array}\right) \cdot\left(\begin{array}{lll}
a & 0 & 0 \\
0 & b & 0 \\
0 & 0 & c
\end{array}\right) \cdot\left(\begin{array}{c}
-\frac{1}{\sqrt{2}} \\
\frac{1}{2} \\
-\frac{1}{2}
\end{array}\right)=\frac{1}{4}(2 a+b+c) \\
A_{g}=\left(\begin{array}{lll}
0 & \frac{1}{\sqrt{2}} & \frac{1}{\sqrt{2}}
\end{array}\right) \cdot\left(\begin{array}{lll}
a & 0 & 0 \\
0 & b & 0 \\
0 & 0 & c
\end{array}\right) \cdot\left(\begin{array}{c}
0 \\
\frac{1}{\sqrt{2}} \\
\frac{1}{\sqrt{2}}
\end{array}\right)=\frac{1}{2}(b+c) \\
A_{g}=\left(\begin{array}{lll}
0 & \frac{1}{\sqrt{2}} & \frac{1}{\sqrt{2}}
\end{array}\right) \cdot\left(\begin{array}{lll}
a & 0 & 0 \\
0 & b & 0 \\
0 & 0 & c
\end{array}\right) \cdot\left(\begin{array}{c}
0 \\
\frac{1}{\sqrt{2}} \\
\frac{1}{\sqrt{2}}
\end{array}\right)=\frac{1}{2}(b+c)
\end{gathered}
$$

XY Konfiguration $A_{g}$ Mode:

$$
\begin{aligned}
A_{g}=\left(\begin{array}{lll}
\frac{1}{\sqrt{2}} & \frac{1}{2} & -\frac{1}{2}
\end{array}\right) \cdot\left(\begin{array}{lll}
a & 0 & 0 \\
0 & b & 0 \\
0 & 0 & c
\end{array}\right) \cdot\left(\begin{array}{c}
\frac{1}{\sqrt{2}} \\
-\frac{1}{2} \\
\frac{1}{2}
\end{array}\right)=\frac{1}{4}(2 a-b-c) \\
A_{g}=\left(\begin{array}{lll}
-\frac{1}{\sqrt{2}} & \frac{1}{2} & -\frac{1}{2}
\end{array}\right) \cdot\left(\begin{array}{lll}
a & 0 & 0 \\
0 & b & 0 \\
0 & 0 & c
\end{array}\right) \cdot\left(\begin{array}{c}
\frac{1}{\sqrt{2}} \\
\frac{1}{2} \\
-\frac{1}{2}
\end{array}\right)=\frac{1}{4}(2 a-b-c) \\
A_{g}=\left(\begin{array}{lll}
\frac{1}{\sqrt{2}} & \frac{1}{2} & -\frac{1}{2}
\end{array}\right) \cdot\left(\begin{array}{lll}
a & 0 & 0 \\
0 & b & 0 \\
0 & 0 & c
\end{array}\right) \cdot\left(\begin{array}{c}
0 \\
\frac{1}{\sqrt{2}} \\
\frac{1}{\sqrt{2}}
\end{array}\right)=\frac{1}{2 \sqrt{2}}(b-c) \\
A_{g}=\left(\begin{array}{lll}
-\frac{1}{\sqrt{2}} & \frac{1}{2} & -\frac{1}{2}
\end{array}\right) \cdot\left(\begin{array}{lll}
a & 0 & 0 \\
0 & b & 0 \\
0 & 0 & c
\end{array}\right) \cdot\left(\begin{array}{c}
0 \\
\frac{1}{\sqrt{2}} \\
\frac{1}{\sqrt{2}}
\end{array}\right)=\frac{1}{2 \sqrt{2}}(b-c) \\
A_{g}=\left(\begin{array}{lll}
0 & \frac{1}{\sqrt{2}} & \frac{1}{\sqrt{2}}
\end{array}\right) \cdot\left(\begin{array}{lll}
a & 0 & 0 \\
0 & b & 0 \\
0 & 0 & c
\end{array}\right) \cdot\left(\begin{array}{c}
-\frac{1}{\sqrt{2}} \\
-\frac{1}{2} \\
\frac{1}{2}
\end{array}\right)=\frac{1}{2 \sqrt{2}}(b-c) \\
A_{g}=\left(\begin{array}{lll}
0 & \frac{1}{\sqrt{2}} & \frac{1}{\sqrt{2}}
\end{array}\right) \cdot\left(\begin{array}{lll}
a & 0 & 0 \\
0 & b & 0 \\
0 & 0 & c
\end{array}\right) \cdot\left(\begin{array}{c}
\frac{1}{\sqrt{2}} \\
-\frac{1}{2} \\
\frac{1}{2}
\end{array}\right)=\frac{1}{2 \sqrt{2}}(b-c)
\end{aligned}
$$


X'X' Konfiguration $A_{g}$ Mode:

$$
\begin{gathered}
A_{g}=\left(\begin{array}{lll}
1 & 0 & 0
\end{array}\right) \cdot\left(\begin{array}{lll}
a & 0 & 0 \\
0 & b & 0 \\
0 & 0 & c
\end{array}\right) \cdot\left(\begin{array}{l}
1 \\
0 \\
0
\end{array}\right)=a \\
A_{g}=\left(\begin{array}{lll}
0 & \frac{1}{\sqrt{2}} & -\frac{1}{\sqrt{2}}
\end{array}\right) \cdot\left(\begin{array}{lll}
a & 0 & 0 \\
0 & b & 0 \\
0 & 0 & c
\end{array}\right) \cdot\left(\begin{array}{c}
0 \\
\frac{1}{\sqrt{2}} \\
-\frac{1}{\sqrt{2}}
\end{array}\right)=\frac{1}{2}(b+c) \\
A_{g}=\left(\begin{array}{lll}
\frac{1}{2} & \frac{2+\sqrt{2}}{4} & \frac{2-\sqrt{2}}{4}
\end{array}\right) \cdot\left(\begin{array}{lll}
a & 0 & 0 \\
0 & b & 0 \\
0 & 0 & c
\end{array}\right) \cdot\left(\begin{array}{c}
\frac{1}{2} \\
\frac{2+\sqrt{2}}{4} \\
\frac{2-\sqrt{2}}{4}
\end{array}\right)=\frac{1}{8}(2 a+(3+\sqrt{2}) b+(3-\sqrt{2}) c) \\
A_{g}=\left(\begin{array}{lll}
-\frac{1}{2} & \frac{2+\sqrt{2}}{4} & \frac{2-\sqrt{2}}{4}
\end{array}\right) \cdot\left(\begin{array}{lll}
a & 0 & 0 \\
0 & b & 0 \\
0 & 0 & c
\end{array}\right) \cdot\left(\begin{array}{c}
-\frac{1}{2} \\
\frac{2+\sqrt{2}}{4} \\
\frac{2-\sqrt{2}}{4}
\end{array}\right)=\frac{1}{8}(2 a+(3+\sqrt{2}) b+(3-\sqrt{2}) c) \\
A_{g}=\left(\begin{array}{lll}
-\frac{1}{2} & \frac{2-\sqrt{2}}{4} & \frac{2+\sqrt{2}}{4}
\end{array}\right) \cdot\left(\begin{array}{lll}
a & 0 & 0 \\
0 & b & 0 \\
0 & 0 & c
\end{array}\right) \cdot\left(\begin{array}{c}
-\frac{1}{2} \\
\frac{2-\sqrt{2}}{4} \\
\frac{2+\sqrt{2}}{4}
\end{array}\right)=\frac{1}{8}(2 a-(3+\sqrt{2}) b+(3+\sqrt{2}) c) \\
A_{g}=\left(\begin{array}{llll}
\frac{1}{2} & \frac{2-\sqrt{2}}{4} & \frac{2+\sqrt{2}}{4}
\end{array}\right) \cdot\left(\begin{array}{lll}
a & 0 & 0 \\
0 & b & 0 \\
0 & 0 & c
\end{array}\right) \cdot\left(\begin{array}{c}
\frac{1}{2} \\
\frac{2-\sqrt{2}}{4} \\
\frac{2+\sqrt{2}}{4}
\end{array}\right)=\frac{1}{8}(2 a+(3-\sqrt{2}) b+(3+\sqrt{2}) c)
\end{gathered}
$$

X'Y' Konfiguration $A_{g}$ Mode:

$$
\begin{gathered}
A_{g}=\left(\begin{array}{lll}
1 & 0 & 0
\end{array}\right) \cdot\left(\begin{array}{lll}
a & 0 & 0 \\
0 & b & 0 \\
0 & 0 & c
\end{array}\right) \cdot\left(\begin{array}{c}
0 \\
-\frac{1}{\sqrt{2}} \\
\frac{1}{\sqrt{2}}
\end{array}\right)=0 \\
A_{g}=\left(\begin{array}{lll}
0 & \frac{1}{\sqrt{2}} & -\frac{1}{\sqrt{2}}
\end{array}\right) \cdot\left(\begin{array}{lll}
a & 0 & 0 \\
0 & b & 0 \\
0 & 0 & c
\end{array}\right) \cdot\left(\begin{array}{l}
1 \\
0 \\
0
\end{array}\right)=0 \\
A_{g}=\left(\begin{array}{lll}
\frac{1}{2} & \frac{2+\sqrt{2}}{4} & \frac{2-\sqrt{2}}{4}
\end{array}\right) \cdot\left(\begin{array}{lll}
a & 0 & 0 \\
0 & b & 0 \\
0 & 0 & c
\end{array}\right) \cdot\left(\begin{array}{c}
-\frac{1}{2} \\
\frac{2-\sqrt{2}}{4} \\
\frac{2+\sqrt{2}}{4}
\end{array}\right)=\frac{1}{8}(2 a-b-c) \\
A_{g}=\left(\begin{array}{llll}
-\frac{1}{2} & \frac{2+\sqrt{2}}{4} & \frac{2-\sqrt{2}}{4}
\end{array}\right) \cdot\left(\begin{array}{lll}
a & 0 & 0 \\
0 & b & 0 \\
0 & 0 & c
\end{array}\right) \cdot\left(\begin{array}{c}
\frac{1}{2} \\
\frac{2-\sqrt{2}}{4} \\
\frac{2+\sqrt{2}}{4}
\end{array}\right)=\frac{1}{8}(2 a-b-c)
\end{gathered}
$$




$$
\begin{gathered}
A_{g}=\left(\begin{array}{lll}
-\frac{1}{2} & \frac{2-\sqrt{2}}{4} & \frac{2+\sqrt{2}}{4}
\end{array}\right) \cdot\left(\begin{array}{lll}
a & 0 & 0 \\
0 & b & 0 \\
0 & 0 & c
\end{array}\right) \cdot\left(\begin{array}{c}
\frac{-1}{2} \\
\frac{-2-\sqrt{2}}{4} \\
\frac{-2+\sqrt{2}}{4}
\end{array}\right)=\frac{1}{8}(2 a-b-c) \\
A_{g}=\left(\begin{array}{lll}
\frac{1}{2} & \frac{2-\sqrt{2}}{4} & \frac{2+\sqrt{2}}{4}
\end{array}\right) \cdot\left(\begin{array}{lll}
a & 0 & 0 \\
0 & b & 0 \\
0 & 0 & c
\end{array}\right) \cdot\left(\begin{array}{c}
\frac{1}{2} \\
\frac{-2-\sqrt{2}}{4} \\
\frac{-2+\sqrt{2}}{4}
\end{array}\right)=\frac{1}{8}(2 a-b-c)
\end{gathered}
$$

XX Konfiguration $B_{1 g}$ Mode:

$$
\begin{gathered}
B_{1 g}=\left(\begin{array}{lll}
\frac{1}{\sqrt{2}} & \frac{1}{2} & -\frac{1}{2}
\end{array}\right) \cdot\left(\begin{array}{lll}
0 & d & 0 \\
d & 0 & 0 \\
0 & 0 & 0
\end{array}\right) \cdot\left(\begin{array}{c}
\frac{1}{\sqrt{2}} \\
\frac{1}{2} \\
-\frac{1}{2}
\end{array}\right)=\frac{1}{\sqrt{2}} d \\
B_{1 g}=\left(\begin{array}{lll}
-\frac{1}{\sqrt{2}} & \frac{1}{2} & -\frac{1}{2}
\end{array}\right) \cdot\left(\begin{array}{lll}
0 & d & 0 \\
d & 0 & 0 \\
0 & 0 & 0
\end{array}\right) \cdot\left(\begin{array}{c}
-\frac{1}{\sqrt{2}} \\
\frac{1}{2} \\
-\frac{1}{2}
\end{array}\right)=\frac{1}{\sqrt{2}} d \\
B_{1 g}=\left(\begin{array}{lll}
\frac{1}{\sqrt{2}} & \frac{1}{2} & -\frac{1}{2}
\end{array}\right) \cdot\left(\begin{array}{lll}
0 & d & 0 \\
d & 0 & 0 \\
0 & 0 & 0
\end{array}\right) \cdot\left(\begin{array}{c}
\frac{1}{\sqrt{2}} \\
\frac{1}{2} \\
-\frac{1}{2}
\end{array}\right)=\frac{1}{\sqrt{2}} d \\
B_{1 g}=\left(\begin{array}{lll}
-\frac{1}{\sqrt{2}} & \frac{1}{2} & -\frac{1}{2}
\end{array}\right) \cdot\left(\begin{array}{lll}
0 & d & 0 \\
d & 0 & 0 \\
0 & 0 & 0
\end{array}\right) \cdot\left(\begin{array}{c}
-\frac{1}{\sqrt{2}} \\
\frac{1}{2} \\
-\frac{1}{2}
\end{array}\right)=\frac{1}{\sqrt{2}} d \\
B_{1 g}=\left(\begin{array}{lll}
0 & \frac{1}{\sqrt{2}} & \frac{1}{\sqrt{2}}
\end{array}\right) \cdot\left(\begin{array}{lll}
0 & d & 0 \\
d & 0 & 0 \\
0 & 0 & 0
\end{array}\right) \cdot\left(\begin{array}{c}
0 \\
\frac{1}{\sqrt{2}} \\
\frac{1}{\sqrt{2}}
\end{array}\right)=0 \\
B_{1 g}=\left(\begin{array}{lll}
0 & \frac{1}{\sqrt{2}} & \frac{1}{\sqrt{2}}
\end{array}\right) \cdot\left(\begin{array}{lll}
0 & d & 0 \\
d & 0 & 0 \\
0 & 0 & 0
\end{array}\right) \cdot\left(\begin{array}{c}
0 \\
\frac{1}{\sqrt{2}} \\
\frac{1}{\sqrt{2}}
\end{array}\right)=0
\end{gathered}
$$

XY Konfiguration $B_{1 g}$ Mode:

$$
\begin{gathered}
B_{1 g}=\left(\begin{array}{lll}
\frac{1}{\sqrt{2}} & \frac{1}{2} & -\frac{1}{2}
\end{array}\right) \cdot\left(\begin{array}{lll}
0 & d & 0 \\
d & 0 & 0 \\
0 & 0 & 0
\end{array}\right) \cdot\left(\begin{array}{c}
\frac{1}{\sqrt{2}} \\
-\frac{1}{2} \\
\frac{1}{2}
\end{array}\right)=0 \\
B_{1 g}=\left(\begin{array}{lll}
-\frac{1}{\sqrt{2}} & \frac{1}{2} & -\frac{1}{2}
\end{array}\right) \cdot\left(\begin{array}{lll}
0 & d & 0 \\
d & 0 & 0 \\
0 & 0 & 0
\end{array}\right) \cdot\left(\begin{array}{c}
\frac{1}{\sqrt{2}} \\
\frac{1}{2} \\
-\frac{1}{2}
\end{array}\right)=0
\end{gathered}
$$




$$
\begin{aligned}
& B_{1 g}=\left(\begin{array}{lll}
\frac{1}{\sqrt{2}} & \frac{1}{2} & -\frac{1}{2}
\end{array}\right) \cdot\left(\begin{array}{lll}
0 & d & 0 \\
d & 0 & 0 \\
0 & 0 & 0
\end{array}\right) \cdot\left(\begin{array}{c}
0 \\
\frac{1}{\sqrt{2}} \\
\frac{1}{\sqrt{2}}
\end{array}\right)=\frac{1}{2} d \\
& B_{1 g}=\left(\begin{array}{lll}
-\frac{1}{\sqrt{2}} & \frac{1}{2} & -\frac{1}{2}
\end{array}\right) \cdot\left(\begin{array}{lll}
0 & d & 0 \\
d & 0 & 0 \\
0 & 0 & 0
\end{array}\right) \cdot\left(\begin{array}{c}
0 \\
\frac{1}{\sqrt{2}} \\
\frac{1}{\sqrt{2}}
\end{array}\right)=\frac{1}{2} d \\
& B_{1 g}=\left(\begin{array}{lll}
0 & \frac{1}{\sqrt{2}} & \frac{1}{\sqrt{2}}
\end{array}\right) \cdot\left(\begin{array}{lll}
0 & d & 0 \\
d & 0 & 0 \\
0 & 0 & 0
\end{array}\right) \cdot\left(\begin{array}{c}
-\frac{1}{\sqrt{2}} \\
-\frac{1}{2} \\
\frac{1}{2}
\end{array}\right)=\frac{1}{2} d \\
& B_{1 g}=\left(\begin{array}{lll}
0 & \frac{1}{\sqrt{2}} & \frac{1}{\sqrt{2}}
\end{array}\right) \cdot\left(\begin{array}{lll}
0 & d & 0 \\
d & 0 & 0 \\
0 & 0 & 0
\end{array}\right) \cdot\left(\begin{array}{c}
\frac{1}{\sqrt{2}} \\
-\frac{1}{2} \\
\frac{1}{2}
\end{array}\right)=\frac{1}{2} d
\end{aligned}
$$

X'X' Konfiguration $B_{1 g}$ Mode:

$$
\begin{gathered}
B_{1 g}=\left(\begin{array}{lll}
1 & 0 & 0
\end{array}\right) \cdot\left(\begin{array}{lll}
0 & d & 0 \\
d & 0 & 0 \\
0 & 0 & 0
\end{array}\right) \cdot\left(\begin{array}{l}
1 \\
0 \\
0
\end{array}\right)=0 \\
B_{1 g}=\left(\begin{array}{lll}
0 & \frac{1}{\sqrt{2}} & -\frac{1}{\sqrt{2}}
\end{array}\right) \cdot\left(\begin{array}{lll}
0 & d & 0 \\
d & 0 & 0 \\
0 & 0 & 0
\end{array}\right) \cdot\left(\begin{array}{c}
0 \\
\frac{1}{\sqrt{2}} \\
-\frac{1}{\sqrt{2}}
\end{array}\right)=0 \\
B_{1 g}=\left(\begin{array}{lll}
\frac{1}{2} & \frac{2+\sqrt{2}}{4} & \frac{2-\sqrt{2}}{4}
\end{array}\right) \cdot\left(\begin{array}{lll}
0 & d & 0 \\
d & 0 & 0 \\
0 & 0 & 0
\end{array}\right) \cdot\left(\begin{array}{c}
\frac{1}{2} \\
\frac{2+\sqrt{2}}{4} \\
\frac{2-\sqrt{2}}{4}
\end{array}\right)=\frac{2+\sqrt{2}}{4} d \\
B_{1 g}=\left(\begin{array}{lll}
-\frac{1}{2} & \frac{2+\sqrt{2}}{4} & \frac{2-\sqrt{2}}{4}
\end{array}\right) \cdot\left(\begin{array}{lll}
0 & d & 0 \\
d & 0 & 0 \\
0 & 0 & 0
\end{array}\right) \cdot\left(\begin{array}{c}
-\frac{1}{2} \\
\frac{2+\sqrt{2}}{4} \\
\frac{2-\sqrt{2}}{4}
\end{array}\right)=\frac{2+\sqrt{2}}{4} d \\
B_{1 g}=\left(\begin{array}{llll}
-\frac{1}{2} & \frac{2-\sqrt{2}}{4} & \frac{2+\sqrt{2}}{4}
\end{array}\right) \cdot\left(\begin{array}{lll}
0 & d & 0 \\
d & 0 & 0 \\
0 & 0 & 0
\end{array}\right) \cdot\left(\begin{array}{c}
-\frac{1}{2} \\
\frac{2-\sqrt{2}}{4} \\
\frac{2+\sqrt{2}}{4}
\end{array}\right)=\frac{-2+\sqrt{2}}{4} d \\
B_{1 g}=\left(\begin{array}{llll}
\frac{1}{2} & \frac{2-\sqrt{2}}{4} & \frac{2+\sqrt{2}}{4}
\end{array}\right) \cdot\left(\begin{array}{lll}
0 & d & 0 \\
d & 0 & 0 \\
0 & 0 & 0
\end{array}\right) \cdot\left(\begin{array}{c}
\frac{1}{2} \\
\frac{2-\sqrt{2}}{4} \\
\frac{2+\sqrt{2}}{4}
\end{array}\right)=\frac{-2+\sqrt{2}}{4} d
\end{gathered}
$$


X'Y' Konfiguration $B_{1 g}$ Mode:

$$
\begin{gathered}
B_{1 g}=\left(\begin{array}{lll}
1 & 0 & 0
\end{array}\right) \cdot\left(\begin{array}{lll}
0 & d & 0 \\
d & 0 & 0 \\
0 & 0 & 0
\end{array}\right) \cdot\left(\begin{array}{c}
0 \\
-\frac{1}{\sqrt{2}} \\
\frac{1}{\sqrt{2}}
\end{array}\right)=\frac{1}{\sqrt{2}} d \\
B_{1 g}=\left(\begin{array}{lll}
0 & \frac{1}{\sqrt{2}} & -\frac{1}{\sqrt{2}}
\end{array}\right) \cdot\left(\begin{array}{lll}
0 & d & 0 \\
d & 0 & 0 \\
0 & 0 & 0
\end{array}\right) \cdot\left(\begin{array}{l}
1 \\
0 \\
0
\end{array}\right)=\frac{1}{\sqrt{2}} d \\
B_{1 g}=\left(\begin{array}{lll}
\frac{1}{2} & \frac{2+\sqrt{2}}{4} & \frac{2-\sqrt{2}}{4}
\end{array}\right) \cdot\left(\begin{array}{lll}
0 & d & 0 \\
d & 0 & 0 \\
0 & 0 & 0
\end{array}\right) \cdot\left(\begin{array}{l}
-\frac{1}{2} \\
\frac{2-\sqrt{2}}{4} \\
\frac{2+\sqrt{2}}{4}
\end{array}\right)=\frac{\sqrt{2}}{4} d \\
B_{1 g}=\left(\begin{array}{lll}
-\frac{1}{2} & \frac{2+\sqrt{2}}{4} & \frac{2-\sqrt{2}}{4}
\end{array}\right) \cdot\left(\begin{array}{lll}
0 & d & 0 \\
d & 0 & 0 \\
0 & 0 & 0
\end{array}\right) \cdot\left(\begin{array}{c}
\frac{1}{2} \\
\frac{2-\sqrt{2}}{4} \\
\frac{2+\sqrt{2}}{4}
\end{array}\right)=\frac{\sqrt{2}}{4} d \\
B_{1 g}=\left(\begin{array}{lll}
-\frac{1}{2} & \frac{2-\sqrt{2}}{4} & \frac{2+\sqrt{2}}{4}
\end{array}\right) \cdot\left(\begin{array}{lll}
0 & d & 0 \\
d & 0 & 0 \\
0 & 0 & 0
\end{array}\right) \cdot\left(\begin{array}{c}
\frac{-1}{2} \\
\frac{-2-\sqrt{2}}{4} \\
\frac{-2+\sqrt{2}}{4}
\end{array}\right)=\frac{\sqrt{2}}{4} d \\
B_{1 g}=\left(\begin{array}{llll}
\frac{1}{2} & \frac{2-\sqrt{2}}{4} & \frac{2+\sqrt{2}}{4}
\end{array}\right) \cdot\left(\begin{array}{lll}
0 & d & 0 \\
d & 0 & 0 \\
0 & 0 & 0
\end{array}\right) \cdot\left(\begin{array}{c}
\frac{1}{2} \\
\frac{-2-\sqrt{2}}{4} \\
\frac{-2+\sqrt{2}}{4}
\end{array}\right)=\frac{\sqrt{2}}{4} d
\end{gathered}
$$

XX Konfiguration $B_{2 g}$ Mode:

$$
\begin{gathered}
B_{2 g}=\left(\begin{array}{lll}
\frac{1}{\sqrt{2}} & \frac{1}{2} & -\frac{1}{2}
\end{array}\right) \cdot\left(\begin{array}{lll}
0 & 0 & e \\
0 & 0 & 0 \\
e & 0 & 0
\end{array}\right) \cdot\left(\begin{array}{c}
\frac{1}{\sqrt{2}} \\
\frac{1}{2} \\
-\frac{1}{2}
\end{array}\right)=\frac{1}{\sqrt{2}} e \\
B_{2 g}=\left(\begin{array}{lll}
-\frac{1}{\sqrt{2}} & \frac{1}{2} & -\frac{1}{2}
\end{array}\right) \cdot\left(\begin{array}{lll}
0 & 0 & e \\
0 & 0 & 0 \\
e & 0 & 0
\end{array}\right) \cdot\left(\begin{array}{c}
-\frac{1}{\sqrt{2}} \\
\frac{1}{2} \\
-\frac{1}{2}
\end{array}\right)=\frac{1}{\sqrt{2}} e \\
B_{2 g}=\left(\begin{array}{lll}
\frac{1}{\sqrt{2}} & \frac{1}{2} & -\frac{1}{2}
\end{array}\right) \cdot\left(\begin{array}{lll}
0 & 0 & e \\
0 & 0 & 0 \\
e & 0 & 0
\end{array}\right) \cdot\left(\begin{array}{c}
\frac{1}{\sqrt{2}} \\
\frac{1}{2} \\
-\frac{1}{2}
\end{array}\right)=\frac{1}{\sqrt{2}} e \\
B_{2 g}=\left(\begin{array}{lll}
-\frac{1}{\sqrt{2}} & \frac{1}{2} & -\frac{1}{2}
\end{array}\right) \cdot\left(\begin{array}{lll}
0 & 0 & e \\
0 & 0 & 0 \\
e & 0 & 0
\end{array}\right) \cdot\left(\begin{array}{c}
-\frac{1}{\sqrt{2}} \\
\frac{1}{2} \\
-\frac{1}{2}
\end{array}\right)=\frac{1}{\sqrt{2}} e
\end{gathered}
$$




$$
\begin{aligned}
& B_{2 g}=\left(\begin{array}{lll}
0 & \frac{1}{\sqrt{2}} & \frac{1}{\sqrt{2}}
\end{array}\right) \cdot\left(\begin{array}{ccc}
0 & 0 & e \\
0 & 0 & 0 \\
e & 0 & 0
\end{array}\right) \cdot\left(\begin{array}{c}
0 \\
\frac{1}{\sqrt{2}} \\
\frac{1}{\sqrt{2}}
\end{array}\right)=0 \\
& B_{2 g}=\left(\begin{array}{lll}
0 & \frac{1}{\sqrt{2}} & \frac{1}{\sqrt{2}}
\end{array}\right) \cdot\left(\begin{array}{lll}
0 & 0 & e \\
0 & 0 & 0 \\
e & 0 & 0
\end{array}\right) \cdot\left(\begin{array}{c}
0 \\
\frac{1}{\sqrt{2}} \\
\frac{1}{\sqrt{2}}
\end{array}\right)=0
\end{aligned}
$$

XY Konfiguration $B_{2 g}$ Mode:

$$
\begin{gathered}
B_{2 g}=\left(\begin{array}{lll}
\frac{1}{\sqrt{2}} & \frac{1}{2} & -\frac{1}{2}
\end{array}\right) \cdot\left(\begin{array}{lll}
0 & 0 & e \\
0 & 0 & 0 \\
e & 0 & 0
\end{array}\right) \cdot\left(\begin{array}{c}
\frac{1}{\sqrt{2}} \\
-\frac{1}{2} \\
\frac{1}{2}
\end{array}\right)=0 \\
B_{2 g}=\left(\begin{array}{lll}
-\frac{1}{\sqrt{2}} & \frac{1}{2} & -\frac{1}{2}
\end{array}\right) \cdot\left(\begin{array}{lll}
0 & 0 & e \\
0 & 0 & 0 \\
e & 0 & 0
\end{array}\right) \cdot\left(\begin{array}{c}
\frac{1}{\sqrt{2}} \\
\frac{1}{2} \\
-\frac{1}{2}
\end{array}\right)=0 \\
B_{2 g}=\left(\begin{array}{lll}
\frac{1}{\sqrt{2}} & \frac{1}{2} & -\frac{1}{2}
\end{array}\right) \cdot\left(\begin{array}{lll}
0 & 0 & e \\
0 & 0 & 0 \\
e & 0 & 0
\end{array}\right) \cdot\left(\begin{array}{c}
0 \\
\frac{1}{\sqrt{2}} \\
\frac{1}{\sqrt{2}}
\end{array}\right)=\frac{1}{2} e \\
B_{2 g}=\left(\begin{array}{lll}
-\frac{1}{\sqrt{2}} & \frac{1}{2} & -\frac{1}{2}
\end{array}\right) \cdot\left(\begin{array}{lll}
0 & 0 & e \\
0 & 0 & 0 \\
e & 0 & 0
\end{array}\right) \cdot\left(\begin{array}{c}
0 \\
\frac{1}{\sqrt{2}} \\
\frac{1}{\sqrt{2}}
\end{array}\right)=-\frac{1}{2} e \\
B_{2 g}=\left(\begin{array}{lll}
0 & \frac{1}{\sqrt{2}} & \frac{1}{\sqrt{2}}
\end{array}\right) \cdot\left(\begin{array}{lll}
0 & 0 & e \\
0 & 0 & 0 \\
e & 0 & 0
\end{array}\right) \cdot\left(\begin{array}{c}
-\frac{1}{\sqrt{2}} \\
-\frac{1}{2} \\
\frac{1}{2}
\end{array}\right)=-\frac{1}{2} e \\
B_{2 g}=\left(\begin{array}{lll}
0 & \frac{1}{\sqrt{2}} & \frac{1}{\sqrt{2}}
\end{array}\right) \cdot\left(\begin{array}{lll}
0 & 0 & e \\
0 & 0 & 0 \\
e & 0 & 0
\end{array}\right) \cdot\left(\begin{array}{c}
\frac{1}{\sqrt{2}} \\
-\frac{1}{2} \\
\frac{1}{2}
\end{array}\right)=\frac{1}{2} e
\end{gathered}
$$

X'X' Konfiguration $B_{2 g}$ Mode:

$$
\begin{gathered}
B_{2 g}=\left(\begin{array}{lll}
1 & 0 & 0
\end{array}\right) \cdot\left(\begin{array}{lll}
0 & 0 & e \\
0 & 0 & 0 \\
e & 0 & 0
\end{array}\right) \cdot\left(\begin{array}{l}
1 \\
0 \\
0
\end{array}\right)=0 \\
B_{2 g}=\left(\begin{array}{lll}
0 & \frac{1}{\sqrt{2}} & -\frac{1}{\sqrt{2}}
\end{array}\right) \cdot\left(\begin{array}{lll}
0 & 0 & e \\
0 & 0 & 0 \\
e & 0 & 0
\end{array}\right) \cdot\left(\begin{array}{c}
0 \\
\frac{1}{\sqrt{2}} \\
-\frac{1}{\sqrt{2}}
\end{array}\right)=0
\end{gathered}
$$




$$
\begin{gathered}
B_{2 g}=\left(\begin{array}{lll}
\frac{1}{2} & \frac{2+\sqrt{2}}{4} & \frac{2-\sqrt{2}}{4}
\end{array}\right) \cdot\left(\begin{array}{lll}
0 & 0 & e \\
0 & 0 & 0 \\
e & 0 & 0
\end{array}\right) \cdot\left(\begin{array}{c}
\frac{1}{2} \\
\frac{2+\sqrt{2}}{4} \\
\frac{2-\sqrt{2}}{4}
\end{array}\right)=\frac{2-\sqrt{2}}{4} e \\
B_{2 g}=\left(\begin{array}{lll}
-\frac{1}{2} & \frac{2+\sqrt{2}}{4} & \frac{2-\sqrt{2}}{4}
\end{array}\right) \cdot\left(\begin{array}{lll}
0 & 0 & e \\
0 & 0 & 0 \\
e & 0 & 0
\end{array}\right) \cdot\left(\begin{array}{c}
-\frac{1}{2} \\
\frac{2+\sqrt{2}}{4} \\
\frac{2-\sqrt{2}}{4}
\end{array}\right)=\frac{2-\sqrt{2}}{4} e \\
B_{2 g}=\left(\begin{array}{lll}
-\frac{1}{2} & \frac{2-\sqrt{2}}{4} & \frac{2+\sqrt{2}}{4}
\end{array}\right) \cdot\left(\begin{array}{lll}
0 & 0 & e \\
0 & 0 & 0 \\
e & 0 & 0
\end{array}\right) \cdot\left(\begin{array}{l}
\frac{2-\frac{1}{2}}{4} \\
\frac{2+\sqrt{2}}{4}
\end{array}\right)=\frac{2+\sqrt{2}}{4} e \\
B_{2 g}=\left(\begin{array}{llll}
\frac{1}{2} & \frac{2-\sqrt{2}}{4} & \frac{2+\sqrt{2}}{4}
\end{array}\right) \cdot\left(\begin{array}{lll}
0 & 0 & e \\
0 & 0 & 0 \\
e & 0 & 0
\end{array}\right) \cdot\left(\begin{array}{c}
\frac{1}{2} \\
\frac{2-\sqrt{2}}{4} \\
\frac{2+\sqrt{2}}{4}
\end{array}\right)=\frac{2+\sqrt{2}}{4} e
\end{gathered}
$$

X'Y' Konfiguration $B_{2 g}$ Mode:

$$
\begin{gathered}
B_{2 g}=\left(\begin{array}{lll}
1 & 0 & 0
\end{array}\right) \cdot\left(\begin{array}{lll}
0 & 0 & e \\
0 & 0 & 0 \\
e & 0 & 0
\end{array}\right) \cdot\left(\begin{array}{c}
0 \\
-\frac{1}{\sqrt{2}} \\
\frac{1}{\sqrt{2}}
\end{array}\right)=\frac{1}{\sqrt{2}} e \\
B_{2 g}=\left(\begin{array}{lll}
0 & \frac{1}{\sqrt{2}} & -\frac{1}{\sqrt{2}}
\end{array}\right) \cdot\left(\begin{array}{lll}
0 & 0 & e \\
0 & 0 & 0 \\
e & 0 & 0
\end{array}\right) \cdot\left(\begin{array}{l}
1 \\
0 \\
0
\end{array}\right)=-\frac{1}{\sqrt{2} e} \\
B_{2 g}=\left(\begin{array}{lll}
\frac{1}{2} & \frac{2+\sqrt{2}}{4} & \frac{2-\sqrt{2}}{4}
\end{array}\right) \cdot\left(\begin{array}{lll}
0 & 0 & e \\
0 & 0 & 0 \\
e & 0 & 0
\end{array}\right) \cdot\left(\begin{array}{l}
\frac{-1}{2} \\
\frac{2-\sqrt{2}}{4} \\
\frac{2+\sqrt{2}}{4}
\end{array}\right)=\frac{\sqrt{2}}{4} d \\
B_{2 g}=\left(\begin{array}{lll}
-\frac{1}{2} & \frac{2+\sqrt{2}}{4} & \frac{2-\sqrt{2}}{4}
\end{array}\right) \cdot\left(\begin{array}{lll}
0 & 0 & e \\
0 & 0 & 0 \\
e & 0 & 0
\end{array}\right) \cdot\left(\begin{array}{c}
\frac{1}{2} \\
\frac{2-\sqrt{2}}{4} \\
\frac{2+\sqrt{2}}{4}
\end{array}\right)=\frac{\sqrt{2}}{4} d \\
B_{2 g}=\left(\begin{array}{lll}
-\frac{1}{2} & \frac{2-\sqrt{2}}{4} & \frac{2+\sqrt{2}}{4}
\end{array}\right) \cdot\left(\begin{array}{lll}
0 & 0 & e \\
0 & 0 & 0 \\
e & 0 & 0
\end{array}\right) \cdot\left(\begin{array}{c}
\frac{-1}{2} \\
\frac{-2-\sqrt{2}}{4} \\
\frac{-2+\sqrt{2}}{4}
\end{array}\right)=\frac{\sqrt{2}}{4} d \\
B_{2 g}=\left(\begin{array}{llll}
\frac{1}{2} & \frac{2-\sqrt{2}}{4} & \frac{2+\sqrt{2}}{4}
\end{array}\right) \cdot\left(\begin{array}{lll}
0 & 0 & e \\
0 & 0 & 0 \\
e & 0 & 0
\end{array}\right) \cdot\left(\begin{array}{c}
\frac{1}{2} \\
\frac{-2-\sqrt{2}}{4} \\
\frac{-2+\sqrt{2}}{4}
\end{array}\right)=\frac{\sqrt{2}}{4} d \\
140
\end{gathered}
$$


XX Konfiguration $B_{3 g}$ Mode:

$$
\begin{gathered}
B_{3 g}=\left(\begin{array}{lll}
\frac{1}{\sqrt{2}} & \frac{1}{2} & -\frac{1}{2}
\end{array}\right) \cdot\left(\begin{array}{lll}
0 & 0 & 0 \\
0 & 0 & f \\
0 & f & 0
\end{array}\right) \cdot\left(\begin{array}{c}
\frac{1}{\sqrt{2}} \\
\frac{1}{2} \\
-\frac{1}{2}
\end{array}\right)=\frac{1}{2} f \\
B_{3 g}=\left(\begin{array}{lll}
-\frac{1}{\sqrt{2}} & \frac{1}{2} & -\frac{1}{2}
\end{array}\right) \cdot\left(\begin{array}{lll}
0 & 0 & 0 \\
0 & 0 & f \\
0 & f & 0
\end{array}\right) \cdot\left(\begin{array}{c}
-\frac{1}{\sqrt{2}} \\
\frac{1}{2} \\
-\frac{1}{2}
\end{array}\right)=\frac{1}{2} f \\
B_{3 g}=\left(\begin{array}{lll}
\frac{1}{\sqrt{2}} & \frac{1}{2} & -\frac{1}{2}
\end{array}\right) \cdot\left(\begin{array}{lll}
0 & 0 & 0 \\
0 & 0 & f \\
0 & f & 0
\end{array}\right) \cdot\left(\begin{array}{c}
\frac{1}{\sqrt{2}} \\
\frac{1}{2} \\
-\frac{1}{2}
\end{array}\right)=\frac{1}{2} f \\
B_{3 g}=\left(\begin{array}{lll}
-\frac{1}{\sqrt{2}} & \frac{1}{2} & -\frac{1}{2}
\end{array}\right) \cdot\left(\begin{array}{lll}
0 & 0 & 0 \\
0 & 0 & f \\
0 & f & 0
\end{array}\right) \cdot\left(\begin{array}{c}
-\frac{1}{\sqrt{2}} \\
\frac{1}{2} \\
-\frac{1}{2}
\end{array}\right)=\frac{1}{2} f \\
B_{3 g}=\left(\begin{array}{lll}
0 & \frac{1}{\sqrt{2}} & \frac{1}{\sqrt{2}}
\end{array}\right) \cdot\left(\begin{array}{lll}
0 & 0 & 0 \\
0 & 0 & f \\
0 & f & 0
\end{array}\right) \cdot\left(\begin{array}{c}
0 \\
\frac{1}{\sqrt{2}} \\
\frac{1}{\sqrt{2}}
\end{array}\right)=f \\
B_{3 g}=\left(\begin{array}{lll}
0 & \frac{1}{\sqrt{2}} & \frac{1}{\sqrt{2}}
\end{array}\right) \cdot\left(\begin{array}{lll}
0 & 0 & 0 \\
0 & 0 & f \\
0 & f & 0
\end{array}\right) \cdot\left(\begin{array}{c}
0 \\
\frac{1}{\sqrt{2}} \\
\frac{1}{\sqrt{2}}
\end{array}\right)=f
\end{gathered}
$$

XY Konfiguration $B_{3 g}$ Mode:

$$
\begin{gathered}
B_{3 g}=\left(\begin{array}{lll}
\frac{1}{\sqrt{2}} & \frac{1}{2} & -\frac{1}{2}
\end{array}\right) \cdot\left(\begin{array}{lll}
0 & 0 & 0 \\
0 & 0 & f \\
0 & f & 0
\end{array}\right) \cdot\left(\begin{array}{c}
\frac{1}{\sqrt{2}} \\
-\frac{1}{2} \\
\frac{1}{2}
\end{array}\right)=\frac{1}{2} f \\
B_{3 g}=\left(\begin{array}{lll}
-\frac{1}{\sqrt{2}} & \frac{1}{2} & -\frac{1}{2}
\end{array}\right) \cdot\left(\begin{array}{lll}
0 & 0 & 0 \\
0 & 0 & f \\
0 & f & 0
\end{array}\right) \cdot\left(\begin{array}{c}
\frac{1}{\sqrt{2}} \\
\frac{1}{2} \\
-\frac{1}{2}
\end{array}\right)=\frac{1}{2} f \\
B_{3 g}=\left(\begin{array}{lll}
\frac{1}{\sqrt{2}} & \frac{1}{2} & -\frac{1}{2}
\end{array}\right) \cdot\left(\begin{array}{lll}
0 & 0 & 0 \\
0 & 0 & f \\
0 & f & 0
\end{array}\right) \cdot\left(\begin{array}{c}
0 \\
\frac{1}{\sqrt{2}} \\
\frac{1}{\sqrt{2}}
\end{array}\right)=0 \\
B_{3 g}=\left(\begin{array}{lll}
-\frac{1}{\sqrt{2}} & \frac{1}{2} & -\frac{1}{2}
\end{array}\right) \cdot\left(\begin{array}{lll}
0 & 0 & 0 \\
0 & 0 & f \\
0 & f & 0
\end{array}\right) \cdot\left(\begin{array}{c}
0 \\
\frac{1}{\sqrt{2}} \\
\frac{1}{\sqrt{2}}
\end{array}\right)=0
\end{gathered}
$$




$$
\begin{gathered}
B_{3 g}=\left(\begin{array}{lll}
0 & \frac{1}{\sqrt{2}} & \frac{1}{\sqrt{2}}
\end{array}\right) \cdot\left(\begin{array}{lll}
0 & 0 & 0 \\
0 & 0 & f \\
0 & f & 0
\end{array}\right) \cdot\left(\begin{array}{c}
-\frac{1}{\sqrt{2}} \\
-\frac{1}{2} \\
\frac{1}{2}
\end{array}\right)=0 \\
B_{3 g}=\left(\begin{array}{lll}
0 & \frac{1}{\sqrt{2}} & \frac{1}{\sqrt{2}}
\end{array}\right) \cdot\left(\begin{array}{lll}
0 & 0 & 0 \\
0 & 0 & f \\
0 & f & 0
\end{array}\right) \cdot\left(\begin{array}{c}
\frac{1}{\sqrt{2}} \\
-\frac{1}{2} \\
\frac{1}{2}
\end{array}\right)=0
\end{gathered}
$$

X'X' Konfiguration $B_{3 g}$ Mode:

$$
\begin{gathered}
B_{3 g}=\left(\begin{array}{lll}
1 & 0 & 0
\end{array}\right) \cdot\left(\begin{array}{lll}
0 & 0 & 0 \\
0 & 0 & f \\
0 & f & 0
\end{array}\right) \cdot\left(\begin{array}{l}
1 \\
0 \\
0
\end{array}\right)=0 \\
B_{3 g}=\left(\begin{array}{lll}
0 & \frac{1}{\sqrt{2}} & -\frac{1}{\sqrt{2}}
\end{array}\right) \cdot\left(\begin{array}{lll}
0 & 0 & 0 \\
0 & 0 & f \\
0 & f & 0
\end{array}\right) \cdot\left(\begin{array}{c}
0 \\
\frac{1}{\sqrt{2}} \\
-\frac{1}{\sqrt{2}}
\end{array}\right)=f \\
B_{3 g}=\left(\begin{array}{lll}
\frac{1}{2} & \frac{2+\sqrt{2}}{4} & \frac{2-\sqrt{2}}{4}
\end{array}\right) \cdot\left(\begin{array}{lll}
0 & 0 & 0 \\
0 & 0 & f \\
0 & f & 0
\end{array}\right) \cdot\left(\begin{array}{c}
\frac{1}{2} \\
\frac{2+\sqrt{2}}{4} \\
\frac{2-\sqrt{2}}{4}
\end{array}\right)=\frac{3}{8} f \\
B_{3 g}=\left(\begin{array}{lll}
-\frac{1}{2} & \frac{2+\sqrt{2}}{4} & \frac{2-\sqrt{2}}{4}
\end{array}\right) \cdot\left(\begin{array}{lll}
0 & 0 & 0 \\
0 & 0 & f \\
0 & f & 0
\end{array}\right) \cdot\left(\begin{array}{c}
-\frac{1}{2} \\
\frac{2+\sqrt{2}}{4} \\
\frac{2-\sqrt{2}}{4}
\end{array}\right)=\frac{3}{8} f \\
B_{3 g}=\left(\begin{array}{lll}
-\frac{1}{2} & \frac{2-\sqrt{2}}{4} & \frac{2+\sqrt{2}}{4}
\end{array}\right) \cdot\left(\begin{array}{lll}
0 & 0 & 0 \\
0 & 0 & f \\
0 & f & 0
\end{array}\right) \cdot\left(\begin{array}{c}
-\frac{1}{2} \\
\frac{2-\sqrt{2}}{4} \\
\frac{2+\sqrt{2}}{4}
\end{array}\right)=\frac{3}{8} f \\
B_{3 g}=\left(\begin{array}{lll}
\frac{1}{2} & \frac{2-\sqrt{2}}{4} & \frac{2+\sqrt{2}}{4}
\end{array}\right) \cdot\left(\begin{array}{lll}
0 & 0 & 0 \\
0 & 0 & f \\
0 & f & 0
\end{array}\right) \cdot\left(\begin{array}{c}
\frac{1}{2} \\
\frac{2-\sqrt{2}}{4} \\
\frac{2+\sqrt{2}}{4}
\end{array}\right)=\frac{3}{8} f
\end{gathered}
$$

X'Y' Konfiguration $B_{3 g}$ Mode:

$$
\begin{gathered}
B_{3 g}=\left(\begin{array}{lll}
1 & 0 & 0
\end{array}\right) \cdot\left(\begin{array}{lll}
0 & 0 & 0 \\
0 & 0 & f \\
0 & f & 0
\end{array}\right) \cdot\left(\begin{array}{c}
0 \\
-\frac{1}{\sqrt{2}} \\
\frac{1}{\sqrt{2}}
\end{array}\right)=0 \\
B_{3 g}=\left(\begin{array}{lll}
0 & \frac{1}{\sqrt{2}} & -\frac{1}{\sqrt{2}}
\end{array}\right) \cdot\left(\begin{array}{lll}
0 & 0 & 0 \\
0 & 0 & f \\
0 & f & 0
\end{array}\right) \cdot\left(\begin{array}{l}
1 \\
0 \\
0
\end{array}\right)=0
\end{gathered}
$$




$$
\begin{aligned}
& B_{3 g}=\left(\begin{array}{lll}
\frac{1}{2} & \frac{2+\sqrt{2}}{4} & \frac{2-\sqrt{2}}{4}
\end{array}\right) \cdot\left(\begin{array}{lll}
0 & 0 & 0 \\
0 & 0 & f \\
0 & f & 0
\end{array}\right) \cdot\left(\begin{array}{c}
\frac{-1}{2} \\
\frac{2-\sqrt{2}}{4} \\
\frac{2+\sqrt{2}}{4}
\end{array}\right)=\left(\frac{2+\sqrt{2}}{4}\right)^{2} f+\left(\frac{2-\sqrt{2}}{4}\right)^{2} f \\
& B_{3 g}=\left(\begin{array}{lll}
-\frac{1}{2} & \frac{2+\sqrt{2}}{4} & \frac{2-\sqrt{2}}{4}
\end{array}\right) \cdot\left(\begin{array}{lll}
0 & 0 & 0 \\
0 & 0 & f \\
0 & f & 0
\end{array}\right) \cdot\left(\begin{array}{c}
\frac{1}{2} \\
\frac{2-\sqrt{2}}{4} \\
\frac{2+\sqrt{2}}{4}
\end{array}\right)=\left(\frac{2+\sqrt{2}}{4}\right)^{2} f+\left(\frac{2-\sqrt{2}}{4}\right)^{2} f \\
& B_{3 g}=\left(\begin{array}{lll}
-\frac{1}{2} & \frac{2-\sqrt{2}}{4} & \frac{2+\sqrt{2}}{4}
\end{array}\right) \cdot\left(\begin{array}{lll}
0 & 0 & 0 \\
0 & 0 & f \\
0 & f & 0
\end{array}\right) \cdot\left(\begin{array}{c}
\frac{-\frac{1}{2}}{4} \\
\frac{-2+\sqrt{2}}{4}
\end{array}\right)=\left(\frac{2+\sqrt{2}}{4}\right)^{2} f+\left(\frac{2-\sqrt{2}}{4}\right)^{2} f \\
& B_{3 g}=\left(\begin{array}{lll}
\frac{1}{2} & \frac{2-\sqrt{2}}{4} & \frac{2+\sqrt{2}}{4}
\end{array}\right) \cdot\left(\begin{array}{lll}
0 & 0 & 0 \\
0 & 0 & f \\
0 & f & 0
\end{array}\right) \cdot\left(\begin{array}{c}
\frac{1}{2} \\
\frac{-2-\sqrt{2}}{4} \\
\frac{-2+\sqrt{2}}{4}
\end{array}\right)=\left(\frac{2+\sqrt{2}}{4}\right)^{2} f+\left(\frac{2-\sqrt{2}}{4}\right)^{2} f
\end{aligned}
$$

Alle diese Elemente müssen noch quadriert und mit dem Faktor 1/6 gewichtet werden. Das Ergebnis der mittleren Intensität ergibt sich dann zu:

\begin{tabular}{|c|c|}
\hline & $A_{g}$ \\
\hline $\mathrm{XX}$ & $\frac{1}{6}\left[\frac{1}{4}(2 a+c+b)^{2}+\frac{1}{2}(b+c)^{2}\right]$ \\
\hline $\mathrm{XY}$ & $\frac{1}{6}\left[\frac{1}{8}(2 a-b-c)^{2}+\frac{1}{2}(b-c)^{2}\right]$ \\
\hline $\mathrm{X}^{\prime} \mathrm{X}^{\prime}$ & $\frac{1}{6}\left[a^{2}+\frac{1}{4}(b+c)^{2}+\frac{1}{32}(2 a+(3+\sqrt{2}) b+(3-\sqrt{2}) c)^{2}+\frac{1}{32}(2 a+(3-\sqrt{2}) b+(3+\sqrt{2}) c)^{2}\right]$ \\
\hline $\mathrm{X}^{\prime} Y^{\prime}$ & $\frac{1}{6}\left[\frac{1}{16}(2 a-b-c)^{2}\right]$ \\
\hline
\end{tabular}

\begin{tabular}{|c|c|c|c|c|}
\hline Mode & XX & XY & $X^{\prime} X^{\prime}$ & $X^{\prime} Y^{\prime}$ \\
\hline$B_{1 g}$ & $\frac{2}{6} d^{2}$ & $\frac{1}{6} d^{2}$ & $\frac{1}{6}\left(\frac{3}{4} d^{2}\right)$ & $\frac{1}{6}\left(d^{2}+\frac{1}{4} d^{2}\right)$ \\
\hline$B_{2 g}$ & $\frac{2}{6} e^{2}$ & $\frac{1}{6} e^{2}$ & $\frac{1}{6}\left(e^{2}+\frac{1}{4} e^{2}\right)$ & $\frac{1}{6}\left(2 \frac{3}{4} e^{2}\right)$ \\
\hline$B_{3 g}$ & $\frac{1}{6}\left(\frac{3}{4} f^{2}+2 f^{2}\right)$ & $\frac{1}{12} f^{2}$ & $\frac{1}{6}\left(\frac{1}{4} f^{2}+f^{2}\right)$ & $\frac{1}{6}\left(4 \frac{35}{64} f^{2}\right)$ \\
\hline
\end{tabular}

Damit ergeben sich die Intensitätsverhältnisse für die (011)-Orientierung $\mathrm{zu}:$ 


\begin{tabular}{|c|c|c|c|c|}
\hline Mode & XX & XY & $\mathrm{X}^{\prime} \mathrm{X}^{\prime}$ & $\mathrm{X}^{\prime} \mathrm{Y}^{\prime}$ \\
\hline$B_{1 g}$ & 2 & 1 & 1,5 & 1,5 \\
\hline$B_{2 g}$ & 2 & 1 & 1,5 & 1,5 \\
\hline$B_{3 g}$ & 2,75 & 0,5 & 1,25 & 2,1875 \\
\hline$A_{g}(a=b=c)$ & 6 & 0 & 6 & 0 \\
\hline$A_{g}(a=c \gg b)$ & 2,75 & 0,625 & 2,935 & 0,0625 \\
\hline$A_{g}(a=c \ll b)$ & 0,75 & 0,625 & 0,936 & 0,0625 \\
\hline
\end{tabular}

Tabelle 12 - Intensitätsverhältnisse für die Proben mit (011)-

Orientierung

Die Berechnung der Intensitäten für die $P 2_{1} / m$-Struktur erfolgt equivalent. Es können hier die Zwillinge für die [001]-Richtung verwendet werden.

XX Konfiguration $A_{g}$ Mode:

$$
\begin{gathered}
A_{g}=\left(\begin{array}{lll}
\frac{1}{\sqrt{2}} & 0 & -\frac{1}{\sqrt{2}}
\end{array}\right) \cdot\left(\begin{array}{lll}
a & d & 0 \\
d & b & 0 \\
0 & 0 & c
\end{array}\right) \cdot\left(\begin{array}{c}
\frac{1}{\sqrt{2}} \\
0 \\
-\frac{1}{\sqrt{2}}
\end{array}\right)=\frac{1}{2}(a+c) \\
A_{g}=\left(\begin{array}{lll}
-\frac{1}{\sqrt{2}} & 0 & -\frac{1}{\sqrt{2}}
\end{array}\right) \cdot\left(\begin{array}{lll}
a & d & 0 \\
d & b & 0 \\
0 & 0 & c
\end{array}\right) \cdot\left(\begin{array}{c}
-\frac{1}{\sqrt{2}} \\
0 \\
-\frac{1}{\sqrt{2}}
\end{array}\right)=\frac{1}{2}(a+c) \\
A_{g}=\left(\begin{array}{lll}
\frac{1}{\sqrt{2}} & 0 & -\frac{1}{\sqrt{2}}
\end{array}\right) \cdot\left(\begin{array}{lll}
a & d & 0 \\
d & b & 0 \\
0 & 0 & c
\end{array}\right) \cdot\left(\begin{array}{c}
\frac{1}{\sqrt{2}} \\
0 \\
-\frac{1}{\sqrt{2}}
\end{array}\right)=\frac{1}{2}(a+c) \\
A_{g}=\left(\begin{array}{lll}
-\frac{1}{\sqrt{2}} & 0 & -\frac{1}{\sqrt{2}}
\end{array}\right) \cdot\left(\begin{array}{lll}
a & d & 0 \\
d & b & 0 \\
0 & 0 & c
\end{array}\right) \cdot\left(\begin{array}{c}
-\frac{1}{\sqrt{2}} \\
0 \\
-\frac{1}{\sqrt{2}}
\end{array}\right)=\frac{1}{2}(a+c) \\
A_{g}=\left(\begin{array}{lll}
0 & 1 & 0
\end{array}\right) \cdot\left(\begin{array}{lll}
a & d & 0 \\
d & b & 0 \\
0 & 0 & c
\end{array}\right) \cdot\left(\begin{array}{c}
0 \\
1 \\
0
\end{array}\right)=b \\
A_{g}=\left(\begin{array}{lll}
0 & 1 & 0
\end{array}\right) \cdot\left(\begin{array}{lll}
a & d & 0 \\
d & b & 0 \\
0 & 0 & c
\end{array}\right) \cdot\left(\begin{array}{l}
0 \\
1 \\
0
\end{array}\right)=b
\end{gathered}
$$

XY Konfiguration $A_{g}$ Mode:

$$
A_{g}=\left(\begin{array}{lll}
\frac{1}{\sqrt{2}} & 0 & -\frac{1}{\sqrt{2}}
\end{array}\right) \cdot\left(\begin{array}{lll}
a & d & 0 \\
d & b & 0 \\
0 & 0 & c
\end{array}\right) \cdot\left(\begin{array}{c}
\frac{1}{\sqrt{2}} \\
0 \\
\frac{1}{\sqrt{2}}
\end{array}\right)=\frac{1}{2}(a-c)
$$




$$
\begin{gathered}
A_{g}=\left(\begin{array}{lll}
-\frac{1}{\sqrt{2}} & 0 & -\frac{1}{\sqrt{2}}
\end{array}\right) \cdot\left(\begin{array}{lll}
a & d & 0 \\
d & b & 0 \\
0 & 0 & c
\end{array}\right) \cdot\left(\begin{array}{c}
\frac{1}{\sqrt{2}} \\
0 \\
-\frac{1}{\sqrt{2}}
\end{array}\right)=\frac{1}{2}(a-c) \\
A_{g}=\left(\begin{array}{lll}
\frac{1}{\sqrt{2}} & 0 & -\frac{1}{\sqrt{2}}
\end{array}\right) \cdot\left(\begin{array}{lll}
a & d & 0 \\
d & b & 0 \\
0 & 0 & c
\end{array}\right) \cdot\left(\begin{array}{l}
0 \\
1 \\
0
\end{array}\right)=\frac{1}{\sqrt{2}} \cdot d \\
A_{g}=\left(\begin{array}{lll}
-\frac{1}{\sqrt{2}} & 0 & -\frac{1}{\sqrt{2}}
\end{array}\right) \cdot\left(\begin{array}{lll}
a & d & 0 \\
d & b & 0 \\
0 & 0 & c
\end{array}\right) \cdot\left(\begin{array}{l}
0 \\
1 \\
0
\end{array}\right)=\frac{1}{\sqrt{2}} \cdot d \\
A_{g}=\left(\begin{array}{lll}
0 & 1 & 0
\end{array}\right) \cdot\left(\begin{array}{lll}
a & d & 0 \\
d & b & 0 \\
0 & 0 & c
\end{array}\right) \cdot\left(\begin{array}{c}
-\frac{1}{\sqrt{2}} \\
0 \\
\frac{1}{\sqrt{2}}
\end{array}\right)=\frac{1}{\sqrt{2}} \cdot d \\
A_{g}=\left(\begin{array}{lll}
0 & 1 & 0
\end{array}\right) \cdot\left(\begin{array}{lll}
a & d & 0 \\
d & b & 0 \\
0 & 0 & c
\end{array}\right) \cdot\left(\begin{array}{c}
\frac{1}{\sqrt{2}} \\
0 \\
\frac{1}{\sqrt{2}}
\end{array}\right)=\frac{1}{\sqrt{2}} \cdot d
\end{gathered}
$$

X'X' Konfiguration $A_{g}$ Mode:

$$
\begin{gathered}
A_{g}=\left(\begin{array}{lll}
1 & 0 & 0
\end{array}\right) \cdot\left(\begin{array}{lll}
a & d & 0 \\
d & b & 0 \\
0 & 0 & c
\end{array}\right) \cdot\left(\begin{array}{c}
1 \\
0 \\
0
\end{array}\right)=a \\
A_{g}=\left(\begin{array}{lll}
0 & 0 & -1
\end{array}\right) \cdot\left(\begin{array}{lll}
a & d & 0 \\
d & b & 0 \\
0 & 0 & c
\end{array}\right) \cdot\left(\begin{array}{c}
0 \\
0 \\
-1
\end{array}\right)=c \\
A_{g}=\left(\begin{array}{lll}
\frac{1}{2} & \frac{1}{\sqrt{2}} & -\frac{1}{2}
\end{array}\right) \cdot\left(\begin{array}{lll}
a & d & 0 \\
d & b & 0 \\
0 & 0 & c
\end{array}\right) \cdot\left(\begin{array}{c}
\frac{1}{2} \\
\frac{1}{\sqrt{2}} \\
-\frac{1}{2}
\end{array}\right)=\frac{1}{4}(a+2 b+c+2 \sqrt{2} d) \\
A_{g}=\left(\begin{array}{lll}
-\frac{1}{2} & \frac{1}{\sqrt{2}} & -\frac{1}{2}
\end{array}\right) \cdot\left(\begin{array}{lll}
a & d & 0 \\
d & b & 0 \\
0 & 0 & c
\end{array}\right) \cdot\left(\begin{array}{c}
-\frac{1}{2} \\
\frac{1}{\sqrt{2}} \\
-\frac{1}{2}
\end{array}\right)=\frac{1}{4}(a+2 b+c+2 \sqrt{2} d) \\
A_{g}=\left(\begin{array}{lll}
-\frac{1}{2} & \frac{1}{\sqrt{2}} & \frac{1}{2}
\end{array}\right) \cdot\left(\begin{array}{lll}
a & d & 0 \\
d & b & 0 \\
0 & 0 & c
\end{array}\right) \cdot\left(\begin{array}{c}
-\frac{1}{2} \\
\frac{1}{\sqrt{2}} \\
\frac{1}{2}
\end{array}\right)=\frac{1}{4}(a+2 b+c+2 \sqrt{2} d) \\
A_{g}=\left(\begin{array}{lll}
\frac{1}{2} & \frac{1}{\sqrt{2}} & \frac{1}{2}
\end{array}\right) \cdot\left(\begin{array}{lll}
a & d & 0 \\
d & b & 0 \\
0 & 0 & c
\end{array}\right) \cdot\left(\begin{array}{c}
\frac{1}{2} \\
\frac{1}{\sqrt{2}} \\
\frac{1}{2}
\end{array}\right)=\frac{1}{4}(a+2 b+c+2 \sqrt{2} d)
\end{gathered}
$$


X'Y' Konfiguration $A_{g}$ Mode:

$$
\begin{gathered}
A_{g}=\left(\begin{array}{lll}
1 & 0 & 0
\end{array}\right) \cdot\left(\begin{array}{lll}
a & d & 0 \\
d & b & 0 \\
0 & 0 & c
\end{array}\right) \cdot\left(\begin{array}{l}
0 \\
0 \\
1
\end{array}\right)=0 \\
A_{g}=\left(\begin{array}{lll}
0 & 0 & -1
\end{array}\right) \cdot\left(\begin{array}{lll}
a & d & 0 \\
d & b & 0 \\
0 & 0 & c
\end{array}\right) \cdot\left(\begin{array}{l}
1 \\
0 \\
0
\end{array}\right)=0 \\
A_{g}=\left(\begin{array}{lll}
\frac{1}{2} & \frac{1}{\sqrt{2}} & -\frac{1}{2}
\end{array}\right) \cdot\left(\begin{array}{lll}
a & d & 0 \\
d & b & 0 \\
0 & 0 & c
\end{array}\right) \cdot\left(\begin{array}{c}
-\frac{1}{2} \\
\frac{1}{\sqrt{2}} \\
\frac{1}{2}
\end{array}\right)=\frac{1}{4}(-a+2 b-c) \\
A_{g}=\left(\begin{array}{lll}
-\frac{1}{2} & \frac{1}{\sqrt{2}} & -\frac{1}{2}
\end{array}\right) \cdot\left(\begin{array}{lll}
a & d & 0 \\
d & b & 0 \\
0 & 0 & c
\end{array}\right) \cdot\left(\begin{array}{c}
\frac{1}{2} \\
\frac{1}{\sqrt{2}} \\
\frac{1}{2}
\end{array}\right)=\frac{1}{4}(-a+2 b-c) \\
A_{g}=\left(\begin{array}{lll}
-\frac{1}{2} & \frac{1}{\sqrt{2}} & \frac{1}{2}
\end{array}\right) \cdot\left(\begin{array}{lll}
a & d & 0 \\
d & b & 0 \\
0 & 0 & c
\end{array}\right) \cdot\left(\begin{array}{c}
-\frac{1}{2} \\
-\frac{1}{\sqrt{2}} \\
\frac{1}{2}
\end{array}\right)=\frac{1}{4}(-a+2 b-c) \\
A_{g}=\left(\begin{array}{lll}
\frac{1}{2} & \frac{1}{\sqrt{2}} & \frac{1}{2}
\end{array}\right) \cdot\left(\begin{array}{lll}
a & d & 0 \\
d & b & 0 \\
0 & 0 & c
\end{array}\right) \cdot\left(\begin{array}{c}
\frac{1}{2} \\
-\frac{1}{\sqrt{2}} \\
\frac{1}{2}
\end{array}\right)=\frac{1}{4}(-a+2 b-c)
\end{gathered}
$$

XX Konfiguration $B_{g}$ Mode:

$$
\begin{gathered}
B_{g}=\left(\begin{array}{lll}
\frac{1}{\sqrt{2}} & 0 & -\frac{1}{\sqrt{2}}
\end{array}\right) \cdot\left(\begin{array}{lll}
0 & 0 & e \\
0 & 0 & f \\
e & f & 0
\end{array}\right) \cdot\left(\begin{array}{c}
\frac{1}{\sqrt{2}} \\
0 \\
-\frac{1}{\sqrt{2}}
\end{array}\right)=e \\
B_{g}=\left(\begin{array}{lll}
-\frac{1}{\sqrt{2}} & 0 & -\frac{1}{\sqrt{2}}
\end{array}\right) \cdot\left(\begin{array}{lll}
0 & 0 & e \\
0 & 0 & f \\
e & f & 0
\end{array}\right) \cdot\left(\begin{array}{c}
-\frac{1}{\sqrt{2}} \\
0 \\
-\frac{1}{\sqrt{2}}
\end{array}\right)=e \\
B_{g}=\left(\begin{array}{lll}
\frac{1}{\sqrt{2}} & 0 & -\frac{1}{\sqrt{2}}
\end{array}\right) \cdot\left(\begin{array}{lll}
0 & 0 & e \\
0 & 0 & f \\
e & f & 0
\end{array}\right) \cdot\left(\begin{array}{c}
\frac{1}{\sqrt{2}} \\
0 \\
-\frac{1}{\sqrt{2}}
\end{array}\right)=e \\
B_{g}=\left(\begin{array}{lll}
-\frac{1}{\sqrt{2}} & 0 & -\frac{1}{\sqrt{2}}
\end{array}\right) \cdot\left(\begin{array}{lll}
0 & 0 & e \\
0 & 0 & f \\
e & f & 0
\end{array}\right) \cdot\left(\begin{array}{c}
-\frac{1}{\sqrt{2}} \\
0 \\
-\frac{1}{\sqrt{2}}
\end{array}\right)=e
\end{gathered}
$$




$$
\begin{aligned}
& B_{g}=\left(\begin{array}{lll}
0 & 1 & 0
\end{array}\right) \cdot\left(\begin{array}{lll}
0 & 0 & e \\
0 & 0 & f \\
e & f & 0
\end{array}\right) \cdot\left(\begin{array}{l}
0 \\
1 \\
0
\end{array}\right)=0 \\
& B_{g}=\left(\begin{array}{lll}
0 & 1 & 0
\end{array}\right) \cdot\left(\begin{array}{lll}
0 & 0 & e \\
0 & 0 & f \\
e & f & 0
\end{array}\right) \cdot\left(\begin{array}{l}
0 \\
1 \\
0
\end{array}\right)=0
\end{aligned}
$$

XY Konfiguration $B_{g}$ Mode:

$$
\begin{gathered}
B_{g}=\left(\begin{array}{lll}
\frac{1}{\sqrt{2}} & 0 & -\frac{1}{\sqrt{2}}
\end{array}\right) \cdot\left(\begin{array}{lll}
0 & 0 & e \\
0 & 0 & f \\
e & f & 0
\end{array}\right) \cdot\left(\begin{array}{c}
\frac{1}{\sqrt{2}} \\
0 \\
\frac{1}{\sqrt{2}}
\end{array}\right)=0 \\
B_{g}=\left(\begin{array}{lll}
-\frac{1}{\sqrt{2}} & 0 & -\frac{1}{\sqrt{2}}
\end{array}\right) \cdot\left(\begin{array}{lll}
0 & 0 & e \\
0 & 0 & f \\
e & f & 0
\end{array}\right) \cdot\left(\begin{array}{c}
\frac{1}{\sqrt{2}} \\
0 \\
-\frac{1}{\sqrt{2}}
\end{array}\right)=0 \\
B_{g}=\left(\begin{array}{lll}
\frac{1}{\sqrt{2}} & 0 & -\frac{1}{\sqrt{2}}
\end{array}\right) \cdot\left(\begin{array}{lll}
0 & 0 & e \\
0 & 0 & f \\
e & f & 0
\end{array}\right) \cdot\left(\begin{array}{l}
0 \\
1 \\
0
\end{array}\right)=\frac{1}{\sqrt{2}} \cdot f \\
B_{g}=\left(\begin{array}{lll}
-\frac{1}{\sqrt{2}} & 0 & -\frac{1}{\sqrt{2}}
\end{array}\right) \cdot\left(\begin{array}{lll}
0 & 0 & e \\
0 & 0 & f \\
e & f & 0
\end{array}\right) \cdot\left(\begin{array}{l}
0 \\
1 \\
0
\end{array}\right)=\frac{1}{\sqrt{2}} \cdot f \\
B_{g}=\left(\begin{array}{lll}
0 & 1 & 0
\end{array}\right) \cdot\left(\begin{array}{lll}
0 & 0 & e \\
0 & 0 & f \\
e & f & 0
\end{array}\right) \cdot\left(\begin{array}{c}
-\frac{1}{\sqrt{2}} \\
0 \\
\frac{1}{\sqrt{2}}
\end{array}\right)=\frac{1}{\sqrt{2}} \cdot f \\
B_{g}=\left(\begin{array}{lll}
0 & 1 & 0
\end{array}\right) \cdot\left(\begin{array}{lll}
0 & 0 & e \\
0 & 0 & f \\
e & f & 0
\end{array}\right) \cdot\left(\begin{array}{c}
\frac{1}{\sqrt{2}} \\
0 \\
\frac{1}{\sqrt{2}}
\end{array}\right)=\frac{1}{\sqrt{2}} \cdot f
\end{gathered}
$$

X'X' Konfiguration $B_{g}$ Mode:

$$
\begin{gathered}
B_{g}=\left(\begin{array}{lll}
1 & 0 & 0
\end{array}\right) \cdot\left(\begin{array}{ccc}
0 & 0 & e \\
0 & 0 & f \\
e & f & 0
\end{array}\right) \cdot\left(\begin{array}{c}
1 \\
0 \\
0
\end{array}\right)=0 \\
B_{g}=\left(\begin{array}{lll}
0 & 0 & -1
\end{array}\right) \cdot\left(\begin{array}{lll}
0 & 0 & e \\
0 & 0 & f \\
e & f & 0
\end{array}\right) \cdot\left(\begin{array}{c}
0 \\
0 \\
-1
\end{array}\right)=0
\end{gathered}
$$




$$
\begin{gathered}
B_{g}=\left(\begin{array}{lll}
\frac{1}{2} & \frac{1}{\sqrt{2}} & -\frac{1}{2}
\end{array}\right) \cdot\left(\begin{array}{lll}
0 & 0 & e \\
0 & 0 & f \\
e & f & 0
\end{array}\right) \cdot\left(\begin{array}{c}
\frac{1}{2} \\
\frac{1}{\sqrt{2}} \\
-\frac{1}{2}
\end{array}\right)=\frac{1}{2} \cdot e+\frac{1}{\sqrt{2}} \cdot f \\
B_{g}=\left(\begin{array}{lll}
-\frac{1}{2} & \frac{1}{\sqrt{2}} & -\frac{1}{2}
\end{array}\right) \cdot\left(\begin{array}{lll}
0 & 0 & e \\
0 & 0 & f \\
e & f & 0
\end{array}\right) \cdot\left(\begin{array}{c}
-\frac{1}{2} \\
\frac{1}{\sqrt{2}} \\
-\frac{1}{2}
\end{array}\right)=\frac{1}{2} \cdot e+\frac{1}{\sqrt{2}} \cdot f \\
B_{g}=\left(\begin{array}{lll}
-\frac{1}{2} & \frac{1}{\sqrt{2}} & \frac{1}{2}
\end{array}\right) \cdot\left(\begin{array}{lll}
0 & 0 & e \\
0 & 0 & f \\
e & f & 0
\end{array}\right) \cdot\left(\begin{array}{c}
-\frac{1}{2} \\
\frac{1}{\sqrt{2}} \\
\frac{1}{2}
\end{array}\right)=\frac{1}{2} \cdot e+\frac{1}{\sqrt{2}} \cdot f \\
B_{g}=\left(\begin{array}{lll}
\frac{1}{2} & \frac{1}{\sqrt{2}} & \frac{1}{2}
\end{array}\right) \cdot\left(\begin{array}{lll}
0 & 0 & e \\
0 & 0 & f \\
e & f & 0
\end{array}\right) \cdot\left(\begin{array}{c}
\frac{1}{2} \\
\frac{1}{\sqrt{2}} \\
\frac{1}{2}
\end{array}\right)=\frac{1}{2} \cdot e+\frac{1}{\sqrt{2}} \cdot f
\end{gathered}
$$

X'Y' Konfiguration $B_{g}$ Mode:

$$
\begin{gathered}
B_{g}=\left(\begin{array}{lll}
1 & 0 & 0
\end{array}\right) \cdot\left(\begin{array}{lll}
0 & 0 & e \\
0 & 0 & f \\
e & f & 0
\end{array}\right) \cdot\left(\begin{array}{l}
0 \\
0 \\
1
\end{array}\right)=e \\
B_{g}=\left(\begin{array}{lll}
0 & 0 & -1
\end{array}\right) \cdot\left(\begin{array}{lll}
0 & 0 & e \\
0 & 0 & f \\
e & f & 0
\end{array}\right) \cdot\left(\begin{array}{c}
1 \\
0 \\
0
\end{array}\right)=e \\
B_{g}=\left(\begin{array}{lll}
\frac{1}{2} & \frac{1}{\sqrt{2}} & -\frac{1}{2}
\end{array}\right) \cdot\left(\begin{array}{lll}
0 & 0 & e \\
0 & 0 & f \\
e & f & 0
\end{array}\right) \cdot\left(\begin{array}{c}
-\frac{1}{2} \\
\frac{1}{\sqrt{2}} \\
\frac{1}{2}
\end{array}\right)=\frac{1}{2} \cdot e \\
B_{g}=\left(\begin{array}{lll}
-\frac{1}{2} & \frac{1}{\sqrt{2}} & -\frac{1}{2}
\end{array}\right) \cdot\left(\begin{array}{lll}
0 & 0 & e \\
0 & 0 & f \\
e & f & 0
\end{array}\right) \cdot\left(\begin{array}{c}
\frac{1}{2} \\
\frac{1}{\sqrt{2}} \\
\frac{1}{2}
\end{array}\right)=\frac{1}{2} \cdot e \\
B_{g}=\left(\begin{array}{lll}
-\frac{1}{2} & \frac{1}{\sqrt{2}} & \frac{1}{2}
\end{array}\right) \cdot\left(\begin{array}{lll}
0 & 0 & e \\
0 & 0 & f \\
e & f & 0
\end{array}\right) \cdot\left(\begin{array}{c}
-\frac{1}{2} \\
-\frac{1}{\sqrt{2}} \\
\frac{1}{2}
\end{array}\right)=\frac{1}{2} \cdot e \\
B_{g}=\left(\begin{array}{lll}
\frac{1}{2} & \frac{1}{\sqrt{2}} & \frac{1}{2}
\end{array}\right) \cdot\left(\begin{array}{lll}
0 & 0 & e \\
0 & 0 & f \\
e & f & 0
\end{array}\right) \cdot\left(\begin{array}{c}
\frac{1}{2} \\
-\frac{1}{\sqrt{2}} \\
\frac{1}{2}
\end{array}\right)=\frac{1}{2} \cdot e
\end{gathered}
$$


Wie auch zuvor müssen alle diese Elemente noch quadriert und mit dem Faktor 1/6 gewichtet werden. Das Ergebnis der mittleren Intensitäten ergibt sich dann zu:

\begin{tabular}{|c|c|c|}
\hline Konf. & $A_{g}$ & $B_{g}$ \\
\hline XX & $\frac{1}{6}\left[(a+c)^{2}+2 b^{2}\right]$ & $\frac{2}{3} e^{2}$ \\
\hline XY & $\frac{1}{6}\left[\frac{1}{2}(a-c)^{2}+2 d^{2}\right]$ & $\frac{1}{3}\left(f^{2}\right)$ \\
\hline$X^{\prime} X^{\prime}$ & $\frac{1}{6}\left[\frac{1}{4}(a+2 b+c+2 \sqrt{2} d)^{2}+a^{2}+c^{2}\right]$ & $\frac{1}{6}\left(e^{2}+2 f^{2}\right)$ \\
\hline$X^{\prime} Y^{\prime}$ & $\frac{1}{24}(-a+2 b-c)^{2}$ & $\frac{1}{2}\left(e^{2}\right)$ \\
\hline \multicolumn{2}{|c|}{ Tabelle $\mathbf{1 3}-$ Intensitätsverhältnisse der P2 $2_{1} /$-Struktur }
\end{tabular}




\section{$9 \quad$ Danksagung}

Bei einer Doktorarbeit ist das Schwierige nicht das Anfangen sondern das Aufhören. Im Laufe meiner Arbeit habe ich viele Gesichter kommen und gehen sehen und viele meiner Kollegen sind zu innigen Freunden geworden. Ich möchte mich daher bei allen Menschen, die mir diese Arbeit erst ermöglicht haben, bedanken.

Zuallererst Vasily Moshnyaga ohne den ich die Manganate niemals kennengelernt hätte. Nach der erfolgreichen Diplomarbeit ermöglichte er mir, auch als Doktorand, weiterzuarbeiten und meine Kenntnisse weiter zu vertiefen. Meinen tiefempfundenen Dank für die tollen Jahre und die Möglichkeit in der MAD-Gruppe mitzuwirken. Außerdem großen Dank für die immer offene Tür und die Bereitschaft Probleme zu besprechen.

Konrad Samwer für die Aufnahme ins 1. Physikalische Institut und damit in den Kreis solcher Größen wie Lichtenberg und Pohl.

Vladimir Roddatis aus dem Institut für Materialphysik für die Hilfe bei den TEM-Messungen.

Boris Gorshunov und Elena Zhukova für die Hilfe bei den THz- und Infrarotmessungen.

Den drei Musketieren: (ja ich mochte diesen Namen immer lieber, denn er passt besser)

„Einer für alle, alle für Einen!“

Sebastian Hühn, der das Grillen zu einer Kunst erhob und mich oft genug mit klugen Fragen zu tieferer Einsicht brachte. Sowie für das Korrekturlesen meiner Arbeit

Markus Jungbauer, der immer mehr wollte als die Anderen vor Ihm und auf diese Weise auch die MAD-Gruppe mit sich zog. Auch hier ein Dank für das Korrekturlesen.

Markus Michelmann, der oft den Ruhepunkt darstellte und mit konstruktiver Kritik den Diskussionen Halt gab.

Sebastian Merten, für die Hilfe am Kryostaten für die temperaturabhängigen Ramanmessungen. Christoph Meier, für die interessanten Messungen am TERS. Wir hatten eine tolle Zeit zusammen und jeder von euch war immer bereit zu helfen. Das werde ich niemals vergessen.

Ein großes Dankeschön an alle Mitglieder der MAD-Gruppe: Kai Gehrke, Jon-Olaf Krisponeit, Christin Kalkert, Melanie Schneider, Camilo Balani, Victor Pfahl, Danny Schwarzbach, Christoph Meyer, Vitaly Bruchmann-Bamberg, Marius Keunicke, Eduard Unger und Alle die ich vergessen habe.

Außerdem danken möchte ich den restlichen Mitgliedern des Instituts sowie der Werkstatt für jede Hilfe und viel Kurzweil.

Einen besonderen Dank an Wenjuan Chen, die zwar erst im letzten Viertel 
der Arbeit zu mir stieß, aber mir den Eifer gab fertig zu schreiben um wieder nach China zu reisen. Außerdem einen Dank an meine Familie, die mich bei allen Entscheidungen unterstützt hat. 